\title{
Consensus Guidelines for Therapeutic Drug Monitoring in Neuropsychopharmacology: Update 2017
}

Authors

C. Hiemke1, ${ }^{1}$, N. Bergemann ${ }^{3}$, H. W. Clement ${ }^{4}$, A. Conca ${ }^{5}$, J. Deckert ${ }^{6}$, K. Domschke7, G. Eckermann ${ }^{8}$, K. Egberts $^{9}$,

M. Gerlach ${ }^{9}$, C. Greiner ${ }^{10}$, G. Gründer ${ }^{11}$, E. Haen ${ }^{12}$, U. Havemann-Reinecke ${ }^{13}$, G. Hefner ${ }^{14}$, R. Helmer ${ }^{15}$, G. Janssen ${ }^{16}$,

E. Jaquenoud ${ }^{17}$, G. Laux ${ }^{18}$, T. Messer ${ }^{19}$, R. Mössner ${ }^{20}$, M. J. Müller ${ }^{21}$, M. Paulzen ${ }^{11}$, B. Pfuhlmann22,

P. Riederer ${ }^{6}$, A. Saria ${ }^{23}$, B. Schoppek ${ }^{24}$, G. Schoretsanitis ${ }^{25}$, M. Schwarz ${ }^{26}$, M. Silva Gracia'2, B. Stegmann ${ }^{12}$,

W. Steimer ${ }^{27}$, J. C. Stingl ${ }^{10}$, M. Uhr ${ }^{28}$, S. Ulrich ${ }^{29}$, S. Unterecker ${ }^{6}$, R. Waschgler ${ }^{30}$, G. Zernig 23, 31, G. Zurek $^{32}$,

P. Baumann 33

Affiliations

1 Department of Psychiatry and Psychotherapy, University Medical Center of Mainz, Mainz, Germany

2 Institute of Clinical Chemistry and Laboratory Medicine, University Medical Center of Mainz, Mainz, Germany

3 Kitzberg Hospitals, Center for Psychosomatic Medicine and Psychotherapy, Bad Mergentheim, Germany

4 Department of Child and Adolescent Psychiatry, University of Freiburg, Freiburg, Germany

5 Servizio Psichiatrico del Comprensorio Sanitario di Bolzano, Bolzano, Italy

6 Department of Psychiatry, Psychotherapy and Psychosomatics, University Hospital of Würzburg, Germany

7 Department of Psychiatry and Psychotherapy, Medical Center - University of Freiburg, Faculty of Medicine, University of Freiburg, Freiburg, Germany

8 Psychiatric Hospital, Kaufbeuren, Germany

9 Department of Child and Adolescent Psychiatry, Psychosomatics and Psychotherapy, Center of Mental Health, University Hospital of Würzburg, Germany

10 Federal Institute for Drugs and Medical Devices (BfArM), Bonn, Germany

11 Department of Psychiatry, Psychotherapy and Psychosomatics, RWTH Aachen University, Aachen, and JARA Translational Brain Medicine, Aachen, Germany

12 Clinical Pharmacology, Department of Psychiatry and Psychotherapy and Department of Pharmacology and Toxicology, University of Regensburg, Regensburg, Germany

13 Department of Psychiatry and Psychosomatics, University of Göttingen, Göttingen, Germany

14 Psychiatric Hospital, Vitos Klinik, Eichberg, Eltville, Germany

15 Center of Epilepsy, Bielefeld, Germany

16 Medical Laboratory Stein, Limbach Group,

Mönchengladbach, Germany

17 Psychiatric Hospital, Königsfelden, Brugg, Aargau, Switzerland

18 Institute of Psychological Medicine, Haag in Oberbayern, Germany
19 Danuviuskliniken, Psychiatric Hospital, Pfaffenhofen, Germany

20 Department of Psychiatry and Psychotherapy, University of Tübingen, Tübingen, Germany

21 Psychiatric Hospitals Oberberggruppe, Berlin, Germany

22 Psychiatric Hospital Weisser Hirsch, Dresden, Germany

23 Experimental Psychiatry Unit, Department of Psychiatry 1, Medical University of Innsbruck, Innsbruck, Austria

24 kbo-Isar-Amper Klinikum München-Ost, Psychiatric Hospital, Munich-Haar, Germany

25 Department of Psychiatry, University of Bern, Bern, Switzerland

26 Department of Laboratory Medicine, Ludwig Maximilian University, Munich, Germany

27 Institute of Clinical Chemistry and Pathobiochemistry, Technical University Munich, Munich, Germany

28 Max Planck Institute of Psychiatry, Munich, Germany

29 Aristo Pharma GmbH, Berlin, Germany

30 Psychiatric Hospital, Feldkirch, Austria

31 Private Practice for Psychotherapy and Court-Certified Witness, Hall in Tirol, Austria

32 Medical Laboratory Bremen, Bremen, Germany

33 Department of Psychiatry, University of Lausanne, Prilly-Lausanne, Switzerland

Key words antidepressant drugs, antiepileptic drugs, antiparkinson drugs, antipsychotic drugs, concentration in blood, consensus guidelines, drug analysis, genotyping, neurologic drugs, pharmacogenetics, pharmacokinetics, phenotyping, plasma concentration, psychiatric drugs, reference range, serum concentration, therapeutic drug monitoring, therapeutic window

received 15.05 .2017

revised 08.07.2017

accepted 10.07.2017 


\section{Bibliography}

DOI http://dx.doi.org/10.1055/s-0043-116492

Published online: 14.9.2017

Pharmacopsychiatry 2018; 51: 9-62

(c) Georg Thieme Verlag KG Stuttgart · New York

ISSN 0176-3679

\section{Correspondence}

C. Hiemke, PhD, Univ.-Prof.

Department of Psychiatry and Psychotherapy

University Medical Center, Mainz

Untere Zahlbacher Str. 8

D-55101 Mainz

Germany

hiemke@uni-mainz.de

\section{ABSTRACT}

Therapeutic drug monitoring (TDM) is the quantification and interpretation of drug concentrations in blood to optimize pharmacotherapy. It considers the interindividual variability of pharmacokinetics and thus enables personalized pharmacotherapy. In psychiatry and neurology, patient populations that may particularly benefit from TDM are children and adolescents, pregnant women, elderly patients, individuals with intellectual disabilities, patients with substance abuse disorders, forensic psychiatric patients or patients with known or suspected pharmacokinetic abnormalities. Non-response at therapeutic doses, uncertain drug adherence, suboptimal tolerability, or pharmacokinetic drug-drug interactions are typical indications for TDM. However, the potential benefits of TDM to optimize pharmacotherapy can only be obtained if the method is adequately integrated in the clinical treatment process. To supply treating physicians and laboratories with valid information on TDM, the TDM task force of the Arbeitsgemeinschaft für Neuropsychopharmakologie und Pharmakopsychiatrie (AGNP) issued their first guidelines for TDM in psychiatry in 2004. After an update in 2011, it was time for the next update. Following the new guidelines holds the potential to improve neuropsychopharmacotherapy, accelerate the recovery of many patients, and reduce health care costs.

\section{Table of Contents}

Abbreviations

Background

Objectives of the Consensus Document

Preparation of the Consensus Document

1. Pharmacokinetics and pharmacogentics

1.1 Pharmacokinetic aspects

1.1.1 Absorption, distribution and elimination of neuropsychiatric drugs

1.1.2 Drug concentrations in blood

1.1.3 Drug concentrations in brain and cerebrospinal fluid

1.2 Pharmacogenetic aspects
2. Drug Concentrations in Blood to Guide

Neuropsychopharmacotherapy $\quad 22$

2.1 The therapeutic reference range 22

2.1.1 Estimation of the lower limit of the therapeutic reference range

2.1.2 Estimation of the upper limit of the therapeutic reference range

2.1.3 From population-based to subject-based reference values

2.1.4 Laboratory alert level

The dose-related reference range

Concentration to dose ratio

Metabolite to parent compound ratios

Probe drug phenotyping

Indications for measuring drug concentrations in blood

Recommendations for measuring drug

concentrations in blood

\section{Abbreviations}

BBB blood-brain-barrier

C/D concentration to dose ratio

$\mathrm{CL}$ total clearance

$\mathrm{CL} / \mathrm{F}$ apparent total clearance

Cav average concentration

Cmax maximal concentration

Cmin trough or minimal concentration

CYP cytochrome P450

DRC dose-related concentration

EMA European Medicines Agency

$\mathrm{F}$

FDA

GeKO

MPR

PET

P-gp

bioavailability (fraction absorbed)

Food and Drug Administration

German Commission Genetic Testing

metabolite to parent compound ratio

positron emission tomography

P-glycoprotein

$\mathrm{t} 1 / 2$ elimination half-life

12 TDM

therapeutic drug monitoring

tmax

time of maximal drug concentrations

tmin 


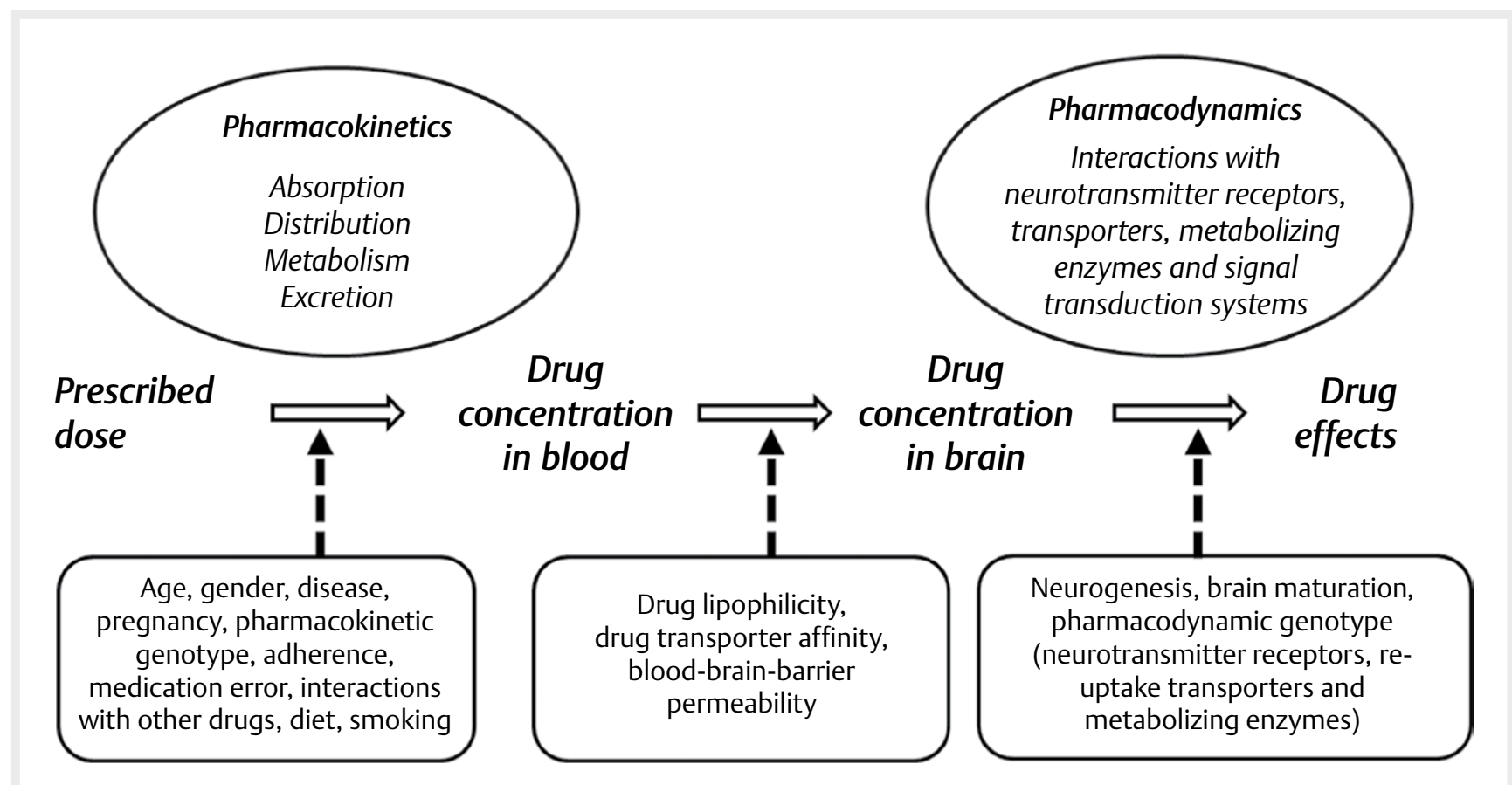

Fig. 1 From prescribed dose to drug effects modulated by multiple factors leading to marked pharmacokinetic and pharmacodynamic variability.

\section{Background}

For the treatment of psychiatric and neurologic patients, more than 200 drugs are available which have been discovered and developed during the last 60 years [89]. These drugs are effective and essential for the treatment of many neuropsychiatric/mental disorders and symptoms. Despite enormous medical and economic benefits, however, therapeutic outcomes are still far from satisfactory for patients and the prescribing physicians $[6,8,709,1206]$. Therefore, after having focused clinical research on the development of new drugs $[953,954]$, growing evidence suggests that an improved application of available drugs may still bring substantial benefit to patients [75, 190, 248, 267, 1080]. Moreover, there is a gap between the available pharmacologic knowledge and its utilization in health care [1094]. The newest initiative to bridge this gap is "Precision Medicine". It considers individual variability to build the evidence base needed to guide clinical practice [229]. Therapeutic drug monitoring (TDM) is a patient management tool for precision medicine [565]. It enables tailoring the dosage of the medication(s) to the individual patient by combining the quantification of drug concentrations in blood, information on drug properties and patient characteristics. One major reason to use TDM for the guidance of neuropsychopharmacotherapy is the interindividual pharmacokinetic variability of the drugs in patients $[957,960]$. At the very same dose, a more than 20-fold interindividual variation in the drug's steady-state concentration in the body may result, as patients differ in their ability to absorb, distribute, metabolize and excrete drugs due to concurrent disease, age, concomitant medication or genetic abnormalities [96, 328, 518, 520, 568, 569, 651]. Different pharmaceutic formulations of the same drug may also influence the degree and temporal pattern of absorption and, hence, drug concentrations in the body ( $\triangleright \mathbf{F i g}$. 1). TDM uses the quantification of a drug's concentration in blood plasma or serum to titrate the dosage of individual patients to a drug concentration in blood that is associated with the highest possible probability of response and a low risk of adverse drug reactions/toxicity. Moreover, TDM has the potential to enhance cost-effectiveness of neuropsychopharmacotherapy [13, 894, 961, 1204, 1267]. Despite TDM's potential, considerable disagreement was found between the information on TDM in official product information and existing medico-scientific evidence. Even for well-studied compounds, such as amitriptyline or clozapine, insufficient information on TDM was found in the product information (Summary of Product Characteristics, SPC) [1020, 1221]. For a large number of neuropsychopharmacological drugs, however, the quantification of blood concentrations has become clinical routine. Clear evidence of the benefits of TDM has been demonstrated for anticonvulsant drugs [912], tricyclic antidepressants [826], old (first generation or "typical") and new (second generation or "atypical") antipsychotic drugs [928] and mood stabilizing drugs [233]. For the mood stabilizer lithium, TDM has become a standard of care due to its narrow therapeutic range [230, 463, 707].

The benefits of TDM for optimization of pharmacotherapy, however, can only be obtained when the method is adequately integrated in the clinical treatment process. Current TDM use in neuropsychiatric care is often suboptimal as demonstrated by systematic studies [231,462, $725,1077,1272,1346]$. The suboptimal use of TDM wastes laboratory resources and bears the risk of misleading results that will adversely influence clinical decision making [204]. A study on the clinical use of TDM for tricyclic antidepressants in psychiatric university hospital settings showed that 25 to $40 \%$ of the requests for TDM were insufficiently filled out. Misinterpretation of the results led to about $20 \%$ of incorrect dosage adjustments [1272, 1346, 1347]. Other typical errors were absence of steady-state conditions at the time of blood sampling and transcrip- 
tion errors on the request form. Studies on TDM for antidepressant and mood stabilizing drugs further specified the information on the imperfect use of TDM [757, 758]. For antiepileptic drugs, it was found that half of all requisitions were inappropriate [1077].

Against this background, the TDM task force of the working group on neuropsychopharmacology (Arbeitsgemeinschaft fuer Neuropsychopharmakologie und Pharmakopsychiatrie, AGNP) issued best practice guidelines for TDM in psychiatry with inclusion of recommendations for genotyping in 2004 [82]. In 2011, the guidelines were updated and considerably extended to include a large number of additional drugs, especially neurologic medications [524]. These guidelines were widely accepted by laboratories and practicing clinicians. The first guidelines [82] have been cited more than 300 times in the literature [1048]. The guidelines were translated into German [453, 521], Hungarian [523], French [85], Italian [522] and Chinese. Since 2011, knowledge about and acceptance of TDM has further advanced. The TDM task force of the AGNP therefore prepared this second updated version.

\section{Objectives of the Consensus Document}

This document addresses topics related to the theory and practice of TDM in psychiatry and neurology. The first part deals with theoretical aspects of monitoring neuropsychiatric drug concentrations in blood. The second part defines indications for TDM and gives orienting therapeutic concentrations in blood for dosage optimization. The third part describes best practice TDM, a process that starts with a request and ends in a clinical decision to either continue or change the pre-TDM pharmacotherapy.

To optimize the practice of TDM the following topics are addressed:

- definition of indications for using TDM in psychiatry and neurology

- definition of levels of recommendations to use TDM

- definition of therapeutic and dose-related reference ranges that laboratories can quote and clinicians can use to guide pharmacotherapy

- definition of alert levels for laboratories to warn the treating physician when drug concentrations are considered to be too high and potentially harmful

- recommendations and help for interpretative services

- recommendations for the combination of TDM with pharmacogenetic tests

- presentation of pharmacokinetic parameters required for interpretation of TDM results

\section{Preparation of the Consensus Document}

The updated consensus guidelines were prepared by the interdisciplinary TDM task force of the AGNP consisting of psychiatrists, neurologists, psychotherapists, pharmacologists including a court-certified pharmacology expert, biochemists, pharmacists and chemists from university hospitals and hospitals/institutions almost exclusively concerned with patient care in Germany, Switzerland, Austria, and Italy.

Data published in the previous AGNP consensus guidelines [82,524] and other guidelines and recommendations for TDM of neuropsychiatric drugs $[536,587,715,869,889-891,912,928,932,1304]$ were used. A systematic literature search was conducted, primarily in PubMed and in summaries of product characteristics (SPC), and also by hand in pharmacologic and clinical chemical journals to identify TDM-related information. More than two thousand articles were assessed. Finally, data were extracted from around 1400 articles identified as relevant for this 2 nd update. A checklist (drug AND concentration AND (blood OR plasma OR serum)) was used to extract and analyse reported data. The search focused on therapeutic and dose-related drug concentrations in serum, plasma or blood. For the interpretative service of TDM, information on cytochrome P450 (CYP) substrate properties and metabolite parent compound ratios (MPR) were adopted or newly calculated. Moreover, CYP inducing and inhibiting properties of drugs and food constituents that are potentially relevant for pharmacokinetic drug-drug interactions were searched. Final decisions on the data presented in this update were made during five consensus conferences and by e-mail communication.

Therapeutic reference ranges are now listed for 154 neuropsychiatric drugs. Reference ranges were newly introduced for 25 drugs (levomilnacipran, tianeptine, vilazodone, vortioxetine, brexpiprazole, cariprazine, loxapine, lurasidone, $\mathrm{N}$-desalkylquetiapine, brivaracetam, eslicarbazepine, perampanel, retigabine, diphenhydramine, doxylamine, gamma-hydroxy butyric acid, medazepam, modafinil, promethazine, zaleplone, heroin, morphine, nalmefene, nicotine and rotigotine) and revised for 18 drugs (bupropion, milnacipran, paroxetine, aripiprazole, asenapine, flupentixol, prothipendyl, felbamate, topiramate, lorazepam, temazepam, zolpidem, donepezil, galantamine, buprenorphine, disulfiram, methylphenidate and 3-O-methyldopa).

Special attention was given to the calculation of dose-related concentration (DRC) factors to compute dose-related reference ranges. They are used independently of the therapeutic reference range to identify adherence problems as well as individual pharmacokinetic abnormalities due to drug-drug interactions, poor or ultrarapid drug metabolism or altered liver or kidney function. The concept was introduced by Haen and colleagues [471] and adopted in the consensus guidelines 2011 for 83 neuropsychiatric drugs [524]. It was revised for this update and extended to 133 neuropsychiatric drugs, for 29 with inclusion of metabolites.

\section{Pharmacokinetics and pharmacogenetics 1.1 Pharmacokinetic aspects}

1.1.1 Absorption, distribution and elimination of neuropsychiatric drugs

Most neuropsychiatric drugs share a number of pharmacokinetic characteristics

- good absorption from the gastrointestinal tract into the blood compartment reaching maximal concentrations within 1-6h

- highly variable systemic bioavailability ranging from 5 to essentially $100 \%$

- fast distribution from the blood compartment to the central nervous system with mostly higher levels in brain than in blood

- high apparent volume of distribution (about 10-50 L/kg)

- low trough drug concentrations in blood under steady-state conditions (about $0.1-500 \mathrm{ng} / \mathrm{mL}$ for psychiatric drugs and up to $20 \mu \mathrm{g} / \mathrm{mL}$ for neurologic drugs)

- elimination mainly by hepatic metabolism

- elimination half-life mostly between 12-36 h 
- linear pharmacokinetics at therapeutic doses with the consequence that doubling the daily dose will result in doubling the drug concentration in blood

- cytochrome P450 (CYP) and UDP-glucuronosyltransferases

(UGT) as major metabolic enzyme systems

There are, however, numerous exceptions to this list of common pharmacokinetic features. For example, agomelatine, venlafaxine, trazodone, tranylcypromine, moclobemide, quetiapine, rivastigmine or ziprasidone display short (about 2-10 h) elimination halflives, whereas aripiprazole and fluoxetine have long elimination half-lives ( $72 \mathrm{~h}$ for aripiprazole and 3-15 days for fluoxetine, taking into account its active metabolite norfluoxetine). Amisulpride, milnacipran, memantine, gabapentin, or sulpiride are only poorly metabolized in the liver and mainly excreted renally which may be advantageous for patients with impaired liver function. Paroxetine exhibits non-linear pharmacokinetics, due to inhibition of its own metabolism by a metabolite which is irreversibly bound to the enzyme resulting in its inactivation [108].

Many neuropsychopharmacological drugs are used as racemic compounds, and their enantiomers differ markedly in their pharmacodynamic and pharmacokinetic properties [88, 1104]. So far, however, methadone and methylphenidate are at present the only racemic psychotropic compounds for which TDM of the enantiomers has been introduced [68, 322]. The active enantiomer of racemic methadone is (R)-methadone, and I-methylphenidate (i. e., levorotary methylphendiate) is primarily responsible for the therapeutic effect of racemic methylphenidate. Flupentixol is available as a 1:1 mixture of the geometric cis- and trans-isomers ( $\mathrm{Z}$ - and $\mathrm{E}$ isomers, respectively) for oral administration, while the depot preparation flupentixol decanoate contains exclusively cis-flupentixol. Only the latter is considered to be pharmacologically active with regard to its affinity for dopamine (and serotonin) receptors, as shown in clinical studies in which clinical efficacy of cis-flupentixol ( $\alpha$-flupentixol; Z-flupentixol) was found to be superior to that of trans-flupentixol [83]. For research projects and other special situations, stereoselective analysis should be considered for parent drugs and/or metabolites, e. g., for citalopram, fluoxetine, venlafaxine, paliperidone or amitriptyline.

Inter- and intra-individual differences in blood concentrations of neuropsychopharmacological drugs (i.e., the pharmacokinetic variability) are caused by different activities of drug-metabolizing enzymes. The enzyme activity may decrease with age [651] and can be modified by renal and hepatic diseases. Most psychiatric or neurologic drugs undergo phase 1 metabolism by oxidative (e. g., hydroxylation, dealkylation, oxidation to $\mathrm{N}$-oxides, $\mathrm{S}$-oxidation to sulfoxides or sulfones), reductive (e. g., carbonyl reduction to secondary alcohols) or hydrolytic reactions [81]. Phase 1 reactions are predominantly catalyzed by CYP enzymes. They are proteins of a superfamily containing heme as a cofactor and function as terminal oxidases in electron transfer chains. The term P450 is derived from the spectrophotometric peak at the wavelength of the absorption maximum of the CYP enzymes $(450 \mathrm{~nm})$ in their reduced state complexed with carbon monoxide. CYP-catalyzed phase 1 reactions introduce a polar functional group that enables a phase 2 conjugation reaction with highly polar molecules such as glucuronic or sulphuric acid. For neuropsychopharmacological drugs possessing functional groups in the parent compound, glucuronida- tion of a hydroxyl (for example oxazepam or lorazepam) or an amine group to form $\mathrm{N}$-glucuronides (for example olanzapine) may represent the essential metabolic pathway. According to their primary structure (sequence of amino acids) they are classified in 18 families of CYP genes and 43 subfamilies. In humans, 57 putatively functional genes and 58 pseudogenes are encoded by various gene clusters [1344]. For neuropsychopharmacological drugs, the most important isoenzymes are CYP1A2, CYP2B6, CYP2C8, CYP2C9, CYP2C19, CYP2D6, CYP2E1 and CYP3A4/5 ( Table 1) [59, 1344, 1351-1353]. Many CYP genes are highly susceptible to mutation. As explained below in more detail, genetic polymorphisms of CYP enzymes are major causes for the large interindividual variability of drug concentrations in the body, which gives rise to the need to measure them in blood.

Other enzymes may also be metabolic key determinants of drug action and toxicity [73]. Enzymes, such as aldo-keto reductases (AKRs), of the AKR superfamily catalyze reduction of aldehyde or ketone groups of endo- and exogenous compounds. In humans, 13 AKR proteins have been identified [73]. It was shown that they reduce ziprasidone to its dihydro derivative [93] and naltrexone to naltrexol [152]. Monoamine oxidase subtypes A and B (MAO-A and MAO-B) deaminate citalopram stereoselectively to an apparently inactive acidic metabolite [1007].

Actually, phase 2 enzymes are increasingly characterised with regard to substrate specificity. There is much overlap between the isoenzymes regarding affinity for substrates [245, 878]. Consequences for TDM are so far unclear.

Drugs are metabolized mainly in the liver and, to a minor degree, in extrahepatic tissues such as the intestinal mucosa or the brain [94, 402, 803].

When combining drugs that are inhibitors or inducers of drug metabolizing enzymes ( $\triangleright$ Table 2, 3), pharmacokinetic drug-drug interactions may occur if the comedication is a substrate of the inhibited or induced enzyme. Many interactions have been found by TDM either by chance or retrospective analysis of TDM data bases $[183,502,918,974,1054,1055,1295]$. Among environmental factors, smoking is of high clinical relevance for drugs that are substrates of CYP1A2 [336, 343]. CYP1A2 is dose-dependently induced by constituents of cigarette smoke (polycyclic aromatic hydrocarbons, not nicotine). When smoking 1-5, 6-10 and > 10 cigarettes per day, the activity of CYP1A2 increases by 1.2-, 1.5- and 1.7-fold, respectively [342]. The increased activity returns to baseline within three days after smoking cessation. Smoking effects should therefore be considered at least when more than 10 cigarettes are smoked per day [343]. Cessation of heavy smoking under therapy with a CYP1A2 substrate ( Table 1) such as clozapine [133, 1232], duloxetine [375] or olanzapine [1357] may require dose reduction which should be controlled by TDM.

Besides enzymes involved in phase 1 and 2 metabolism, drug transporters play a role in the distribution pharmacokinetics of drugs $[161,301,1214,1320]$. They are ATP-binding cassette (ABC) proteins located in cell membranes and function as efflux transporters to protect organs against xenobiotics. For many neuropsychopharmacological drugs, ABC transporters, especially P-glycoprotein ( $P$-gp), the gene product of $A B C B 1$, multidrug resistance protein (MRP) encoded by $A B C C 1$ and breast cancer resistance protein (BCRP) encoded by ABCG2 have been identified as major de- 
- Table 1 Enzymes and efflux transporters involved in the metabolism and distribution of neuropsychopharmacological compounds.

\begin{tabular}{|c|c|c|}
\hline Drugs & Enzymes and transporters & References \\
\hline Acamprosate & Not metabolized & [1033] \\
\hline Agomelatine & CYP1A2, CYP2C19, CYP3A4 & {$[126,721]$} \\
\hline Alprazolam & CYP3A4 & {$[24,905]$} \\
\hline Amantadine & $90 \%$ is excreted unchanged via the kidney & [38] \\
\hline Amisulpride & More than $90 \%$ is excreted unchanged via the kidney & [1018] \\
\hline Amitriptyline & $\begin{array}{l}\text { CYP1A2, CYP2C9, CYP2C19, CYP2D6, CYP3A4, UGT1A3, } \\
\text { UGT1A4, UGT2B10, P-gP (ABCB1) }\end{array}$ & $\begin{array}{l}{[84,150,516,878,1187,1215,1216,} \\
1293]\end{array}$ \\
\hline Amitriptyline oxide & FMO, CYP2C19, CYP2D6 & {$[150,276]$} \\
\hline $\begin{array}{l}\text { Amfetamine (dexamfetamine, } \\
\text { lisdexamfetamine) }\end{array}$ & CYP2D6 & [55] \\
\hline Aripiprazole & CYP2D6, CYP3A4, P-gp (ABCB1) & {$[509,639,832,1273]$} \\
\hline Asenapine & CYP1A2, UGT1A4 & {$[222,1285]$} \\
\hline Atomoxetine & CYP2C19, CYP2D6, P-gp (ABCB1) & {$[217,805,1354]$} \\
\hline Benperidol & Unknown & [1068] \\
\hline Benserazide & Hydroxylation, COMT & [594] \\
\hline Biperiden & Unknown & [1146] \\
\hline Brexpiprazole & CYP3A4, CYP2D6 & [443] \\
\hline Brivaracetam & CYP2C8, renal elimination & [1042] \\
\hline Bromazepam & CYP2C19, CYP3A4 & {$[26,877]$} \\
\hline Bromocriptine & CYP3A4 & [938] \\
\hline Bromperidol & CYP3A4 & {$[388,1156,1176,1337]$} \\
\hline Brotizolam & CYP3A4 & [1193] \\
\hline Buprenorphine & CYP2C8, CYP3A4, UGT1A3, UGT2B7 & {$[129,817]$} \\
\hline Bupropion & CYP2C19, CYP2B6, CR & {$[232,514]$} \\
\hline Buspirone & CYP3A4 & [748] \\
\hline Cabergoline & Unknown, CYP3A4, P-gp (ABCB1) & {$[54,278]$} \\
\hline Caffeine & CYP1A2, CYP2A6, xanthine oxidase, NAT & {$[15,386,475]$} \\
\hline Carbamazepine & $\begin{array}{l}\text { CYP1A2, CYP2C8, CYP3A4, UGT2B7, P-gp (ABCB1), BCRP } \\
\text { (ABCG2), epoxide hydrolase }\end{array}$ & {$[586,618,730,906,1214,1280]$} \\
\hline Carbidopa & Loss of the functional hydrazine group, $1 / 3$ not metabolized & {$[1030,1261]$} \\
\hline Cariprazine & CYP2D6, CYP3A4 & {$[174,840]$} \\
\hline Chlordiazepoxide & CYP3A4 & SPC \\
\hline Chlorpromazine & CYP1A2, CYP2D6, P-gp (ABCB1) & {$[1277,1316]$} \\
\hline Chlorprothixene & Probably CYP2D6, CYP3A4 & \\
\hline Citalopram & CYP2C19, CYP2D6, CYP3A4, P-gp (ABCB1) & {$[158,384,1339]$} \\
\hline Clobazam (norclobazam) & CYP2C19, CYP3A4 & {$[271]$} \\
\hline Clomethiazole & CYP2A6, CYP3A4 & [189] \\
\hline Clomipramine & CYP1A2, CYP2C19, CYP2D6, CYP3A4, UGT2B10 & {$[412,878]$} \\
\hline Clonazepam & CYP3A4 & [1070] \\
\hline Clorazepate & CYP2C19, CYP3A4 & \\
\hline Clozapine & CYP1A2, CYP2C19, CYP3A4, P-gp (ABCB1) & {$[568,884,1232,1278]$} \\
\hline Cocain & Carboxylesterase 1 and 2, pseudocholinesterase, CYP3A4 & [776] \\
\hline Codeine & CYP2D6, CYP3A4, UGT2B4, UGT2B7 & {$[802,878]$} \\
\hline Cyamemazine & CYP1A2, CYP2C8, CYP2C9, CYP2C19, CYP3A4 & {$[45]$} \\
\hline Dapoxetine & CYP2D6 & [1333] \\
\hline Desipramine & CYP2D6 & [412] \\
\hline Desvenlafaxine & CYP3A4, CYP2C19, UGT & [63] \\
\hline Dextroamfetamine & CPY2D6 & [55] \\
\hline Diacetylmorphine (heroin) & Carboxylesterase 2 and 1, UGT & {$[776,802,878]$} \\
\hline Diazepam & CYP2B6, CYP2C19, CYP3A4, UGT2B7, P-gp (ABCB1) & {$[387,846,1275]$} \\
\hline Dihydroergocryptine & CYP3A4 & {$[29,274]$} \\
\hline Diphenhydramine & CYP2D6, UGT1A4, UGT2B10, P-gp (ABCB1) & {$[17,846,878]$} \\
\hline
\end{tabular}


- Table 1 Continued.

\begin{tabular}{|c|c|c|}
\hline Drugs & Enzymes and transporters & References \\
\hline Disulfiram & CYP1A2, CYP2A6, CYP2B6, CYP2E1, CYP3A4 & [743] \\
\hline Donepezil & CYP2D6, CYP3A4, P-gp (ABCB1) & {$[863,1242]$} \\
\hline Dothiepin (dosulepin) & CYP2C19, CYP2D6 & [1341] \\
\hline Doxepin & CYP2C9, CYP2C19, CYP2D6 & {$[488,633]$} \\
\hline Doxylamine & Unknown & \\
\hline Dronabinol & CYP2C9, CYP3A4, UGT1A9, UGT1A7, UGT1A8, UGT1A10 & {$[119,787,878]$} \\
\hline Duloxetine & CYP1A2, CYP2D6, P-gp (ABCB1) & {$[726,1024]$} \\
\hline Entacapone & UGT1A9 & [687] \\
\hline Escitalopram & CYP2C19, CYP2D6, CYP3A4, P-gp (ABCB1) & {$[166,1207,1268]$} \\
\hline Ethanol & Alcohol dehydrogenase, CYP2E1 & [193] \\
\hline Felbamate & Renal excretion & [995] ] \\
\hline Flunitrazepam & CYP2C19, CYP3A4 & {$[228,392]$} \\
\hline Flunarizine & CYP2D6 & [841] \\
\hline Fluoxetine & CYP2B6, CYP2C9, CYP2C19, CYP2D6, P-gp (ABCB1) & {$[723,1064]$} \\
\hline Flupenthixol & CYP2D6 & {$[254,633]$} \\
\hline Fluphenazine & CYP2D6, P-gp (ABCB1) & [1352-1353] \\
\hline Flurazepam & CYP2C19, CYP3A4 & [1061] \\
\hline Fluspirilene & Renal excretion, CYP3A4 & [1113] \\
\hline Fluvoxamine & CYP2D6, CYP1A2, P-gp (ABCB1) & {$[303,611,814]$} \\
\hline Gabapentin & Not metabolized, renal excretion & [123] \\
\hline Galantamine & CYP2D6, CYP3A4 & {$[58]$} \\
\hline Gammahydroxybutyric acid (GHB) & Beta-oxidation & [710] \\
\hline Guanfacine & CYP3A4, epoxide hydratase, UGT & [623] \\
\hline Haloperidol & CYP2D6, CYP3A4, AKR, UGT, P-gP (ABCB1) & {$[73,154,1176,1277]$} \\
\hline Heroin (diacetylmorphine) & Carboxylesterase 2 and 1, UGT & {$[776,802,878]$} \\
\hline Iloperidone & CYP2D6, CYP3A4 & [175] \\
\hline Imipramine & CYP1A2, CYP2C19, CYP2D6, CYP3A4, UGT1A4, UGT2B10 & {$[412,744,878]$} \\
\hline Lamotrigine & UGT1A4, UGT3B7, P-gP (ABCB1), BCRP (ABCG2) & {$[201,1281]$} \\
\hline Levetiracetam & Not metabolized, P-gp (ABCB1) & [849] \\
\hline Levodopa & DDC, COMT, MAO & {$[1030]$} \\
\hline Levomepromazine & CYP3A & {$[61,1315]$} \\
\hline Levomilnacipran & CYP3A4, P-gp (ABCB1) & {$[166,901]$} \\
\hline Levomethadone & CYP2B6, CYP3A4, CYP2D6 & [249] \\
\hline Levosulpiride & P-gp (ABCB1) & {$[214]$} \\
\hline Lisdexamfetamine & Erythrocyte peptidase, CYP2D6 & [668] \\
\hline Lisuride & CYP3A4, CYP2D6 & [975] \\
\hline Lithium & Renal clearance & {$[424,1125]$} \\
\hline Lorazepam & UGT2B15 & {$[275,334,878]$} \\
\hline Loxapine & CYP3A4, CYP2D6, CYP1A2, CYP2C8, CYP2C19, FMO & [736] \\
\hline Lurasidone & CYP3A4 & [213] \\
\hline Maprotiline & CYP2D6, CYP1A2 & [140] \\
\hline Medazepam & CYP2B6, CYP2C19, CYP3A4 & SPC \\
\hline Melatonin & CYP1A2 & [489] \\
\hline Melperone & Unknown & [135] \\
\hline Memantine & Scarcely metabolized & [419] \\
\hline Methadone & CYP2B6, CYP3A4, CYP2D6, ABCB1 & {$[249,718,1082,1203]$} \\
\hline Methylphenidate & Carboxylesterase 1 & [844] \\
\hline Mianserin & CYP2D6, CYP1A2, CYP3A4 & [664] \\
\hline Midazolam & CYP3A4, UGT1A4 & {$[372,878]$} \\
\hline Milnacipran & $C Y P 3 A 4, A B C B 1$, renal excretion & {$[166,704,904,968]$} \\
\hline Mirtazapine & CYP3A4, CYP1A2, CYP2D6 & {$[712,1150]$} \\
\hline
\end{tabular}


- Table 1 Continued.

\begin{tabular}{|c|c|c|}
\hline Drugs & Enzymes and transporters & References \\
\hline Moclobemide & CYP2C19, CYP2D6 & {$[423]$} \\
\hline Modafinil & Amide hydrolase, CYP3A4 & {$[1003,1323-1324]$} \\
\hline Morphine & CYP2C8, CYP3A4, UGT2B7 & {$[262,620,776]$} \\
\hline Nalmefene & UGT & [297] \\
\hline Naloxone & UGT2B7, AKR1C & {$[73,878]$} \\
\hline Naltrexone & AKR1C4 & {$[73,152]$} \\
\hline Nicotine & CYP2A6, UGT1A1, UGT1A2, UGT2B10 & {$[104]$} \\
\hline Nitrazepam & CYP3A4 & {$[1171]$} \\
\hline Nordazepam & CYP3A4, CYP2C19 & {$[887,1171]$} \\
\hline Nortriptyline & CYP2D6, P-gp (ABCB1) & {$[675,885,1215,1249]$} \\
\hline Olanzapine & UGT1A4, UGT2B10, FMO, CYP1A2, CYP2D6, P-gp (ABCB1) & {$[176,337,878,1277]$} \\
\hline Opipramol & CYP2D6 & SPC \\
\hline Oxazepam & UGT1A9, UGT2B7, UGT2B15 & {$[246,878]$} \\
\hline Oxcarbazepine & AKR, UGT2B15, P-gp (ABCB1) & {$[73,878,1279]$} \\
\hline Paliperidone (9-hydroxyrisperidone) & $\begin{array}{l}60 \% \text { excreted unmetabolized, CYP3A4, UGT, P-gp (ABCB1), } \\
\text { BCRP (ABCG2) }\end{array}$ & {$[273,303,461,1250,1277-1278]$} \\
\hline Paroxetine & CYP2D6, CYP3A4, P-gp (ABCB1) & {$[303,351,596,1215,1256]$} \\
\hline Perampanel & CYP3A4, CYP2B6, UGT1A1, UGT1A4 & [910] \\
\hline Perazine & CYP1A2, CYP2C9, CYP2C19, CYP3A4, FMO & {$[1149,1316]$} \\
\hline Pergolide & CYP3A4 & [1329] \\
\hline Perphenazine & CYP1A2, CYP2C19, CYP2D6, CYP3A4 & {$[16,886]$} \\
\hline Phenytoin & CYP2C9, CYP2C19, UGT2B15 & [730] \\
\hline Phenobarbital & CYP2C19, UGT1A4 & {$[34]$} \\
\hline Pimozide & CYP1A2, CYP2D6, CYP3A4 & {$[285,1011]$} \\
\hline Pipamperone & Unknown & \\
\hline Piribedil & Demethylation, p-hydroxylation, and $\mathrm{N}$-oxidation & [279] \\
\hline Pramipexole & Not metabolized & [97] \\
\hline Prazepam & CYP2C19, CYP3A4 & SPC \\
\hline Pregabalin & Not metabolized, renal excretion & {$[123]$} \\
\hline Promazine & CYP1A2, CYP2A6, CYP2C19, CYP3A4 & [1318] \\
\hline Promethazine & CYP2D6 & [839] \\
\hline Quetiapine & CYP3A4, CYP2D6, P-gp (ABCB1) & {$[65,1277]$} \\
\hline Rasagiline & CYP1A2 & [458] \\
\hline Reboxetine & CYP3A4 & {$[510,1299]$} \\
\hline Retigabine & NAT, UGT & [1197] \\
\hline Risperidone & CYP2D6, CYP3A4, P-gP (ABCB1), BCRP (ABCG2) & {$[303,461,1278,1330]$} \\
\hline Rivastigmine & Cholinesterase & \\
\hline Ropinirole & CYP1A2 & {$[614]$} \\
\hline Rotigotine & $\begin{array}{l}\text { CYP2C19, CYP1A1, CYP1A2, CYP2D6, CYP3A4, SULT1A1, } \\
\text { SULT1A2, SULT1A3, SULT1B1, SULT1C4, SULT1E1, UGT }\end{array}$ & {$[187,279,335,579]$} \\
\hline Rufinamide & Carboxylesterase & [936] \\
\hline Selegiline & CYP2B6 & [95] \\
\hline Sertindole & CYP2D6, CYP3A4 & [1322] \\
\hline Sertraline & $\begin{array}{l}\text { CYP2B6, CYP2C19, CYP2C9, CYP2D6, CYP3A4, UGT1A1, P-gP } \\
\text { (ABCB1) }\end{array}$ & {$[876,1215,1276]$} \\
\hline Sulpiride & Not metabolized, renal excretion, P-gp (ABCB1) & {$[214]$} \\
\hline Temazepam & CYP219, UGT2B7 & {$[622,887]$} \\
\hline Tetrahydrocannabinol (THC) & CYP2C9, CYP3A4 & {$[776,1151]$} \\
\hline Thioridazine & CYP1A2, CYP2D6, CYP3A4 & {$[1184,1294]$} \\
\hline Tianeptine & Beta-oxidation & [449] \\
\hline Tiapride & Not metabolized & [865] \\
\hline
\end{tabular}


- Table 1 Continued.

\begin{tabular}{|c|c|c|}
\hline Drugs & Enzymes and transporters & References \\
\hline Tolcapone & COMT, CYP2A6, CYP3A4, UGT & [687] \\
\hline Topiramate & UGT, P-gp (ABCB1) & {$[730]$} \\
\hline Tranylcypromine & MAO, unclear & {$[64]$} \\
\hline Trazodone & CYP3A4, CYP2D6 & {$[442,1019]$} \\
\hline Triazolam & CYP3A4 & [439] \\
\hline Trifluoperazine & UGT1A4 & [878] \\
\hline Trimipramine & CYP2C19, CYP2D6, CYP2C9, CYP3A4, UGT2B10 & {$[319,878]$} \\
\hline Valproic acid & $\begin{array}{l}\text { UGT1A3, UGT1A6, UGT2B7, CYP2A6, CYP2B6, CYP2C9, CYP219, } \\
\text { beta-oxidation }\end{array}$ & {$[878,1169]$} \\
\hline Venlafaxine & CYP2C19, CYP2D6, CYP2C9, CYP3A4, P-gp (ABCB1) & {$[367,606,788]$} \\
\hline Vilazodone & CYP3A4, P-gp (ABCB1) & {$[128,166]$} \\
\hline Vortioxetine & CYP2D6, CYP3A4, CYP2A6, CYP2C9, P-gP (ABCB1) & {$[548]$} \\
\hline Zaleplone & Aldehyde oxidase, CYP3A4 & [993] \\
\hline Ziprasidone & CYP3A4, aldehyde oxidase & {$[93,950]$} \\
\hline Zolpidem & CYP1A2, CYP2C9, CYP3A4 & [1269] \\
\hline Zopiclone & CYP2C8, CYP3A4 & {$[92,1202]$} \\
\hline Zotepine & CYP1A2, CYP2D6, CYP3A4 & [1083] \\
\hline Zuclopenthixol & CYP2D6 & [559] \\
\hline \multicolumn{3}{|c|}{$\begin{array}{l}\text { ABC: ATP-binding cassette; AKR: aldo-keto reductase; COMT: catechol-O-methyltransferase; CR: carbonyl reductase; CYP: cytochrome P450; DDC: } \\
\text { dopadecarboxylase (= aromatic amino acid decarboxylase); FMO: flavin monooxygenase; MAO: monoamine oxidase; NAT: N-acetyltransferase; SPC: } \\
\text { summary of product characteristics; SULT: sulfotransferase; UGT: UDP-glucuronosyltransferase; P-glycoprotein (P-gP) is encoded by the ABCB1 gene } \\
\text { and breast cancer resistance protein (BCRP) by the ABCG2 gene. Indicated CYP substrate properties are based primarily on in vivo studies in humans, } \\
\text { whereas ABC substrate properties rely on animal or cell line studies. When compounds are combined with strong or moderate inhibitors (See }>\text { Table } \\
\text { 2) or inducers (See — Table 3) and enzymes are indicated in bold, then the compounds' concentrations in blood will significantly increase or decrease. }\end{array}$} \\
\hline
\end{tabular}

terminants of drug distribution kinetics ( $\triangleright$ Table 1) [1320]. Drugs that are $A B C$ transporter substrates are taken up by passive diffusion into cells and then expelled via $A B C$ transporters into the extracellular space by ATP-dependent conformational changes. P-gP is highly expressed in the blood brain barrier (BBB) and the small intestine and thus plays a significant role in governing drug trafficking into and out of distinct organs [1320]. Animal studies give evidence that P-gp controls the availability rate of many antidepressant and antipsychotic drugs like nortriptyline, citalopram or risperidone in the brain $[303,1157,1215]$. It is suggested that high $\mathrm{P}$-gp function is responsible for inefficacious concentrations, and low P-gp function is associated with high drug concentrations and tolerability problems [111, 146, 147, 160, 263, 850, 978, 1217]. Similar to CYP enzymes, multiple genetic mutations have been identified for $A B C$ transporters [1320]. Moreover, the expression of $A B C$ transporters is up- and down-regulated in a variety of ways, e. g., by pathophysiological stressors, xenobiotics, hormones or dietary factors [809].

Gender differences have also been reported for the pharmacokinetics of neuropsychopharmacological drugs [9-11, 762, 1088, $1107,1127,1340]$, most likely due to effects of female sex hormones on the pharmacokinetic processes of absorption, distribution, metabolism, and excretion [256,656]. However, findings are still inconsistent and their clinical relevance is not yet clear. Although body weight should, on pharmacokinetic principle [77], be a major determinant of the blood concentration of a medication after administration of a certain dose, some studies found the impact of body weight to be less than predicted by pharmacokinetic principles $[9,1088,1226]$. Systematic research in these fields is still required.

\subsubsection{Drug concentrations in blood}

- Fig. 2 shows the concentration time curve after oral application of a hypothetical drug. At steady-state, drug intake equals drug elimination over a defined time frame. Concentrations will fluctuate during the day, especially in the case of drugs with short elimination half-lives ( $<12 \mathrm{~h}$ ) and depending on the dosing scheme (i.e., dosage) which must be considered for interpretations of TDM results [1134]. In TDM, trough concentrations (Cmin) at steady-state (therapy with constant dose for at least 4 to 6 half-lives) have been used as the standard procedure for the vast majority of drugs. The procedure of trough sampling immediately prior to the next dose has been chosen for practicality. Deviations from the correct sampling time immediately prior to the next dose are less critical for trough samples than during other phases after dose application, since the concentration time curve is relatively flat towards the end of the dosing interval (terminal ß-elimination phase).

Therapeutic ranges are determined in clinical studies correlating these trough concentrations with clinical outcomes. A frequent problem is, that blood sampling at different time points throughout the dosing interval leads to concentrations that may be misinterpreted as conferring an enhanced risk for adverse drug reactions when in reality true trough levels would be lower and no benchmark (therapeutic range) is available for such mistimed samples. As explained below, the expected trough concentration can and should then be computed. 
- Table 2 Inhibitors of CYP enzymes involved in drug metabolism.

\begin{tabular}{|c|c|c|}
\hline Inhibiting drugs & Inhibited enzymes & References \\
\hline Amiodarone & CYP2C9, CYP2D6, CYP3A4 & {$[790]$} \\
\hline Amprenavir & CYP3A4 & {$[1313]$} \\
\hline Aprepitant & CYP3A4 & [749] \\
\hline Atazanavir & CYP3A4 & {$[1266]$} \\
\hline Boceprevir & CYP3A4 & {$[407]$} \\
\hline Bupropion & CYP2D6 & {$[663]$} \\
\hline Cimetidine & CYP1A2, CYP2D6, CYP3A4 & [769] \\
\hline Ciprofloxacin & CYP1A2, CYP3A4 & {$[76]$} \\
\hline Clarithromycin & CYP3A4 & [969] \\
\hline Clomethiazole & CYP2E1 & [296] \\
\hline Clopidogrel & CYP2B6 & [996] \\
\hline Crizotinib & CYP3A4 & {$[761]$} \\
\hline Diltiazem & CYP3A4 & {$[1154]$} \\
\hline Disulfiram & CYP2E1 & [619] \\
\hline Duloxetine & CYP2D6 & {$[1096]$} \\
\hline Enoxacin & CYP1A2 & {$[1112]$} \\
\hline Erythromycin & CYP3A4 & {$[883]$} \\
\hline Esomeprazole & CYP2C19 & [879] \\
\hline Felbamate & CYP2C19 & {$[981]$} \\
\hline Fluconazole & CYP2C9, CYP3A4 & {$[860]$} \\
\hline Fluoxetine and norfluoxetine & CYP2D6, CYP2C19, CYP3A4 & {$[572]$} \\
\hline Fluvoxamine & CYP1A2, CYP2C8, CYP2C9, CYP2C19, CYP3A4 & {$[572]$} \\
\hline Fosamprenavir & CYP3A4 & [1313] \\
\hline Gemfibrocil & CYP2C8 & [59] \\
\hline Grapefruit juice & CYP3A4 & [1128] \\
\hline Indinavir & CYP3A4 & {$[1270]$} \\
\hline Isoniazid & CYP1A2, CYP2A6, CYP2C9, CYP2C19, CYP3A4, MAO & [859] \\
\hline Itraconazol & CYP3A4 & [1173] \\
\hline Ketoconazol & CYP3A4 & [293] \\
\hline Levomepromazine & CYP2D6 & {$[1258]$} \\
\hline Melperone & CYP2D6 & {$[502]$} \\
\hline Metoclopramide & CYP2D6 & {$[724]$} \\
\hline Miconazol & CYP2C9, CYP3A4 & {$[860]$} \\
\hline Moclobemide & CYP2C19, CYP2D6, MAO-A & {$[215,423,485]$} \\
\hline Nelfinavir & CYP3A4 & [629] \\
\hline Norfloxacine & CYP1A2 & [385] \\
\hline Omeprazole & CYP2C19 & {$[1282]$} \\
\hline Paroxetine & CYP2D6 & [572] \\
\hline Perazine & CYP1A2 & {$[360,1317]$} \\
\hline Phenylpropanolamine & CYP1A2 & {$[182]$} \\
\hline Posaconazole & CYP3A4 & {$[667]$} \\
\hline Propafenon & CYP1A2, CYP2D6 & {$[804]$} \\
\hline Quinidine & CYP2D6 & {$[142]$} \\
\hline Ritonavir & CYP2D6, CYP3A4 & {$[72,629,1270]$} \\
\hline Saquinavir & CYP3A4 & {$[72]$} \\
\hline Telaprevir & CYP3A4 & [394] \\
\hline Telithromycine & CYP3A4 & {$[601]$} \\
\hline Ticlopidine & CYP2B6, CYP2C19 & {$[996]$} \\
\hline Tranylcypromine & CYP2A6, MAO & [411] \\
\hline Valproic acid & CYP2C9 & {$[291,460]$} \\
\hline Verapamil & CYP3A4 & [692] \\
\hline Voriconazol & CYP2B6, CYP2C9, CYP2C19, CYP3A4 & [179] \\
\hline Zileuton & CYP1A2 & {$[426]$} \\
\hline
\end{tabular}


- Table 3 Inducers of enzymes and efflux transporters involved in drug metabolism and distribution.

\begin{tabular}{|c|c|c|c|}
\hline Inducing drugs & Induced enzymes or $\mathrm{ABC}$ transporters & Comments & References \\
\hline Bosentan & CYP3A4 & & {$[764]$} \\
\hline Carbamazepine & $\begin{array}{l}\text { CYP1A2, CYP2B6, CYP2C9, CYP3A4, P-gP } \\
\text { (ABCB1), UGT }\end{array}$ & $\begin{array}{l}\text { Increase of CYP3A4 activity within } 3 \text { weeks, induction } \\
\text { of its own metabolism }\end{array}$ & $\begin{array}{l}{[12,266,409,} \\
882,1122]\end{array}$ \\
\hline Efavirenz & CYP2B6, CYP3A4 & & {$[1004]$} \\
\hline Ethanol & CYP2E1 & Induction may lead to metabolic tolerance. & {$[590,708]$} \\
\hline Isoniazide & CYP2E1 & Initial inhibition and then induction of CYP2E1 & {$[1069,1343]$} \\
\hline Lamotrigine & UGT & & [266] \\
\hline Modafinil & CYP1A2, CYP2B6, CYP3A4 & & {$[1002]$} \\
\hline Oxybutynin & CYP3A4 & & [452] \\
\hline Phenobarbital & $\begin{array}{l}\text { CYP1A2, CYP2B6, CYP2C9, CYP2C19, } \\
\text { CYP3A4, UGT1A1 }\end{array}$ & & {$[742]$} \\
\hline Phenytoin & $\begin{array}{l}\text { CYP1A2, CYP2B6, CYP2C9, CYP2C19, } \\
\text { CYP3A4, UGT }\end{array}$ & & {$[60,266]$} \\
\hline Primidone & CYP2C9, CYP2C19, CYP3A4 & & [935] \\
\hline Rifabutin & CYP3A4 & Induction of own metabolism & [1349] \\
\hline Rifampicin & $\begin{array}{l}\text { CYP1A2, CYP2B6, CYP2C9, CYP2C19, } \\
\text { CYP3A4 }\end{array}$ & $\begin{array}{l}\text { After induction by rifampicin, CYP2C19 and CYP3A4 } \\
\text { activities remain elevated for } 4 \text { days after discontinua- } \\
\text { tion and return to baseline levels within } 8 \text { days. }\end{array}$ & {$[552,742]$} \\
\hline Ritonavir & CYP2C9, CYP3A4 (high dose), UGT & & [368] \\
\hline Smoke & CYP1A2 & $\begin{array}{l}\text { Maximal increase by } 10 \text { or more cigarettes per day, } \\
\text { decrease of CYP1A2 activity within } 3 \text { days after } \\
\text { smoking cessation }\end{array}$ & [342-343] \\
\hline St. John's wort & CYP3A4, CYP2C9, P-gP (ABCB1) & & {$[466]$} \\
\hline
\end{tabular}

- Fig. 2 shows that drug concentrations in blood depend on the choice of dosing. It is, therefore, mandatory to consider the dosing scheme used in clinical studies to derive therapeutic ranges. Clearly, the therapeutic ranges reported are only valid for the dosing scheme used in the respective study and cannot easily be transferred to other dosing schemes and application forms (iv, intramuscular depot etc.). Interpretation of TDM results becomes even more complicated when dosing schemes are used that distribute the daily dose in an unequal fashion, e. g., higher doses in the evening than during the day to achieve sedation during the night. Therefore, the dosing schemes relevant for therapeutic ranges are important for proper interpretation of the TDM result.

\subsubsection{Drug concentrations in brain and cerebrospinal fluid}

The pharmacologic activity of psychiatric and neurologic drugs depends on their availability at the target sites within the brain. The delivery of drugs from blood to brain takes place across brain capillary endothelial cells comprising the BBB [481]. The BBB controls the brain environment by efficiently restricting the exchange of solutes, e. g., by hindering the influx of potentially harmful xenobiotics including many drugs. The permeability of the BBB for a particular molecule defines the rate at which a drug enters brain interstitial fluid (ISF) from where the molecules will then be further distributed to and equilibrated within the brain cells [481]. Drug transportation from blood to cerebrospinal fluid (CSF) and vice versa takes place at the blood-CSF barrier (BCSFB) supplemented by an exchange between CSF and brain ISF. The CSF is an accessible sampling site for measuring drug concentrations of unbound drugs. Two systematic studies of 39 compounds by Fridén et al. [376] and 25 compounds by Kodaira et al. [652] demonstrated a good correlation between CSF and ISF drug concentrations for compounds that show a high permeability and little or no drug efflux via transporters. The role of CSF as a site for measuring unbound drug concentrations in brain, however, is still under discussion [481].

Drugs that are efficiently eliminated from the brain at the BBB are primarily P-gp substrates like risperidone, aripiprazole or venlafaxine [303, 639, 1217]. For these compounds, brain ISF concentrations are much lower than blood concentrations. When drugs are substrates of P-gp, the brain to blood concentration ratios vary widely for drugs with similar physicochemical properties. Animal studies found ratios ranging from 0.22 for risperidone [44] to 34 for fluphenazine [42]. Despite highly variable ratios of brain to blood concentrations of the different neuropsychiatric drugs, animal studies have shown that steady-state concentrations in blood correlate well with concentrations in brain, and much better than they correlate to the prescribed dosages. This has been shown, e.g., for tricyclic antidepressants [417], trazodone [287], or olanzapine [43]. In patients, it has been shown by magnetic resonance spectroscopy that brain concentrations of fluoxetine and norfluoxetine parallel concentrations in blood [607]. For carbamazepine and its epoxide, a linear relationship between brain and blood concentrations was found in patients undergoing brain surgery [821]. For neuropsychiatric medications, drug concentrations in blood can therefore be considered a valid marker of concentrations in brain. 


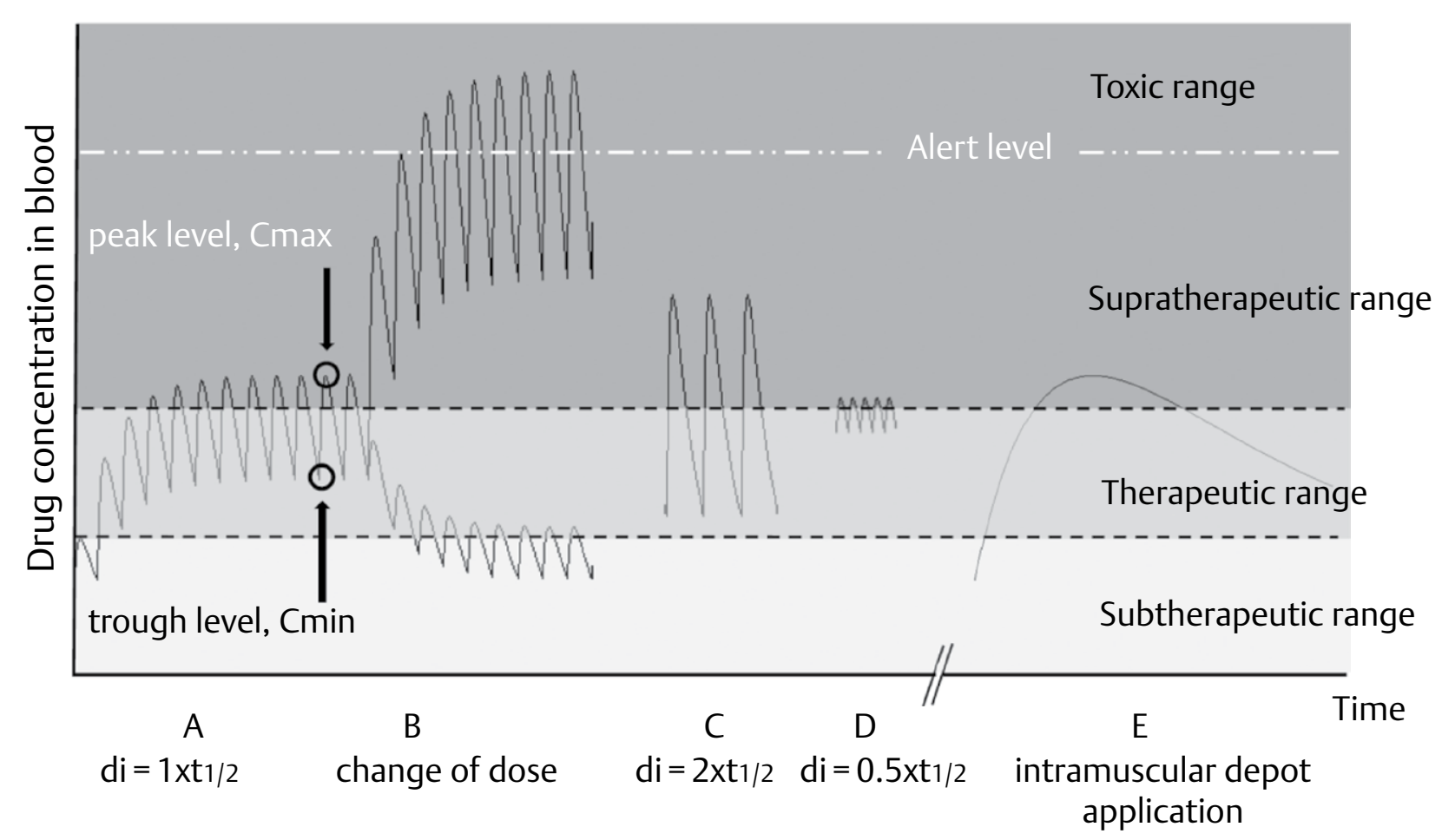

Fig. 2 Concentration time curve after oral or intramuscular depot medication. A: $94 \%$ of steady state (therapy with constant dose) is reached after four elimination half-lives (t1/2) of the drug. At steady-state, drug intake equals drug elimination over a defined time frame. Trough levels (Cmin) at steady state are usually quantified and recommended for TDM. The figure shows a hypothetical drug with a dosing interval (di) equal to its half-life $\left(d i=t_{1 / 2}\right)$, a situation found similar for many drugs (e. g., $t_{1 / 2}=12 \mathrm{~h}, \mathrm{di}=12 \mathrm{~h}$, curve A). Trough concentrations are right in the middle of the therapeutic range, i. e., on target, despite the fact that the drug's concentrations during the dosing interval sometimes exceed the therapeutic range. B: Modification of drug concentrations by doubling or halving the dose without change of the dosing interval. C: Doubling the dose interval $\left(\mathrm{di}=2 \times \mathrm{t}_{1 / 2}\right)$ and administering the entire daily dose once daily results in curve $\mathrm{C}$. The area under the blood concentration versus time curve (AUC) representing the total drug exposition is identical for curves $A$ and $C$, however, trough concentrations in curve $C$ ( $24 \mathrm{~h}$ after the daily dose) are significantly lower than in curve $\mathrm{A}(12 \mathrm{~h}$ after a half daily dose). High differences between trough and peak levels can be associated with tolerability problems during the phases of high drug concentrations. D: Curve D illustrates the intake of four equal doses per day, resulting in the same daily dose as for curves A to C. Again the AUC is identical to curves A and C but this time we observe higher trough concentrations. Using this application form, even low doses can be effective, since sufficient drug concentrations are available at the target structures. E: Intramuscular application of depot: Peak concentrations may be achieved after as early as 1 day or as late as 4 weeks depending on the formulation. Concentrations comparable to trough values after oral application can only be obtained immediately prior to the next application. Blood sampling during the elimination phase after full absorption (maximum) will result in higher values compared to trough sampling after oral application despite equal AUC. Please note the time scale for curve $E$ is different from curves $A$ to $D$.

Positron emission tomography (PET) enables analysis of central nervous receptor occupancy in vivo. PET studies have demonstrated that blood concentrations correlate well with the occupancy of target sites in the brain $[347,456,457,836,837,1213]$. Antipsychotic drugs exert most of their therapeutic actions by blockade of dopamine D2-like receptors [625]. Blockade of D2 receptors by antipsychotic drugs reduces the binding of radioactive PET ligands $[347,454,1213]$. Using this approach and by quantifying the displacement of dopamine receptor radioligands, it has been shown that receptor occupancy correlates better with concentrations of antipsychotic drugs in blood than with daily doses [525]. It is even possible to predict dopamine $D 2$ receptor occupancy based on the concentration of an antipsychotic drug in blood [1213]. Optimal clinical response was seen at $70-80 \%$ D2 receptor occupancy, and $80 \%$ D2 receptor occupancy was defined as the threshold for extrapyramidal symptoms $[347,868]$. PET was also used to charac- terize in vivo serotonin transporter (SERT or 5HTT) occupancy by serotonin reuptake inhibitors (SSRIs) $[46,69,800,801,864,1118$, $1165]$. Using a serotonin transporter radioligand, concentrations of citalopram, paroxetine, fluoxetine and sertraline in blood were shown to correlate well with serotonin transporter occupancy. At least $70 \%$ occupancy should be attained for optimal clinical outcome $[800,801]$. PET studies have thus brought about highly relevant information to determine therapeutically effective drug concentrations in blood for a considerable number of psychoactive drugs [456].

\subsection{Pharmacogenetic aspects}

The clinical importance of pharmacogenetic factors in the pharmacokinetics and pharmacodynamics of neuropsychiatric drugs is increasingly recognized [269, 341, 823, 1041]. As already mentioned above, drug-metabolizing enzymes, especially CYP isoen- 
zymes, exhibit genetic variability [1351-1353]. Extensive metabolizers (EM) are defined as wild-type with two active alleles. Poor metabolizers (PM) lack functional alleles. Intermediate metabolizers (IM) are either genetically heterozygous, carrying an active and an inactive allele or have one or two alleles with reduced activity. Ultrarapid metabolizers (UM) carry alleles with increased activity or multiplications of functional alleles [105]. Genetic polymorphisms of drug-metabolizing enzymes are clinically important. On the one hand, unexpected adverse drug reactions and toxicity may occur in PM due to increased blood concentrations. On the other hand, non-response may occur in UM due to subtherapeutic blood concentrations [272]. Prodrugs are activated by metabolism via CYP enzymes, e. g., codeine to morphine and tramadol to desmethyltramadol by CYP2D6 [547, 892]. In this situation, UMs are at risk for adverse drug reactions and PM patients will not be able to produce pharmacologically active metabolites. A new promising approach is the determination of mRNA encoding CYP1A2, CYP2C9 and CYP2C19 in leukocytes, mRNA levels were found to correlate well with hepatic CYP activities as shown by parallel probe drug phenotyping of CYP enzymes [1182].

Historically, the metabolizer status was determined with probe drugs such as caffeine for CYP1A2, omeprazole for CYP2C19, metoprolol or dextromethorphan for CYP2D6, or midazolam for CYP3A4/5 [722, 1170]. These phenotyping tests measure the metabolic situation of the patient at the moment of the test and allow detection of metabolic changes. They can thus be used to study the influence of environmental factors such as smoking or comedications on CYP activities [342, 343, 1098, 1357]. Over the last years, CYP genotyping has become more and more available. The clear advantage of genotyping is that it represents a "trait marker" and that its result is not influenced by environmental factors. It can be carried out in any situation and its result has a lifetime value. However, despite the fact that functional significance of the genetic variations for CYP enzymes is very well characterized [389], there is still appreciable variability caused by rare genetic variants which allows a probable prediction of the individual enzyme activity by genetic analyses focusing on the common variants only [774].

Other metabolizing enzyme systems such as UDP glucuronosyltransferases (UGT) also display genetic polymorphisms [245, 268], but their clinical relevance in pharmacotherapy and for dose adjustments is less well characterized than for CYP polymorphisms [1144].

With regard to $A B C B 1$ transporters and the functional role of its gene product P-gp for drug distribution in the body, the $A B C B 1$ genotype has been suggested to affect antidepressant and antipsychotic drug response. Patients may respond differently to P-gP substrate antidepressants and ABCB1 genotyping can be useful for improving antidepressant treatment outcome. Meanwhile, over 30 studies have investigated whether genetic variants within $A B C B 1$ predict clinical efficacy and/or tolerability of antidepressants in humans. In particular, minor allele carriers of the single nucleotide polymorphisms (SNPs) rs2032583 and rs2235040 were repeatedly found to be more susceptible to the effects of antidepressants than major allele carriers [146, 147, 263, 978, 1001, 1043, 1217]. Several other studies, however, did not observe better response rates or more adverse drug reactions among minor allele carriers than non-carriers $[111,301,927,1051]$. A pilot clinical trial with different doses of antidepressants that were P-gp substrates showed superior efficacy in carriers of the minor allele of rs2235083 at doses in the recommended dose range $[147,160]$. A dose increase strategy for the carriers of the major allele proved not effective. However, other strategies as switching to an antidepressant that is not substrate of the P-gp transporter have not yet been evaluated. Larger studies are therefore necessary before coming to a final conclusion as to the relevance and practical consequences of $A B C B 1$ genotypic variation.

In addition to the pharmacokinetic aspects reviewed above, there is increasing evidence for genetic factors driving pharmacodynamic processes such as interactions of drugs with receptors, enzymes, transporters, carrier proteins, structural proteins or ion channels to be crucially involved in mediating treatment response in mental disorders. In affective disorders, the serotonin transporter gene (5HTT; SLC6A4) is the most widely studied gene in this context. Results, however, have been inconclusive so far $[610,1071,1181]$. Applying a hypothesis-free approach, genomewide association studies (GWAS) have been conducted in the STAR * D, the Munich Antidepressant Response Signature (MARS) and the Genome-based Therapeutic Drugs for Depression (GENDEP) samples. These studies, however, failed to discern genomewide significant markers of antidepressant treatment response $[553,680]$. Response to lithium has also been investigated in the largest meta-analysis so far in a cohort of more than 2,500 patients from 22 research centers worldwide. While results may provide the basis for a better understanding of lithium mechanisms, they are not relevant as yet for clinical decision making [541, 679, 789, 1059]. In psychotic disorders, variation in the dopamine receptor genes DRD2, DRD3 and DRD4 have extensively been investigated regarding antipsychotic treatment response; these studies, however, did not yield robustly replicable results (for review see [143]). In alcohol dependent patients, recent meta-analytic data support a considerable role of the functional $\mathrm{A} 118 \mathrm{G}$ polymorphism of the $\mu$ opioid receptor gene (OPRM1) via a differential response to naltrexone [192]. However, more research is needed to determine the clinical validity (e.g., sensitivity, specificity, positive/negative predictive value) and utility profiles for pharmacogenetic approaches based on OPRM 1 variation in the treatment of alcohol use disorders [508].

Pharmacogenetic analyses at the pharmacodynamic level revealed promising first results regarding the genetic underpinnings of relevant adverse drug reactions of psychoactive drugs. The human leukocyte antigen markers HLA-B * 1502 and HLA-A * 3101 were consistently reported to confer a higher risk to develop Stevens-Johnson syndrome under carbamazepine treatment in patients of Asian descent [354,1328]. Some pharmacogenetic tests have been piloted in a clinical context such as the PGxPredict: CLOZAPINE test designed to predict agranulocytosis risk based on HLADQB1 gene variation, which, however, has been stopped given a high specificity (98.4\%), but a low sensitivity (21.5\%) [143]. 5-HTR2C, melanocortin 4 receptor (MC4R), neuropeptide Y (NPY), cannabinoid receptor 1 (CNR1) and leptin gene variations have been shown to mediate antipsychotic-induced weight gain (for review see [447]). Well-replicated gene variations have been described in antipsychotic-induced dystonia/tardive dyskinesia: Variations in RGS2 (regulator of G-protein signaling 2), a gene which 
modulates dopamine receptor signal transduction $[429,430]$, as well as variations in the serotonin receptor genes HTR2C $[18,19,1067]$ and possibly also HTR2A $[694,1066]$. A variation in the serotonin receptor gene HTR1A (rs6295; C-1019G) has consistently been associated with the antipsychotic treatment response of negative symptoms in schizophrenia [822, 1168].

To overcome the limitations of previous studies, the following strategies have been proposed: Focusing on one specific pharmacologic class and concentrating on more narrowly defined phenotypes (e.g., the International SSRI Pharmacogenomics Consortium, ISPC [112]), including pharmacokinetic variables (i. e. blood levels $[965,1227])$ and environmental influences [649], completing genetic coverage by including structural variation (e. g., copy number variation, CNV [873]), analysing interactive effects of multiple risk genes ('epistasis', e. g., [770]) and including epigenetic variation $[300,798]$. Along these lines, large worldwide consortia are currently being established in an attempt to conduct large-scale pharmacogenetic studies applying state-of-the-art techniques such as genome-wide association studies and exome sequencing, e. g., the International Consortium on Lithium Genetics (ConLiGen) [1059].

\section{Drug Concentrations in Blood to Guide Neuropsychopharmacotherapy}

To guide neuropsychopharmacotherapy, TDM considers pharmacodynamic and pharmacokinetic aspects. It has to be checked (1) whether the drug concentration is within the therapeutic reference range so that therapeutic efficacy and acceptable tolerability can be expected and (2) whether the blood concentration fits to the prescribed dosage to find out if the medication is taken as prescribed and/or if pharmacokinetic abnormalities are present. It must therefore be discriminated between therapeutically effective and expected dose-related drug concentrations [470,471]. Moreover, determination of metabolite to parent compound ratios and probe drug phenotyping enable evaluation of the individual pharmacokinetic phenotype.

\subsection{The therapeutic reference range}

The law of mass action implies that pharmacologic effects are concentration related [50]. TDM is based on this assumption with respect to both, therapeutic improvement and adverse drug reactions. TDM also assumes that there is a concentration range of the drug in blood for maximal effectiveness and acceptable safety, the so-called "therapeutic reference range". Studies on relationships between drug concentration in blood and clinical improvement have supported this concept since the 1960s for lithium, tricyclic antidepressants and first-generation antipsychotic drugs. Systematic reviews and meta-analyses that were based on adequately designed studies demonstrated a significant relationship between clinical outcome and drug concentration in blood for nortriptyline, imipramine and desipramine, which are associated with a high probability of response [82]. For amitriptyline as a model compound, a meta-analysis of 45 studies has shown that various statistical approaches provided almost identical therapeutic reference ranges [1222, 1224]. For new antipsychotic drugs like aripiprazole [1115], olanzapine [933] or risperi- done [1336], relationships between drug concentration in blood and clinical effectiveness have been reported [729].

The therapeutic reference range is an essential target range for TDM guided pharmacotherapy. Its estimation requires determination of a lower and an upper limit of therapeutically effective and tolerable drug concentrations in blood. A generally accepted method to estimate these limits does not exist, and methodological restrictions such as placebo response or treatment resistance must be considered [ 50, 329, 958]. PET studies were most helpful to define these limits for antipsychotic and antidepressant drugs. The PET technique, however, is highly expensive and available only in few centers. Fixed dose studies are the most appropriate way to determine therapeutic reference ranges. Their determination, however, is actually not legally required for drug approval. We strongly advise that drug monitoring should be implemented into the development process of new drugs during the clinical research phase. To do this, established concepts of clinical trials (fixed dose studies) must be supplemented by measuring drug concentrations in blood.

For "therapeutic reference range", there are a lot of synonymous terms like "therapeutic window", "therapeutic range", "optimal plasma concentration”, "effective plasma concentration”, "target range", "target concentration", or "orienting therapeutic range", the term used in the first TDM consensus [82]. The AGNP TDM task force decided in 2011 to use the term "therapeutic reference range" following the convention of TDM guidelines published for antiepileptic drugs [912], and to use the term "drug concentration in blood" which includes plasma concentration, serum concentration or plasma level, serum level or blood level.

\section{Definition}

The "therapeutic reference ranges" reported in these guidelines ( $\triangleright$ Table 4 ) define ranges of drug concentrations in blood that specify a lower limit below which a drug induced therapeutic response is relatively unlikely to occur and an upper limit above which tolerability decreases or above which it is relatively unlikely that therapeutic improvement may be still enhanced. The therapeutic reference range is an orienting, population based range, which may not necessarily be applicable to all patients. Individual patients may show optimal therapeutic response under a drug concentration that differs from the therapeutic reference range. Ultimately, neuropsychopharmacotherapy can be best guided by identification of the patient's individual therapeutic concentration. The therapeutic reference ranges as recommended by the TDM group of the AGNP are given in $>$ Table 4.

Therapeutic reference ranges shown in $>$ Table 4 are evidencebased and derived from the literature by the structured review process described above. Therapeutic reference ranges that are based on randomized clinical trials were found for only 17 neuropsychiatric drugs in the literature. For most drugs, reference ranges were obtained from studies with therapeutically effective doses. The reference ranges listed in $>$ Table $\mathbf{4}$ are generally those for the primary indication. A number of drugs, however, are recommended for several indications. For example, antidepressant drugs are also used 


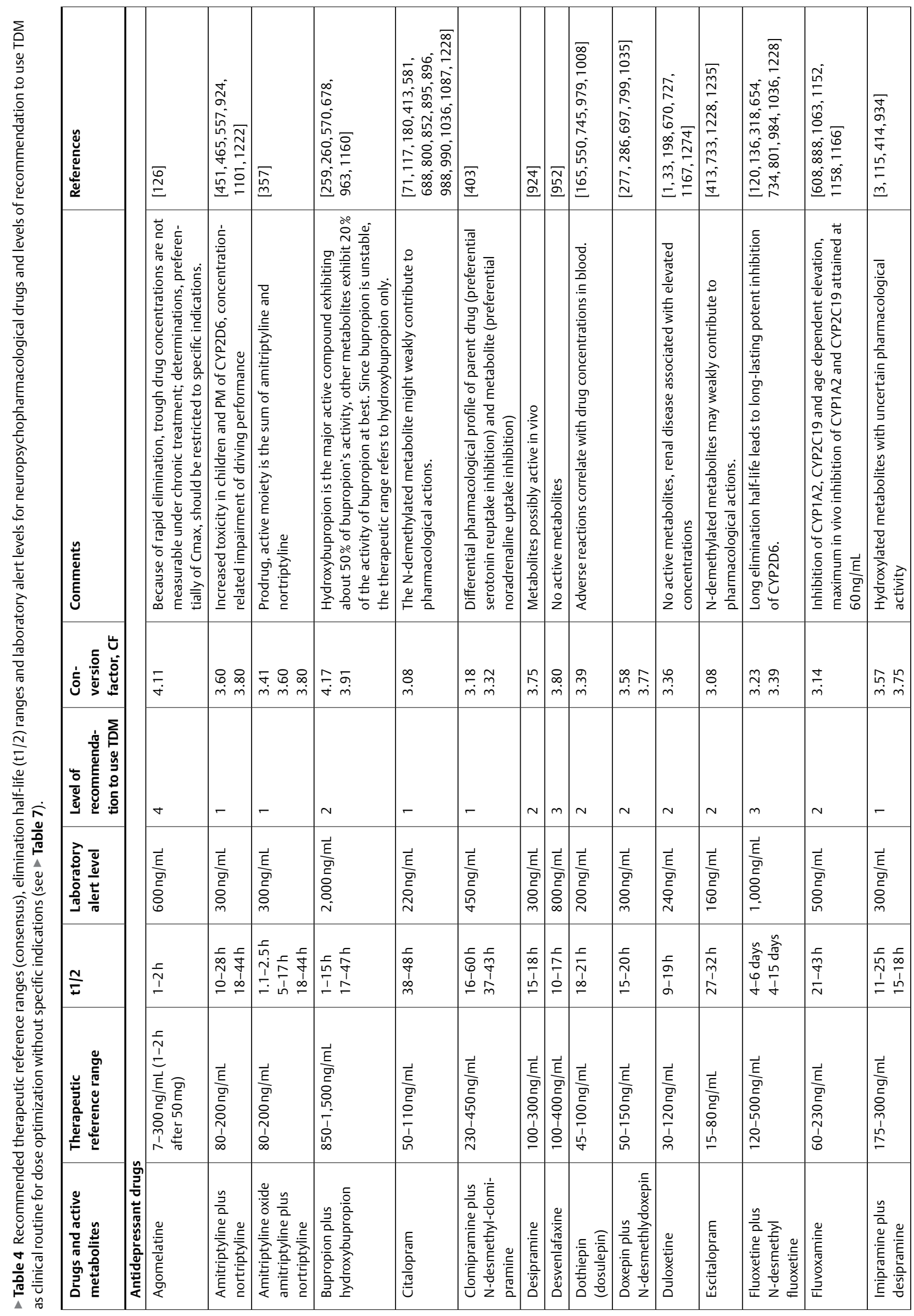




\begin{tabular}{|c|c|c|c|c|c|c|c|c|c|c|c|c|c|c|c|c|c|}
\hline 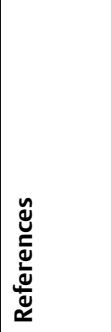 & 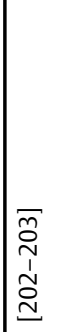 & 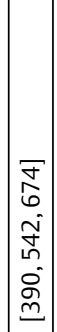 & \begin{tabular}{|c|} 
\\
\\
\\
0 \\
0 \\
0 \\
0 \\
0 \\
$\tilde{D}$ \\
0 \\
\end{tabular} & 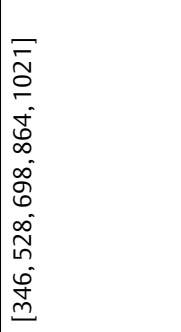 & 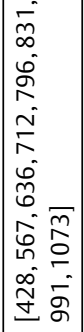 & 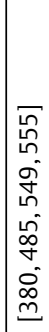 & 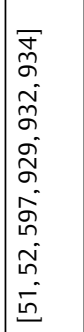 & 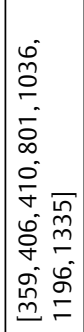 & \begin{tabular}{|c|} 
\\
\\
\\
$\overline{0}$ \\
$\infty$ \\
$\infty$ \\
0 \\
0 \\
$\infty$ \\
0 \\
\end{tabular} & 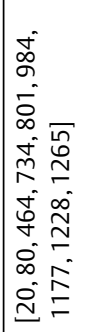 & $\begin{array}{l}\overleftarrow{\Xi} \\
\stackrel{\Xi}{\Xi}\end{array}$ & $\begin{array}{l}\infty \\
\text { 总 } \\
\text { 目 }\end{array}$ & 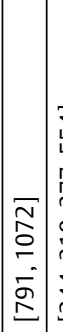 & 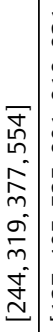 & 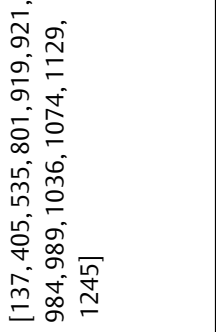 & 导 & 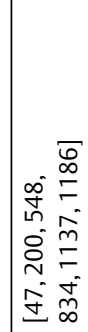 \\
\hline 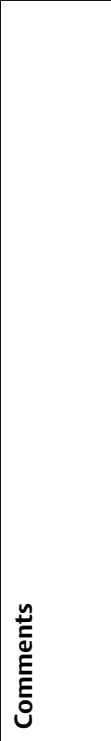 & 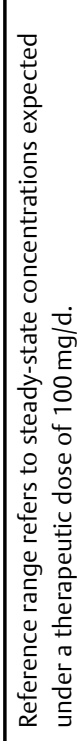 & 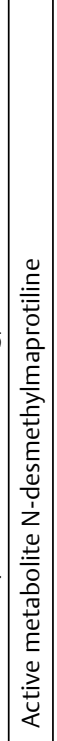 & & 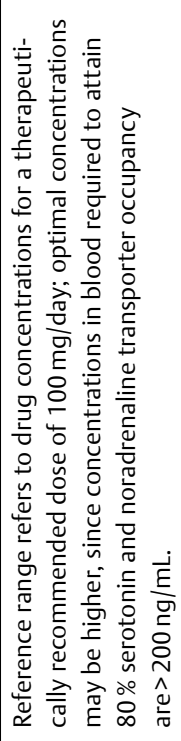 & 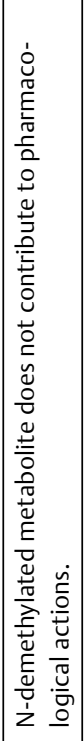 & 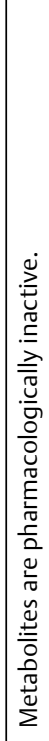 & 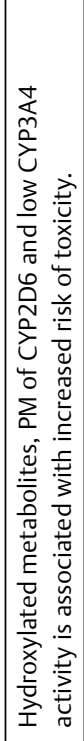 & 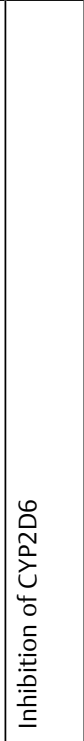 & & 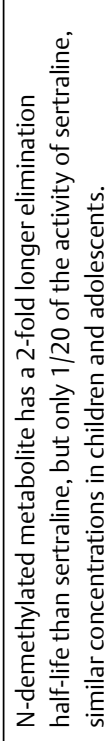 & & 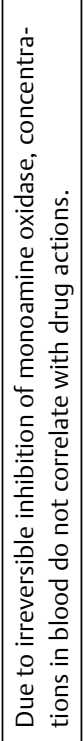 & & 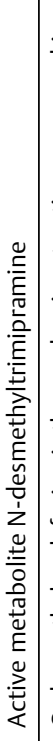 & 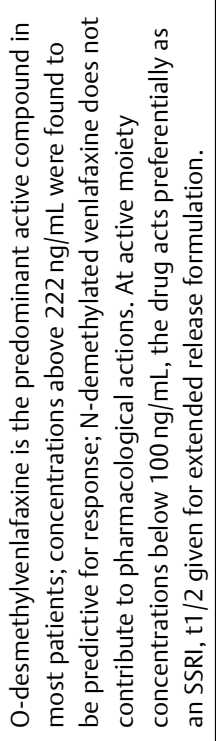 & 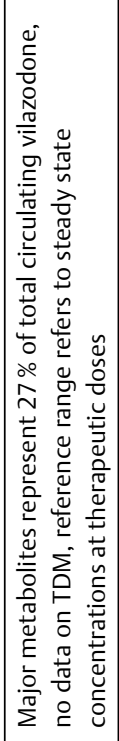 & 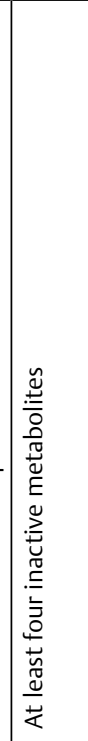 \\
\hline 空 & $\stackrel{\underset{N}{N}}{\stackrel{d}{*}}$ & $\mid \begin{array}{l}\dot{b} \\
\dot{p}\end{array}$ & $\left|\begin{array}{c}\infty \\
\\
m\end{array}\right|$ & $\underset{\sim}{\stackrel{d}{N}}$ & $\underset{\dot{m}}{\stackrel{N}{n}}$ & $\underset{m}{\stackrel{N}{N}}$ & $\begin{array}{l}\infty \\
\stackrel{\infty}{m}\end{array}$ & $\underset{\dot{m}}{\Delta}$ & $\frac{\sigma}{\dot{m}}$ & $\underset{j}{\tilde{m}}$ & $\begin{array}{l}\stackrel{D}{\infty} \\
\stackrel{i}{i}\end{array}$ & ᄃํ. & $\begin{array}{l}0 \\
\dot{i} \\
i\end{array}$ & $\begin{array}{l}q \\
\dot{m} \\
\end{array}$ & 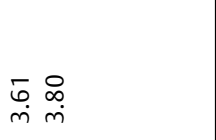 & 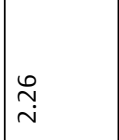 & $\stackrel{\stackrel{m}{m}}{m}$ \\
\hline 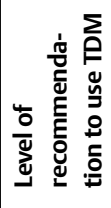 & $m$ & $\sim$ & $m$ & $\sim$ & N & $m$ & - & $m$ & $m$ & $\sim$ & $m$ & $\nabla$ & $\sim$ & $\sim$ & $\sim$ & $m$ & $\sim$ \\
\hline 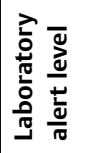 & 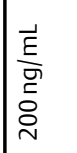 & $\left|\begin{array}{l}\vec{E} \\
\vec{E} \\
\vec{\vdots} \\
\grave{N}\end{array}\right|$ & 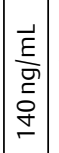 & $\begin{array}{l}\vec{E} \\
\frac{\vec{E}}{\sigma} \\
\bar{\delta} \\
\dot{D} \\
m\end{array}$ & 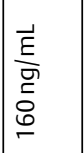 & 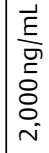 & $\begin{array}{l}\vec{E} \\
\frac{\vec{E}}{\sigma} \\
\bar{\Xi} \\
\bar{m}\end{array}$ & 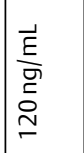 & $\mid \begin{array}{l}\vec{E} \\
\frac{\vec{E}}{\overrightarrow{5}} \\
\grave{\vdots}\end{array}$ & $\begin{array}{l}\vec{E} \\
\frac{\vec{E}}{g} \\
\bar{\Xi} \\
\dot{m}\end{array}$ & 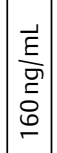 & $\begin{array}{l}\vec{E} \\
\frac{\vec{E}}{g} \\
\dot{\Xi} \\
\stackrel{口}{-}\end{array}$ & 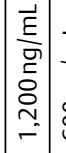 & $\begin{array}{l}\vec{E} \\
\vec{E} \\
\\
\vdots \\
\vdots \\
0\end{array}$ & $\begin{array}{l}\vec{E} \\
\frac{\vec{E}}{\sigma} \\
\bar{\Xi} \\
\infty\end{array}$ & $\begin{array}{l}\vec{E} \\
\frac{\vec{E}}{g} \\
\bar{\sigma} \\
\underline{I}\end{array}$ & $\mid$\begin{tabular}{|l}
$\vec{E}$ \\
$\frac{\vec{E}}{⿹}$ \\
$\bar{D}$ \\
$\infty$
\end{tabular} \\
\hline$\frac{N}{J}$ & \begin{tabular}{l}
\multicolumn{1}{c}{} \\
$\hat{b}$ \\
\end{tabular} & 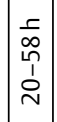 & 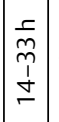 & \begin{tabular}{|l}
$\frac{r}{\infty}$ \\
$\infty$ \\
1 \\
$⺊$
\end{tabular} & $\begin{array}{l}\text { c } \\
\text { o } \\
\text { 1 } \\
\text { ì }\end{array}$ & $\frac{r}{i}$ & 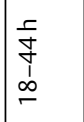 & $\begin{array}{l}\stackrel{5}{f} \\
\stackrel{f}{d} \\
\stackrel{1}{\sim}\end{array}$ & $\begin{array}{l}\mathfrak{r} \\
\mathfrak{N} \\
1 \\
\infty\end{array}$ & $\begin{array}{l}\frac{5}{0} \\
\stackrel{n}{1} \\
\stackrel{\sim}{N}\end{array}$ & $\mid \begin{array}{c}\frac{r}{\hat{H}} \\
\hat{\omega} \\
\dot{N}\end{array}$ & $\frac{r}{\mathfrak{m}}$ & 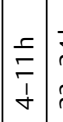 & 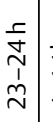 & 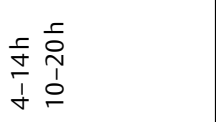 & $\begin{array}{l}\underset{n}{\sim} \\
\tilde{n} \\
\infty \\
-\infty\end{array}$ & \begin{tabular}{|l}
\multicolumn{1}{c}{} \\
0 \\
0 \\
1 \\
in
\end{tabular} \\
\hline 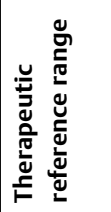 & 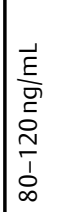 & 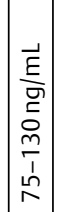 & 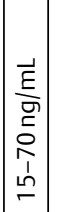 & 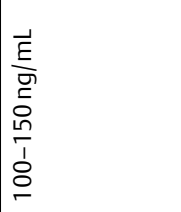 & 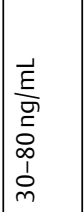 & 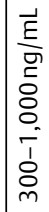 & 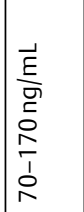 & 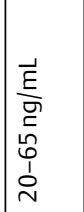 & 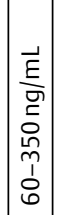 & 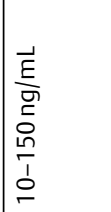 & 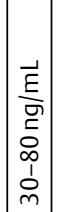 & 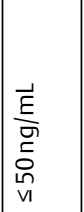 & 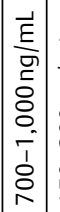 & 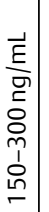 & 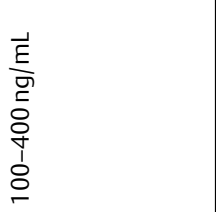 & 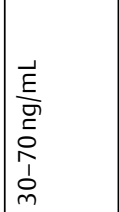 & 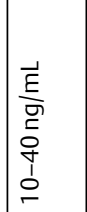 \\
\hline 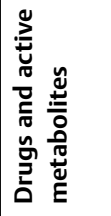 & 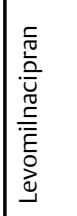 & 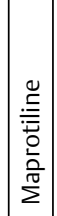 & 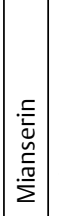 & 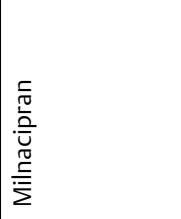 & 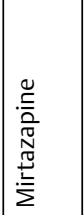 & 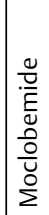 & 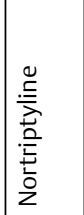 & 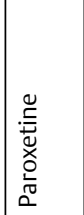 & 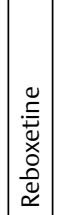 & 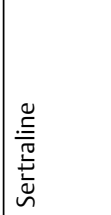 & 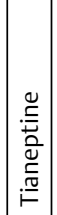 & 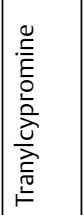 & 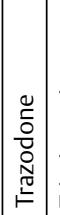 & 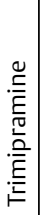 & 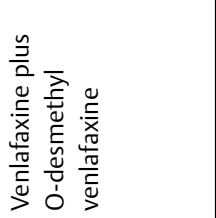 & \begin{tabular}{|l} 
\\
$\frac{\tilde{\sigma}}{0}$ \\
$\frac{0}{0}$ \\
$\frac{\pi}{5}$ \\
$\frac{\pi}{5}$
\end{tabular} & 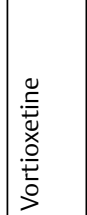 \\
\hline
\end{tabular}




\begin{tabular}{|c|c|c|c|c|c|c|c|c|c|c|c|c|c|c|c|c|c|c|c|c|c|}
\hline 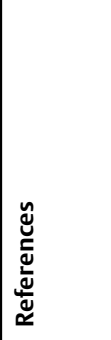 & & 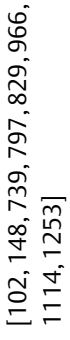 & 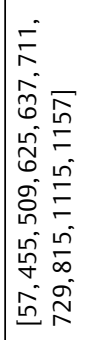 & 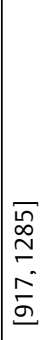 & \begin{tabular}{|l}
$\infty$ \\
0 \\
0 \\
0 \\
0 \\
$\infty$ \\
0 \\
0 \\
0 \\
0 \\
0
\end{tabular} & $\underset{\widetilde{N}}{\stackrel{\bar{\Xi}}{\simeq}}$ & $\begin{array}{l}\hat{n} \\
m \\
\tilde{n} \\
\tilde{\sigma} \\
\tilde{\sigma} \\
\infty \\
\infty \\
0 \\
\Xi\end{array}$ & 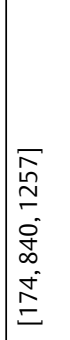 & 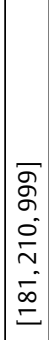 & $\begin{array}{l} \\
\delta \\
\delta \\
0 \\
0 \\
0 \\
0 \\
0 \\
0\end{array}$ & 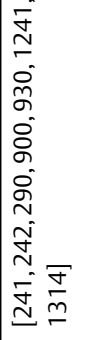 & $\begin{array}{l}\frac{n}{n} \\
0 \\
0 \\
\tilde{n} \\
0 \\
o \\
\infty \\
\infty \\
0 \\
\tilde{\omega} \\
\underline{0}\end{array}$ & 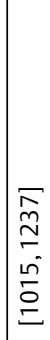 & E & 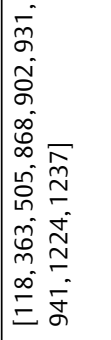 & 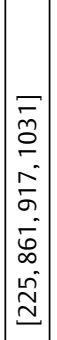 & 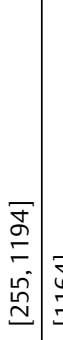 & 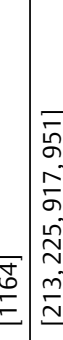 & 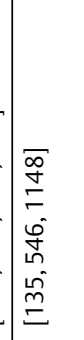 & 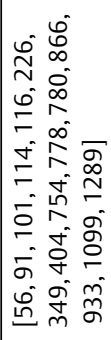 & 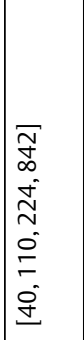 \\
\hline 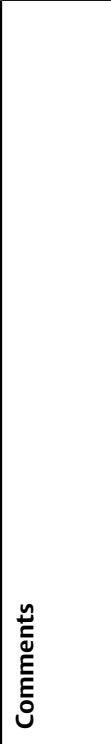 & & 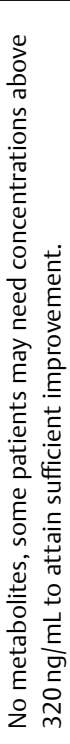 & 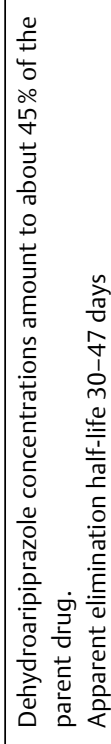 & & 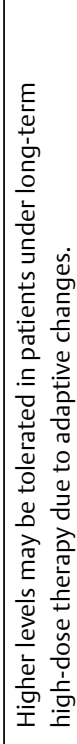 & 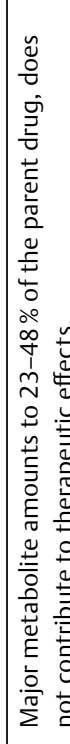 & & 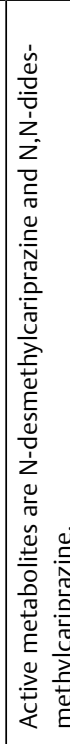 & & & 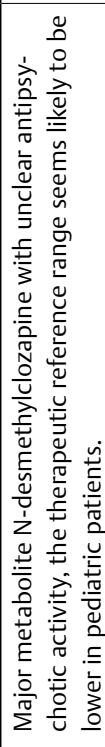 & 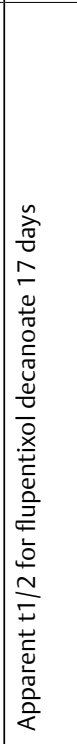 & 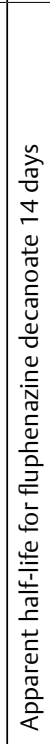 & & 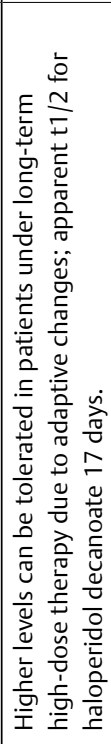 & & & 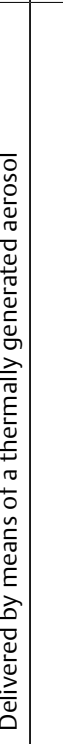 & 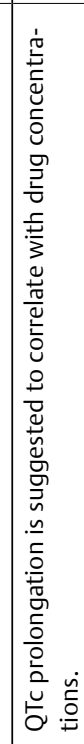 & 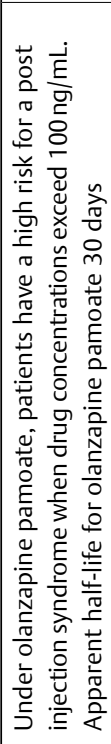 & 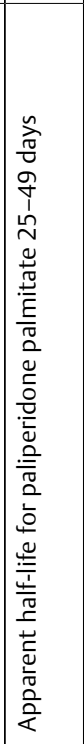 \\
\hline (5 & & $\underset{i}{i}$ & 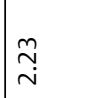 & 㝕 & 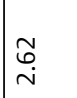 & & 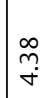 & $\stackrel{+}{\stackrel{m}{N}}$ & $\frac{\nabla}{\stackrel{J}{m}}$ & $\begin{array}{l}\tilde{O} \\
\dot{m}\end{array}$ & $\mid \begin{array}{l}\mathscr{L} \\
\dot{m}\end{array}$ & 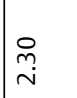 & 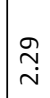 & $\frac{O}{i}$ & ن. & 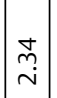 & 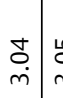 & 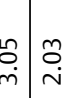 & 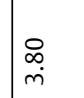 & 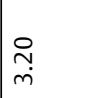 & $\stackrel{\stackrel{n}{N}}{\stackrel{N}{N}}$ \\
\hline 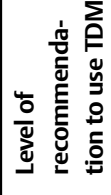 & & - & $\sim$ & t & $m$ & $m$ & $\sim$ & $m$ & $\sim$ & $m$ & - & $\sim$ & - & $m$ & - & $m$ & $\mathrm{~m} / \mathrm{n}$ & $m \mid m$ & $m$ & - & $\sim$ \\
\hline 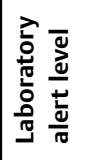 & & $\begin{array}{l}\vec{E} \\
\frac{\vec{E}}{\bar{G}} \\
\text { 売 }\end{array}$ & 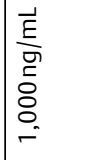 & \begin{tabular}{|l}
$\overrightarrow{\underline{E}}$ \\
$\frac{\vec{g}}{\mathrm{~g}}$ \\
$\underline{\underline{\sigma}}$
\end{tabular} & 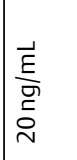 & 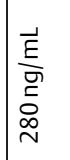 & \begin{tabular}{|l}
$\vec{E}$ \\
$\underline{G}$ \\
$\bar{\Xi}$ \\
$\bar{m}$
\end{tabular} & \begin{tabular}{|l}
$\overrightarrow{\underline{E}}$ \\
$\bar{\sigma}$ \\
$\bar{\sigma}$ \\
$\sigma$
\end{tabular} & $\left|\begin{array}{|c|}\vec{E} \\
\vec{g} \\
\bar{\Xi} \\
\dot{D} \\
0\end{array}\right|$ & $\left|\begin{array}{c}\vec{E} \\
\bar{g} \\
\underline{\Xi} \\
\grave{q}\end{array}\right|$ & 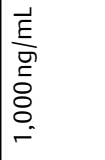 & $\begin{array}{l}\overrightarrow{\underline{E}} \\
\underline{\underline{V}} \\
\stackrel{\underline{n}}{\underline{n}}\end{array}$ & $\begin{array}{l}\overrightarrow{\underline{E}} \\
\stackrel{\vec{g}}{\underline{\underline{n}}} \\
\underline{\underline{n}}\end{array}$ & $\mid$\begin{tabular}{|l}
$\vec{E}$ \\
$\vec{G}$ \\
$\vec{g}$ \\
$\dot{\vec{\sigma}}$ \\
\end{tabular} & $\begin{array}{l}\overrightarrow{\underline{E}} \\
\underline{\sigma} \\
\stackrel{\underline{n}}{L}\end{array}$ & $\mid$\begin{tabular}{|l}
$\vec{E}$ \\
$\vec{\Xi}$ \\
$\stackrel{\vec{N}}{N}$
\end{tabular} & $\begin{array}{l}\vec{E} \\
\overline{0} \\
\vdots \\
\overline{\tilde{D}} \\
m\end{array}$ & 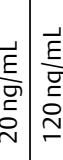 & $\begin{array}{l}\vec{E} \\
\text { 至 } \\
\text { 高 }\end{array}$ & 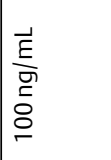 & 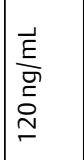 \\
\hline$\frac{N}{=}$ & & $\begin{array}{l}\stackrel{c}{\grave{I}} \\
\stackrel{1}{\simeq}\end{array}$ & \begin{tabular}{l}
\multicolumn{1}{c}{} \\
0 \\
0 \\
1 \\
0 \\
0
\end{tabular} & $\begin{array}{l}\frac{5}{\sigma} \\
\tilde{m} \\
m \\
\underline{r}\end{array}$ & $\mid \begin{array}{l}5 \\
\infty \\
\dot{b} \\
\dot{f}\end{array}$ & $\frac{5}{\sigma}$ & $\begin{array}{l}\frac{1}{0} \\
\hat{0} \\
\hat{1} \\
\stackrel{1}{N}\end{array}$ & 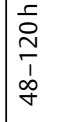 & \begin{tabular}{l}
\multicolumn{1}{c}{} \\
0 \\
$m$ \\
\\
$r$ \\
\end{tabular} & $\begin{array}{l}\mathfrak{r} \\
\mathfrak{N} \\
1 \\
\infty \\
\end{array}$ & $\begin{array}{l}\frac{5}{0} \\
\stackrel{1}{1} \\
\stackrel{2}{\simeq}\end{array}$ & $\begin{array}{l}\text { co } \\
\text { ơ } \\
\text { ò } \\
\text { N }\end{array}$ & $\frac{5}{6}$ & 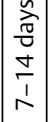 & $\begin{array}{l}\frac{r}{0} \\
\stackrel{1}{1} \\
\stackrel{1}{\sim}\end{array}$ & 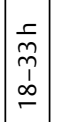 & 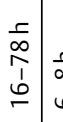 & 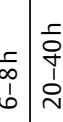 & $\begin{array}{l}5 \\
0 \\
1 \\
t\end{array}$ & $\begin{array}{l}\text { r } \\
\text { oे } \\
0 \\
1 \\
\text { m }\end{array}$ & $\begin{array}{l}\frac{1}{\tilde{N}} \\
\stackrel{1}{\sim} \\
\stackrel{1}{\sim}\end{array}$ \\
\hline 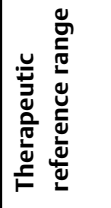 & & 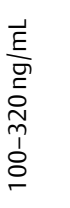 & 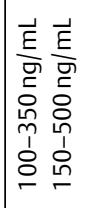 & 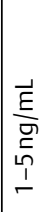 & 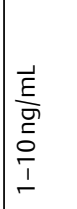 & 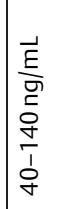 & 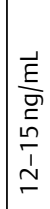 & 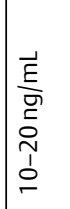 & 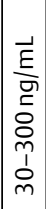 & 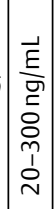 & $\begin{array}{l}\vec{E} \\
\frac{E}{5} \\
\underline{\Xi} \\
\delta \\
0 \\
1 \\
0 \\
0 \\
m\end{array}$ & 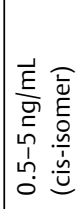 & 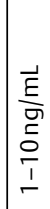 & 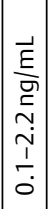 & 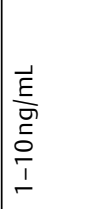 & 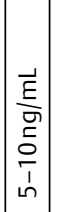 & \begin{tabular}{l|l}
\multicolumn{1}{c}{} & \\
& \\
$\vdots$ & \\
0 & \\
1 & \\
1 & \\
$m$ &
\end{tabular} & 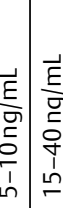 & 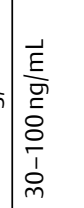 & 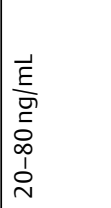 & 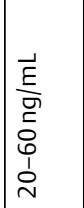 \\
\hline 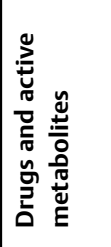 & 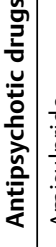 & 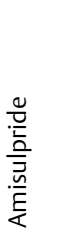 & 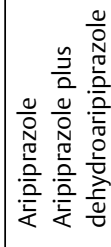 & 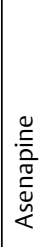 & 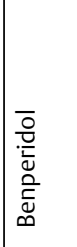 & 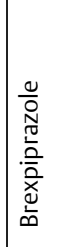 & 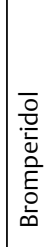 & 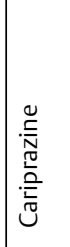 & 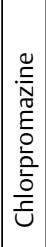 & 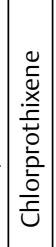 & 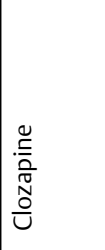 & 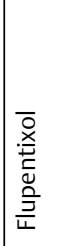 & 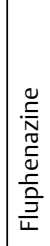 & $\frac{\bar{u}}{\underline{\underline{L}}}$ & $\begin{array}{l}\frac{0}{\frac{0}{0}} \\
\frac{0}{0} \\
\frac{0}{\frac{0}{T}} \\
\frac{10}{1}\end{array}$ & 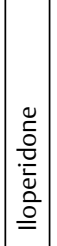 & 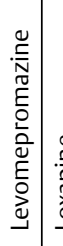 & 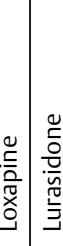 & 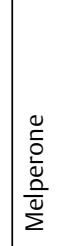 & 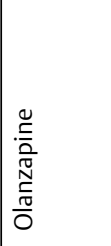 & 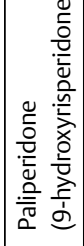 \\
\hline
\end{tabular}




\begin{tabular}{|c|c|c|c|c|c|c|c|c|c|c|c|c|c|c|c|c|c|c|}
\hline & $\begin{array}{l}\overline{\bar{n}} \\
\check{c}\end{array}$ & 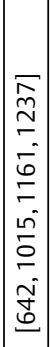 & 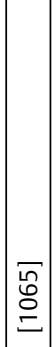 & $\mid \begin{array}{l}\bar{J} \\
\mathcal{G} \\
\bar{f} \\
\underline{m}\end{array}$ & 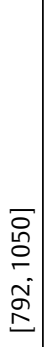 & 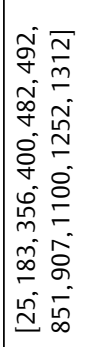 & 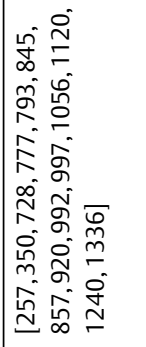 & 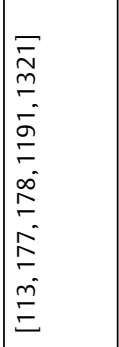 & 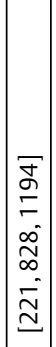 & 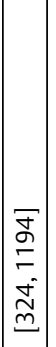 & 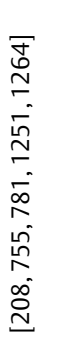 & 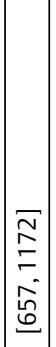 & 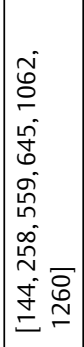 & & $\begin{array}{l}\tilde{N} \\
\tilde{N} \\
\tilde{\tilde{D}} \\
\underline{\underline{0}}\end{array}$ & 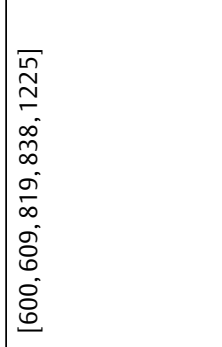 & \begin{tabular}{|l}
1 \\
0 \\
0 \\
0 \\
0 \\
0 \\
0 \\
0
\end{tabular} & 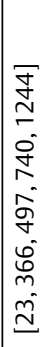 \\
\hline & & 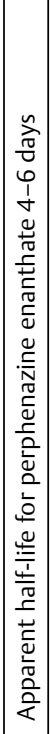 & & & 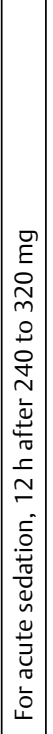 & 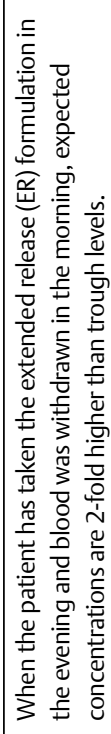 & 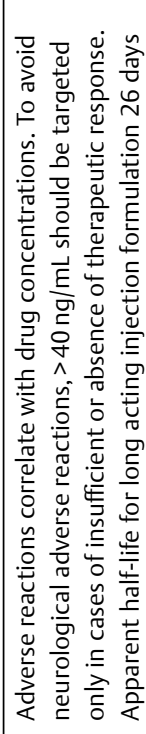 & 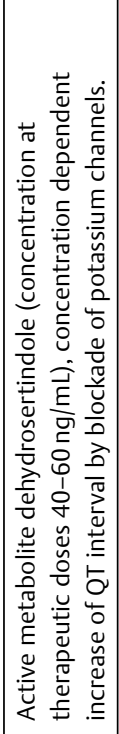 & 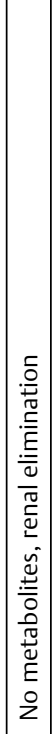 & 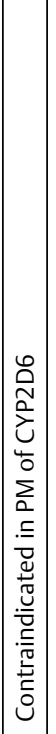 & 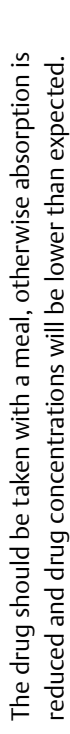 & & 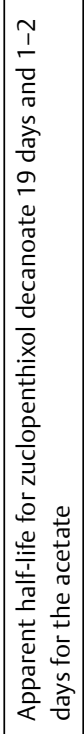 & & 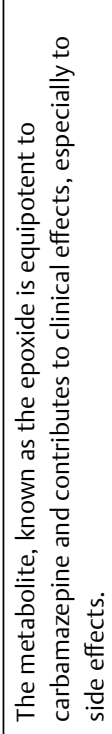 & 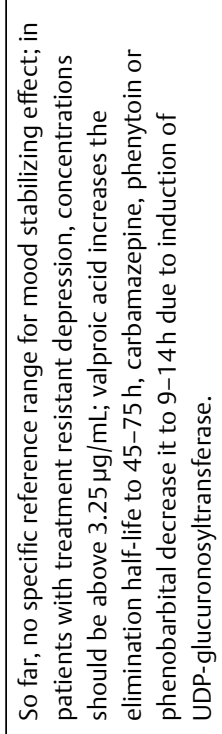 & 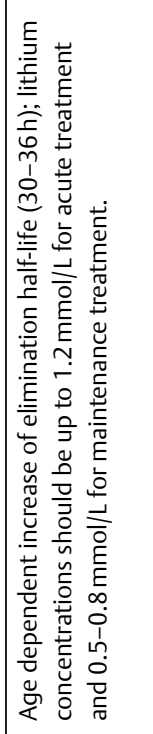 & 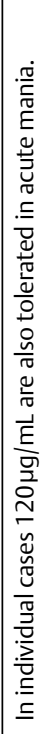 \\
\hline 它空 & $\mid \begin{array}{c}n \\
\stackrel{n}{\mathrm{v}} \\
\end{array}$ & $\mid \begin{array}{l}\stackrel{\infty}{\sim} \\
\mathrm{N}\end{array}$ & $\stackrel{\vec{\nu}}{\stackrel{i}{i}}$ & $\begin{array}{l}\stackrel{\leftrightarrow}{\circ} \\
\stackrel{i}{i}\end{array}$ & 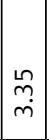 & $\stackrel{\bar{\sigma}}{\stackrel{m}{\sim}} \stackrel{m}{m}$ & 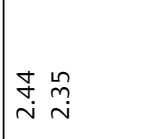 & 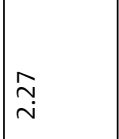 & $\mid \begin{array}{c}\tilde{g} \\
\dot{v}\end{array}$ & $\begin{array}{l}\stackrel{P}{i} \\
\dot{\sim}\end{array}$ & $\stackrel{\stackrel{\leftrightarrow}{\sim}}{\sim}$ & $\begin{array}{l}\bar{\sigma} \\
\dot{m}\end{array}$ & 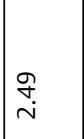 & & 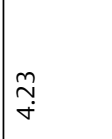 & $\begin{array}{l}8 \\
\text { m }\end{array}$ & 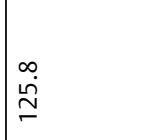 & శ్ \\
\hline 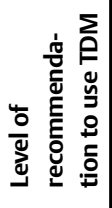 & - & - & $m$ & $m$ & $\nabla$ & $\sim$ & $\sim$ & $\sim$ & $\sim$ & - & $\sim$ & $m$ & $m$ & & - & $\sim$ & - & 7 \\
\hline 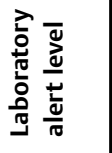 & 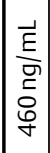 & $\begin{array}{c}\vec{E} \\
\vec{E} \\
\end{array}$ & 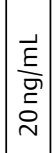 & 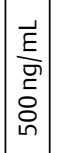 & 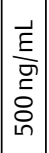 & 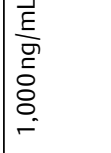 & $\begin{array}{l}\vec{E} \\
\text { Ẽ } \\
\underline{\Xi} \\
\stackrel{D}{I}\end{array}$ & 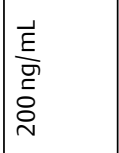 & 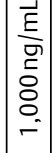 & 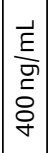 & $\begin{array}{l}\vec{E} \\
\frac{\vec{g}}{\sigma} \\
\bar{\sigma} \\
\dot{g}\end{array}$ & 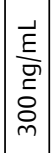 & 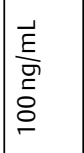 & & $\begin{array}{l}\frac{\vec{E}}{\sigma} \\
\frac{9}{2} \\
\stackrel{\nu}{N}\end{array}$ & 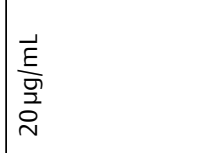 & 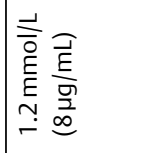 & \\
\hline$\frac{N}{I}$ & \begin{tabular}{|c|}
5 \\
6 \\
1 \\
$\infty$ \\
\end{tabular} & $\begin{array}{l}\mathbf{r} \\
\mathfrak{N} \\
1 \\
\infty \\
\end{array}$ & \begin{tabular}{|l|}
$\frac{\tilde{m}}{\tilde{m}}$ \\
$\tilde{y}$ \\
$\tilde{N}$
\end{tabular} & 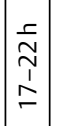 & $\left|\begin{array}{c}\frac{c}{m} \\
\grave{\sim}\end{array}\right|$ & 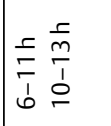 & 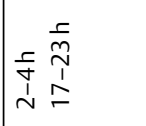 & 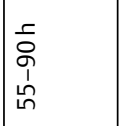 & $\mid \begin{array}{c}f \\
f \\
\vdots \\
\infty\end{array}$ & $\mid \begin{array}{l}\frac{1}{2} \\
\text { m }\end{array}$ & 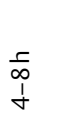 & 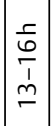 & 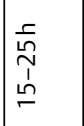 & & 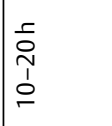 & $\begin{array}{l}\frac{f}{d} \\
\frac{f}{1} \\
\frac{1}{\square}\end{array}$ & \begin{tabular}{l}
\multicolumn{1}{c}{} \\
$\overline{0}$ \\
$\tilde{n}$ \\
$\dot{t}$ \\
\end{tabular} & $=$ \\
\hline 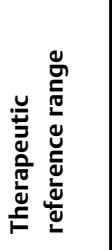 & 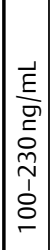 & $\mid \begin{array}{c}\vec{E} \\
\underline{E} \\
0 \\
\vec{c} \\
\dot{i} \\
\underline{b} \\
0 \\
0\end{array}$ & 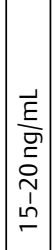 & 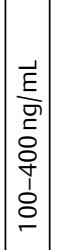 & 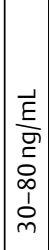 & 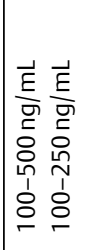 & $\begin{array}{l}\vec{E} \\
\text { ज्ञ } \\
\underline{E} \\
0 \\
0 \\
1 \\
D \\
N\end{array}$ & 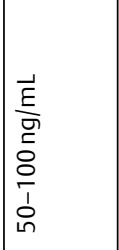 & 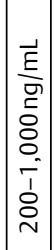 & 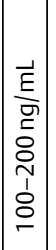 & 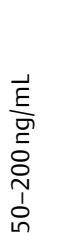 & 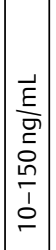 & 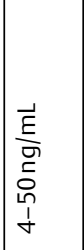 & & \begin{tabular}{|l}
$\bar{E}$ \\
$\frac{\bar{g}}{5}$ \\
$\frac{0}{1}$ \\
$\dot{\gamma}$
\end{tabular} & $\mid \begin{array}{l}\vec{E} \\
\frac{E}{5} \\
\underline{9} \\
0 \\
\underline{1} \\
-\end{array}$ & 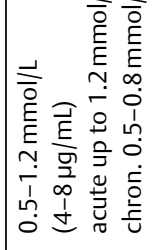 & \begin{tabular}{|l}
$\frac{\vec{E}}{\mathrm{~g}}$ \\
$\frac{\mathrm{g}}{\mathrm{g}}$ \\
$\mathrm{g}$
\end{tabular} \\
\hline 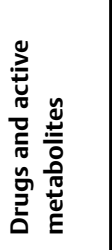 & $\stackrel{\mathscr{I}}{.}$ & 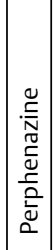 & 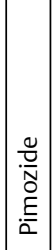 & 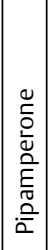 & & 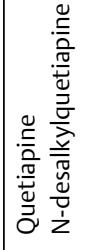 & 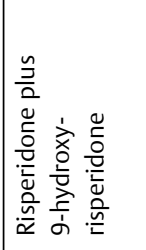 & 吾 & 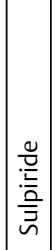 & 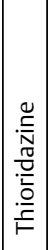 & 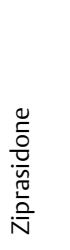 & 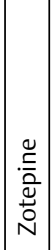 & 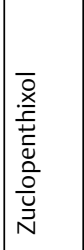 & 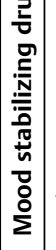 & 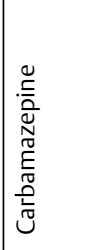 & 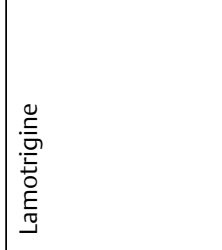 & $\mid \begin{array}{l}\underline{\underline{\underline{E}}} \\
\underline{\underline{\underline{E}}}\end{array}$ & \\
\hline
\end{tabular}




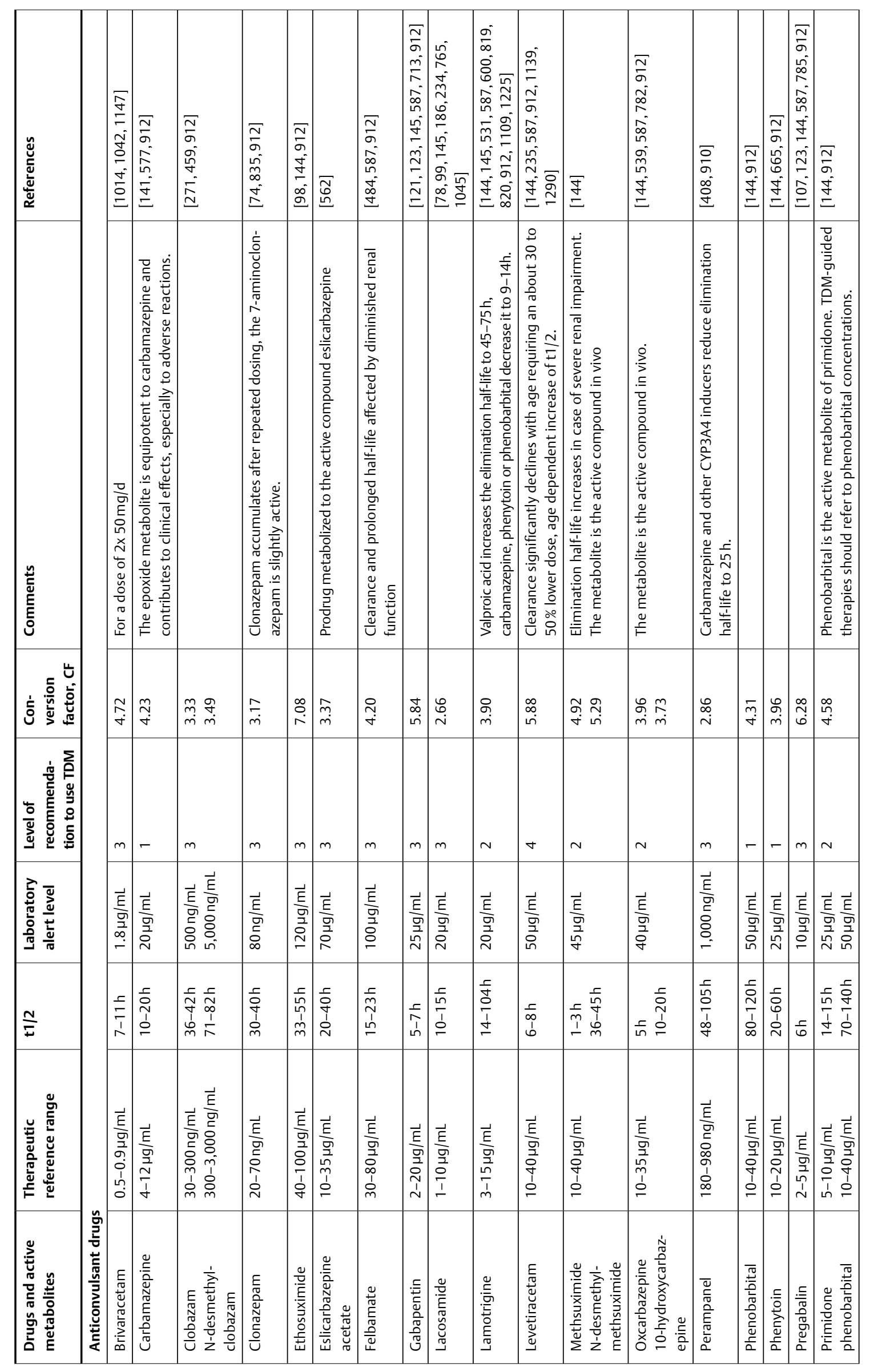




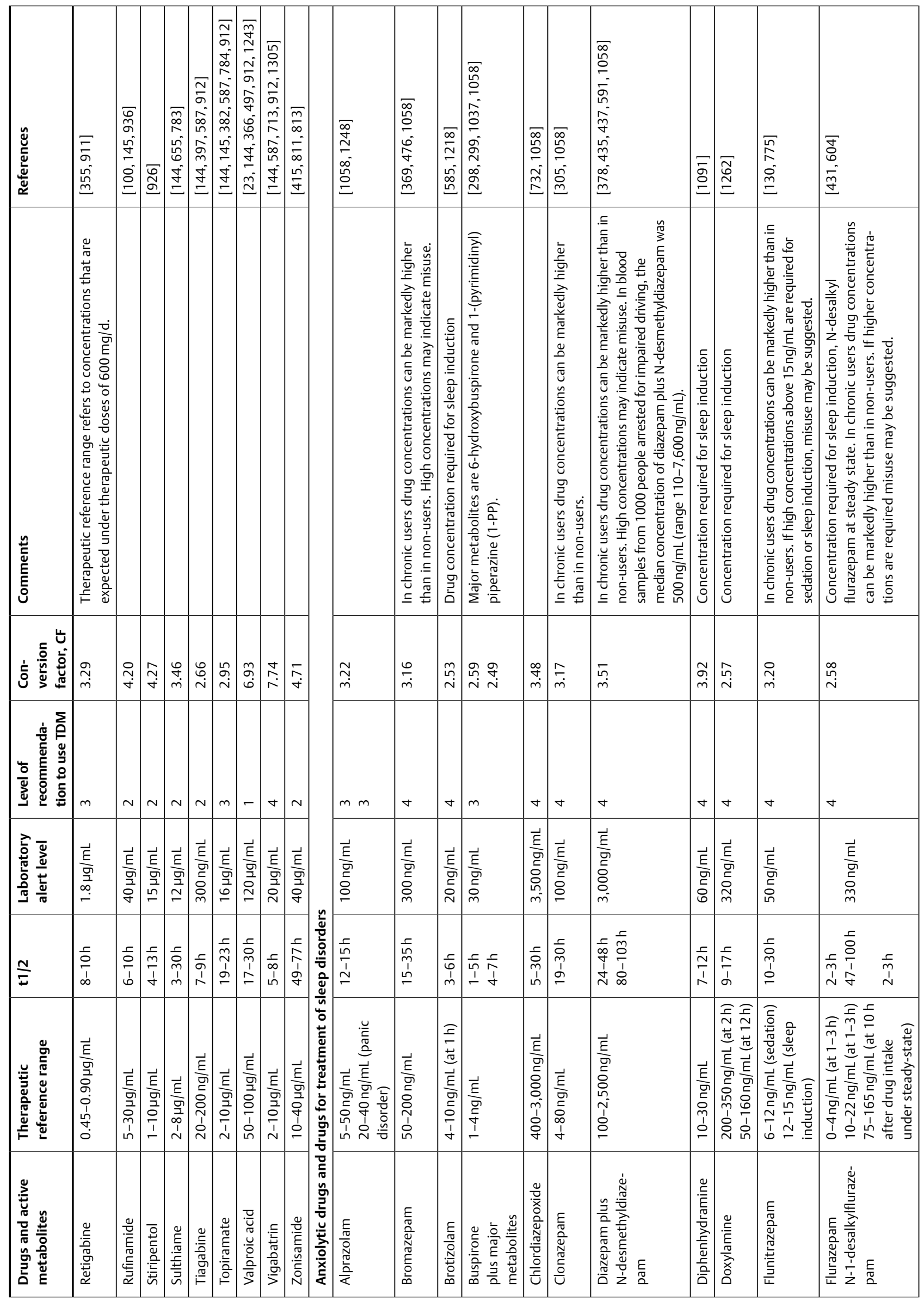




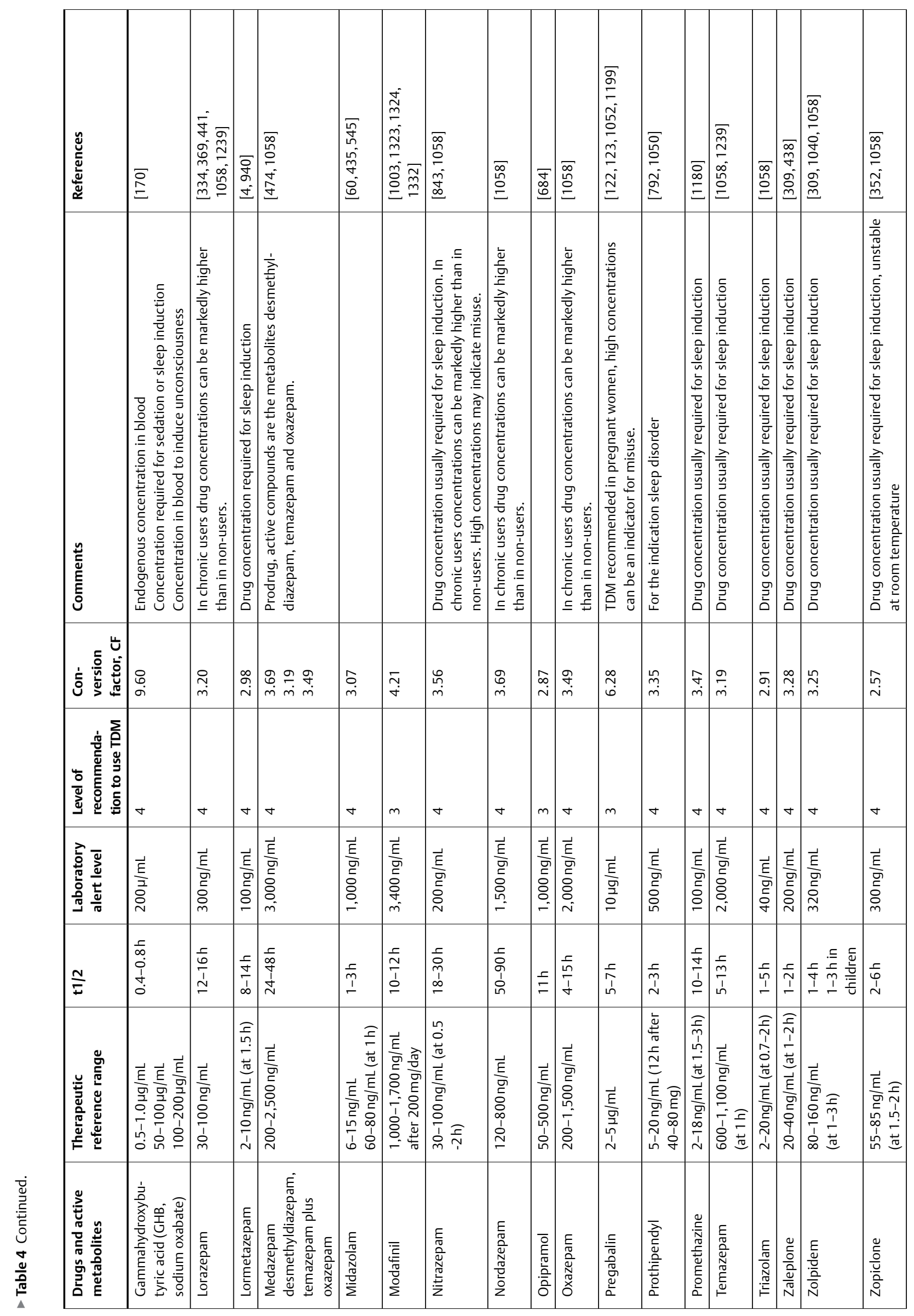




\begin{tabular}{|c|c|c|c|c|c|c|c|c|c|c|c|c|}
\hline 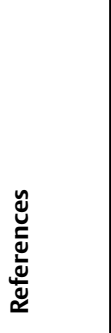 & & 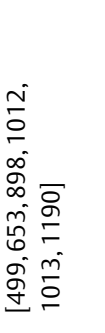 & 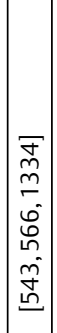 & 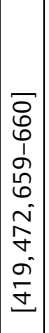 & 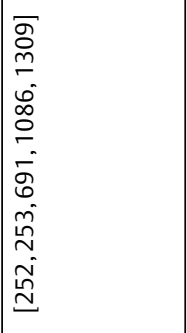 & & 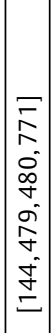 & 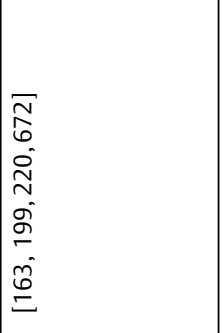 & $\begin{array}{l}\bar{\infty} \\
\infty \\
0 \\
\tilde{\infty} \\
\underline{=}\end{array}$ & 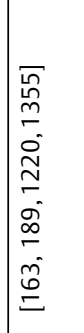 & $\begin{array}{l}\bar{F} \\
\bar{m} \\
\dot{0} \\
\underline{m}\end{array}$ & 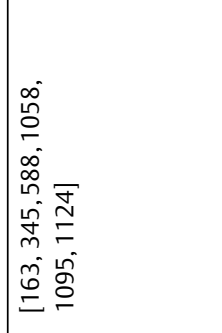 \\
\hline 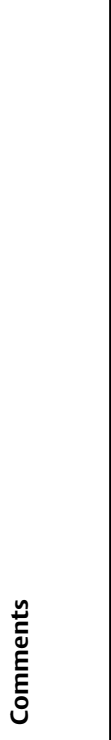 & & 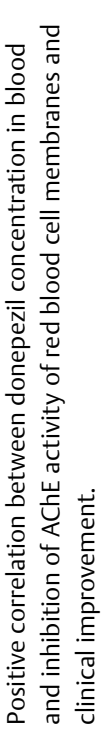 & 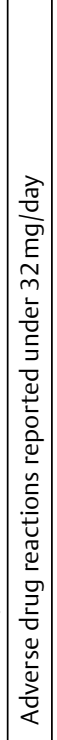 & 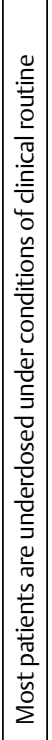 & 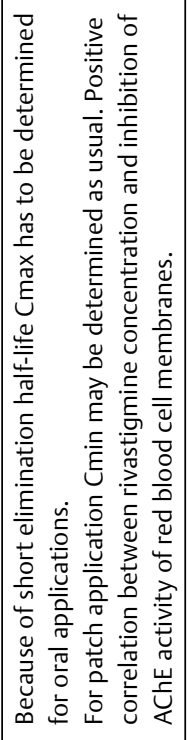 & & & 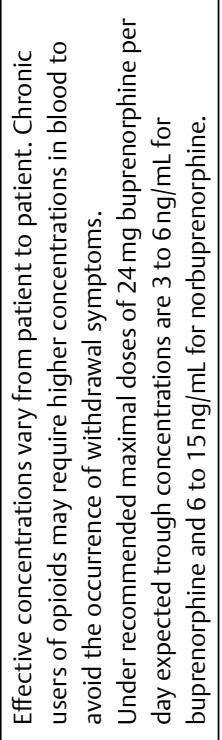 & 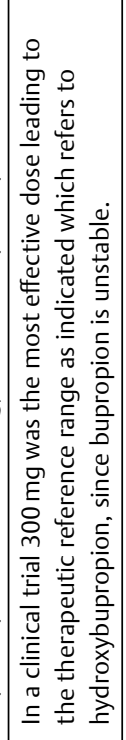 & 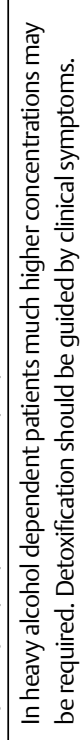 & 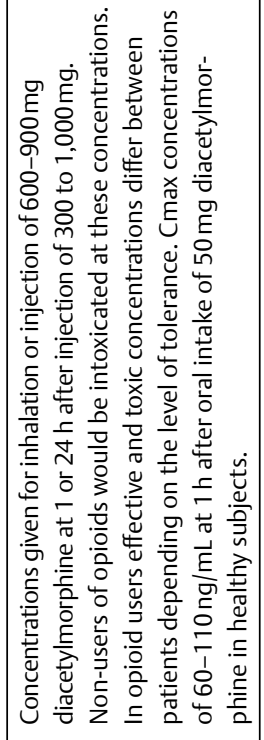 & 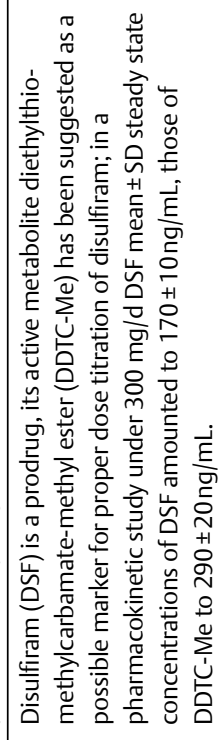 \\
\hline ن & & & $\mid \begin{array}{l}\infty \\
\stackrel{\infty}{m} \\
\dot{m}\end{array}$ & 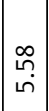 & $\underset{+}{\stackrel{8}{+}}$ & & $\begin{array}{l}\infty \\
0 \\
\infty \\
\infty\end{array}$ & $\stackrel{\substack{n \\
\sim}}{n}$ & 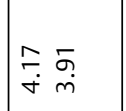 & $\frac{9}{6}$ & 슬 & $\begin{array}{l}\hat{m} \\
m \\
m\end{array}$ \\
\hline 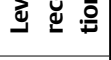 & & $\sim$ & $m$ & $m$ & $m$ & & $m$ & $\sim$ & $\sim$ & t & t & $m$ \\
\hline 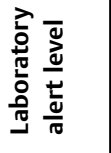 & & 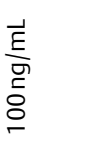 & 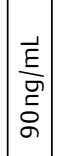 & 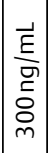 & \begin{tabular}{|l}
$\vec{E}$ \\
$\frac{\vec{E}}{9}$ \\
$\bar{\sigma}$ \\
$\dot{\sigma}$
\end{tabular} & & 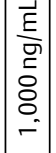 & $\begin{array}{l}\vec{E} \\
\frac{\vec{E}}{9} \\
\underline{\underline{O}} \\
\underline{\square}\end{array}$ & 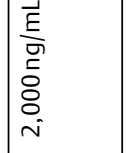 & & & 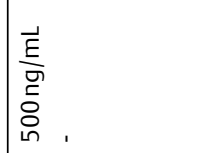 \\
\hline$\frac{N}{I}$ & & $\begin{array}{l}5 \\
\text { o } \\
0 \\
1 \\
\end{array}$ & \begin{tabular}{l|}
5 \\
\\
\\
1 \\
$\infty$
\end{tabular} & $\mid \begin{array}{l}5 \\
8 \\
0 \\
1 \\
0 \\
0\end{array}$ & $\frac{I}{I}$ & & $\begin{array}{c}\frac{\tilde{m}}{m} \\
\hat{m} \\
m\end{array}$ & 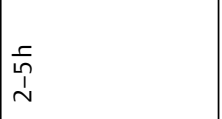 & 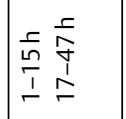 & $\begin{array}{l}\frac{1}{4} \\
\dot{1} \\
\sim\end{array}$ & 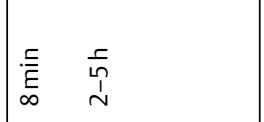 & $\begin{array}{l}\text { a } \\
\text { b } \\
b\end{array}$ \\
\hline 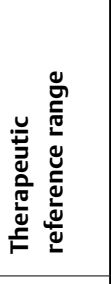 & & 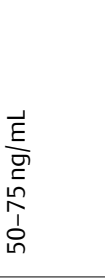 & 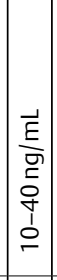 & 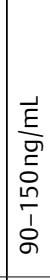 & 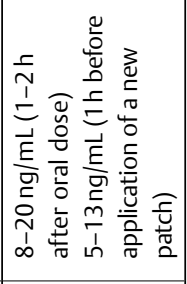 & 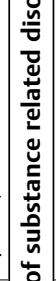 & 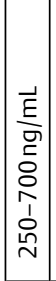 & $\begin{array}{l}\overrightarrow{\underline{E}} \\
\frac{\vec{g}}{\vec{g}} \\
\vec{m} \\
\stackrel{1}{-}\end{array}$ & 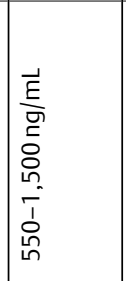 & 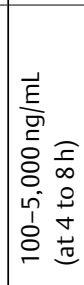 & 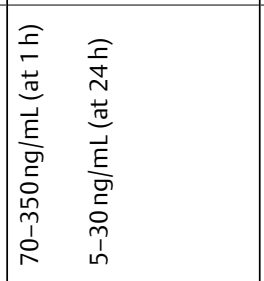 & 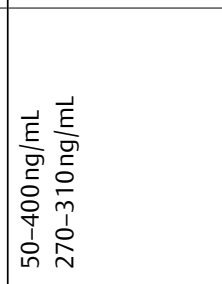 \\
\hline 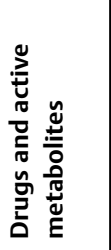 & 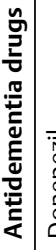 & 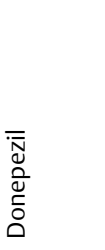 & 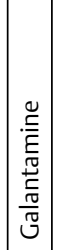 & 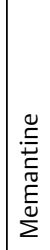 & 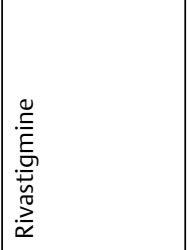 & 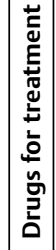 & 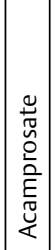 & 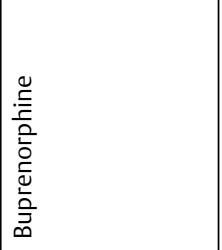 & 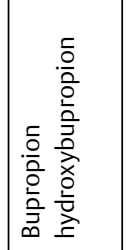 & 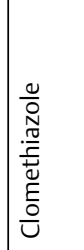 & 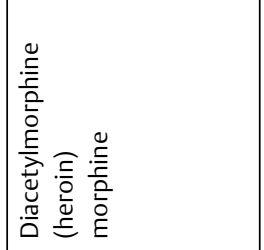 & 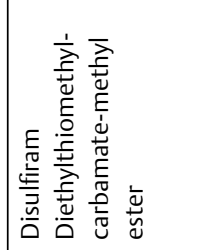 \\
\hline
\end{tabular}




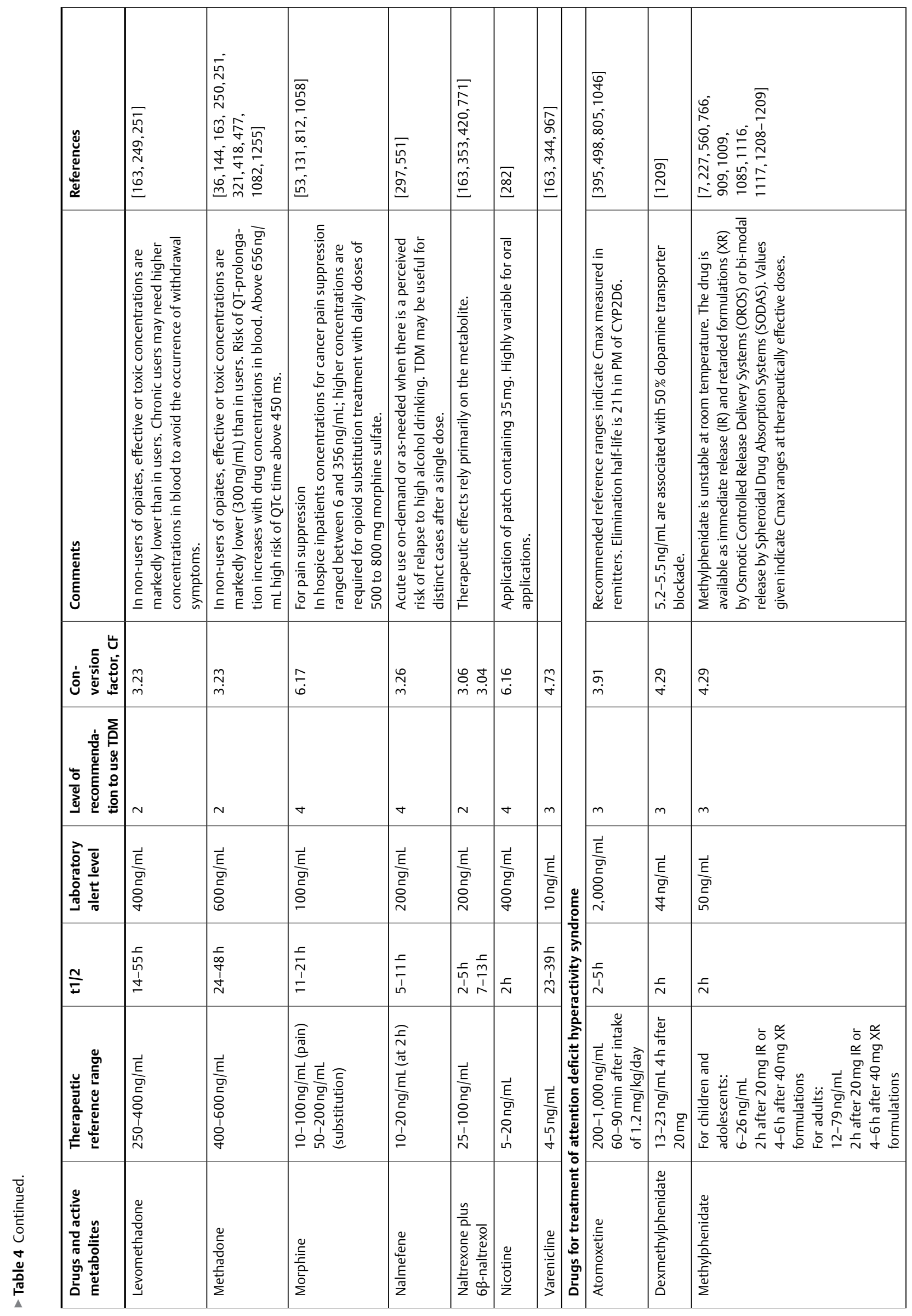

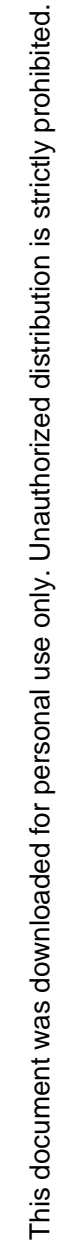




\begin{tabular}{|c|c|c|c|c|c|c|c|c|c|c|c|c|c|c|}
\hline 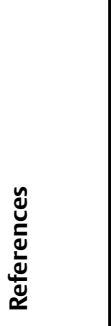 & & 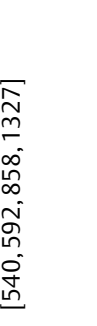 & 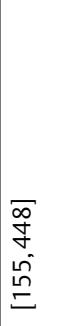 & $\begin{array}{l}\bar{D} \\
\stackrel{\infty}{=}\end{array}$ & $\begin{array}{l}\bar{F} \\
\hat{\sigma} \\
\sigma \\
\bar{D} \\
\underline{D}\end{array}$ & $\begin{array}{l}\bar{\Omega} \\
\stackrel{\Sigma}{\underline{a}}\end{array}$ & $\begin{array}{l}\overline{\mathscr{N}} \\
\stackrel{0}{1} \\
\tilde{\sim} \\
\tilde{\Xi}\end{array}$ & 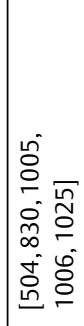 & 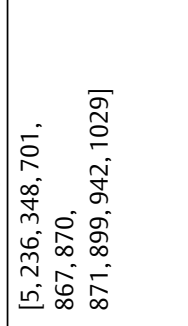 & $\begin{array}{l}\bar{d} \\
\tilde{n} \\
\bar{a} \\
\underline{a}\end{array}$ & 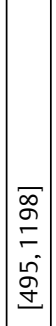 & $\underset{\substack{n \\
m}}{\stackrel{n}{n}}$ & 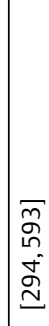 & 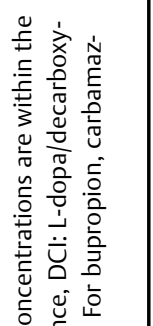 \\
\hline 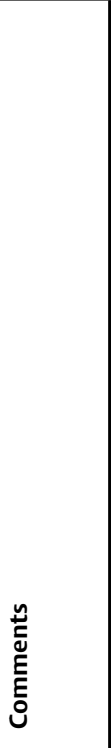 & & 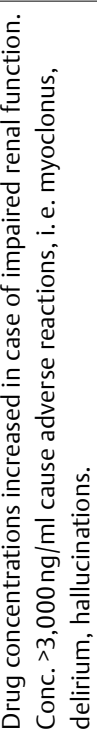 & & & 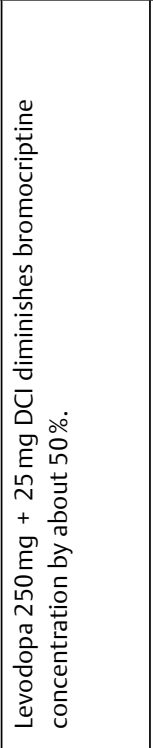 & 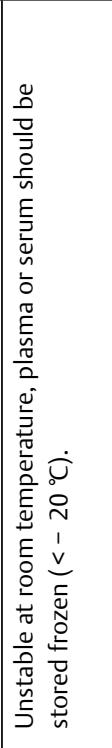 & 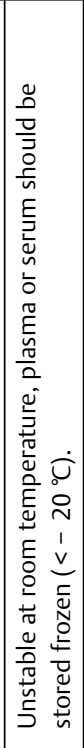 & 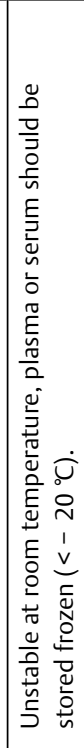 & 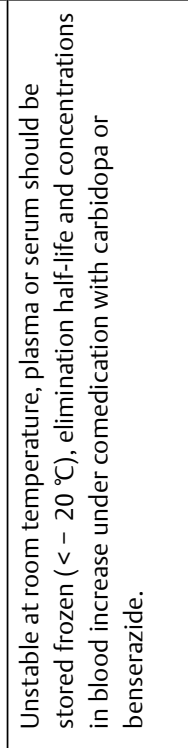 & 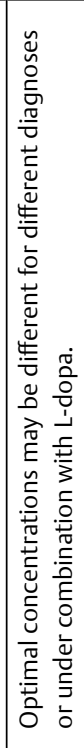 & & 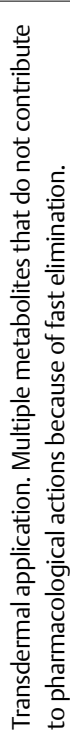 & & 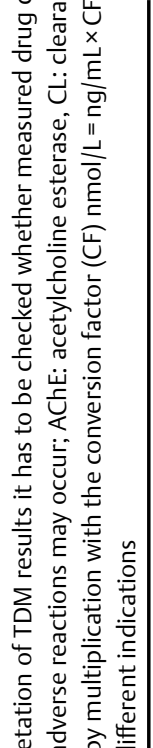 \\
\hline 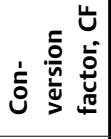 & & 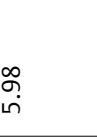 & 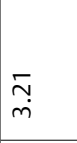 & $\begin{array}{l}\dot{O} \\
\dot{m}\end{array}$ & $\stackrel{\stackrel{n}{\leftrightarrow}}{-}$ & $\stackrel{\bar{N}}{\stackrel{N}{N}}$ & $\underset{f}{\stackrel{f}{*}}$ & $\stackrel{\infty}{\stackrel{\infty}{m}}$ & 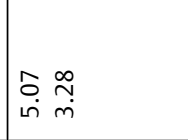 & 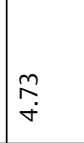 & $\mid \begin{array}{l}\dot{1} \\
\infty \\
\dot{m}\end{array}$ & $\stackrel{\bar{m}}{m}$ & $\begin{array}{l}\stackrel{\ell}{0} \\
\dot{m}\end{array}$ & 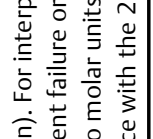 \\
\hline 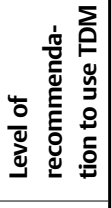 & & m & $m$ & $m$ & $m$ & $m$ & $m$ & $m$ & $m$ & $m$ & $m$ & $m$ & $m$ & 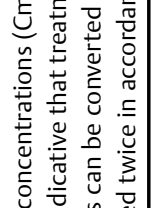 \\
\hline 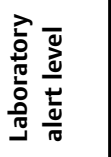 & & 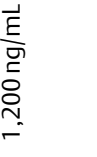 & $\begin{array}{l}\overrightarrow{\vec{E}} \\
\underline{\underline{G}} \\
\underline{m} \\
\underline{m}\end{array}$ & \begin{tabular}{|l}
$\vec{E}$ \\
$\frac{\vec{E}}{E}$ \\
$\vec{g}$
\end{tabular} & $\begin{array}{l}\vec{E} \\
\frac{\vec{E}}{9} \\
\stackrel{D}{\infty}\end{array}$ & \begin{tabular}{|l}
$\vec{E}$ \\
जू \\
Dे \\
D \\
m
\end{tabular} & \begin{tabular}{|l}
$\vec{E}$ \\
$\frac{\vec{E}}{⿹}$ \\
$\bar{\Xi}$ \\
$\dot{y}$
\end{tabular} & $\frac{\vec{E}}{\frac{\vec{V}}{N}}$ & 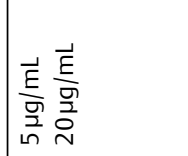 & $\begin{array}{l}\vec{E} \\
\stackrel{\vec{E}}{G} \\
\stackrel{n}{n} \\
\underline{r}\end{array}$ & 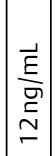 & $\begin{array}{l}\frac{\vec{E}}{\sigma} \\
\text { ज }\end{array}$ & $\begin{array}{l}\overrightarrow{\underline{E}} \\
\frac{\mathrm{g}}{2} \\
\stackrel{\underline{V}}{L}\end{array}$ & 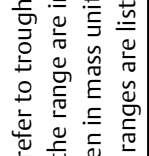 \\
\hline$\frac{N}{I}$ & & $\begin{array}{l}\frac{5}{5} \\
\frac{1}{1} \\
\\
\end{array}$ & 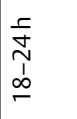 & 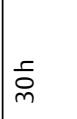 & 京 & $\begin{array}{l}5 \\
\infty \\
0 \\
1 \\
1 \\
6 \\
6\end{array}$ & $\stackrel{\check{N}}{\mathrm{~N}}$ & 点 & $\stackrel{\frac{n}{m}}{\underline{1}} \stackrel{\frac{n}{N}}{2}$ & $\begin{array}{l}\stackrel{\tilde{N}}{\tilde{1}} \\
\infty\end{array}$ & $\left|\begin{array}{c}\frac{c}{0} \\
\frac{1}{m}\end{array}\right|$ & $\begin{array}{l}\frac{5}{\hat{1}} \\
\underline{1}\end{array}$ & $\frac{\check{n}}{\sim}$ & 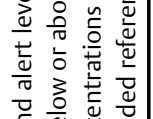 \\
\hline 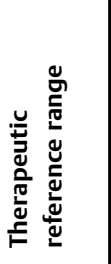 & & 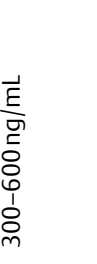 & 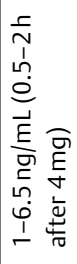 & 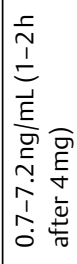 & 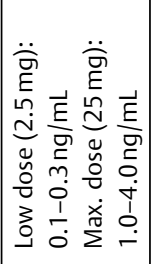 & 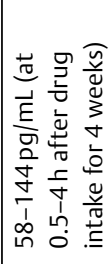 & 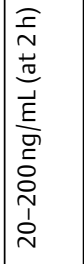 & 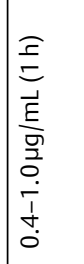 & 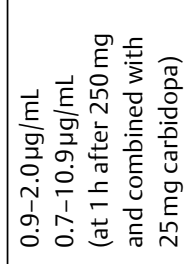 & 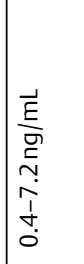 & $\begin{array}{c}\vec{E} \\
\vec{E} \\
\vec{E} \\
0 \\
\dot{b} \\
\dot{1} \\
\dot{0}\end{array}$ & \begin{tabular}{|l}
$\vec{E}$ \\
$\underline{E}$ \\
$\underline{\Xi}$ \\
$\hat{0}$ \\
$\dot{1}$ \\
$\frac{1}{0}$
\end{tabular} & 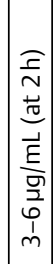 & 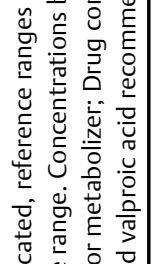 \\
\hline 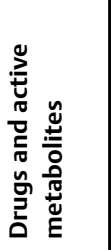 & 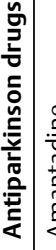 & 部 & 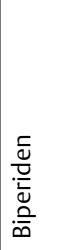 & 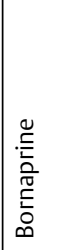 & 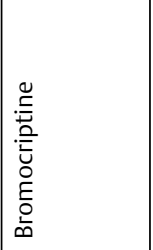 & 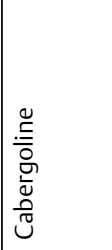 & $\begin{array}{l}\frac{\pi}{2} \\
\frac{0}{0} \frac{0}{2} \\
\frac{2}{2}\end{array}$ & 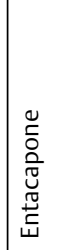 & 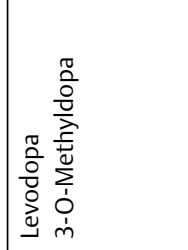 & 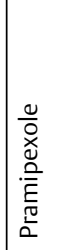 & 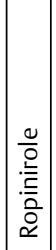 & 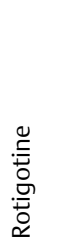 & 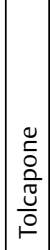 & 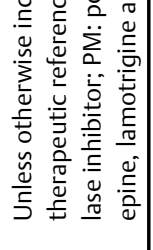 \\
\hline
\end{tabular}


for the treatment of anxiety or obsessive compulsive disorder or chronic pain, and antipsychotic drugs are approved for the treatment of affective disorders. Little information is available on optimal drug concentrations in blood for these indications. Exceptions are carbamazepine, lamotrigine and valproic acid (valproate), which are therefore sometimes listed twice in $>$ Table 4. It should be mentioned that studies are on the way to evaluate therapeutic reference ranges for children or adolescent patients $[328,399,654,1177,1314]$. For elderly patients, there is an urgent need to conduct similar studies.

When new drugs become available, it is a major handicap for TDM guided pharmacotherapy that therapeutic reference ranges are unclear. Estimation of therapeutic reference ranges is not required for drug approval, and therefore they are rarely established. To be able to make a meaningful use of TDM in spite of this missing link, we propose for these situations to establish a provisional reference range.

\section{Recommendation}

As long as valid data on therapeutic reference ranges do not exist, we recommend determination of the arithmetric mean \pm standard deviation of drug concentrations in blood of responders to the neuropsychiatric medication. The mean \pm SD range should be used as preliminary therapeutic reference range. Further (prospective or observational) studies must verify or correct this range.

\subsubsection{Estimation of the lower limit of the therapeutic reference range}

Whenever possible, the lower limit of a drug's therapeutic range should be based on studies estimating the relationship between a drug's concentration in blood and clinical effectiveness. Below the lower limit, drug effects are not significantly different from placebo. The optimal study design to evaluate the lower limit is a prospective double-blind randomized controlled trial where patients are treated with drug doses that result in a predefined blood concentration range of the drug. An almost optimal study design was applied by Van der Zwaag and co-workers on patients treated with clozapine [1241]. Clozapine concentrations in blood were titrated to $50-150 \mathrm{ng} / \mathrm{mL}, 200-300 \mathrm{ng} / \mathrm{mL}$ or $350-450 \mathrm{ng} / \mathrm{mL}$. Significant therapeutic superiority was found for middle and high concentrations compared to low concentrations of clozapine. A similar design was applied to a blood level study comparing imipramine and mirtazapine [162]. To conduct such studies, however, is a considerable logistic challenge. Fixed dose studies are feasible and therefore preferable for the evaluation of the lower limit [1222, 1224].

To estimate the threshold value of a therapeutic reference range, receiver operating characteristic (ROC) analysis has proven helpful [483]. A ROC plot allows the identification of a cut-off value that separates responders from non-responders and estimates the sensitivity and specificity of the parameter "drug concentration in blood". The usefulness of ROC analysis has been demonstrated for a number of antipsychotic and antidepressant drugs [829, 928, 934, 1274].
2.1.2 Estimation of the upper limit of the therapeutic reference range

In the first study on TDM in psychiatry [52], an inverse U-shaped relationship between blood concentrations and clinical effects was reported for nortriptyline. The lack of therapeutic improvement at high concentrations was attributed to the mechanism of action of the tricyclic antidepressant drug on monoaminergic neurons. According to current knowledge, however, it seems more likely that reduced amelioration at high concentrations is due to nortriptyline's adverse reactions. The upper limit of the therapeutic range is therefore often defined by the increased risk of adverse drug reactions, also in these guidelines. Correlations to drug concentrations in blood were shown for motor symptoms of antipsychotic drugs [973] and for unwanted effects of tricyclic antidepressant drugs $[261,465]$. For paroxetine, a positive correlation was found between the drug concentration in blood and serotonin syndrome symptoms [503]. For citalopram, it was shown that adverse drug reactions correlated inversely with clearance of the drug [1339]. When such data are available, it is possible to apply ROC analysis for the calculation of the upper limit of the therapeutic range [829]. For many neuropsychiatric drugs listed in $>$ Table 4, however, valid data on both the concentration in blood and the incidence of adverse drug reactions, are lacking. Case reports on tolerability problems or intoxications mostly do not include drug concentration measurements. Sporadic reports on fatal cases and intoxications are of limited value. When reported blood concentrations have caused death, the drug level is mostly far above the concentration that is associated with maximal therapeutic effects $[983,1132]$. Moreover, post mortem redistribution of drugs from or into the blood can lead to dramatic changes in blood levels [671, 948], and the direction of the change does not follow a general rule [616]. Because of these limitations estimation of an upper threshold level above which tolerability decreases or the risk of intoxication increases is more difficult than estimation of the lower threshold level, especially for drugs with a broad therapeutic index like SSRIs. Therefore, many upper threshold values listed in $>$ Table 4 refer to concentrations where maximum efficiency is expected. In these guidelines, upper limit threshold levels were mostly obtained by calculation of expected dose-related drug concentrations in blood (Cmin) attained under approved maximal doses.

\subsubsection{From population-based to subject-based reference values}

All therapeutic reference ranges listed in $>$ Table $\mathbf{4}$ are populationbased. The population-derived ranges constitute descriptive statistical values not necessarily applicable to all patients. Optimal neuropsychopharmacotherapy should try to identify a patient's "individual optimal therapeutic concentration range" to guide the treatment $[96,955]$. Furthermore, the stage of the mental disorder also determines the optimal drug concentration. For lithium, it has been shown that the optimal concentration range depends on whether the patient is in an acute manic episode or under maintenance therapy [1076]. For clozapine, Gaertner and colleagues [391] determined individual optimal drug concentrations in blood required for stable remission for every patient under maintenance therapy in a relapse prevention study and found that the antipsychotic drug concentration in maintenance therapy can be up to $40 \%$ lower than that needed for the treatment of an acute schizophrenic episode. 


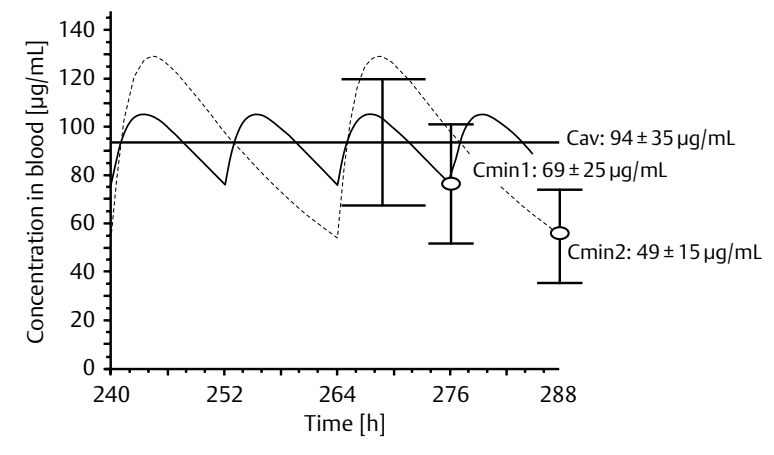

- Fig. 3 Time to concentration curves expected under steady state conditions for valproic acid in blood after daily oral doses of $900 \mathrm{mg}$ with dosing intervals of either $12 \mathrm{~h}$ (solid, Cmin 1 ) or $24 \mathrm{~h}$ (hatched, Cmin2). The average elimination half-life (t1/2) of valproic acid is $14 \mathrm{~h}$. Circles and error bars indicate expected trough ( $\mathrm{Cmin})$ and average concentrations (Cav) \pm standard deviation (SD).

\section{Recommendation}

It can be useful to measure the drug concentration in blood when the patient has attained the desired clinical outcome. This drug concentration can be regarded as optimal concentration for the individual patient. In case of symptom aggravation, relapse or adverse drug reactions, the value is helpful to find out whether non-adherence or pharmacokinetic alterations have occurred that can explain clinical impairment.

\subsubsection{Laboratory alert level}

For most neuropsychiatric drugs shown in $>$ Table 4, concentrations in blood with an increased risk of toxicity are normally much higher than the upper threshold levels of the therapeutic reference ranges. In the present guidelines, a "laboratory alert level" is defined as follows:

\section{Definition}

The "laboratory alert levels" reported in this guideline ( Table 4) indicate drug concentrations above the recommended therapeutic reference range that oblige the laboratory to feedback immediately to the prescribing physician. For some drugs, the alert levels are based on reports on severe adverse drug reactions or intoxications that were supplemented by concentration measurements. Mostly, however, the alert level was arbitrarily defined as a drug concentration in blood that is 2-fold higher than the upper limit of the therapeutic reference range. The laboratory alert should lead to dose reduction when the patient exhibits signs of adverse drug reactions. When the high drug concentration is well tolerated by the patient and if a dose reduction bears the risk of symptom exacerbation, the dose should remain unchanged. The clinical decision, especially in case of unchanged dosage in the face of an alert level that was reached or exceeded, needs to be documented in the medical file.

\subsection{The dose-related reference range}

For the interpretation of TDM results, there is a second concentration range besides the therapeutic reference range, the so called dose-related reference range. The use of the therapeutic reference range is a pharmacodynamic approach. Application of the doserelated reference range is a pharmacokinetic approach. It compares a measured drug concentration with a theoretically expected drug concentration range. Referring to pharmacokinetic studies, preferentially on a population of patients without co-medication or pharmacogenetic abnormalities ("normal” patients), the average steady-state concentration (Cav) of a drug expected in a normal patient can be calculated when the daily maintenance dose (Dm), the dosing interval (di), the total clearance $(\mathrm{CL})$ and the bioavailability (F) are known:

$$
\mathrm{Cav}=(\mathrm{Dm} / \mathrm{di}) \times(\mathrm{F} / \mathrm{CL})
$$

Dose and dosing interval are known from the prescription, pharmacokinetic parameters are available from pharmacokinetic trials. Using the daily dose $(1 \mathrm{mg} / 24 \mathrm{~h}=1,000,000 \mathrm{ng} / 1440 \mathrm{~min})$, the standard deviation (SD) of the total apparent clearance CL/F (mL) $\mathrm{min}$ ), that is also reported in the literature, it is possible to calculate Cav \pm SD ( $\mathrm{ng} / \mathrm{mL}$ ) by Eq. (1). For the calculation, the dimensions of the different parameters must be considered and all doses have to be converted to $\mathrm{ng}$, all volumes to $\mathrm{mL}$ and time intervals to min. When a $C L / F$ value of $100 \pm 50 \mathrm{~mL} /$ min was reported, the coefficient of variation is $50 \%$, then Cav amounts to $139 \mathrm{ng} / \mathrm{mL}$ for a dose of $20 \mathrm{mg} /$ day (i. e., $(20,000,000 \mathrm{ng} / 1440 \mathrm{~min}){ }^{*}(1 /(100 \mathrm{~mL} /$ $\min ))=139 \mathrm{ng} / \mathrm{mL}$ ), SD of Cav will be $69 \mathrm{ng} / \mathrm{mL}$ and the Cav \pm SD ranges from 70 to $208 \mathrm{ng} / \mathrm{mL}$. Assuming a dosing interval of $24 \mathrm{~h}$, i. e., once daily (quaque die, q.d.) dosing , the Cav \pm SD range was proposed as dose-related reference range by Haen and colleagues $[470,471]$. The mean - SD was considered as lower and the mean + SD as upper limit of this range. Statistically, this range contains $68 \%$ of concentrations determined under normal conditions in the blood of a population that consists of 18-65 years old individuals. For the 2011 guidelines [524], apparent total clearance (CL/F) data \pm SD were extracted from the literature for 83 neuropsychiatric drugs for calculation of dose factors. Multiplying these factors \pm SD by the daily dose, dose-related reference ranges were calculated and used for the interpretation of TDM results. When a patient's drug concentration measured by TDM was found within the dose-related reference range, the concentration was defined as normal. Concentrations above or below the range were considered as signals indicating potential abnormalities such as partial non-adherence, drug-drug interactions, genetic polymorphisms of drug metabolizing enzymes or diseases of organs involved in drug elimination.

The concept of the dose-related reference range worked. Many incompletely adherent patients or patients with pharmacokinetic abnormalities could be identified [470]. The average steady-state concentration equation is valid and useful when the drug's elimination half-life ( $\mathrm{t} 1 / 2)$ is long compared to the dosing interval. Howev$\mathrm{er}$, when $\mathrm{t} 1 / 2$ is short and the dosing interval is longer than $\mathrm{t} 1 / 2$, values calculated by Eq. (1) are poorly predictive for the $\mathrm{Cmin}$ values used for TDM. This problem is illustrated in $\mathbf{F i g . ~} \mathbf{3}$ for valproic acid which has a $\mathrm{t} 1 / 2$ of $14 \mathrm{~h}$ and is applied either once or twice daily. 
Under daily doses of $900 \mathrm{mg}$, the dose-related reference range of valproic acid computed by Eq. (1) amounts to $94 \pm 35 \mu \mathrm{g} / \mathrm{mL}$, independent of the dosing interval. Time to concentration curves, however, show that the trough concentrations are lower than Cav, $49 \pm 15 \mu \mathrm{g} / \mathrm{mL}$ if the dosing schedule is a single $900 \mathrm{mg}$ dose per day. It amounts to $69 \pm 25 \mu \mathrm{g} / \mathrm{mL}$ if the daily dose of $900 \mathrm{mg} / \mathrm{d}$ is administered in two doses of $450 \mathrm{mg}$ each. Cav \pm SD ranges match with $\mathrm{Cmin} \pm$ SD ranges for dosing intervals $<14 \mathrm{~h}$. Therefore, computed Cav can be considered as an appropriate predictor for an expected drug concentration in blood. Under a single dose per day schedule, however, $\mathrm{Cmin}$ at $24 \mathrm{~h}$ after the last dose is by $54 \%$ lower than Cav. As explained here for valproic acid as an example, this limitation must be considered when using Eq. (1) based calculations of dose-related reference ranges. Depending on the dosing interval, this limitation can be relevant for multiple drugs, e. g., duloxetine, paroxetine, venlafaxine, amisulpride, paliperidone, quetiapine, lithium, valproic acid, zopiclone, atomoxetine or naltrexone. When dosing intervals are longer than $t 1 / 2$, computed values are by more than $30 \%$ lower for $\mathrm{Cmin}$ than for Cav. Overall, this applies for $32 \%$ of the compounds listed in $>$ Table 5.

Moreover, there is another limitation of Cav based calculations. The validity of the dose-related reference range cannot be easily verified by measurements which, in contrast, is possible for $\mathrm{Cmin}$, because TDM is based on the measurement of a drug's minimal ("trough") blood concentration. Cav is by definition the area under the time to concentration curve (AUC) divided by the dosing interval. It cannot be attributed to a distinct time point like $\mathrm{Cmin}$ which is necessary for the timing of venipuncture. Another limitation of Cav based calculations is neglection of fluctuations of drug levels over the day as shown in $\mathbf{F i g} \mathbf{2}$ which can be important for a drug's tolerability and efficacy [206].

Because of these limitations, it was decided to modify the calculation of dose-related reference ranges for this update. Without going into the details described in textbooks on pharmacokinetics (see e.g., [77, 306]), steady-state concentrations can be calculated by extension of Eq. (1) and applying the Bateman function. Gex-Fabry and colleagues [404] used this approach and described a function for the postabsorptive phase, which is the interval between tmax, the time of maximal drug concentration, and tmin, the time of $\mathrm{Cmin}$, to calculate concentration during the elimination phase.

Assuming a one-compartment model and an exponential decrease of drug concentration in blood, an expected steady-state drug concentration Ct can be computed for any time point during the postabsorptive phase as follows:

$$
C t=[(D m / d i) \times(F / C L)] \times\left[(k e \times d i) /\left(1-e^{-k e \times d i}\right)\right] \times\left(e^{-k e \times t}\right)
$$

where $\mathrm{Dm}$ is the dose under steady-state conditions, termed maintenance dose, $\mathrm{CL} / \mathrm{F}$ apparent total clearance (for calculation used as reciprocal value), di dosing interval, ke elimination rate constant, to be calculated from the elimination half-life, $t_{1 / 2}$, by $k e=\ln 2 / t_{1 / 2}$, and $t$ the time of blood withdrawal.

Assuming di as $24 \mathrm{~h}$ and $\mathrm{t}$ as time interval between intake of the last dose and blood withdrawal as $\Delta t$, Eq. (3) can be used to estimate an expected $\mathrm{Cmin}$ as follows:
$C \min =(\operatorname{Dm} / 24) \times(F / C L) \times\left[(k e \times 24) /\left(1-e^{-k e \times 24}\right)\right] \times\left(e^{-k e \times \Delta t}\right)$

Drug concentrations expected by TDM measurements can thus be computed by daily dose, $\mathrm{CL} / \mathrm{F}, \mathrm{t}_{1 / 2}$ and time interval between last dose and blood withdrawal $\Delta t$. As for the calculation of Cav, the pharmacokinetic parameters $\mathrm{CL} / \mathrm{F}$ and $\mathrm{t} 1 / 2$ are available from pharmacokinetic trials, daily dose and $\Delta \mathrm{t}$ are fixed by the prescriber.

Using part of Eq. (3), a DRC factor can be defined and computed, e.g., by MS-Excel software, for drugs with known CL/F and t1/2 as follows.

DRC factor $=(F / C L) \times\left[(k e \times 24) /\left(1-\mathrm{e}^{-\mathrm{ke} \times 24}\right)\right] \times\left(\mathrm{e}^{-\mathrm{ke} \times \Delta \mathrm{t}}\right)$

Expected $\mathrm{Cmin}$ of a given dose can then be calculated by multiplying the DRC factor by the daily dose. The limitations for prediction of theoretically expected $\mathrm{Cmin}$ in comparison to Cav are the more complex calculation procedure and the need to implement $\mathrm{t} 1 / 2$ which also varies between individuals. Since variability of $\mathrm{t} 1 / 2$ is probably caused by the same factors as variability of clearance, it was assumed for the TDM guidelines that the SD of mean drug concentrations measured in a population of adherent patients reflects normal variability of apparent total clearance (CL/F). Based on this assumption it was defined that the interindividual variability of a population's CL/F equals the variability of Cmin. The SD reported in the literature for $\mathrm{CL} / \mathrm{F}$ was thus propagated to $\mathrm{Cmin}$ to calculate expected mean \pm SD as dose-related reference range as done previously for Cav based calculations [471]. It was empirically tested whether this way of calculation predicts expected drug concentrations.

- Table 5 lists DRC factors for 172 compounds with inclusion of parent drugs, metabolites and active moiety. Factors were computed by Eq. (4) using pharmacokinetic data reported in the literature. Following recommended schedules of drug application, decisions were made to define $\Delta \mathrm{t}$. For a drug like citalopram or extended release $(X R)$ venlafaxine given once per day in the morning, $\Delta \mathrm{t}$ was $24 \mathrm{~h}$. For drugs like amitriptyline that is given normally in the morning and the evening, $\Delta \mathrm{t}$ was set at $12 \mathrm{~h}$. For hypnotic drugs given shortly before bedtime and blood withdrawal in the next morning, $\Delta \mathrm{t}$ was set at $10 \mathrm{~h}$. Listed factors can be used for calculation of the lower and the upper limit of the range by multiplying DRC factors low (= DRC factor $-\mathrm{SD}$ ) and high (= DRC factor + SD) by the daily dose to obtain the dose-related reference range. When drugs are given once or twice daily, DRC factors are given in $>$ Table $\mathbf{5}$ for $\Delta \mathrm{t}$ at 12 and $24 \mathrm{~h}$, respectively. For drugs like clomethiazole or modafinil where blood concentrations are not measured at tmin (no trough levels), DRC factors are given in > Table $\mathbf{5}$ at distinct time points when blood withdrawal is recommended.

The validity of these calculations, based on eqs. [(2]) to ([4]) and the pharmacokinetic parameters $\mathrm{CL} / \mathrm{F}$ and ke of pharmacokinetic studies on normal patients reported in the literature as well as recommended dosing interval and daily doses according to the SPC provided by the manufacturer, was controlled for plausibility using empirically obtained $\mathrm{Cmin}$ values reported for normal patients in TDM studies. Computed dose-related reference ranges were accepted when theoretical values were confirmed by empirical data. This was the case when the empirical mean $C$ min value was within the theoretical dose-related reference range. 


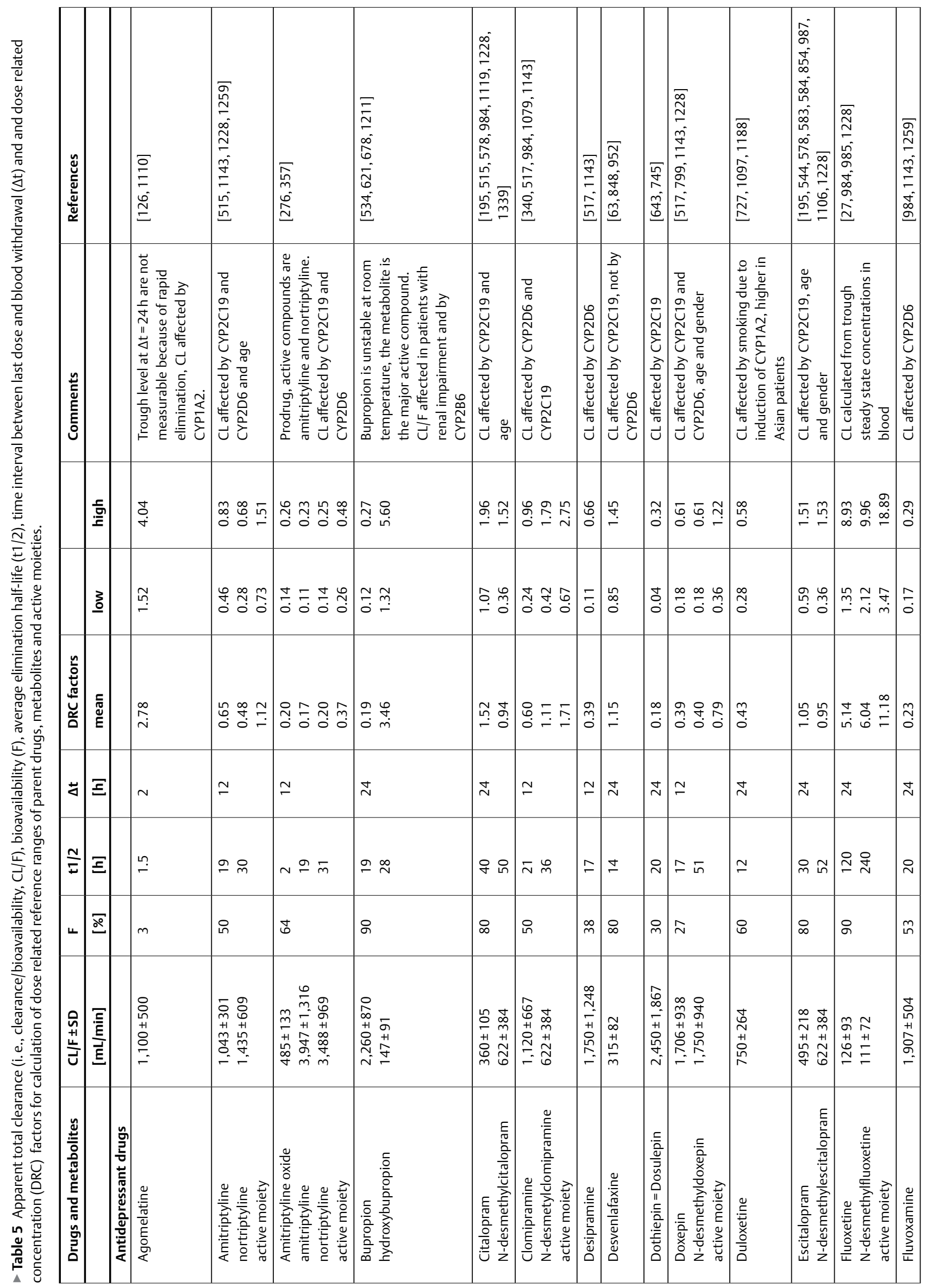




\begin{tabular}{|c|c|c|c|c|c|c|c|c|c|c|c|c|c|c|c|c|c|}
\hline 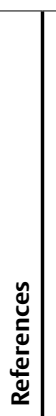 & & 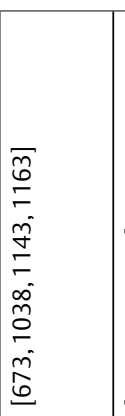 & 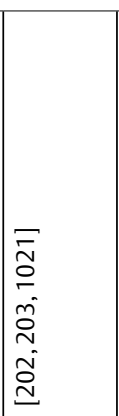 & 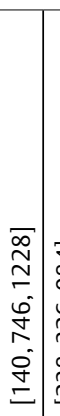 & 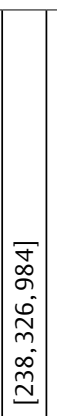 & $\underset{\widetilde{\Sigma}}{\bar{\Xi}}$ & 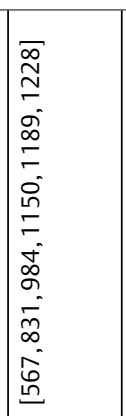 & $\mid$ & 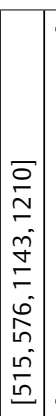 & 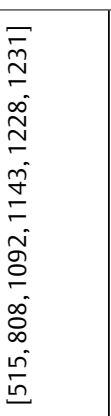 & 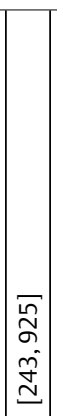 & 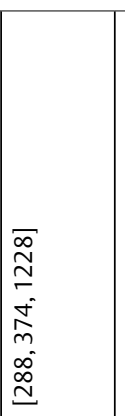 & 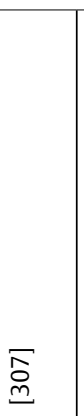 & $\stackrel{\bar{n}}{\stackrel{n}{\Sigma}}$ & 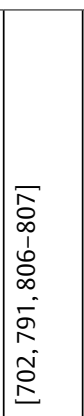 & $\begin{array}{l}\overline{\tilde{n}} \\
\tilde{\Sigma} \\
\tilde{N} \\
\tilde{\tilde{e}}\end{array}$ & 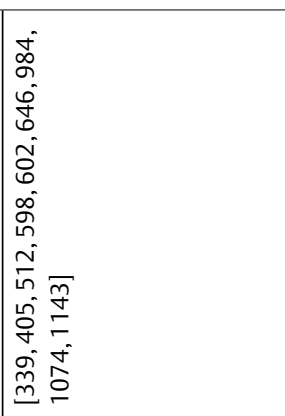 \\
\hline 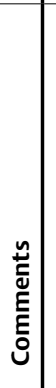 & & 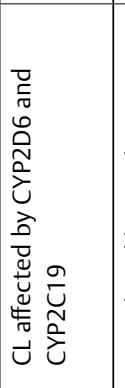 & 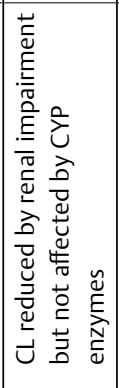 & 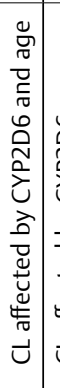 & 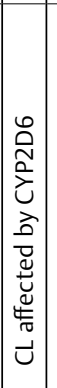 & 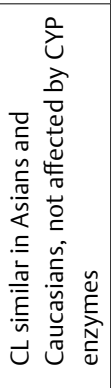 & 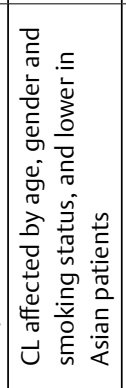 & 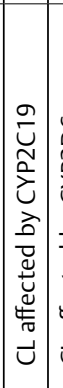 & 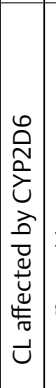 & 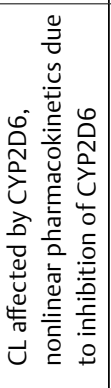 & & 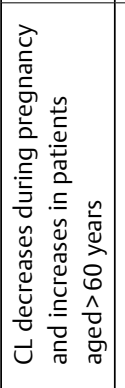 & 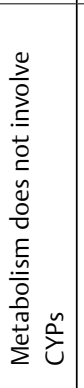 & 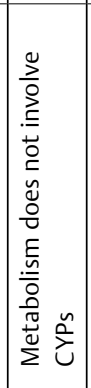 & 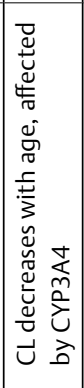 & 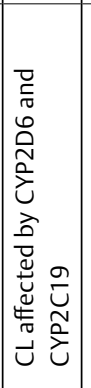 & 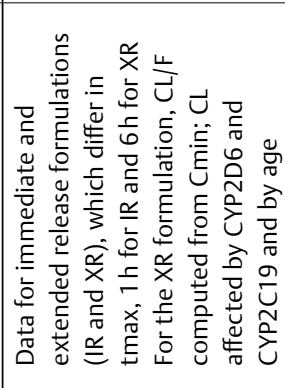 \\
\hline & 矛 & 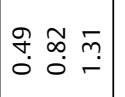 & 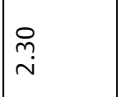 & $\stackrel{\substack{f \\
\hdashline \\
-}}{3}$ & $\stackrel{+}{\stackrel{+}{*}}$ & $\stackrel{\Delta}{\check{r}}$ & $\stackrel{m}{q}$ & $\mp$ & $\left|\begin{array}{l}\infty \\
\infty \\
0 \\
0\end{array}\right|$ & $\begin{array}{c}m \\
\infty \\
\infty\end{array}$ & 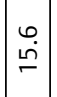 & 狊 & $\stackrel{\infty}{\stackrel{m}{\longrightarrow}}$ & $\tilde{m}$ & 空 & $\frac{\mathfrak{a}}{\hat{0}}$ & 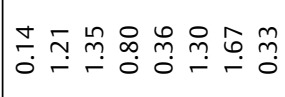 \\
\hline & $\underline{z}$ & 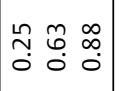 & 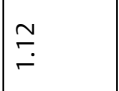 & ปี่ & $\stackrel{0}{0}$ & $\stackrel{m}{\infty}$ & $\stackrel{\infty}{\stackrel{\infty}{-}}$ & $\left|\begin{array}{l}\infty \\
0 \\
0\end{array}\right|$ & 色 & man & $\begin{array}{l}\text { के } \\
\text { in }\end{array}$ & 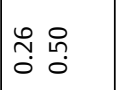 & $\stackrel{\substack{\infty \\
\infty}}{\infty}$ & $\frac{0}{0}$ & $\stackrel{\stackrel{n}{m}}{m}$ & f. & 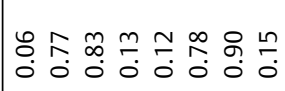 \\
\hline 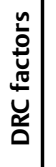 & 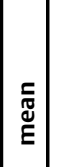 & \begin{tabular}{lll|}
$\hat{m}$ & $\stackrel{n}{2}$ & 0 \\
0 & 0 & 0 \\
0 & 0
\end{tabular} & $\stackrel{-}{i}$ & $\begin{array}{r}n \\
\mathscr{o} \\
0\end{array}$ & $\stackrel{m}{\dot{m}}$ & g. & $\underset{\substack{0 \\
i}}{i}$ & $\mid \begin{array}{c}0 \\
\infty \\
0 \\
0\end{array}$ & $\mid \begin{array}{l}1 \\
0 \\
0\end{array}$ & : & $\mid \begin{array}{l}\infty \\
\stackrel{0}{\ominus}\end{array}$ & $\underset{\substack{n \\
0}}{\stackrel{n}{0}}$ & $\stackrel{g}{\circ}$ & $\bar{i}$ & 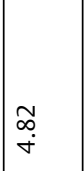 & $\bar{\sigma}$ & 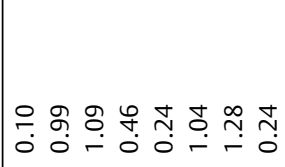 \\
\hline $\overrightarrow{4}$ & $\Xi$ & $\simeq$ & $\simeq$ & $\simeq$ & $\simeq$ & $\simeq$ & $\simeq$ & $\simeq$ & $\simeq$ & $\stackrel{d}{d}$ & $\simeq$ & $\stackrel{n}{d}$ & $\simeq$ & $\simeq$ & $\simeq$ & $\simeq$ & $\stackrel{ \pm}{\sim}$ \\
\hline$\frac{N}{E}$ & $\Xi$ & $\simeq \bar{N}$ & $\infty$ & q & $\approx$ & $\infty$ & in & 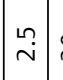 & $\stackrel{m}{.}$ & $\stackrel{9}{-}$ & $\circ$ & $\stackrel{N}{\sim}$ & $m$ & m & $\wedge$ & $\stackrel{\Xi}{\sim}$ & $0=\wedge=\stackrel{N}{n}$ \\
\hline\llcorner & ๖ं & mे & \& & 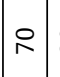 & $\dot{m}$ & $\infty$ & in & P & in & ర్ & 8 & 8 & $\stackrel{\circ}{\circ}$ & $\stackrel{\circ}{\circ}$ & $\stackrel{\circ}{\circ}$ & $\bar{\gamma}$ & q \\
\hline 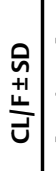 & $\mid \begin{array}{l}\overline{\underline{\underline{E}}} \\
\overrightarrow{\underline{\epsilon}} \\
\underline{\underline{\varepsilon}}\end{array}$ & 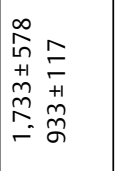 & $\begin{array}{l}\tilde{y} \\
y \\
+1 \\
D \\
上\end{array}$ & $\left|\begin{array}{c}O \\
\frac{1}{d} \\
+1 \\
\frac{1}{N}\end{array}\right|$ & $\mid \begin{array}{c}\infty \\
0 \\
+ \\
+1 \\
+ \\
0 \\
0\end{array}$ & $\begin{array}{l}\stackrel{n}{2} \\
+1 \\
\text { N } \\
\text { ஸे }\end{array}$ & $\begin{array}{l}0 \\
\infty \\
+1 \\
\stackrel{+}{0} \\
N\end{array}$ & $\mid \begin{array}{c}c \\
\infty \\
+1 \\
0 \\
\sim \\
\sim\end{array}$ & $\mid$\begin{tabular}{c}
7 \\
\multirow{2}{*}{} \\
+1 \\
0 \\
$\sigma$ \\
$\sigma$
\end{tabular} & 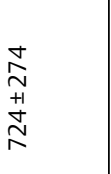 & $\begin{array}{l}0 \\
i \\
+1 \\
\infty \\
\infty \\
n\end{array}$ & 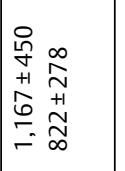 & 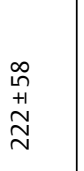 & 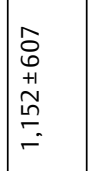 & 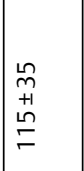 & 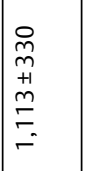 & 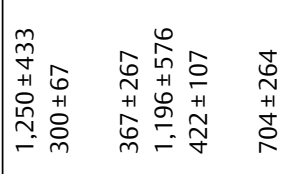 \\
\hline 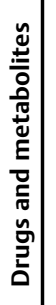 & & 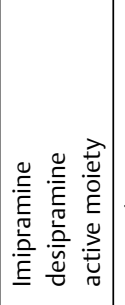 & 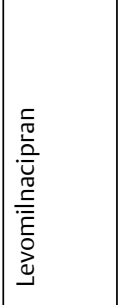 & 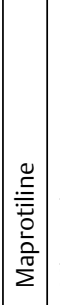 & 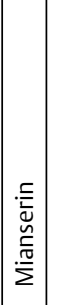 & 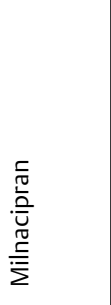 & 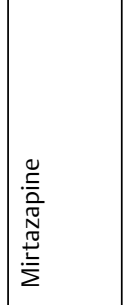 & 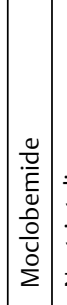 & 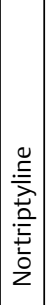 & 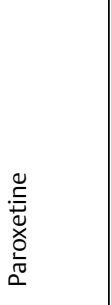 & 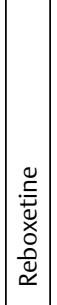 & 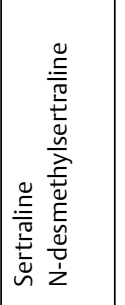 & 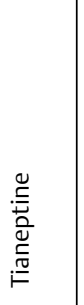 & 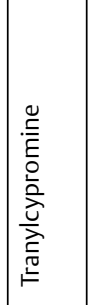 & 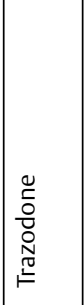 & 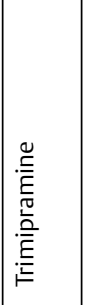 & 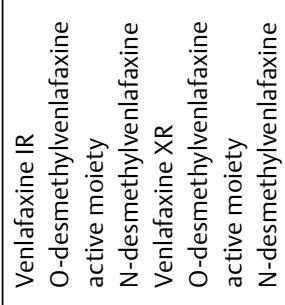 \\
\hline
\end{tabular}




\begin{tabular}{|c|c|c|c|c|c|c|c|c|c|c|c|c|c|c|c|c|c|c|c|c|c|c|}
\hline 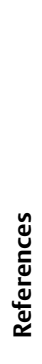 & & & 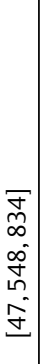 & & 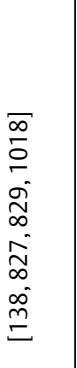 & 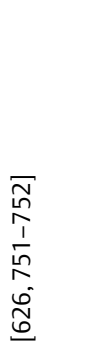 & $\mid \begin{array}{l}\bar{N} \\
\frac{1}{m} \\
\hat{n} \\
\underline{\Xi} \\
=\end{array}$ & $\mid \begin{array}{l}\infty \\
0 \\
0 \\
=\end{array}$ & $\overline{\mathcal{F}}$ & 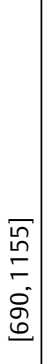 & 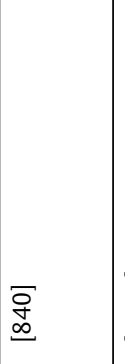 & $\mid \begin{array}{c}\infty \\
m \\
\stackrel{m}{=}\end{array}$ & $\begin{array}{l} \\
\overline{2} \\
\vdots \\
\tilde{\sigma} \\
\underline{\omega}\end{array}$ & 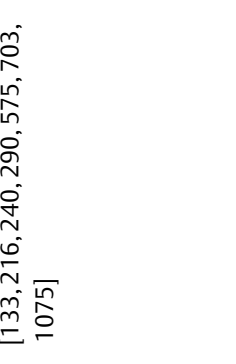 & 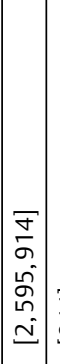 & 守 & 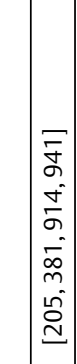 & 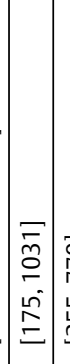 & 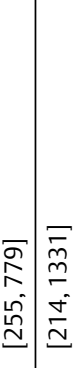 & 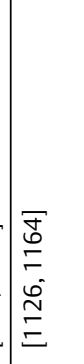 & 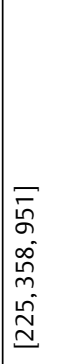 & $\stackrel{\substack{n \\
\Xi}}{=}$ \\
\hline 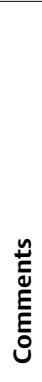 & & 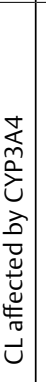 & 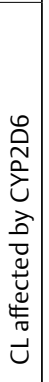 & & 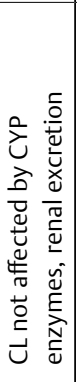 & 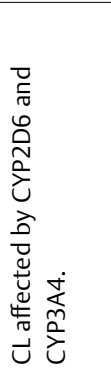 & 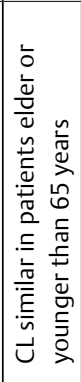 & & 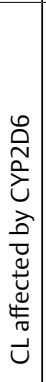 & & 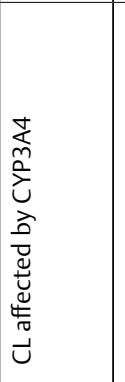 & & & 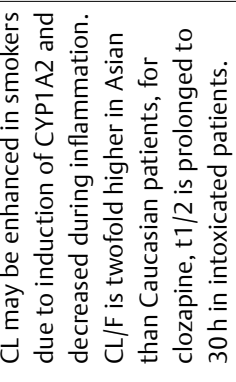 & 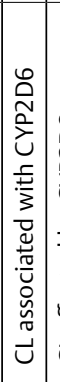 & 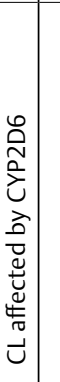 & 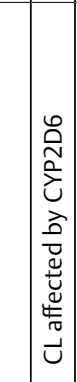 & 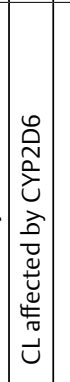 & 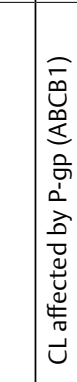 & 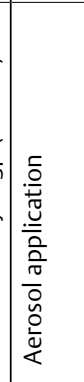 & 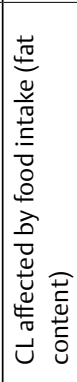 & \\
\hline & 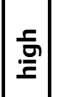 & ب़ِ & $\stackrel{\infty}{\stackrel{2}{\sim}}$ & & $\begin{array}{l}\infty \\
\infty \\
0\end{array}$ & 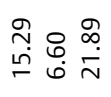 & F⿱ & $\mid \begin{array}{l}L \\
0 \\
0 \\
0\end{array}$ & $\begin{array}{l}\tilde{N} \\
\tilde{\gamma}\end{array}$ & 竞 & 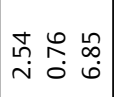 & $\stackrel{\circ}{\check{-}}$ & \begin{tabular}{c|c}
0 & \\
$m$ & 1 \\
$o$ &
\end{tabular} & 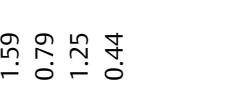 & 竎 & : & 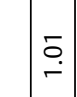 & $\begin{array}{l}\pi \\
0 \\
0\end{array}$ & $\stackrel{\substack{f \\
:}}{\stackrel{?}{\leftarrow}}$ & $\stackrel{\infty}{\stackrel{\infty}{*} \stackrel{\sim}{\sim}} \underset{0}{0}$ & $\stackrel{m}{0}$ & $\begin{array}{l}\bar{n} \\
\tilde{O} \\
\end{array}$ \\
\hline & 宐 & $\begin{array}{c}\infty \\
\infty \\
0\end{array}$ & $\begin{array}{l}\text { Jे } \\
\text { o. }\end{array}$ & & f. & 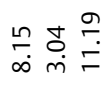 & oे & 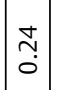 & 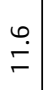 & 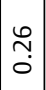 & 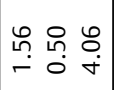 & $\mid \begin{array}{l}0 \\
\text { L } \\
0\end{array}$ & 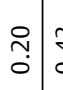 & 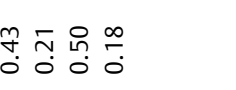 & शి. & $\begin{array}{l}n \\
0 \\
0\end{array}$ & 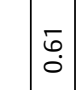 & 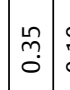 & \begin{tabular}{ll}
0 \\
\hdashline \\
0
\end{tabular} & 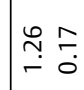 & o & $\begin{array}{l}0 \\
\\
0\end{array}$ \\
\hline 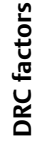 & 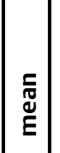 & 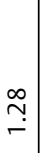 & 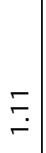 & & $\begin{array}{l}50 \\
0\end{array}$ & 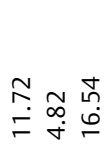 & $\stackrel{\stackrel{n}{n}}{\stackrel{0}{0}}$ & ơ & $\stackrel{\sim}{\grave{N}}$ & $\underset{\sim}{\tilde{\sigma}}$ & 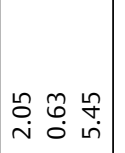 & \begin{tabular}{|c|}
$\infty$ \\
0 \\
0
\end{tabular} & 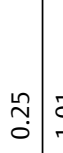 & 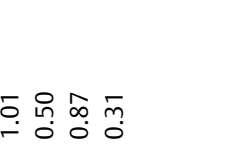 & $\begin{array}{c}\tilde{n} \\
\tilde{\sigma}\end{array} \mid$ & $\begin{array}{l}5 \\
0 \\
0\end{array}$ & $\mid \begin{array}{ll}-\infty \\
0 \\
0 \\
0\end{array}$ & $\left|\begin{array}{c}n \\
\stackrel{n}{0}\end{array}\right|$ & 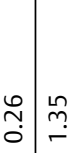 & 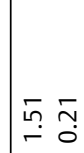 & $\overline{5}$ & $\stackrel{\infty}{\circ}$ \\
\hline $\overrightarrow{4}$ & $\Xi$ & $\stackrel{\Delta}{\sim}$ & $\stackrel{ \pm}{\sim}$ & & $\stackrel{d}{d}$ & $\stackrel{ \pm}{\sim}$ & $\simeq$ & $\simeq$ & 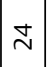 & $\simeq$ & $\stackrel{d}{d}$ & $\stackrel{\sim}{\sim}$ & $\simeq ?$ & $\simeq \stackrel{\Xi}{\sim}$ & $\simeq$ & $\simeq$ & $\simeq$ & $\simeq:$ & $\simeq \simeq$ & $\nabla \stackrel{\sim}{N}$ & $\stackrel{\Delta}{\sim}$ & $\simeq$ \\
\hline$\frac{\pi}{2}$ & $\Xi$ & $\tilde{m}$ & $\&$ & & $\stackrel{\bullet}{-}$ & ং む & $\stackrel{ \pm}{\sim}$ & 0 & के & $\stackrel{\sim}{\sim}$ & $\forall \begin{array}{l}\forall \\
\dot{m}\end{array}$ & $\stackrel{\circ}{m}$ & $\circ ?$ & $\simeq \quad \infty$ & $\stackrel{m}{m}$ & $\stackrel{6}{\circ}$ & $\stackrel{\infty}{-}$ & $\stackrel{\infty}{\sim}$ & $\stackrel{\infty}{\sim} \mid \infty$ & 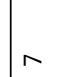 & $\stackrel{\infty}{\leftarrow}$ & in \\
\hline\llcorner & ஓ & 尺 & $\infty$ & & 오 & 8 & $\stackrel{n}{m}$ & in & เू & $\stackrel{m}{.}$ & 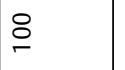 & $\stackrel{m}{.}$ & in & ㅇํㄴ & in & $\stackrel{n}{m}$ & 8 & 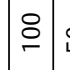 & in $\stackrel{m}{m}$ & m & 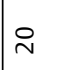 & 8 \\
\hline 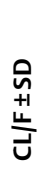 & $\left|\begin{array}{l}\overline{\underline{\underline{E}}} \\
\overrightarrow{\underline{\underline{E}}} \\
\underline{\underline{\underline{E}}}\end{array}\right|$ & 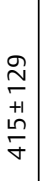 & 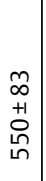 & & 茫 & 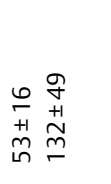 & 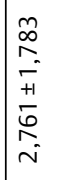 & $\left|\begin{array}{l}m \\
i n \\
+1 \\
0 \\
0 \\
\stackrel{n}{-} \\
-\end{array}\right|$ & $\begin{array}{l}\frac{}{m} \\
+1 \\
\tilde{N}\end{array}$ & $\mid \begin{array}{l}0 \\
0 \\
+1 \\
0 \\
0 \\
0 \\
-1 \\
-\end{array}$ & 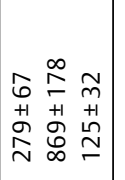 & 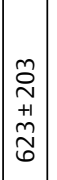 & 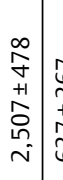 & 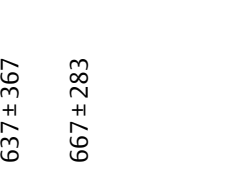 & 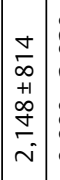 & 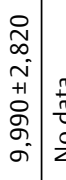 & 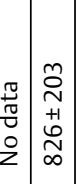 & 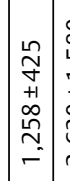 & 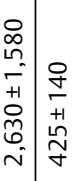 & $\mid \begin{array}{l}\infty \\
0 \\
+1 \\
++1 \\
0 \\
\infty \\
\infty\end{array}$ & 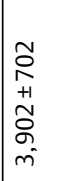 & 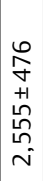 \\
\hline 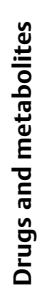 & & 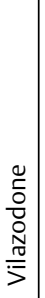 & 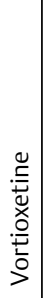 & 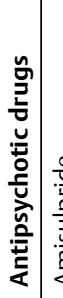 & 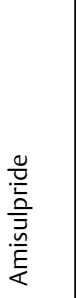 & 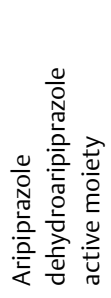 & 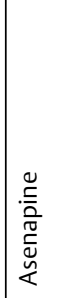 & 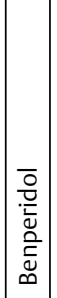 & 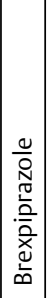 & 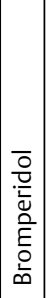 & 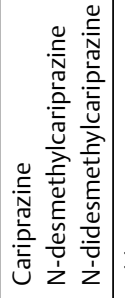 & 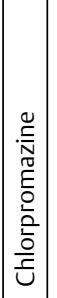 & 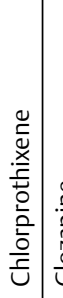 & 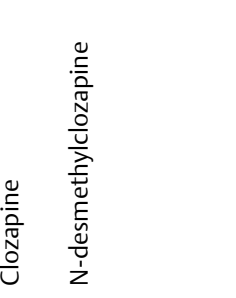 & 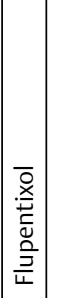 & 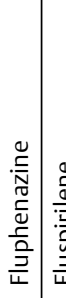 & 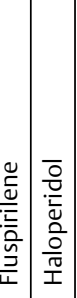 & 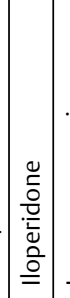 & 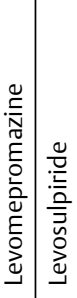 & 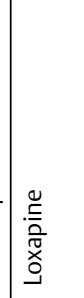 & 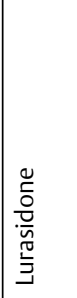 & 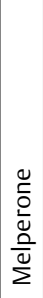 \\
\hline
\end{tabular}




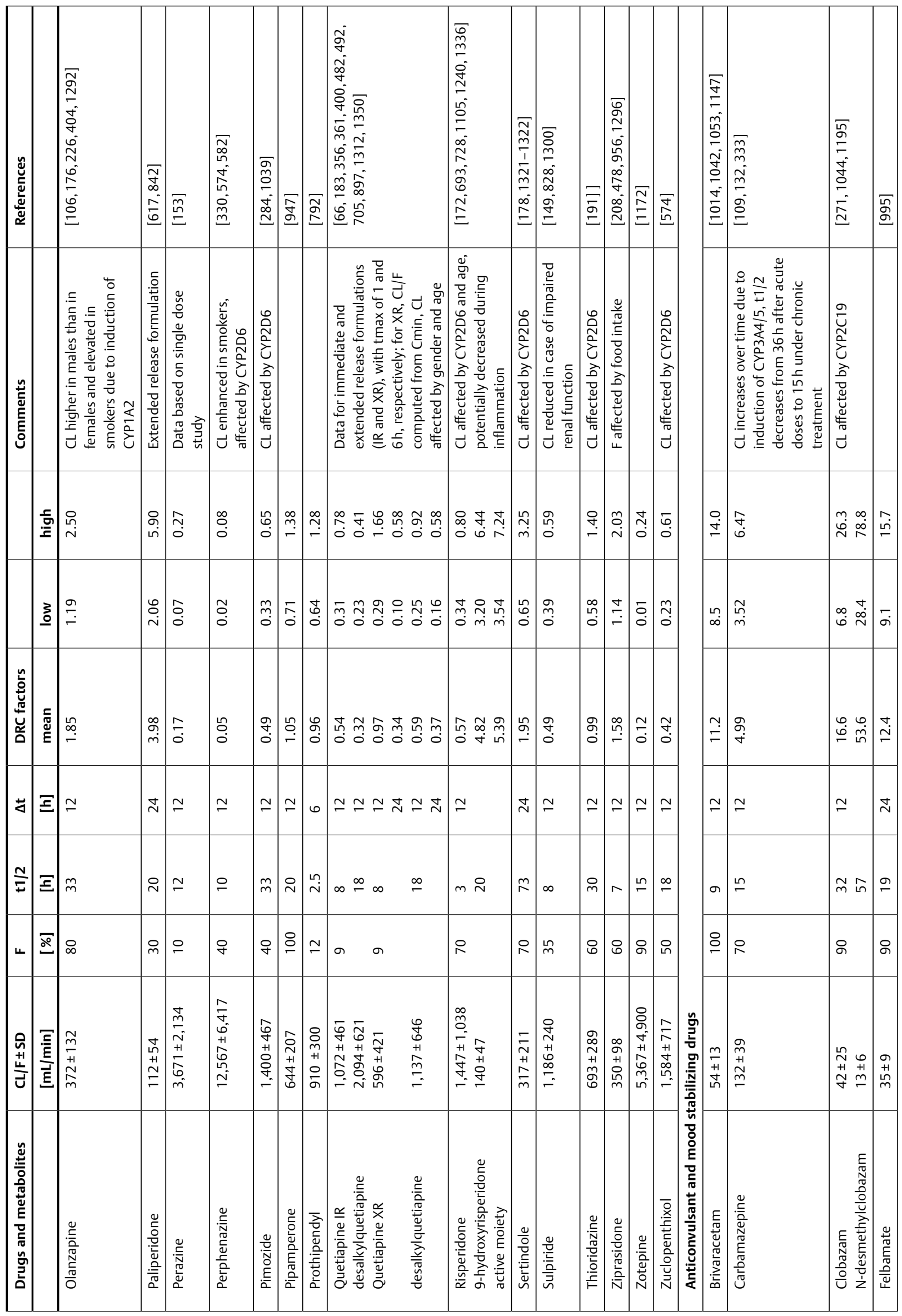




\begin{tabular}{|c|c|c|c|c|c|c|c|c|c|c|c|c|c|c|c|c|}
\hline 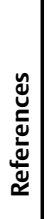 & & 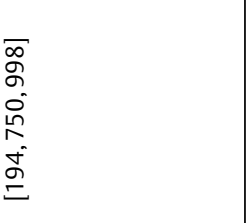 & $\begin{array}{l}\bar{N} \\
\hat{\sigma} \\
o \\
\tilde{n} \\
\end{array}$ & 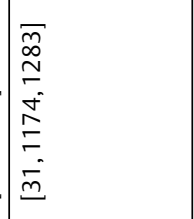 & 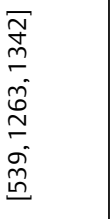 & 离 & $\begin{array}{l} \\
\tilde{\sigma} \\
\tilde{\sigma} \\
\end{array}$ & $\mid \begin{array}{l}\bar{\sigma} \\
\bar{\Omega} \\
\hat{n} \\
\tilde{D} \\
\tilde{n} \\
\end{array}$ & 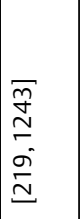 & & 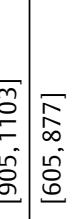 & $\begin{array}{l}\bar{m} \\
\infty \\
\infty \\
\infty \\
\infty \\
\omega \\
\end{array}$ & 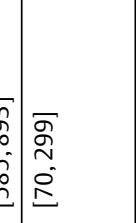 & $\begin{array}{l}\infty \\
\infty \\
\infty\end{array}$ & $\mid \begin{array}{l}\overline{\widetilde{N}} \\
\tilde{\Xi} \\
\end{array}$ & $\begin{array}{l}\tilde{n} \\
\infty \\
\infty\end{array}$ \\
\hline 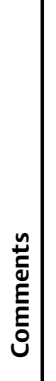 & & 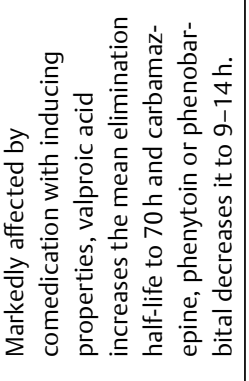 & & 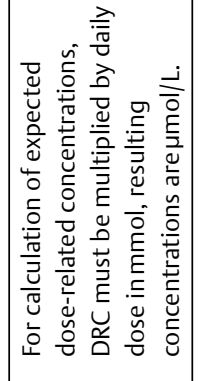 & & 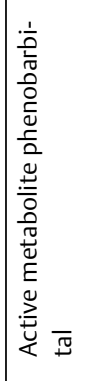 & & & 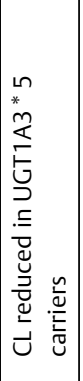 & 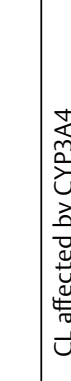 & 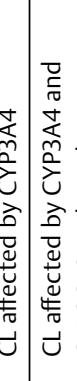 & 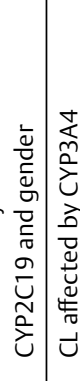 & 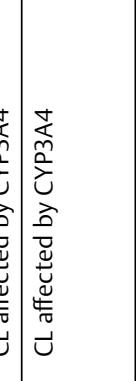 & 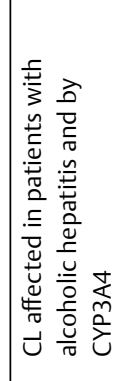 & 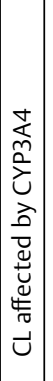 & 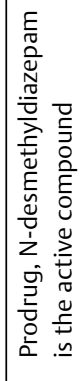 \\
\hline & 昰 & $\begin{array}{l}\tilde{I} \\
\dot{+}\end{array}$ & $\mid \begin{array}{c}\stackrel{9}{0} \\
\tilde{\rho} \\
\stackrel{0}{0}\end{array}$ & $\begin{array}{ll}0 & 0 \\
\dot{m} & \stackrel{0}{\sim}\end{array}$ & $\stackrel{\dot{0}}{\circ} \stackrel{-}{\infty}$ & $\stackrel{\nabla}{\dot{\sim}}$ & $\begin{array}{l}\infty \\
\dot{g} \\
\stackrel{g}{ }\end{array}$ & $\mid \begin{array}{l}\infty \\
\dot{\sim} \\
\dot{\sim}\end{array}$ & 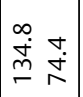 & & 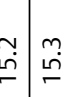 & $\stackrel{i}{i}$ & $\begin{array}{lll}\bar{v} & 0 & 0 \\
0 & 0 & 0 \\
0 & 0 & 0\end{array} \mid$ & $\begin{array}{l}\dot{0} \\
\text { in }\end{array}$ & $\mid \begin{array}{c}\infty \\
\stackrel{+}{=} \\
\end{array}$ & $\stackrel{\stackrel{n}{r}}{\sigma}$ \\
\hline & \begin{tabular}{l|l}
3 & 5 \\
0 & 4 \\
6
\end{tabular} & นึ. & 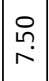 & 官 & $\overline{\check{O}} \overline{\dot{I}}$ & $\hat{m}$ & ָे & $\stackrel{-}{\infty}$ & 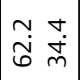 & & $\begin{array}{r}\sigma \\
\sigma\end{array}$ & $\stackrel{\tilde{\sigma}}{-}$ & : & $\overline{\dot{m}}$ & 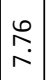 & $\stackrel{\substack{\infty \\
\infty}}{\sim}$ \\
\hline 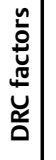 & 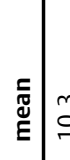 & $\stackrel{m}{o}$ & $\mid \begin{array}{c} \pm \\
\infty \\
\infty\end{array}$ & 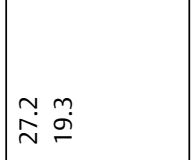 & 举 & $\stackrel{\stackrel{n}{\sim}}{\stackrel{1}{=}}$ & $\begin{array}{l}0 \\
\dot{\infty}\end{array}$ & $\stackrel{\grave{\sim}}{\grave{\sim}}$ & 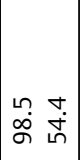 & & 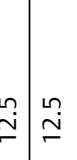 & $\stackrel{9}{i}$ & 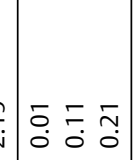 & $\stackrel{q}{\dot{f}}$ & \begin{tabular}{l}
\multirow{\sigma}{*}{} \\
$\sigma$
\end{tabular} & $\begin{array}{l}\circ \\
\stackrel{\infty}{m} \\
\stackrel{m}{m}\end{array}$ \\
\hline+ & $\Xi \lesssim$ & $\stackrel{\Delta}{\sim}$ & $\simeq$ & $\simeq \stackrel{\nabla}{\sim}$ & $\simeq$ & $\simeq$ & $\stackrel{\grave{N}}{\sim}$ & $\simeq$ & $\simeq \stackrel{ \pm}{\sim}$ & & 은 & $\stackrel{\circ}{\circ}$ & 20 & $\circ$ & 은 & 웅 \\
\hline$\frac{N}{E}$ & $\Xi$ & $\Xi$ & $r$ & $\stackrel{\searrow}{\sim}$ & $\sim a$ & i & $\infty$ & $\infty$ & 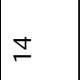 & & $m \approx$ & in & $m$ in 6 & $\approx$ & $\stackrel{\Perp}{\sim}$ & $\sim n$ \\
\hline$\leftarrow$ & ๖ & $\stackrel{8}{\circ}$ & 2 & $\stackrel{8}{\circ}$ & $\stackrel{\circ}{\circ}$ & 8 & $\triangleright$ & 8 & $\stackrel{8}{1}$ & & $\begin{array}{lll}\infty & \infty \\
\infty & \infty\end{array}$ & 尺 & $?$ & $\stackrel{8}{1}$ & $\infty$ & $m$ \\
\hline 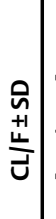 & 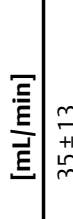 & 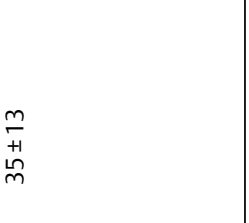 & $\begin{array}{c}0 \\
++1 \\
\stackrel{+}{\sigma}\end{array}$ & 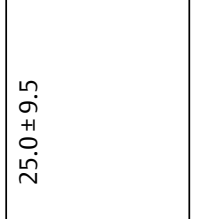 & 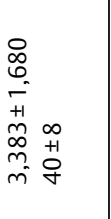 & \begin{tabular}{|l}
$n$ \\
$\infty$ \\
$\infty$ \\
+1 \\
$i n$ \\
$\infty$ \\
$\infty$ \\
$m$
\end{tabular} & $\begin{array}{l}\hat{m} \\
\dot{m} \\
+ \\
\dot{\omega} \\
\dot{\omega}\end{array}$ & 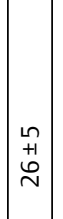 & 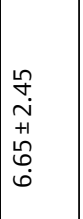 & 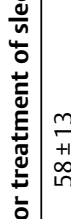 & 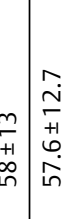 & $\begin{array}{l}\stackrel{N}{\stackrel{1}{*}} \\
\stackrel{+1}{\sim} \\
\stackrel{N}{N}\end{array}$ & 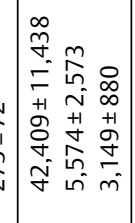 & $\begin{array}{l}\bar{m} \\
+1 \\
+\dot{\varphi} \\
\dot{\varphi}\end{array}$ & 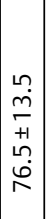 & $\begin{array}{l}\text { n } \\
+1 \\
+ \\
\sigma\end{array}$ \\
\hline 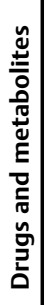 & & 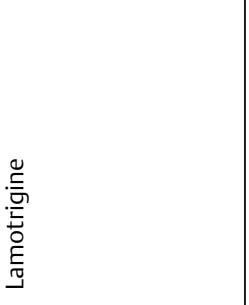 & 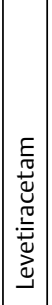 & 至 & 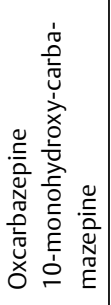 & 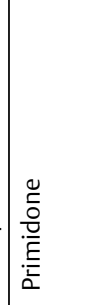 & 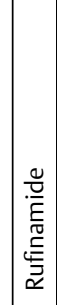 & 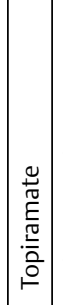 & 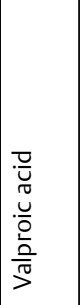 & 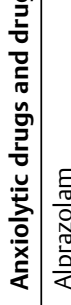 & 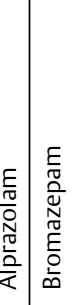 & 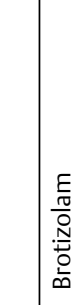 & 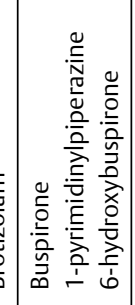 & 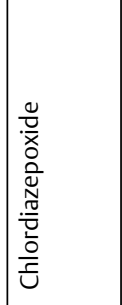 & 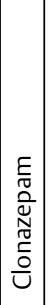 & 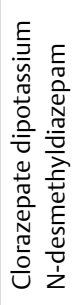 \\
\hline
\end{tabular}




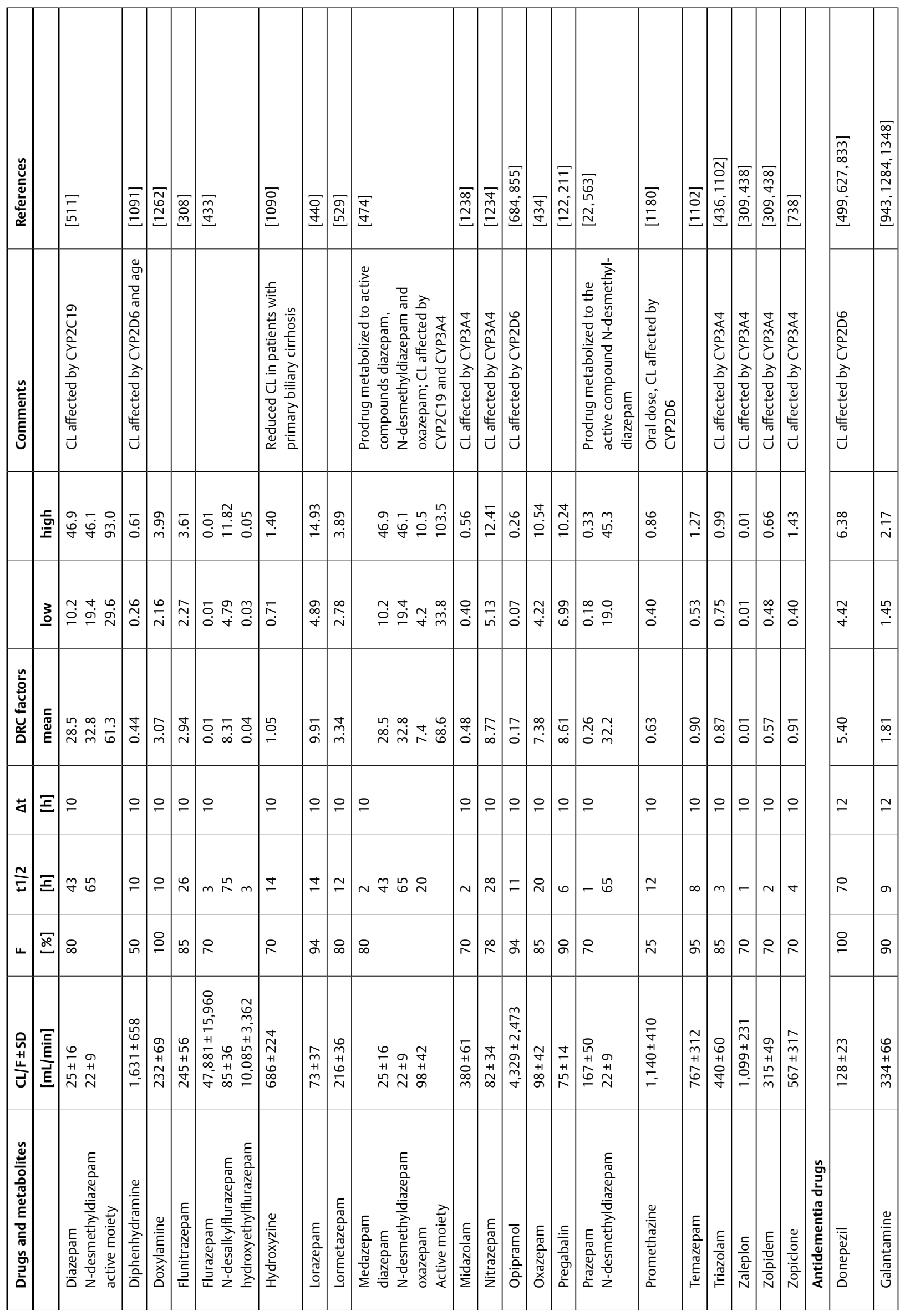




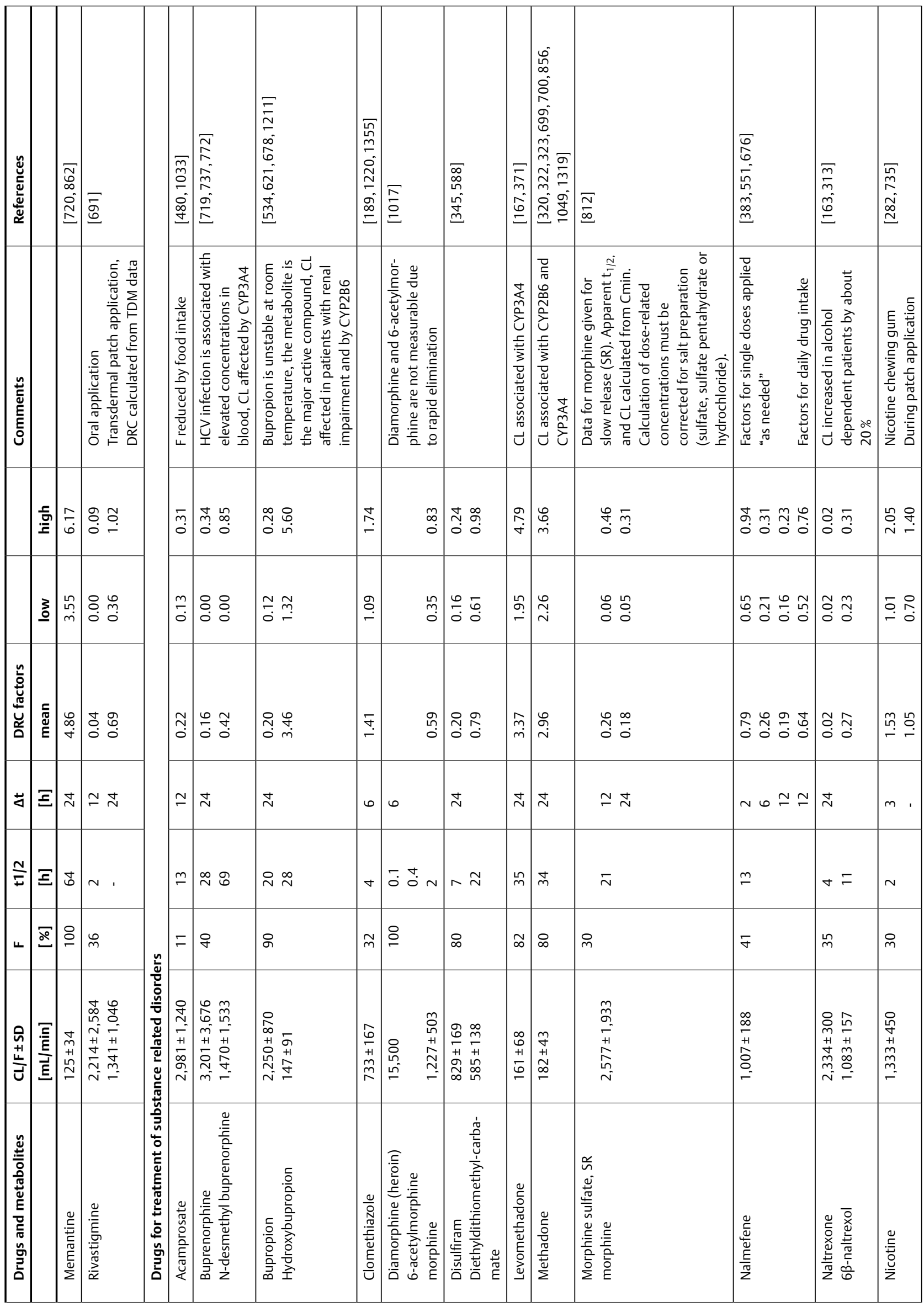




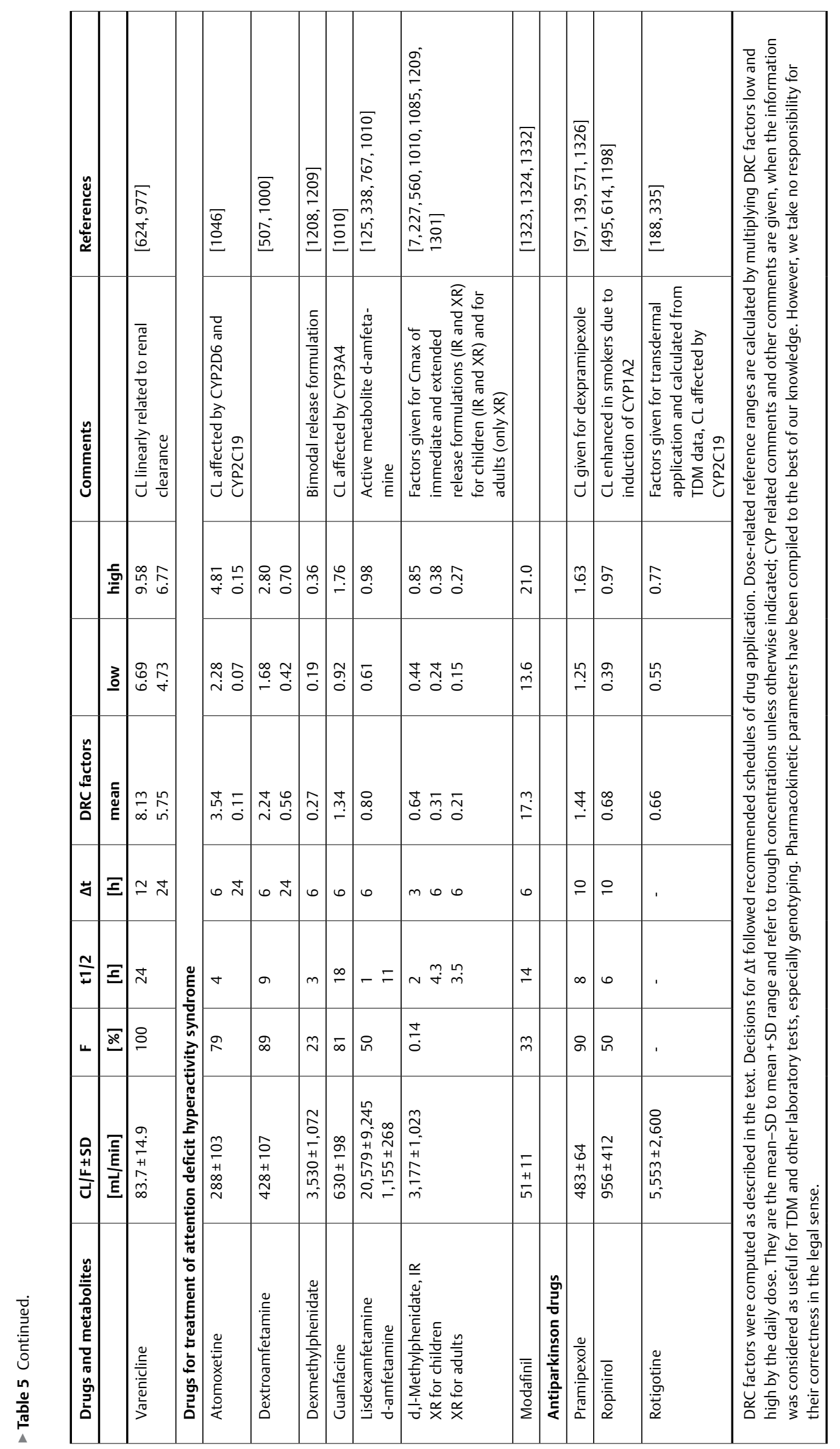


When $\Delta \mathrm{t}$ is different from values listed in $>$ Table $\mathbf{5}$, an expected drug concentration can be computed by Eq. (2) for any time point during the postabsorptive phase (time from maximal drug concentration).

Derived from the original concept of the dose-related reference range for average drug concentrations [471], the dose-related reference range is now defined as a $\mathrm{Cmin}$ range that can be calculated from the prescribed dosage and pharmacokinetic parameters.

\section{Definition}

The "dose-related reference range" reported in the present guidelines is defined as the mean-SD to mean + SD range of the trough concentration of a drug under steady-state conditions. The mean \pm SD includes $68 \%$ of a population of "normal" patients who ingested their medication, are aged 18-65 years, with a body weight of $70 \mathrm{~kg}$ and without pharmacokinetically relevant comorbidity, comedication or genetic abnormalities in drug metabolism. Dose-related reference ranges are obtained by multiplying DRC factors low and high of $>$ Table 5 by the daily dose.

The dose-related reference range is a discriminating reference range to identify patients with abnormal drug concentrations in blood. When practicing TDM, measured drug concentrations reported by a TDM laboratory should be compared with computed theoretical values by using data of $>$ Table 5 in these guidelines (see also cases below). When a patient's drug concentration is within the expected dose-related range, the concentration can be considered as "normal", i. e. the concentration is in accordance with the prescribed dose. Concentrations above or below the expected range are signals that indicate potential abnormalities such as partial non-adherence, drug-drug interactions, genetic polymorphisms of drug metabolizing enzymes or diseases of organs involved in drug elimination. Based on own experiences, abnormalities are assumed for about $1 / 3$ of the patients. Therefore, the mean \pm SD range $(68 \%$ of the patients) was considered as the range that is expected in "normal" patients. The validity of this assumption, however, still needs to be confirmed by studies. In case of observed abnormalities, suggested reasons should be explained in the clinical pharmacological TDM comment (see below) and causes should be clarified.

\subsection{Concentration to dose ratio}

The ratio of drug concentration to dose (Cmin/D, usually abbreviated as C/D) is a further parameter to analyse pharmacokinetic abnormalities [271, 500]. C/D can be easily calculated from TDM data by dividing the drug trough steady-state concentration by the dose that the patient is taking. C/D ratios are inversely related to total clearance [271, 292]. A high C/D ratio indicates slow and a low C/D ratio rapid drug clearance.

C/D ratios were used to detect drug-drug interactions by comparing different patient groups (e.g., [169, 586, 918, 1054, 1055]). Jerling and co-workers measured intraindividual C/D ratios of amitriptyline and nortriptyline and found interacting effects of levome- promazine, perphenazine and carbamazepine by showing that on and off of concomitant drugs corroborated previous C/D results [573]. Repeated measurement of C/D ratios in the same patients also helps to detect partial non-adherence to medication as it was shown for clozapine [1142]. Intraindividual variability of C/D should be below $20 \%$. Variability exceeding $20 \%$ points to adherence problems or pharmacokinetic alterations due to drug-drug, drug-food or drugdisease interactions.

The $C / D$ ratio can also be used to estimate the dose required to achieve a desired target concentration of the drug in blood [48]. Given, for example, that a C/D ratio of $0.5(\mathrm{ng} / \mathrm{mL}) / \mathrm{mg}$ was determined and the drug's therapeutic reference range is $30-100 \mathrm{ng} /$ $\mathrm{mL}$, a daily dose of $60(=30 / 0.5) \mathrm{mg}$ is required to reach $30 \mathrm{ng} / \mathrm{mL}$ and $200(=100 / 0.5) \mathrm{mg}$ to reach $100 \mathrm{ng} / \mathrm{mL}$.

\subsection{Metabolite to parent compound ratios}

Biotransformation of neuropsychiatric drugs by phase 1 enzymes may lead to metabolites with similar or different pharmacodynamic properties as their respective parent compounds. Examples for metabolites with similar properties are nortriptyline (parent compound: amitriptyline), $\mathrm{N}$-desmethyldoxepin (parent compound: doxepin), desipramine (parent compound: imipramine), norfluoxetine (parent compound: fluoxetine), O-desmethylvenlafaxine (parent compound: venlafaxine), or 9-hydroxyrisperidone (parent compound: risperidone). For these drugs, the sum of the concentrations of parent compound and active metabolite, i. e., the active moiety, is relevant for TDM-guided dosing. Examples for metabolites with different pharmacodynamic characteristics compared with their parent drugs are carbamazepine-10,11-epoxide (more toxic than carbamazepine), $\mathrm{N}$-desmethylclomipramine (noradrenergic activity: parent compound: clomipramine), N-desmethylclozapine (cholinomimetic activity; parent compound: clozapine) or $\mathrm{N}$-desalkylquetiapine (noradrenergic activity; parent compound: quetiapine). Major metabolites of olanzapine, sertraline or citalopram seem unlikely to contribute to the parent drugs' efficacy or tolerability. It can be argued that the monitoring of metabolites is useless when metabolites are devoid of pharmacodynamic activity. From a pharmacokinetic perspective, however, determination of active and non-active metabolites can be informative. The metabolite to parent compound ratio (MPR) is a direct measure of metabolizing enzyme(s) activity in vivo $[265,580,602,693,759,760$, $1074]$. When a distinct CYP isoenzyme is predominantly involved in a phase 1 reaction, MPR even reflects the phenotype of this CYP enzyme ( $\triangleright$ Table 6). MPR allows identification of abnormal metabolism caused by pharmacokinetic interactions or genetic abnormalities. For venlafaxine and risperidone, a low MPR is indicative for a poor metabolizer (PM) genotype of CYP2D6. PM genotypes can be differentiated from extensive metabolizer (EM) genotypes with a sensitivity of $91 \%$ [759]. A high MPR points to enhanced enzymatic activity and thus indicates an ultrarapid metabolizer (UM) status. Moreover, enzyme inducing effects, e. g., of CYP1A2 by cigarette smoke, can be identified by an MPR enhancing effect. For sertraline, it has been shown how to use MPR of N-desmethylsertraline to sertraline for identification of patients' adherence to the prescribed medication $[173,985,1023]$. 


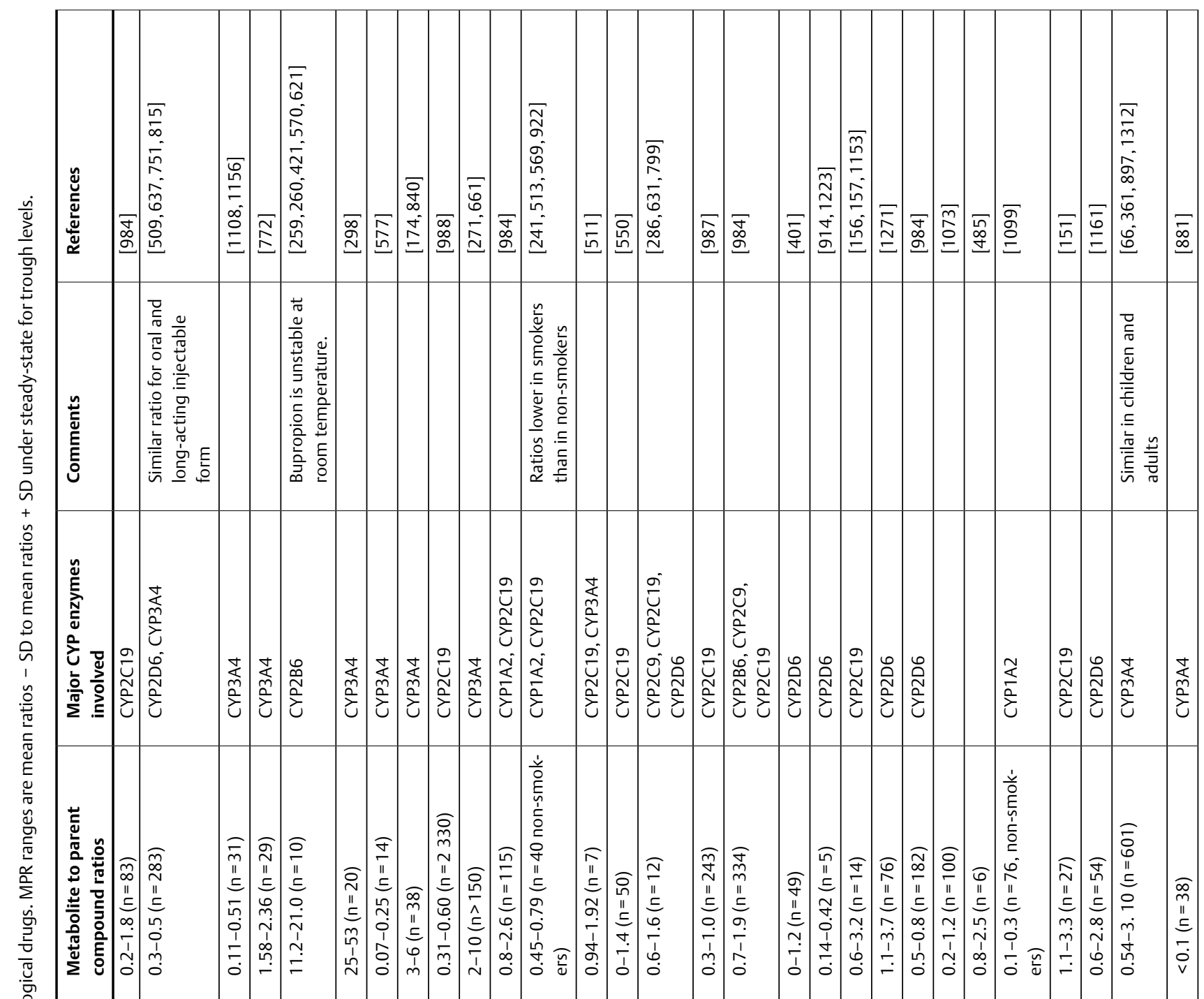

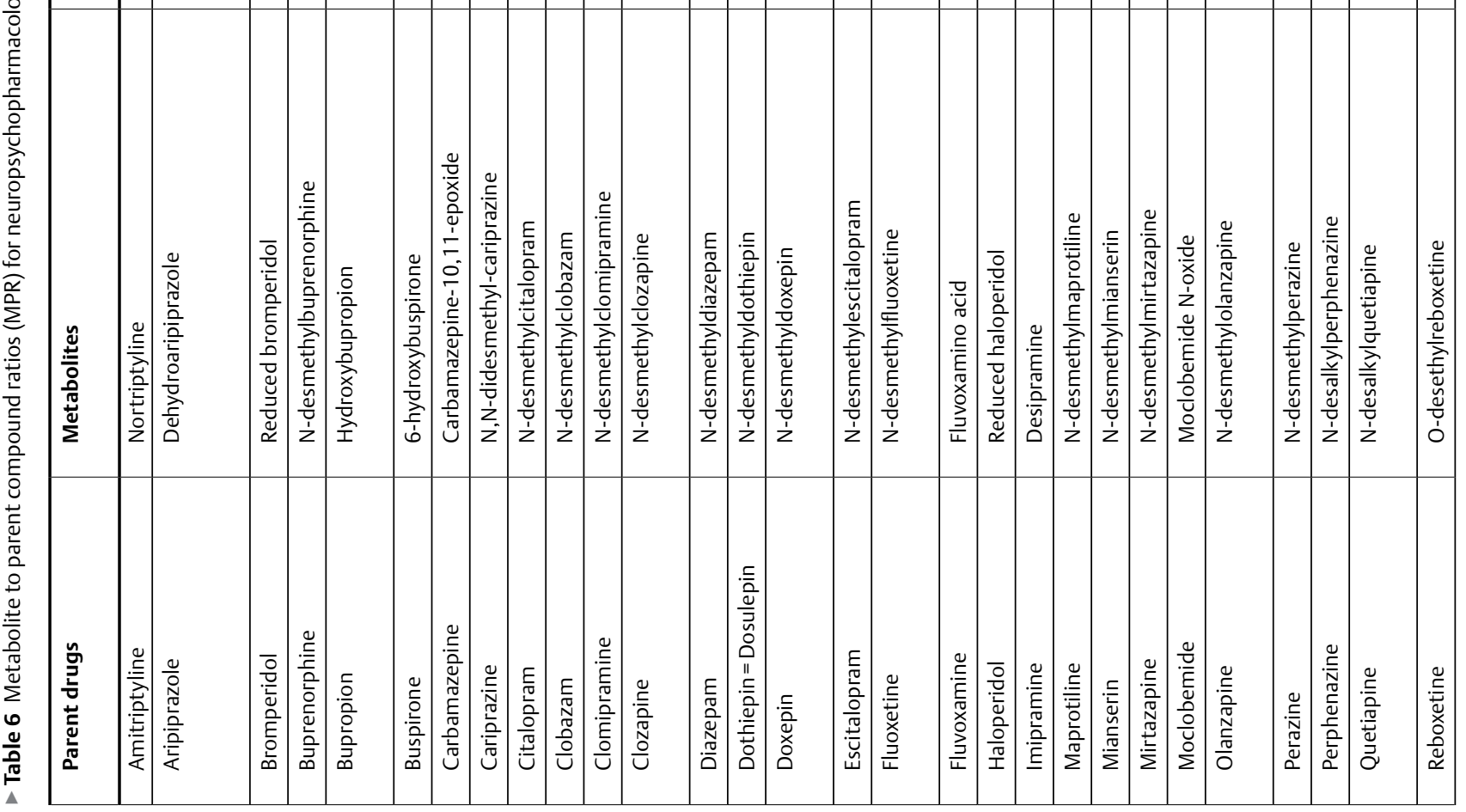




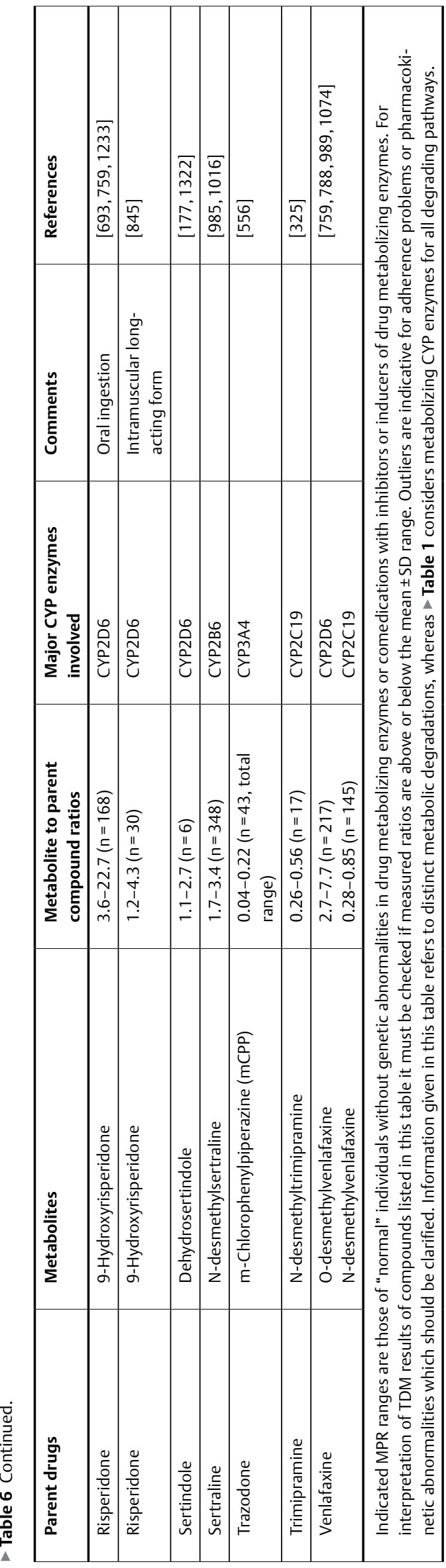

When using MPR to characterize a patient's metabolic phenotype, confounding factors must be well controlled to avoid false conclusions. Especially the correct timing of blood sampling is essential when parent drug and metabolite have different elimination half-lives.

The validity of MPR to predict CYP gene variants has been proven for risperidone and venlafaxine [602, 760, 1074, 580, 759]. For risperidone and its metabolite 9-hydroxyrisperidone, the cut-off MPR for EM and PM of CYP2D6 was 1.0. Its sensitivity was $91 \%$, its specificity $86 \%$ and its positive predictive value $35 \%$, while the negative predictive value was $99 \%$ [759]. Similar results were found for venlafaxine and its major metabolite $\mathrm{O}$-desmethylvenlafaxine. The cut-off MPR of 1.0 had a sensitivity of $93 \%$, a specificity of $86 \%$, a positive predictive value of $40 \%$ and a negative predictive value of $99 \%$ [759]. To discriminate UM and EM, the MPR value was less sensitive. Phenotypes of these genotypes overlap. Thereby it has to be considered that UM genotypes of CYP2D6 explains only $30 \%$ of UM phenotypes. Despite some limitations, we recommend to determine MPR for the characterization of the patient's metabolic phenotype.

\section{Definition}

The term "metabolic ratio" is used inconsistently in the literature, either as ratio of concentration of parent compound to metabolite or vice versa metabolite to parent compound. To avoid confusion, we use the term metabolite to parent compound ratio (MPR). MPR values shown in > Table 6 for 35 neuropsychiatric drugs are in vivo estimates of the enzymatic activities involved in the metabolism of the respective drugs. Assuming normal distribution, mean MPRs \pm standard deviation (SD) were calculated for standard dosages. Outliers of the mean \pm SD range may point to partial non-adherence or abnormalities in drug metabolism which should be clarified.

\subsection{Probe drug phenotyping}

The pharmacokinetic phenotype is measured by so-called 'probe drug' tests. They were introduced in the past when it was observed that the metabolism of drugs is genetically determined. This was found for a number of drugs like debrisoquine, mephenytoin, sparteine and also for the antidepressant drug nortriptyline [21]. Systematic research identified compounds that are preferably metabolized by distinct CYP enzymes. Using this knowledge, phenotyping tests were developed and validated with specific probe drugs, e. g., caffeine for CYP1A2, efavirenz for CYP2B6, losartan or tolbutamide for CYP2C9, omeprazole or mephenytoin for CYP2C19, dextromethorphan, debrisoquine or metoprolol for CYP2D6, midazolam or erythromycin for CYP3A4, and chloroxazone for CYP2E1 [218, 283, 343, 373, 425, 527, 533, 644, 722, 847, 1121, 1170]. Subjects ingest the probe drug, whenever possible in a pharmacodynamically ineffective dose, and concentrations of parent compound and metabolite formed by the indicator reaction are determined. Their concentrations or ratios of concentrations reflect the in vivo activity of the respective CYP enzyme. Progress in drug ana- 
- Table 7 Typical indications for measuring drug concentrations in blood of psychiatric or neurologic patients.

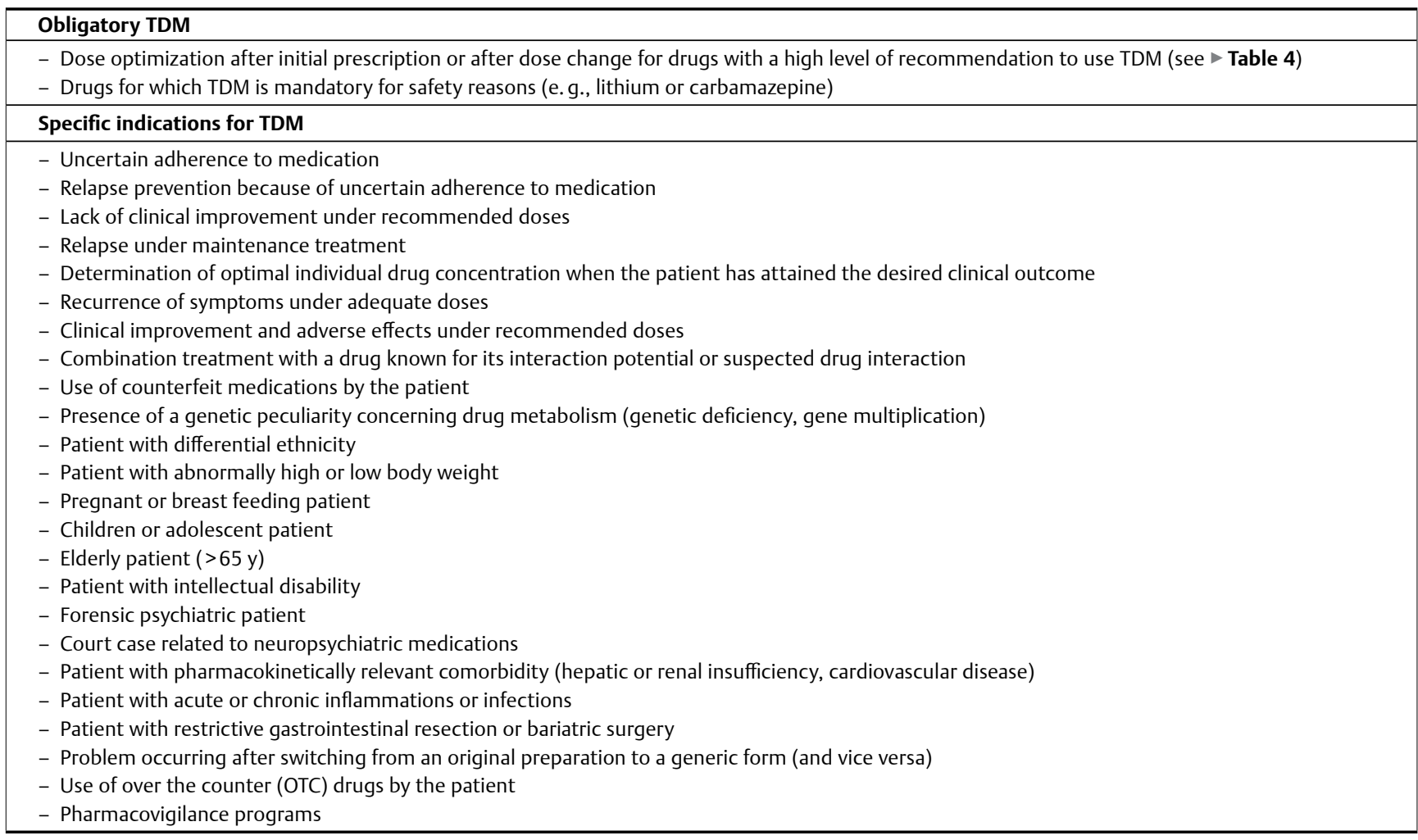

lysis by mass spectrometry enabled the use of cocktails containing six or more probe drugs. They allow quantifying the activity of several isoenzymes by a single test. One practical idea for probe drug phenotyping was to measure the optimal dose of an intended drug. Such assays, however, were not successful so far. Since only few drugs are metabolized by a single isoenzyme, it is difficult to compute the optimal dose based on phenotyping tests. It was found more appropriate to analyze the drug concentration of the prescribed drug, i. e. to use TDM for dose finding. Phenotyping tests, however, are well established for evaluation of pharmacokinetic interactions, preferentially during drug development. When evidence is given by in vitro data that a new drug has CYP inhibiting or inducing properties, a phenotyping test is recommended for clarification [370]. Moreover, phenotyping by probe drugs can be helpful as add-on for TDM. Using caffeine as probe drug, the inducing effect of smoke on CYP1A2 activity was characterized. It could be shown that the inducing effect disappears within four days after cessation of heavy smoking [342].

\subsection{Indications for measuring drug concentrations in blood}

- Table 7 presents a list of indications for TDM in psychiatry and neurology. The validity of these indications has to be examined and evaluated for each case individually. Similar to any diagnostic test, TDM should only be requested when there is evidence that the result will provide an answer to a well-defined question.
For drugs with established therapeutic reference ranges or with a narrow therapeutic index, it makes sense to measure drug concentrations in blood for dose titration after initial prescription or after dose change. Even without a specific problem, there is sufficient evidence that TDM has beneficial effects for patients treated with the following drugs: lithium, tricyclic antidepressants, several antipsychotics or anticonvulsants ( $\vee$ Table 4 ). For lithium, TDM is even mandatory for safety reasons.

Problems with adherence (non-adherence, partial adherence), a politically more correct term than compliance, since 'adherence' presupposes the patient to be a hierarchically equal partner in therapeutic decision making [49], are common and costly in pharmacotherapy. On average, $50 \%$ of medications for chronic diseases in general are not taken as prescribed [1356]. In studies on patients with schizophrenia $[90,603]$ and in patients with depression or bipolar disorder, non-adherence ranged from 10 to $69 \%$ $[264,716,795,1345]$. In a large sample of patients with dementia who were treated with choline esterase inhibitors, it was found that $34 \%$ were adherent within an observation period of 12 months [473]. Incomplete or total non-adherence impairs the effectiveness of treatment. According to a report of the World Health Organization [1325] it is suggested that improvement of adherence may have a far greater impact on the health of the population than any improvement in specific medical treatments. Methods used to measure adherence include pill-counting, addition of colouring agents detectable in urine, examining case-note recordings, interviewing patients or noting the attending physicians' clinical judgment about adherence $[14,612,1034,1246,1247,1286]$. Studies 
have shown that clinicians cannot reliably predict their patients' adherence $[171,725,1034]$. Measuring drug concentrations in blood is advantageous compared to other methods, since it tells the prescribing physician whether the drug is in the body at a concentration that is potentially sufficient to provide the expected clinical response. In patients with epilepsy, drug concentration monitoring confirmed more often non-adherence than adequate seizure control. For antiepileptic drugs, subtherapeutic levels were found in most patients attending hospitals due to seizures [1138]. Deviations from the expected dose-related reference range ( $\vee$ Table 5 ) indicate whether the patient has taken his medication and/or is a rapid or poor metabolizer. Concomitant determination of metabolites is another approach to clarify drug adherence. For interpretation, however, possible interactions with co-medications exhibiting enzyme inhibiting or inducing properties must be considered ( $\triangleright$ Table 2, 3). Reis and coworkers $[985,986]$ analysed the adherence of patients who were treated with sertraline by repeated determination of serum drug concentrations of the parent compound and of the metabolite. Variations of the $\mathrm{N}$-desmethylsertraline/sertraline ratio were highly indicative of hidden and partial non-adherence. As reported above, this consensus gives metabolite to parent compound ratios for 35 neuropsychiatric drugs ( $\triangleright$ Table 6). By taking several blood samples per day and by calculating the observed and expected time dependent drug concentrations in blood, it can be differentiated if a low drug concentration is due to reduced bioavailability, enhanced degradation or poor adherence. Pharmacokinetic modelling of the expected time dependent drug and metabolite concentration in blood enables identification of different types of non-adherence [5, 584, 1141, 1192].

Relapse prevention is a major goal of maintenance treatment. Reduction of relapse rates by TDM is highly cost-effective, as relapses can lead to re-hospitalization [124, 658, 1142]. In schizophrenic patients, it has been shown that fluctuations of clozapine concentrations in blood are predictive for relapses [391, 1219]. TDM may thus reduce the risk of relapse or recurrence by increasing the doctor's alertness concerning the patient's adherence to the medication.

\section{Recommendation}

We recommend regular monitoring of drug concentrations in blood under maintenance therapy, at least every 3-6 months, to prevent relapses and re-hospitalizations. The frequency of TDM requests may be increased if the patient is suspected to be non-adherent to the medication or in case of changes of co-medications or of smoking that affect the pharmacokinetics of the prescribed drug.

When clinical improvement under recommended doses is insufficient and the drug is well tolerated, TDM will clarify whether the drug concentration is too low and whether it makes sense to increase the dose.

When adverse drug reactions coincide with clinical improvement under recommended doses, measurement of the drug concentration in blood may clarify if these reactions are related to ex- cessively high drug levels in the blood and if the dose can be decreased without loss of efficacy.

When combining compounds that are inhibitors or inducers of drug metabolizing enzymes ( $\vee$ Table 2,3 ) with a drug that is a substrate of the inhibited or induced enzyme ( $\triangleright$ Table 1 ), dosing should be guided by TDM to avoid loss of action, poor tolerability or intoxication due to a pharmacokinetic drug-drug interaction [364, 412, 1081, 1236]. Effects of smoking should be considered when patients are under therapy with a CYP1A2 substrate such as clozapine, duloxetine, mirtazapine, olanzapine, rasagiline or ropinirol ( $\triangleright$ Table 1$)$.

In patients exhibiting genetic abnormalities of drug metabolizing enzymes, it may be necessary to adapt doses or apply therapeutic alternatives. Kirchheiner (Stingl) and coworkers $[630,633,1145]$ calculated doses for PM or UM of CYP2D6 based on pharmacokinetic and pharmacodynamic findings. These dose adjustments on pharmacogenetic evidence has been further adopted by international consortia such as the Pharmacogenetic Clinical Implementation consortium (CPIC), and evidence based guidelines on how to adjust therapy in the case of pharmacogenetic variants have been issued for tricyclics and SSRIs [516]. However, even in the case of a confirmed abnormal CYP genotype, TDM is recommended, because most CYP isoenzymes are not substrate-specific and genotyping can only roughly predict to which extent the drug concentrations in blood may be changed in the individual patient [905, 906, 1136].

Any neuropsychopharmacotherapy of pregnant or breastfeeding women should assure that the blood concentration of the drug is held in the therapeutic reference range to minimize the risk of relapse on the mother's side and, at the same time, minimize risks associated with drug exposure of the fetus or the infant $[35,280,289]$. Renal clearance and the activity of the CYP isoenzymes 2A6, 2C9, 2D6 and 3A4, and uridine 5'-diphosphate glucuronosyltransferase (UGT) 1A4 and 2B7 are increased during pregnancy, whereas activities of CYP1A2 and CYP2C19, and N-acetyltransferase 2 (NAT2) decrease [532, 773, 903]. TDM in pregnant women and/or mothers should be carried out at least once per trimester and within $24 \mathrm{~h}$ after delivery $[103,681]$.

Many neuropsychiatric drugs are not approved for use in children or adolescents $[416,1308]$. To date, therapeutic reference ranges for most neuropsychopharmacological drugs are based upon studies performed in adults, and data about the correlation of concentration with therapeutic response or adverse drug reactions in the paediatric population are scarce $[327,1298]$. The relative lack of clinical trials and the resultant off-label use could lead to a higher risk of dosing errors and adverse drug reactions. Pharmacokinetics and pharmacodynamics change during development $[328,794,939,945,1230]$, suggesting that dosing regimens as well as possible clinical effects in minors cannot be extrapolated from the evidence obtained in adults. Increasing prescription numbers in paediatric patients contrast with these uncertainties about safety and efficacy [327], and heavy responsibility is imposed upon both physicians and caregivers. Under these conditions, TDM is strongly recommended to individualize drug treatment and optimize drug safety. In adolescents suffering from psychotic disorders, comorbid drug abuse is very common, and adherence to antipsychotic treatment is generally marginal [538]. Therefore, TDM is 
even highly recommended for these patients. The extrapolation of therapeutic reference ranges - which have been established in adult patients - to paediatric patients, especially to young children, has to be investigated for every single substance, as preliminary TDM studies in paediatric neuropsychiatry provided divergent results. Fortunately, however, several studies have demonstrated similar therapeutic reference ranges for children/adolescents and adults (e. g., sertraline [1177], aripiprazole [949, 1311], fluvoxamine [677]). For most substances, a high interindividual variability in drug concentrations after administration of the same dose was shown in children and adolescents. Similar to adult patients concentrations were broadly related to prescribed dosages $[56,57,240,654,1177,1185]$. Finally, there is evidence indicating the necessity for higher weight-normalized dosages to achieve the concentrations within the reference range for adults, or suggesting that reference ranges are different from those for adults for drugs like quetiapine [400], clozapine [1314] or risperidone [647].

However, the implementation of TDM in the paediatric population is more difficult than in adults because sampling procedures often are invasive and require the cooperation of the patient [939]. As described below in more detail, ongoing research investigates the suitability of alternative matrices, e. g., saliva, and more convenient sampling techniques (e. g., bloodspot) in routine TDM to minimize inconvenience and patient discomfort in paediatric patients [362].

Besides a plea for more clinical trials and more pharmacokinetic-pharmacodynamic studies in children and adolescents, active and standardized surveillance and follow-up (i. e., patient monitoring) of children and adolescents starting drug treatment is necessary. A registry that captures such observations, assessments, and measurements including TDM of many patients in a standardized way was established to generate pharmacovigilance data (evidence) on dosing regimens, serum concentrations, the effectiveness and tolerability of neuropsychiatric drugs under every day conditions by a TDM competence network for child and adolescent patients [see http://www.tdm-kjp.com]. This approach could minimize the risk of exposing paediatric patients to ineffective or less tolerable psychotropic drug treatments [399].

For elderly patients, TDM should be used [1212], since ageing involves progressive impairments of the functional reserve of multiple organs [731]. Especially renal excretion and liver function may decrease significantly $[628,651]$. Phase 1 reactions are more likely to be impaired than phase 2 reactions. Glomerular filtration, tubular reabsorption, and secretion change with age, and also weight and volume of distribution [1060]. Hepatic clearance can be reduced by up to $30 \%$, which is mainly explained by a reduced hepatic blood flow rather than by a decrease of the activity of metabolic enzymes. According to some authors [651], there are no important age-dependent changes in CYP isoenzyme activity, while others suspect a slight decrease in the activity of CYP2D6, but not of CYP2C and CYP3A [1060]. Elderly patients are frequently hypersensitive to medication, and frailty is a major problem. They are at an increased risk of homeostasis loss after stressful events and a decreased ability to recover a stable situation [164]. For example, the cholinergic system seems to be supersensitive in aged subjects $[695,908]$. Many psychotropic drugs such as clozapine, tricyclic antidepressants or paroxetine display anticholinergic activity. Their use may result in the occurrence of delirium, decrease of cognitive functions and other serious adverse drug reactions [212]. As shown for nortriptyline, its anticholinergic activity increases with increasing blood concentrations, and occurs even at therapeutic nortriptyline concentrations [212]. The increased risk for adverse drug reactions has prompted many authors to develop criteria for identification of potentially inappropriate medication use in elderly patients, e. g., the Beers criteria [32], the PRISCUS list $[304,537,1057]$, STOPP [393] and others [874, 875, 1022]. On the other hand, elderly patients are often undersupplied with potentially useful drugs, including antidepressants [209]. In addition, the abovementioned frailty increases the risk of comorbidities and therefore also the risk of polypharmacy, complicating pharmacotherapy in the elderly $[164,207]$. Finally, the off-label prescription of psychotropic drugs seems to be frequent in the elderly patient population [561, 1140]. Clearly, there are still insufficient data available on the usefulness of TDM of psychotropic drugs in the elderly. The consequence of this situation is the relative absence of published recommendations to carry out TDM in this population, in order to optimize treatments. "Monitoring" is frequently recommended, but it does generally not explicitly include TDM $[872,1123,1200]$.

In individuals with intellectual disabilities, second-generation antipsychotics are frequently used. Practical guidelines recommend TDM for these patients, at least when treated with risperidone or olanzapine [270]. For ethical and legal reasons, patients with intellectual disabilities are excluded from clinical trials, though many of them need medication. In these individuals, it may be difficult to differentiate between disease and drug induced reasons for symptom aggravation. TDM is recommended as an objective guide for the pharmacotherapy of these patients [270, 272, 494, 1062].

In patients with increased C-reactive protein (CRP) indicating inflammation or infection and under pharmacotherapy with clozapine or risperidone, TDM is recommended to minimize the risk of intoxications due to elevated drug concentrations [501].

For patients with substance use disorders and dependence syndromes, the available medications with proven efficacy are candidates for TDM [163, 396, 477, 496, 689]. Their drug concentrations are highly variable between individuals [163]. For substitution therapies with opioid agonists, overdoses may have fatal consequences [686]. Moreover, the rate of non-adherence is high. The kind of non-adherence of these patients, however, differs from other patients $[685,747,1358]$. Patients with substance use disorder usually accept their substitution medication. But they may have the impression that their dose is insufficient and therefore may consume higher doses than prescribed or add illegally acquired drugs. Other patients discontinue substituted medication. For opioid dependent patients, medical treatment was only effective when they were adherent [1291]. The opiate agonists, i. e., racemic methadone, R-(-)-methadone (levomethadone), buprenorphine with and without naloxone, and slow-release formulations of morphine are used orally for opioid maintenance treatment. In certain cases, i.v. diacetylmorphine (heroin) is administered. TDM is highly recommended for methadone or R-(-)-methadone, buprenorphine and probably also for slow-release formulations of morphine. Based on drug properties and patient characteristics, the usefulness of TDM was evaluated for treatment of alcohol addiction with drugs such 
as acamprosate, naltrexone or disulfiram and of opioid addiction with naltrexone for abstinence-oriented treatment [163]. TDM has the potential to enhance the moderate efficacy of these drugs and enable the detection of pharmacokinetic abnormalities due to gene variants of drug metabolizing enzymes or to drug-drug interactions [1183]. Because of the different kind of adherence in patients with substance related disorders one must be aware that not only decreased but also increased drug concentrations may occur.

In forensic psychiatric patients, medication is important to reduce both the risk of violence and aggressive behavior and the burden of psychiatric symptoms [41, 493, 824, 825, 1201]. To achieve these goals, adherence to medication, mostly consisting of antipsychotic drugs, is essential, since most forensic psychiatric patients disapprove of pharmacotherapy [824, 825]. Castberg and Spigset [184] analyzed data of a high security forensic unit and found higher prescribed doses in forensic patients than in a control group, whereas the dose-related concentrations were significantly lower for olanzapine but higher for quetiapine in the forensic patients. TDM is highly recommended for this group of patients especially when supervised as outpatients.

In court cases concerning the alleged adverse drug reactions of psychotropic drugs (for example, pathological gambling allegedly induced by dopamine D2/D3 receptor agonists), TDM is instrumental for the court-certified witness (i. e., expert court witness) for proving or disproving that the claimant actually took the medication and reached drug concentrations in blood that plausibly caused the alleged harm [1345]. It has been shown that $55 \%$ of the claimants for disability pensions who had been diagnosed with depression and had been prescribed antidepressants had no detectable antidepressants in their blood [398]. A further $11 \%$ had antidepressant levels that were close to zero and far below the lower limit of the orienting therapeutic reference range. Thus, a total of $66 \%$ of disability pension claimants whose cases went to court could not prove that they actually took their antidepressant medication as claimed [398]. This is of great consequence, as a sick person has to contribute unambiguously to his/her reconvalescence. Only in case of treatment failure, he/she is eligible for sickness benefits or disability payments. Thus, in the sample cited above, $66 \%$ of the claimants did not fulfil the criterion, inviting suspicion of public health insurance fraud.

For the indication "switching from an original preparation to a generic form or vice versa ", TDM should be used instead of watching and waiting if problems such as loss of efficacy or tolerability problems evolve [206, 242]. In a study which compared originator and generics in volunteers [206], the venlafaxine generic yielded $50 \%$ higher levels during the absorption phase than the originator. Consequently, the frequency of adverse drug reactions was increased. This was not the case for the citalopram originator and a citalopram generic. Generics may be different from each other up to approximately $45 \%$. It is allowed for any generic to have an AUC0-24 h or AUC0-infinite AND a Cmax that is within 80 to $125 \%$ of the originator $(125 \%-80 \%=45 \%)$. There may be an even larger difference during the initial absorption phase, while the AUCO-infinite and $\mathrm{Cmax}$ are still within the $80-125 \%$ limit.

Other indications for TDM are the use of over the counter (OTC) drugs and counterfeit drugs from the internet [741, 1093, 1302]. The counterfeit medications may not comply with purity and dos- age standards and therefore increase the risk for adverse drug reactions.

In pharmacovigilance programs, the safety of drug use is supervised under naturalistic conditions $[379,444,445,450,470,648$, $662,946]$. In case of observed adverse events, measurement of drug concentrations in blood is often essential for clarification [569].

\subsection{Recommendations for measuring drug concen- trations in blood}

The usefulness of TDM varies with the clinical situation and the particular drug involved. In case of suspected non-adherence or incomplete adherence (compliance) to medication or intoxications, quantifying drug concentrations in blood is a generally accepted tool for all drugs and groups of patients. However, it is still a matter of debate in many countries whether TDM should be implemented in clinical routine. Based on empirical evidence, four levels of recommendation to use TDM were defined ranging from "strongly recommended" to "potentially useful" as follows:

\section{Definitions}

\section{Level 1: Strongly recommended}

Evidence: Reported therapeutic reference ranges are established. Controlled clinical trials have shown beneficial effects of TDM. Reports on decreased tolerability or intoxications exist. Recommendation: TDM is strongly recommended for dose titration and for special indications. E.g., for lithium or carbamazepine, TDM is a standard of care.

Clinical consequences: At drug concentrations in blood within the reported therapeutic reference range, highest probability of response or remission can be expected. At subtherapeutic drug concentrations in blood, the response rate is similar to placebo under acute treatment and there is a risk of relapse under chronic treatment. At supratherapeutic drug concentrations in blood, there is an increased risk of adverse drug reactions or outright toxicity.

\section{Level 2: Recommended}

Evidence: Reported therapeutic reference ranges were obtained from drug concentrations at therapeutically effective doses and related to clinical effects; there are reports on decreased tolerability or adverse effects at "supratherapeutic" drug concentrations in blood.

Recommendation: TDM is recommended for dose titration and for special indications or problem solving.

Clinical consequences: TDM will increase the probability of response in non-responders. At subtherapeutic drug concentrations, there is a risk of poor response. At supratherapeutic drug concentrations, there is an increased risk of intolerance or intoxication.

\section{Level 3: Useful}

Evidence: Reported therapeutic reference ranges were computed from drug concentrations at approved doses. Drug concentrations related to medication effects either are not yet 
available or are based on retrospective analyses of TDM data, single case reports or non-systematic clinical experience.

Recommendation: TDM is useful for special indications or problem solving.

Clinical consequences: TDM can be used to control whether drug concentrations are in accordance with the dose-related reference range. Clinical improvement may be attained by dose increase in non-responders who display low drug concentrations.

\section{Level 4: Potentially useful}

Evidence: Drug concentrations in blood do not correlate with clinical effects due to unique pharmacology of the drug, e. g., irreversible blockade of an enzyme, or dosing can be easily guided by clinical symptoms, e. g., sleep induction by a hypnotic drug.

Recommendation: TDM is not recommended for dose titration, but may be potentially useful for special indications or problem solving.

Clinical consequences: TDM should be restricted to special indications.

According to our evidence-based evaluation, TDM was graded as "strongly recommended" for 19 of the 154 surveyed neuropsychiatric drugs, "recommended" for 39 , “useful” for 61 , and “potentially useful” for 35 drugs ( Table 4). TDM is strongly recommended for most tricyclic antidepressants. It reduces the risk of toxicity $[168,669,827,934,959,961,964,1304]$. For many tricyclic antidepressants, a concentration - clinical effectiveness relationship (concentration-effect curve) has been shown. For selective serotonin reuptake inhibitors (SSRIs) a weak but significant dose dependence of clinical improvement was reported, whereas tolerability decreased at high doses [564]. Though acceptance of TDM is actually limited in clinical practice $[8,974,1175]$, evidence for its usefulness is growing. For citalopram it has been shown that it is advantageous to use TDM in the early phase of treatment, i. e. one week after start of the medical treatment [896]. Another limitation to introduce TDM for SSRIs is poor methodology when analyzing drug concentrations in blood in relation to clinical effects. Using adequate methodology re-analysis of data on paroxetine concentrations and clinical improvement for which no concentration-response relationship was originally concluded [1175] found a clearcut correlation which was almost identical with the in vivo occupancy of serotonin transporters [329]. Toxicity of SSRIs is low in comparison to most of the pre-SSRI antidepressants $[79,277,526,1178,1297]$. Evidence for a statistically significant relationship between drug concentration and therapeutic outcome is lacking for the tetracyclic antidepressants maprotiline, mianserin and mirtazapine and also for trazodone and reboxetine, as well as for the monoamine oxidase inhibitors moclobemide and tranylcypromine.

TDM is strongly recommended for the typical (first-generation) antipsychotic drugs haloperidol, perphenazine and fluphenazine, and for the atypical (second-generation) antipsychotics amisul- pride, clozapine and olanzapine ( $\triangleright$ Table 4). Overdosing may lead to extrapyramidal symptoms. In the case of clozapine, there is a strong correlation between clozapine concentration in blood and incidence of seizures. TDM-based prevention of overdosing is, for the majority of patients treated with a typical antipsychotic, a matter of the patient's quality of life rather than of safety [237]. TDM of antipsychotics is also useful when medication is switched from the oral to the depot formulation, or vice versa.

Depot formulations of several first and second generation antipsychotics (risperidone, paliperidone, olanzapine, aripiprazole) are often recommended to address non-adherence in patients with schizophrenia. It has been assumed that stable blood levels of depot antipsychotics are associated with superior tolerability and efficacy. However, differences in effectiveness (i. e. relapse prevention) or side-effects of depot and oral antipsychotics have not been clearly evidenced and seem to depend more on the specific compound and dose or drug concentrations in blood [640,641]. Accordingly, steady-state peak-to-trough fluctuations of drug concentrations in blood (see - Fig. 2) are not generally lower in depot formulations (depending on tmax and t1/2) [1078], and not all studies have found a positive correlation of large blood level fluctuations and increased adverse events. For the available depot antipsychotics, pharmacokinetic studies are scarce, and recommended (therapeutic) blood levels of depot and other formulations are almost identical $[28,1113]$.

With regard to the mood stabilizing and/or antimanic drugs lithium, valproic acid and carbamazepine, therapeutic reference ranges and toxic levels are well defined. Therefore, TDM is strongly recommended for these drugs ( $\triangleright$ Table 4). For lithium, TDM has been established as standard of care $[230,281,317,463,707,1076,12$ $83,1307]$. For lithium long-term use, concentrations of $0.5-$ $0.8 \mathrm{nmol} / \mathrm{L}$ in blood are recommended. For an acute treatment with lithium, it may be justified to increase its concentrations up to $1.2 \mathrm{mmol} / \mathrm{L}$.

Compounds that have been shown to be effective as antidementia drugs are donepezil, rivastigmine, galantamine and memantine. TDM is rarely used for the treatment of dementia [468], although there is evidence that it can be useful. For donepezil, it has been shown that the patients' improvement was significantly greater when their concentrations in blood were above $50 \mathrm{ng} / \mathrm{mL}$ as compared to patients that showed lower donepezil concentrations [499, 1013].

Most anxiolytic and hypnotic drugs belong to the pharmacologic class of benzodiazepines. For alprazolam, TDM may be useful to suppress panic attacks [1310]. Most anxiolytic and hypnotic effects are rapid in onset. Treatment is therefore preferentially guided by immediate clinical impression rather than by TDM. Measurements, however, can be informative to identify chronic use of the drugs. In case of lack of therapeutic effects under usual doses, TDM may clarify if non-response is due to drug abuse that has led to tolerance or the result of pharmacokinetic abnormalities. Due to adaptive changes in chronic users, blood concentrations of benzodiazepines poorly correlate with driving performance [1254].

TDM is recommended for the opioid agonists racemic methadone, R-(-)-methadone (levomethadone), buprenorphine and morphine for safety reasons [163]. It must be considered that, similar to benzodiazepines, optimal drug concentrations may vary mark- 
Pre-TDM: Indication for TDM (Table 7)? Availability of laboratory and pharmacological advise?

\section{TDM request \\ Completed request form (Fig. 5): demographic data, diagnosis, medication, \\ clinical situation (improvement, adverse drug reactions etc.)}

\section{Routine monitoring}

Dose titration

Maintenance therapy for relapse prevention

\section{Specific problem}

Insufficient response, suggested non-adherence, adverse drug reaction at therapeutic doses or potential drug-drug interaction (Table 7)

\section{Blood sample collection, storage and shipment}

$\downarrow$

Steady-state at the time of minimal drug concentration (trough level, Cmin)

\section{Laboratory measurement}

Use of validated method (linearity, accuracy, precision, selectivity, sensitivity, specificity)

Internal and external quality controls

\section{Interpretation and communication of results}

Concentration of drug (and metabolite), unit, dose-related and therapeutic reference ranges

(Tables 5 and 6), interpretation (Tables 1 to 6 )

\section{Clinical decision making}

Dose correction, continuation or change of medication

\section{Further supervision of pharmacotherapy}

- Fig. 4 The TDM process to guide neuropsychopharmacotherapy. Routine TDM is primarily applied to drugs with a narrow therapeutic index and a well-defined therapeutic reference range. However, TDM is useful for any neurologic or psychiatric drug when addressing special therapeutic problems.

edly from patient to patient due to different levels of tolerance. On the other hand, opioid dependent patients may ask for higher doses than they can tolerate because of their craving for drugs which can have fatal consequences due to toxic drug concentrations [396, 477, 496, 689]. For "anti-craving" medications such as acamprosate or naltrexone or for the use of alcohol-aversive disulfiram to treat alcohol use disorders or naltrexone in case of opioid addiction for abstinence treatment, TDM is recommended to enhance the moderate efficacy [163]. TDM of drugs to treat substance related disorders should consider preferentially expected drug concentrations ( $\vee$ Table 5 ) to clarify adherence problems, tolerance to medication or pharmacokinetic abnormalities.

For anticonvulsant drugs, TDM is well established, not only for the old drugs, which are relatively toxic [912], but also for new ones [562, 681].

For antiparkinson drugs, TDM has not been established so far. For dopamine agonists, data on reference ranges are scarce. For Ldopa, a moderate correlation between drug concentrations in blood and short-term clinical response is considered [867]. Never- theless, the pharmacokinetic properties of these neurologic drugs have been included in the present guidelines ( $>$ Tables $1-6$ ), because antiparkinson drugs exhibit concentration dependent sedative properties. TDM may avoid overdosing.

\section{Practical Aspects of TDM in Psychiatry and Neurology}

\subsection{TDM request for quantification of drug concen- trations in blood}

Essential for an effective TDM service is the availability of appropriate analytical methods that produce results within a reasonable time, i. e., within $48 \mathrm{~h}$ from the arrival of the blood sample in the laboratory to send the results including advice from someone who understands pharmacokinetics and therapeutics [314]. As shown in $>$ Fig. 4, the TDM process starts with the request and ends with the final decision how to adjust a patient's therapeutic regimen by the health care professional. 


LABORATORY
Address
Phone
Fax

REQUESTING HOSPITAL / DOCTOR

Address

Phone in case of alert

Fax

\begin{tabular}{|l|l|l|l|}
\hline PATIENT DETAILS & Name or code & $\square$ Inpatient $\square$ Outpatient & Date and time of blood withdrawal \\
\hline Date of birth & Sex & Diagnosis / Symptom(s) \\
\hline$\square$ HIV-patient & Weight $(\mathrm{kg})$ & $\begin{array}{l}\text { Smoker } \square \text { No } \square \text { Moderate (<10 cig/day) } \\
\text { Genotype/phenotype to be considered (e.g. CYP2D6, 2C19, 1A2): }\end{array}$ \\
\hline
\end{tabular}

\begin{tabular}{|c|c|c|c|c|}
\hline $\begin{array}{l}\text { REASON FOR REQUEST } \\
\text { (tick more than one if applicable) } \\
\square \text { Control of adherence }\end{array}$ & \multicolumn{2}{|c|}{$\begin{array}{l}\square \text { Dose adaptation } \\
\square \text { Insufficient improvement } \\
\square \text { Adverse drug reaction (to be specified) ....................... }\end{array}$} & \multicolumn{2}{|c|}{$\begin{array}{l}\square \text { Drug-drug interaction } \\
\square \text { Control under maintenance therapy } \\
\square \text { Other reason (to be specified) .......... }\end{array}$} \\
\hline \multirow{2}{*}{$\begin{array}{l}\text { SEVERITY OF ILLNESS } \\
\text { (CGI-S) } \\
\text { How mentally ill is the patient } \\
\text { at this time? } \\
\square \text { Not at all ill (1) } \\
\square \text { Borderline mentally ill (2) } \\
\square \text { Mildly ill (3) } \\
\square \text { Moderately ill (4) } \\
\square \text { Markedly ill (5) } \\
\square \text { Severely ill (6) } \\
\square \text { Extremely ill (7) }\end{array}$} & \multirow{2}{*}{$\begin{array}{l}\text { IMPROVEMENT } \\
\text { (CGI-I) } \\
\text { Change compared to } \\
\text { condition at admission? } \\
\square \text { Very much improved (1) } \\
\square \text { Much improved (2) } \\
\square \text { Minimally improved (3) } \\
\square \text { No change (4) } \\
\square \text { Minimally worse (5) } \\
\square \text { Much worse (6) } \\
\square \text { Very much worse (7) }\end{array}$} & \multicolumn{3}{|c|}{ ADVERSE DRUG REACTION (UKU) } \\
\hline & & $\begin{array}{l}\square \text { Concentration difficulties } \\
\square \text { Tension/inner unrest } \\
\square \text { Dystonia } \quad \square \text { Rigidity } \\
\square \text { Akathisia } \quad \square \text { Epileptic } \\
\square \text { Accomodation disturban } \\
\square \text { Nausea/Vomiting } \square \mathrm{D} \\
\square \text { Polyuria/Polydypsia } \square \text { In } \\
\square \text { Sexual dysfunction } \quad \square \text { O }\end{array}$ & $\begin{array}{l}\square \text { Asthenia } \\
\square \text { Sleep disturbances } \\
\square \text { Akinesia } \\
\text { izures } \square \text { Paresthesias } \\
\text { arrhoea } \quad \square \text { Constipation } \\
\text { reased sweating } \square \text { G } \\
\text { her (to be specified) } \\
\text { sal relationship: } \square \text { imp }\end{array}$ & $\begin{array}{l}\square \text { Sleepiness/Sedation } \\
\square \text { Emotional indifference } \\
\square \text { Hypokinesia } \square \text { Tremor } \\
\square \text { Headache } \\
\text { ation } \square \text { Dry mouth } \\
\square \text { Micturation disturbance } \\
\text { actorrhoea } \quad \square \text { Weight gain } \\
\text { bbable } \square \text { possible } \square \text { probable }\end{array}$ \\
\hline
\end{tabular}

\begin{tabular}{|l|l|l|l|l|}
\hline Drug(s) to be assayed & Formulation & Daily dose / dosing schedule & Date started & Time of last dose \\
\hline & & & & \\
\hline & & & & \\
\hline
\end{tabular}

Other medications (include herbals, over the counter drugs etc.)

TDM request: Blood should be withdrawn under steady-state conditions, preferably in the morning BEFORE taking the morning dose.

Return the completed form, together with a minimum of $1 \mathrm{~mL}$ serum or plasma.
Date of sample receipt:

Signature:

- Fig. 5 Example of a request form recommended for therapeutic drug monitoring of neuropsychiatric drugs.

As mentioned above, TDM should only be requested when there is evidence that the result will provide an answer to a specific question. Typical indications are listed in $>$ Table 7. A single measurement is often insufficient for problem solving. For example, a series of measurements may be required at appropriate intervals to clarify if a low drug concentration in blood is either due to poor adherence, reduced bioavailability or abnormally rapid elimination.

TDM requests must include a completed request form ( $\triangleright$ Fig. 5), which is essential for effective drug concentration measurements and an adequate interpretation of the results $[923,1159]$. The form should contain the patient's name or code, demographic data, diagnosis, medication, reason for the request, the commercial and the generic name of the drug and its dose, the galenic formulation, the time of the last change of the dose, time of drug intake, time of blood withdrawal. A brief comment on the clinical situation should be given for interpretation of the results. As indicated in - Fig. 5, we recommend to use symptom rating scales, e. g., the clinical global impression (CGI) [467], to measure the severity of illness (CGI-S) and document any therapeutic improvement or worsening (CGI-I). The summary form of the UKU scale is useful to evaluate the occurrence and severity of adverse drug reactions [717]. However, documented feedback to questionnaires indicates that clinicians often do NOT want to write that much information on the form. Moreover, the filled-in information is often not accurate. On the other hand, the completed request form is a case document for the physician to review the pharmacotherapy and a suitable training device to learn TDM. As an alternative, feedback by phone may be offered for interested physicians. Adding the website address of the lab will facilitate the download of request forms and other documents by the client.

When interpretation of the results is requested from the laboratory, it is necessary to fill out the request forms adequately. Computerized ordering of TDM has advantages. It is inexpensive and it guides the ordering physician to give the relevant information required for interpretation in a comfortable way.

\subsection{Specimen collection}

\subsubsection{Blood sample collection}

Generally, TDM is carried out in plasma or serum samples. There is no consensus whether plasma or serum should be preferred. Definite experimental data that unequivocally demonstrate differences in the drug concentrations using either plasma or serum are still lacking. The few available comparisons indicate that values obtained from serum or plasma can be used interchangeably [513]. For most laboratories the collection tubes should not contain EDTA, 
citrate, heparin or other additives. An amount of one $\mathrm{mL}$ plasma or serum is sufficient for most laboratories. Concentrations of neuropsychiatric drugs reported in this guideline refer to the total drug fraction in accordance with the literature. There is no experimental evidence for the hypothesis, that the assay of unbound ("free") drug concentrations in blood would be advantageous. Moreover, the assay of the free fraction represents an analytical challenge [87]. For imipramine, it has been shown that the drug is rapidly and almost totally cleared by the brain through a single passage in the microvasculature [994]. The extraction was not significantly affected in the presence of albumin, lipoproteins or erythrocytes. For nortriptyline, statistical relationships between free levels of drug and clinical response were found to be insignificant [929]. Therefore, at least for psychiatric drugs, it seems likely that the clinical response depends on the total drug fraction. With the exception of saliva, analysis of neuropsychiatric drugs in other materials such as urine, spinal fluid, tears, hairs or maternal milk have not been introduced for TDM purposes, and no validated data are available which deal with therapeutic concentrations.

Blood collection via dried blood spots can be an alternative to the common venous blood withdrawal. The minimally invasive sampling, low blood volume requirements, easy transport and storage and good analyte stability are key advantages of this sampling method. The high sensitivity of modern analytical techniques such as liquid chromatography-tandem mass spectrometry (LC-MS/MS) or ultra-high performance liquid chromatography-tandem mass spectrometry (UPLC-MS/MS) allows the use of dried blood samples for TDM $[810,913,916,1303]$. Thereby, several points need to be taken into consideration: dried blood sample concentrations must be corrected for plasma/serum concentrations, the influence of haematocrit, the influence of the collected blood volume and various types of filter paper. Volume defining dried blood sampling techniques and automated techniques such as online desorption, paper spray analysis and fully automated extraction of dried blood samples are already available. However, they require further clinical validation in order to make dried blood spots sampling a suitable and cost-effective alternative to whole blood sampling in a clinical routine laboratory providing TDM [1303].

With regard to the timing of blood collection, it must be considered that TDM guided neuropsychopharmacotherapy mostly relies on minimal drug concentrations ( $\mathrm{Cmin}$ ) at steady-state. Steady-state is reached under constant doses after at least 4 to 6 elimination half-lives (see $>$ Table 4 ) and $C$ min at the end of the longest dosing interval. For practicability, most blood samples taken for determination of $\mathrm{Cmin}$ are withdrawn in the morning before the first dose of the day, which is mostly the time of minimal drug concentrations (tmin). A frequent problem, however, is blood sampling at different time points throughout the dosing interval. This leads to concentrations that may be misinterpreted when in reality true trough levels are lower or higher. For antibiotics, it has been reported that up to $55 \%$ of inappropriate levels were due to improper timing of sample collections [1205].

For antiparkinson drugs and drugs like methylphenidate for the treatment of attention-deficit hyperactivity disorder blood is withdrawn at tmax, the time of maximal drug concentrations (Cmax). Most of these drugs have a short elimination half-life and clinical effects correlate with Cmax.

\section{Blood sampling under treatment with depot or extended release formulations}

In patients treated with a depot formulation of an antipsychotic drug, blood should be sampled immediately before the next injection. The drug concentration in blood depends on the release from the depot and the elimination. TDM may of course be carried out at any time if unexpected adverse drug reactions are observed. It is not necessary to measure trough levels, but the dosing schedule should be reported for interpretation.

Long acting formulations of antipsychotic drugs such as haloperidol decanoate or risperidone and aripiprazole are characterised by a slow absorption after intramuscular administration. Maximum concentrations in blood of first generation depot antipsychotics are reached 1-14 days after injection, and the apparent elimination half-life of the depot is $2-3$ weeks [1179]. Paliperidone palmitate exhibits similar properties [1113] with an apparent elimination half-life ranging between 25 and 49 days [976]. For risperidone microspheres, the mean time to peak concentrations is 4 weeks and its apparent elimination half-life 4-6 days [1179]. Long-acting olanzapine pamoate [714] slowly releases olanzapine from the injection site into the muscle tissue. However, it dissolves rapidly when it is in contact with blood or plasma. The latter results in high concentrations in blood and may lead to marked sedation and delirium, the so-called post-injection syndrome [714, 1179]. Due to the low solubility, the absorption of aripiprazole depot (once monthly) is slow and prolonged with an apparent average absorption half-life of 4 weeks. Maximal drug concentrations are reached in blood 5-7 days after injection; the mean apparent terminal elimination half-life after 400 or $300 \mathrm{mg}$ aripiprazole monthly is 47 and 30 days, respectively $[365,751]$.

For oral drugs delivered in extended release formulations like venlafaxine, methylphenidate, paliperidone [110] or quetiapine [356], special attention has to be given to the time of drug intake for correct interpretation (see - Table 4). In these formulations, the time of maximal drug concentration in blood is delayed too, whereas the terminal elimination half-life of the drugs is essentially unchanged.

\subsubsection{Oral fluid for TDM}

Oral fluid offers the advantage of non-invasive collection $[30,39,613]$. It has been applied to optimize the treatment with a few antiepileptic drugs [911] for confirmatory purposes [683] and qualitative interpretations of results [914]. It has been long assumed that drug concentrations in oral fluid reflect the free fraction (i. e., non-protein-bound) that circulates in blood and which for most psychopharmacological drugs is only $10 \%$ or less of their total concentration. Detection problems were therefore a major problem in the past when using saliva instead of blood plasma or serum. Improved methods are now available to analyse saliva with sufficient precision and accuracy $[913,914]$. Using such techniques it was found that the ratio of concentrations in blood to saliva differed a lot and did not fully support the assumption that saliva contains the free fraction of the drug in blood. Comparisons of drug concentrations in blood and oral fluid revealed that oral fluid will actually not replace blood as matrix for TDM [914]. There was an apparent positive correlation between the concentration of monohydroxyoxcarbazepine (MHD, the major metabolite of oxcarbazepine) in blood plasma and saliva [706]. For carbamazepine, pheny- 
toin and phenobarbital the correlation was poor but still significant [316]. For valproic acid, however, the correlation was not significant [315]. It was reported that saliva cannot replace blood for monitoring of methadone [1084].

For amitriptyline and nortriptyline, no significant relationship was found between concentrations in saliva and plasma [87]. Many neuropsychiatric drugs are bases, with a pKa value $>9$. The distribution of drugs between blood and saliva depends on the $\mathrm{pH}$. The $\mathrm{pH}$ of saliva increases when the secretion is stimulated. For methylphenidate, an inverse correlation was found for the ratio of drug concentration in oral fluid to serum and $\mathrm{pH}$ value of oral fluid samples [1133]. Standardization and optimizing of sampling [682] is needed. In any case, more data are required for measurement of drug concentrations in saliva as a matrix.

\subsection{Storage and shipment of blood samples}

With few exceptions, serum or plasma samples can be stored in the dark at $4^{\circ} \mathrm{C}$ for at least $24 \mathrm{~h}$, and most drug samples can be sent without freezing [506]. Exceptions are light and/or oxygen sensitive substances like bupropion or methylphenidate. For their determination, samples must be stabilized by freezing or extraction immediately after blood withdrawal and centrifugation (see - Table 4). For determination of olanzapine, serum or plasma samples must be stored frozen $\left(-20^{\circ} \mathrm{C}\right)$ if not analysed within $72 \mathrm{~h}$ [506]. When samples must be stored and sent frozen, it is required to prepare serum or plasma before freezing, since it is not possible to prepare serum or plasma from frozen blood. The laboratory should give instructions on its web site or the request form how to collect (plasma volume, labelling of the samples), store and mail the sample.

\subsection{Laboratory measurements}

Selective and sensitive analytical methods for the quantitative evaluations of the analytes, i.e., the drugs and their metabolites, are essential for the successful application of TDM. Methods have to be validated [185, 715]. The validation includes all procedures demonstrating that a particular method used for quantitative measurement of analytes in a given biological matrix is reliable and reproducible for its intended use. The fundamental parameters for this validation comprise (1) accuracy, (2) precision, (3) selectivity, (4) sensitivity, (5) reproducibility and (6) stability. Validation involves documenting that the performance characteristics of the method are suitable and reliable for the intended analytical procedure. The acceptability of analytical data corresponds directly to the criteria used to validate the method [185, 370 and see: http://www.ema. europa.eu/docs/en_GB/document_library/Scientific_guideline/2011/08/WC500109686.pdf].

For neuropsychiatric compounds, chromatographic techniques (preferentially high-performance liquid chromatography, HPLC), in combination with suitable detection methods, are preferred [318]. They are sufficiently precise, accurate and robust and can be adapted to the analysis of almost every neurologic or psychiatric drug. A disadvantage is the need for sample preparation before chromatographic separation and hence a limited sample throughput. Throughput can be enhanced by automated sample preparation prior to HPLC. Some laboratories have introduced HPLC with column switching which allows direct injection of plasma or serum into the HPLC system. Such procedures are available for a number of antidepres- sant $[446,486,487,490,491,1274,1288]$ and antipsychotic drugs [638, 639, 1026-1028, 1287, 1289]. Another high-throughput chromatographic method is liquid chromatography coupled with mass spectroscopy (LC-MS), especially tandem MS (LC-MS/MS) [1032]. LC-MS/MS is most sensitive and selective. Additionally, this technique can be applied with minimal sample preparation such as protein precipitation and dilution. Many compounds can be analysed simultaneously. An excellent example is the LC-MS/MS method described by Kirchherr and Kühn-Velten [635] that was validated for over 50 psychoactive drugs. Major disadvantages of LC-MS/MS methods are high equipment costs and the need for well trained personnel. Moreover, quantification can be jeopardized due to matrix effects and ion suppression. These effects can be minimized by good chromatographic separation of matrix and the analyte of interest and the use of stable isotopically labelled standards for internal calibration, preferentially deuterated analogues [1047]. During the last years, therefore, LC-MS/MS methods are used with increasing frequency $[37,914,915,917]$. Their big advantage is flexibility. Their disadvantage of high costs was gradually reduced to acceptable prices. LC-MS/ MS is nowadays the preferred analytic method for TDM of neuropsychiatric drugs in many specialized laboratories. HPLC with UV- or fluorescence detection is, however, still the established method of choice in many laboratories of low to medium throughput due to its cost effectiveness and robustness.

In case of suspected intoxications, TDM methods should allow drug analysis within 1-2 h [364]. For this purpose, automated methods are advantageous. The use of LC-MS/MS in this special application is advantageous due to the high selectivity of mass spectrometry for identification.

The assay of enantiomers of chiral compounds requires either stereoselective derivatisation of the drugs prior to their quantification, or their separation by chiral chromatographic columns. For detection tandem mass spectrometry is the method of choice. As an example, the TDM of the enantiomers of methadone using a classical detection method such as fluorescence or ultraviolet light absorption is often jeopardized by co-medication or by co-consumption of drugs of abuse. These problems may be circumvented by use of a mass detector, preferably a tandem mass spectrometer.

Within the therapeutic reference range, intraday- and interday precision should not exceed $15 \%$ (coefficient of variation) and accuracy should not deviate more than $15 \%$ from the nominal value [185, 370].

To ensure quality and reliability of drug assays, internal and external quality control procedures are mandatory. Samples must contain suitable internal standards, and each series of samples must include internal control samples. If standards are not available commercially, they should be prepared by personnel other than that performing the assays and by separate weighing of reference material. Commercial quality control samples are increasingly available spanning a wide range of psychoactive drugs today. Reporting of results requires that the results of the quality controls are within the expected ranges. If quality controls are outside the expected range, the reason underlying the outlier needs to be clarified and documented.

The laboratory has to participate in an external quality assessment scheme, although this is not a legal requirement in all countries. For neuropsychiatric drugs, the first external quality program 
was introduced by Cardiff Bioanalytical Services Ltd in 1972 [1306]. The service was taken over by other providers of external quality control schemes like LGC (www.lgcstandards.com) or Instand e. V. (www.instand-ev.de). Moreover, reference materials are also available from the Task Force of Clinical Toxicology of the Society of Toxicological and Forensic Chemistry (www.gtfch.org).

\subsection{Computing of trough steady-state concentrations}

When comparing drug concentrations measured by TDM and expected steady-state $\mathrm{Cmin}$, it is assumed that blood was withdrawn at the time of minimal drug concentrations (tmin). To measure steady-state $\mathrm{Cmin}$, blood should be collected after at least 4 drug elimination half-lives after the start of medication or a change in dosage and during the terminal ß-elimination phase. For most psychiatric and neurologic drugs, elimination half-lives vary between 12 and $36 \mathrm{~h}$ ( $>$ Table 4). Notable exceptions are quetiapine, venlafaxine or trazodone which display elimination half-lives around $6 \mathrm{~h}$. Fluoxetine, donepezil and aripiprazole have longer elimination halflives. In clinical practice, the appropriate sampling time for most neurologic and psychiatric drugs is one week after stable daily dosing and immediately before ingestion of the morning dose, which usually is $12-16 \mathrm{~h}$ (or $24 \mathrm{~h}$ when the drug is given once daily in the morning) after the last medication. If, for logistic reasons, blood can only be collected late in the morning, the patient should not be medicated before blood withdrawal. In an outpatient setting, it is important to indicate exactly the time of administration of the last dose to be able to calculate expected trough levels. This can be done by the following Eq. (5).

$\mathrm{Cmin}=\mathrm{Ct} \times \mathrm{e}^{-\mathrm{ke} \cdot(\mathrm{tmin}-\mathrm{t})}$

where $\mathrm{Ct}$ is the drug concentration measured at time $\mathrm{t}$, tmin the time at $\mathrm{Cmin}$ and ke the elimination rate constant $\left(\mathrm{ke}=\ln 2 / \mathrm{t}_{1 / 2}\right)$.

As an example it is given that amisulpride, which has an average elimination half-life of $16 \mathrm{~h}$ (see $>$ Table 5 , ke $=0.0433 \mathrm{~h}^{-1}$ ), was applied daily as single dose per day (at 08:00 h). On the day of blood withdrawal, the patient did not take the medication, since he was instructed to take it after blood withdrawal for TDM. Because of organizational reasons, blood was finally withdrawn at 11:00 h in the morning. When the measured drug concentration (Ct) was $351 \mathrm{ng} /$ $\mathrm{mL}, \mathrm{Cmin}$ at time $24 \mathrm{~h}$ ( = tmin) should amount to

$351 \cdot \mathrm{e}^{-0.0433(24-27)}=399 \mathrm{ng} / \mathrm{mL}$

Eq. (5) can also be used for estimation of Cmin when blood was withdrawn in the postabsorptive phase before tmin was reached.

Given e. g., that lithium, which has an elimination half-life of $24 \mathrm{~h}$ (see - Table 5), was applied as single dose per day in the evening at 20:00 h, blood was withdrawn at 08:00 $\mathrm{h}$ in the morning $(\mathrm{t}=12 \mathrm{~h})$ and the measured drug concentration $(\mathrm{Ct})$ was $1.0 \mathrm{mmol} / \mathrm{l}$, then $\mathrm{Cmin}$ at time $24 \mathrm{~h}$ ( = tmin) should amount to

$$
1.0 \cdot \mathrm{e}^{-\mathrm{ke}(24-12)}=0.71 \mathrm{mmol} / \mathrm{l}
$$

\subsection{Interpretation and communication of results and recommendations}

The concentration of the neuropsychiatric drug as well as that of its active metabolites contributing to the therapeutic action should be reported together with reference ranges ( $\triangleright$ Table 4 ), either in mass or molar units. We recommend the use of mass units instead of molar units to relate concentration to dose. Laboratories vary in the presentation of their results. The clinician should take note of the units (i. e., $\mathrm{ng} / \mathrm{mL}, \mu \mathrm{g} / \mathrm{L}, \mu \mathrm{mol} / \mathrm{L}$, or $\mathrm{nmol} / \mathrm{L}$ ) in which the results of the analysis are expressed. This is especially recommended for comparisons of values obtained from different laboratories or with those in the literature. To transform molar units into mass units and vice versa conversion factors are given in $>$ Table 4.

When drug concentrations are below the lower limit of quantification, which refers to the lowest concentration of the standard curve that can be measured with at least $80-120 \%$ accuracy and $20 \%$ precision, this limit should be indicated [370].

The results should be available for decision making within a clinically meaningful time. A $24 \mathrm{~h}$ TDM service is desirable, however, a $48 \mathrm{~h}$ turnaround time is sufficient in most cases. In case of suspected intoxications, a few hours service is necessary [364]. To assist rapid intervention in patients at risk for toxicity or loss of tolerability, prompt information of the treating physician (i.e., a phone call) is required when the laboratory measures drug concentrations above the laboratory alert level ( $\triangleright$ Table 4).

We highly recommend that interpretation and pharmacologic advice are provided with every assessment of a drug concentration. Expert interpretation and the adequate use of the information are essential to ensure the full clinical benefit of TDM report [82, 314, $469,471,519,979,1159]$. Reporting of results with inclusion of dose recommendations and other comments must be guided by the best available evidence. Expert knowledge may be necessary to calculate dose corrections or to analyse drug-drug interactions. It is advantageous for the clinician to choose a laboratory that offers this service. Otherwise, the treating physician, a clinical pharmacologist or a trained expert of the clinic has to interpret the results. Access to specialist advice is also necessary if TDM results suggest that genotyping may be advisable.

It may even be legally required to include collaboration with a clinical pharmacologist. In Switzerland, a psychiatrist may prescribe CYP genotyping, but it will only be reimbursed by insurances, when the test is prescribed by a physician specialized in clinical pharmacology.

Diagnosis and drug dose are important for interpretation, since they permit a judgment on whether a result is plausible or not. Moreover, it must be checked whether blood samples were collected under recommended conditions, especially when the drug concentration in blood is unexpectedly high in an outpatient. When the drug was taken only a few hours before blood sampling, the drug concentration can be several-fold higher than the trough level ( $\triangleright$ Fig. 2). The trough steady-state concentration can be easily calculated by Eq. (5) when blood was withdrawn in the postabsorptive phase.

For interpretation of the results, it must be checked whether the concentration of the drug in blood is within the therapeutic reference range ( $\triangleright$ Table 4 ) and fits to the dosage ( $\triangleright$ Table 5). When a drug concentration is outside the therapeutic reference range, it is wise to take into account the level of recommendation underlying the therapeutic reference range of the particular drug ( $\triangleright$ Table 4). Any drug concentration outside its dose-related reference range ( $\triangleright$ Table 5) should alert the TDM laboratory to actively look for drug-drug-interactions or gene polymorphisms that give rise to poor or ultrarapid metabolism, altered function of the excretion organs liver and kidneys, age and/or disease-related changes in the 
patient's pharmacokinetics, adherence problems, a non-steadystate and even signal interference from other medications that the patient may not have declared to the prescribing physician (e.g., St. John's wort). It should also be considered whether the daily drug dose was given as a single or a multiple dose regimen.

Often it is necessary to deal with metabolic pathways, enzymes involved and substrate and inhibitor properties of all drugs taken by the patient for interpretation of the results. Supportive information is therefore given in the present updated guidelines showing literature based substrate ( $\vee$ Table 1 ) and inhibitor or inducer properties of drugs ( $\vee$ Tables 2,3 ) as causes of possible drug-drug interactions.

For the treatment of pain, relatively low concentrations of tricyclic antidepressants may be sufficient. They may be within the doserelated reference range ( $\vee$ Table 5 ) but outside the therapeutic reference range of $\triangleright$ Table 4 , which was established for the indication of depression.

A laboratory may recommend that an additional sample should be taken after a certain period, because in cases with unusually low or high drug concentrations, repeated measurements may help to decide whether the patient's adherence is inconstant (irregular intake of the drug) or whether the patient is an ultrarapid or poor metabolizer.

Recommendations must be given with the clinical presentation in mind as explained for the cases below. Dosage changes constitute the most frequent advice.

\subsubsection{How to use the TDM guidelines for interpretation of} results - cases

To demonstrate how to use information of the consensus guidelines for interpretation of laboratory results, three representative cases are shown below.

\begin{tabular}{|c|c|}
\hline \multicolumn{2}{|l|}{ Case 1} \\
\hline Patient: & $\begin{array}{l}51 \text { years/male/inpatient/ } \\
\text { smoker (> } 10 \text { cig./day) }\end{array}$ \\
\hline Diagnosis: & Paranoid schizophrenia \\
\hline Reason for request: & Uncertain adherence \\
\hline Severity of illness: & Severely ill (CGI-S score 6) \\
\hline Improvement: & No change (CGI-I score 4) \\
\hline Adverse drug reactions: & Not reported \\
\hline Drugs to be assayed/dose: & Clozapine/250 mg/d \\
\hline Start of medication: & 5 weeks before \\
\hline Last change of dose: & 2 weeks before \\
\hline Last drug intake: & $12 \mathrm{~h}$ before \\
\hline Co-medication: & $\begin{array}{l}\text { Acetylsalicylic acid, } \\
\text { simvastatin, sertraline }\end{array}$ \\
\hline \multicolumn{2}{|l|}{ Laboratory results } \\
\hline - Clozapine: & $\begin{array}{l}224 \mathrm{ng} / \mathrm{mL} \text { (Therapeutic } \\
\text { reference range } 350 \text { - } \\
600 \mathrm{ng} / \mathrm{mL} \text {, see }>\text { Table } 4 \text { ) }\end{array}$ \\
\hline - N-Desmethylclozapine: & $175 \mathrm{ng} / \mathrm{mL}$ \\
\hline
\end{tabular}

\section{Interpretation}

TDM was indicated in accordance with the consensus guidelines ( $\triangleright$ Table 7). Under a therapeutic dose of $250 \mathrm{mg}$, the patient did not improve according to the assessment by the Clinical Global Impressions (CGI-I) scale (see - Fig. 5). TDM had to clarify whether the patient was non-adherent or whether the dose should be increased to improve therapeutic efficacy.

Determination of clozapine revealed a concentration of $224 \mathrm{ng} / \mathrm{mL}$, which is below the therapeutic reference range of 350 to $600 \mathrm{ng} / \mathrm{mL}$ (see $>$ Table 4) but within the dose-related reference range for clozapine and its metabolite ( $\triangleright$ Table 5). At a dose of $250 \mathrm{mg} /$ day the expected dose-related reference ranges (for calculation see DRC factors low and high in Table 5) are $250 \times 0.43=108$ to $250 \times 1.59=398 \mathrm{ng} / \mathrm{mL}$ for clozapine, and $250 \times 0.50=125$ to $250 \times 1.25=313 \mathrm{ng} / \mathrm{mL}$ for $\mathrm{N}$-desmethylclozapine. The ratio of concentrations for $\mathrm{N}$-desmethylclozapine to clozapine was 0.78 and thus as expected for the metabolite to parent compound ratio (MPR) of 0.45 to 0.78 (see $>$ Table 6). The patient was a smoker. $>$ Table 2 does not indicate an inhibitor within the list of comedications, but $>$ Table $\mathbf{3}$ indicates that smoking induces CYP1A2 which is involved in the metabolism of clozapine ( $\triangleright$ Table 1 ).

\section{Recommendation}

Dose increase is recommended to improve efficacy. From the concentration to dose ratio of $0.9 \mathrm{ng} / \mathrm{mL} / \mathrm{mg}$ it can be assumed that $400 \mathrm{mg} /$ day are required to attain therapeutically recommended concentrations (350-600 ng/mL).

\section{Case 2}

Patient:

70 years/female/inpatient/ smoker (> 10 cig./day)

Diagnosis: Major depressive episode

Reason for request: Adverse drug reaction and clinical improvement

Severity of illness: Moderately ill (CGI-S score 4) Improvement: Much improved (CGI-Iscore 2) Adverse drug reactions: Gastrointestinal disturbance Drugs to be assayed/dose: Venlafaxine XR/225 mg/d Start of medication: 3 weeks before Last change of dose: 1 week before Last drug intake: $24 \mathrm{~h}$ before Co-medication: Levomepromazine

\section{Laboratory results}

- Venlafaxine: $168 \mathrm{ng} / \mathrm{mL}$

- O-Desmethylvenlafaxine:

- Active moiety:

$$
251 \mathrm{ng} / \mathrm{mL}
$$
$419 \mathrm{ng} / \mathrm{mL}$ (Therapeutic reference range $100-$ $400 \mathrm{ng} / \mathrm{mL}$, see $>$ Table 4)

- N-Desmethylvenlafaxine: $143 \mathrm{ng} / \mathrm{mL}$

\section{Interpretation}

TDM was indicated in accordance with the consensus guidelines. Under a therapeutic dose of $225 \mathrm{mg}$, the 70 years old 
patient had adverse drug reactions but was much improved according to the assessment by the Clinical Global Impressions (CGI-I) scale (see - Fig. 5). TDM had to clarify whether adverse drug reactions were associated with high concentrations of venlafaxine active moiety and whether the dose could be lowered without risking loss of therapeutic efficacy.

Determination of drug and metabolite concentrations revealed an active moiety concentration of venlafaxine plus $\mathrm{O}$ desmethylvenlafaxine of $419 \mathrm{ng} / \mathrm{mL}$, which is slightly above the therapeutic reference range of 100 to $400 \mathrm{ng} / \mathrm{mL}$ (see - Table 4) and above the dose-related reference range. At a dose of $225 \mathrm{mg} /$ day the expected dose-related reference ranges (for calculation see DRC factors in > Table 5) are $225 \times 0.12=27$ to $225 \times 0.36=81 \mathrm{ng} / \mathrm{mL}$ for venlafaxine, $225 \times 0.78=176$ to $225 \times 1.30=293 \mathrm{ng} / \mathrm{mL}$ for O-desmethylvenlafaxine. The expected active moiety concentration should amount to $203-376 \mathrm{ng} / \mathrm{mL}$. $>$ Table 1 indicates that venlafaxine is a substrate of CYP2D6 and CYP2C19. The ratio of concentrations for O-desmethylvenlafaxine to venlafaxine was 1.49 and thus below the expected metabolite to parent compound ratio (MPR) of 2.7 to 7.7 (see - Table 6). This points to a PM phenotype of CYP2D6. The ratio of concentrations for $\mathrm{N}$-desmethylvenlafaxine to venlafaxine was 0.85 , which is consistent with a normal CYP2C19 phenotype (see > Table 6). Co-medication was levomepromazine, and the patient was a smoker. - Table 2 indicates that levomepromazine is an inhibitor of CYP2D6, which catalyzes the formation of O-desmethylvenlafaxine and $\triangleright$ Table 3 shows that smoking induces CYP1A2 which is not involved in the metabolism of venlafaxine ( $\triangleright$ Table 1). It thus seemed likely that adverse effects were related to the high drug concentrations possibly due to inhibition of CYP2D6 by levomepromazine. The PM phenotype of CYP2D6 is further confirmed by the higher than expected concentration of $\mathrm{N}$-desmethylvenlafaxine of $143 \mathrm{ng} / \mathrm{mL}$ (expected concentration 34 to $74 \mathrm{ng} / \mathrm{mL}$ ). Since levomepromazine is a substrate of CYP2D6, its concentrations may also be high, especially in a PM genotype, and then contribute to the adverse effects.

\section{Recommendation}

Reported adverse drug reactions can be explained by high concentrations of venlafaxine and $\mathrm{O}$-desmethylvenlafaxine most probably due to a drug-drug interaction and old age. The patient may be a PM phenotype of CYP2D6 because of inhibition by levomepromazine. Dose reduction can be helpful and possibly improve tolerability without risking loss of efficacy. Alternatively, levomepromazine may be replaced by a non CYP inhibiting drug, e. g., pipamperone, since reported gastrointestinal disturbances could also be due to levomepromazine.

\section{Case 3}

Patient:

51 years/male/inpatient/

Diagnosis:

Reason for request: smoker (<10 cig./day)

Bipolar disorder, currently manic

Severity of illness: Improvement: Adverse drug reactions: Poor clinical improvement/ uncertain adherence Markedly ill (CGI-S score 5) No change (CGI-I score 4)

Drugs to be assayed/dose: Valproic acid $/ 900 \mathrm{mg} / \mathrm{d}$

Olanzapine $/ 10 \mathrm{mg} / \mathrm{d}$

Start of medication: > 6 weeks before Last change of doses: 2 weeks before Last drug intake: Co-medication: $12 \mathrm{~h}$ before None

\section{Laboratory results}

- Valproic acid:

$37 \mu \mathrm{g} / \mathrm{mL}$ (Therapeutic range $50-100 \mu \mathrm{g} / \mathrm{mL}$, see - Table 4)

- Olanzapine: $7 \mathrm{ng} / \mathrm{mL}$ (The therapeutic reference range for bipolar disorders is unclear. Considering the dose of $10 \mathrm{mg}$ which is recommended for combination therapies, 8 to $23 \mathrm{ng} / \mathrm{mL}$ may be suggested as an orienting therapeutic range)

- N-Desmethylolanzapine: $2 \mathrm{ng} / \mathrm{mL}$

\section{Interpretation}

TDM was indicated in accordance with the consensus guidelines. According to the CGI-I score of 4 , the patient had not improved (see - Fig. 5). TDM could clarify if the patient has taken his medication as prescribed and if dose escalation could be helpful.

Determination of valproic acid (valproate) revealed a concentration of $37 \mu \mathrm{g} / \mathrm{mL}$ which is below the therapeutic reference range ( $\vee$ Table 4 ) and also lower than the expected doserelated concentration. Calculation of the dose-related reference range (see DRC factors in > Table 5) leads to 55,980 to $121,320 \mathrm{ng} / \mathrm{mL}$ (i. e. $56-121 \mu \mathrm{g} / \mathrm{mL}$ ) for a dose of $900 \mathrm{mg}$ valproic acid. For olanzapine and its metabolite the concentrations were $7 \mathrm{ng} / \mathrm{mL}$ and $2 \mathrm{ng} / \mathrm{mL}$, respectively. These concentrations cannot be related to therapeutic effects, since a therapeutic reference range has not been established for the indication bipolar disorder. At a dose of $10 \mathrm{mg} /$ day, however, the expected concentration can be calculated (see $>$ Table 5 ). They should amount to 12 to $25 \mathrm{ng} / \mathrm{mL}$ for olanzapine. The $7 \mathrm{ng} / \mathrm{mL}$ reported for olanzapine were thus lower than expected $\mathrm{Cmin}$. On the other hand, the metabolite to parent compound ratio was 0.29 and thus as expected (see $>$ Table 6 ). The 


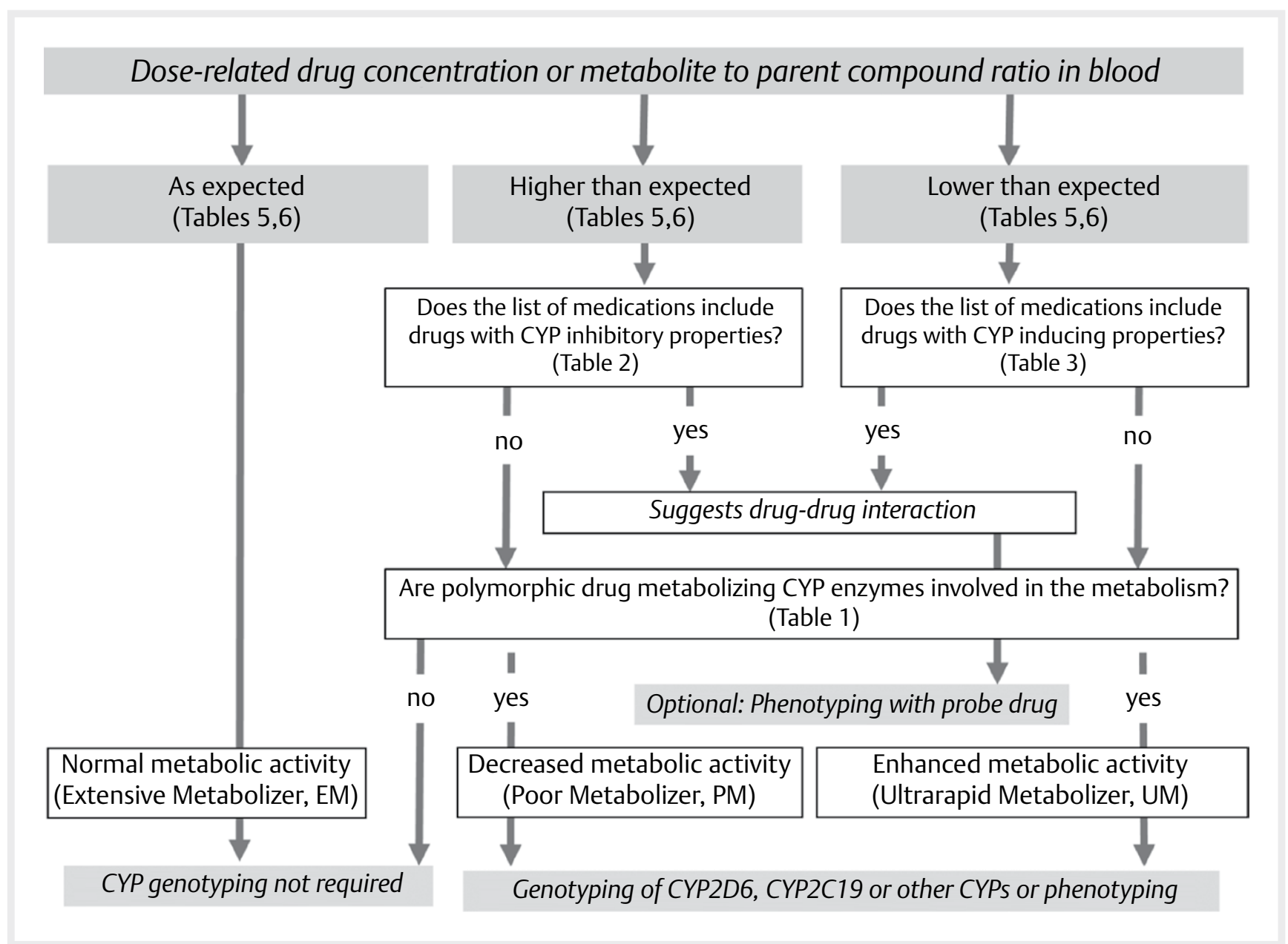

- Fig. 6 Algorithm for using genotyping of cytochrome P450 (CYP) enzymes and phenotyping with a probe drug in combination with TDM.

patient was a moderate smoker. $>$ Table 3 indicates that smoking induces CYP1A2 and $>$ Table 1 shows that olanzapine is a substrate of CYP1A2. Lower-than-expected concentrations of olanzapine and normal metabolite to parent compound ratios can be best explained by adherence problems. However, the effect of enhanced olanzapine degradation by smoking is another possible explanation.

\section{Recommendation}

Poor response is plausible due to the low drug concentration in blood. Patient adherence has to be addressed and verified. In case of complete adherence, a dose increase can be helpful.

The three cases demonstrate how information given in the - Tables 1-6 can be used for interpretation of laboratory data to draw valid conclusions and give substantial recommendations for rational pharmacotherapy. Nevertheless, interpretation of TDM results relies on complex quantitative relationships. Therefore, training in clinical neuropsychopharmacology, pharmacokinetics and the application of TDM information is essential. Regular conferences with discussion of the interpretation of real cases are most helpful for learning. It is also recommended that junior psychiatrists interpret the results under supervision of an expert.

\subsection{Pharmacogenetic tests in addition to TDM}

When a pharmacogenetic test is carried out prior to prescribing a particular drug under defined circumstances [247, 248, 332, $568,569,632-634,658,1135,1229]$ concentrations outside the therapeutic or dose-related reference range may be avoided when this is due to gene polymorphisms that give rise to poor/ultrarapid metabolizers (pharmacokinetic level). Situations and cases where pharmacogenetic tests could be combined with TDM are explained in > Fig. 6. In agreement with recommendations of the German Commission Genetic Testing (GeKO) and the Clinical Pharmacogenetics Implementation Consortium [515, 517, 1229] as well as regulatory administrations such as the FDA and EMA the most important indications for genotyping of drug metabolizing enzymes in combination with TDM are the following:

- A priori genotyping when a drug is characterized by a small therapeutic index with a risk of toxicity in the case of a genetically impaired metabolism. 


\section{Initial prescription or change of dose}

I

Blood withdrawal under steady-state at the time of minimal drug concentrations

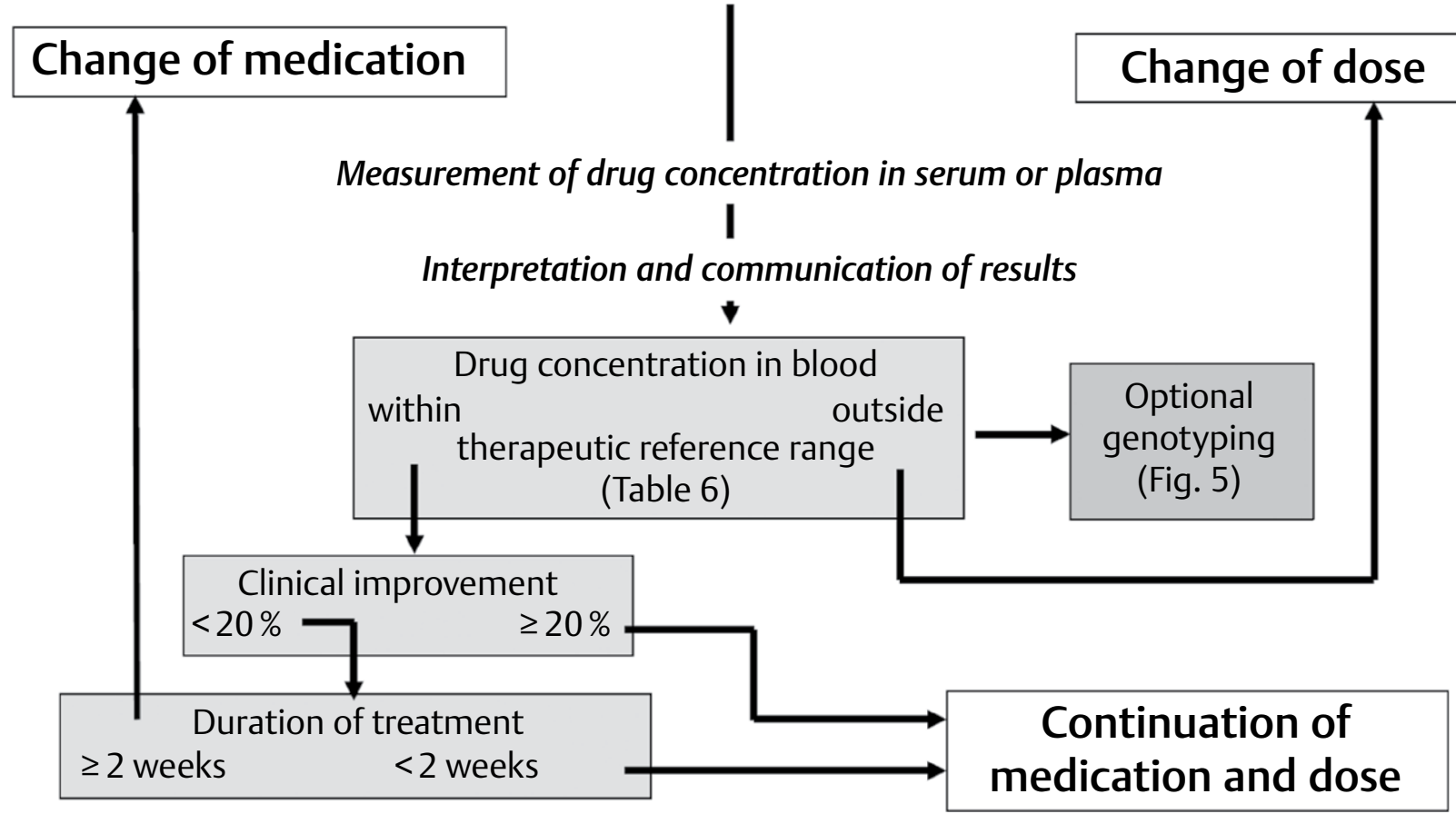

Fig. 7 TDM-guided dose titration for treatment with mood stabilizing, antidepressant, antipsychotic or antiepileptic drugs. Clinical decision making has to consider steady-state concentration of the drug in blood, clinical improvement and duration of treatment. $94 \%$ of the steady-state is reached after four elimination half-lives of the drug or active metabolite. Decisons to change the dose or the medication can be necessary in case of adverse drug reactions. This was not considered in this scheme.

- A priori genotyping when the patient is treated with a substrate with a wide interindividual variability in metabolism and considerable risk of toxicity in case of overdosing, e. g., tricyclic antidepressants.

- Post hoc genotyping when the patient presents unusual plasma concentrations of the drug or its metabolite(s) to define metabolic status prior to administering other drugs e. g., codeine in the case of ultrarapid metabolizers (see warnings in the drug labels for codeine for ultrarapid metabolizers [331]).

In a patient who is genotyped as a PM or UM, the medication needs not automatically be replaced, but the dose may be adapted, using TDM and clinical judgement.

Pharmacogenetic testing on a pharmacodynamic level is not recommended yet in clinical practice, except for carbamazepine [354].

Commercially available test batteries for detection of pharmacokinetic and pharmacodynamic gene variants are currently marketed, but evidence at present does not allow recommending their uncritical integration into everyday clinical practice.

There is a definite need for further research in large multi-centre trials.

\subsection{Clinical decision making}

A TDM result is a guide to proper dosing of the individual patient ( $\triangleright$ Fig. 7). The physician has to be aware that, under optimal conditions, reporting of results with inclusion of dose recommendations and other comments by the laboratory is based on the best available evidence [518, 520]. The laboratory, however, has only restricted knowledge of the clinical situation. On the other hand, most treating physicians have limited pharmacokinetic knowledge. Therefore, it is essential to acknowledge that optimal TDM is an interdisciplinary task requiring close communication between laboratory and clinical experts.

If the measured drug concentration is within the therapeutic reference range, a change of the dose is, of course, only recommended if clinical reasons, such as adverse drug reactions or nonresponse, clearly justify such a decision. The treating physician has to decide whether the treatment strategy is to be changed or not. On the other hand, when the advice given on the TDM report is not followed, the reason must be substantiated to allow evaluation of the treating physician's decision should the patient come to harm. Recommendations for such an evaluation in a court of law have been published by the TDM-AGNP group [1345].

In patients with known abnormally rapid elimination it may be useful to prescribe a dose above the maximal recommended dose, 
since such patients can exhibit drug concentrations below the reference range under standard doses. However, the medication should be changed if the patient exhibited sufficiently high drug concentrations for a sufficiently long treatment period, i.e., for at least 2 weeks, and did not improve by at least $20 \%$. Another option can be the use of a drug that is not metabolized via CYP, like the antidepressant drug milnacipran or the antipsychotic drug amisulpride.

When adverse drug reactions are associated with clinical improvement under recommended doses, measurement of the drug concentrations in blood may clarify if adverse drug reactions are related to exceedingly high drug levels in the blood. In this situation, the dose can be decreased, normally without risk of loss of action.

For the treatment with antidepressant, antipsychotic or mood stabilizing drugs, there is good evidence that clinical non-improvement at week 2 is highly predictive for later treatment failure $[196,239,615,696,1130,1131,1162]$. For dose titration with antidepressant or antipsychotic drugs we therefore recommend to include symptom rating by the treating physician [239] at baseline and at week 2 in addition to drug concentration measurements. - Fig. 7 summarizes the above recommendations in a flow chart.

When further drug concentration measurements in blood are recommended after a modification of the dose or after prescription of a comedication that is known to inhibit or enhance the metabolism of the drug to be measured, the next TDM should be delayed until steady-state conditions are reached again. For this, the terminal elimination half-life ( $\mathrm{t} 1 / 2)$ of the drug has to be considered ( $\triangleright$ Table 4). Finally, if the patient has improved under a drug concentration below the reference range, (gradual) termination of the medication should be considered, because the medication may serve as a placebo only while still carrying the risk of adverse drug reactions and being costly.

\subsection{Cost-effectiveness of TDM}

TDM has been shown to be cost-effective (for review see [1204]). For tricyclic antidepressant drugs, this was evidenced as a reduction of the intoxication risk $[168,961,962]$. When patients were pre-monitored by administration of test doses of amitriptyline or nortriptyline for an estimation of the elimination rate and the elimination half-life to calculate the dose required to attain therapeutically effective steady-state concentrations of the drug in blood [159], the pharmacokinetic dosing decreased costs markedly [1089]. The pharmacokinetically dosed patients were discharged from hospital six days earlier and returned to work 55 days earlier than the empirically dosed patients. For SSRI, Lundmark and coworkers [734] observed in a sample of 127 elderly outpatients that the introduction of TDM led to dose reduction in 38 cases resulting in a reduction of drug costs by $16 \%$. A large cost reduction was reported for citalopram: TDM markedly decreased the duration of hospitalization [894]. In this study on inpatients, TDM-guided pharmacotherapy, yielding sufficiently high citalopram serum concentrations ( $>50 \mathrm{ng} / \mathrm{ml}$ ), decreased the stay in the hospital by 23 days compared to a patient group with subtherapeutic citalopram concentrations. Drug concentrations below $50 \mathrm{ng} / \mathrm{mL}$ on day 7 of treatment were highly predictive for later treatment failure [895]. Similar findings have been reported for depressed patients treated with venlafaxine [1129]. Moreover, it can be assumed that TDM has the potential to reduce relapse rates. Given that TDM detects non-ad- herence to medication before re-hospitalization, TDM is highly cost-effective. One day in the hospital is 4-16 times more expensive than a single drug concentration measurement in the laboratory. In summary, due to the potential of improving adherence, acceleration of clinical improvement or decrease of hospitalisation length by TDM, a marked cost effect can be expected by TDM. More studies on the cost effectiveness of TDM, however, are required.

\section{Conclusions and Perspectives}

This second update of the AGNP guidelines describes the practice of TDM to promote the appropriate use of TDM in psychiatry and neurology. When applied adequately, TDM is an excellent tool of precision medicine to optimize the pharmacotherapy of individual patients. During the past decades, knowledge on the metabolic fate and actions of drugs in the human body has markedly advanced. However, there is a gap between the availability of knowledge in pharmacology and its utilization in health care [518, 1094]. TDM bridges this gap. For this update, special attention was given to methods that enable pharmacokinetic characterization of the patient. Combining information related to therapeutic reference ranges, dose-related reference ranges, metabolite to parent compound ratios as well as properties of administered drugs like CYP substrate, inhibitor and inducer specificities and finally genotypes of CYP enzymes and drug transporters allows to recognize and document individual characteristics in the pharmacodynamics and -kinetics of neuropsychiatric drugs. The information can be used for rational dose corrections to optimize efficacy and tolerability of these medications as well as treatment costs. In spite of objective advances with respect to the use of TDM in everyday clinical practice, quality improvement of TDM must still be continuously addressed. There is also a need for inclusion of pharmacokinetic measurements during clinical trials of drug development. It is a major shortcoming that data on drug concentrations in blood that are optimal for attaining the highest probability of clinical response are not legally required for the registration of medications. Product information should be supplemented with TDM-related data. Last not least, teaching of these issues at a postgraduate level is necessary for psychiatry residents [86].

\section{Conflicts of Interest}

Christoph Hiemke has received speaker's and consultancy fees from Janssen, Stada, Servier. He is managing director of the psiac GmbH (www.psiac. de) which provides an internet based drug-drug interaction program. Pierre Baumann has received speaker's or consultancy fees from almost all pharmaceutical companies selling psychotropic drugs in Switzerland. Niels Bergemann received speaker's or consultancy fees and/or educational grants from AstraZeneca, Bristol-Myers Squibb, Janssen, Lilly, Otsuka, Pfizer, Servier. Andreas Conca has served as a consultant for Lilly, Bristol-Myers Squibb, Pfizer. He has served on the speakers' bureau of Lilly, BMS, Astra Zeneca, Lundbeck, Italfarma, Janssen. Gabriel Eckermann has received speaker's fees from almost all pharmaceutical companies selling psychotropic drugs in Germany. He is shareholder of the psiac $\mathrm{GmbH}$ (www.psiac.de), which provides an internet based drug-drug interaction program. Karin Egberts participated in performing clinical trials for AstraZeneca, Janssen-Cilag, Lilly, Shire and has received research grants pertaining to pharmacovigilance in children and 
adolescents from the German Federal Institute for Drugs and Medical Devices. Ursula Havemann-Reinecke has received speaker's and consultancy fees and unrestricted educational grants from AstraZeneca, Bristol-Myers Squibb, Cephalon, Essex, Janssen Cilag, Lundbeck, Pfizer, Schering-Plough, Wyeth. Ekkehard Haen is chairman and managing director of the AGATE (www.amuep-agate.de) that supports reasonable and economic drug therapy. He is shareholder of the psiac $\mathrm{GmbH}$ (www.psiac.de), which provides an internet based drug-drug interaction program. Manfred Gerlach has received research grants pertaining to pharmacovigilance in children and adolescents from the German Federal Institute for Drugs and Medical Devices. He has also received royalties from Springer Vienna for editing a German and English textbook on child and adolescent psychiatry. Gerhard Gründer has served as a consultant for Astra Zeneca, Bristol-Myers Squibb, Eli Lilly, Johnson \& Johnson, Otsuka. He has served on the speakers' bureau of Astra Zeneca, Bristol-Myers Squibb, Eli Lilly, Janssen Cilag, Otsuka, Pfizer, Servier, Wyeth. He has received grant support from Alkermes, Bristol-Myers Squibb, Eli Lilly, Johnson \& Johnson. He is co-founder of Pharma-Image - Molecular Imaging Technologies $\mathrm{GmbH}$. Eveline Jaquenoud is a member of mediQ (www.mediq.ch) which provides an internet based drug-drug interaction program for psychiatry. Gerd Laux has received speaker's or consultancy fees or unrestricted educational grants from AstraZeneca, Bayer, Eli Lilly, Lundbeck, Merz, Pfizer, Servier, Wyeth. Thomas Messer has received speaker's or consultancy fees or unrestricted educational grants from Eli Lilly, BristolMyers Squibb, Janssen, Servier, Pfizer, Lundbeck, Bayer Vital Health Care. Matthias J. Müller has received speaker's or consultancy fees from Janssen, Lundbeck, Servier. Bruno Pfuhlmann has received speaker's or consultancy fees from AstraZeneca, Janssen, Pfizer. Sven Ulrich is an employee of Ariston Pharma GmbH, Berlin, Germany. Gerald Zernig has received speaker's or consultancy fees or educational grants from AlcaSynn, AstraZeneca, BioRad, Bristol-Myers Squibb, Eli Lilly, Lundbeck, Mundipharma, Novartis, Pfizer, Wyeth. Hans Willi Clement, Jürgen Deckert, Katharina Domschke, Christine Greiner, Gudrun Hefner, Renate Helmer, Ger Janssen, Rainold Mössner, Michael Paulzen, Peter Riederer, Alois Saria, Bernd Schoppek, Georgios Schoretsanitis, Markus Schwarz, Margarete Silva Gracia, Benedikt Stegmann, Werner Steimer, Julia C. Stingl, Manfred Uhr, Stefan Unterecker and Roland Waschgler were not supported by pharmaceutical industry.

\section{References}

[1] Abanades S, van der Aart J, Barletta JA et al. Prediction of repeat-dose occupancy from single-dose data: Characterisation of the relationship between plasma pharmacokinetics and brain target occupancy. J Cereb Blood Flow Metab 2011; 31: 944-952

[2] Abdelbary A, Bendas ER, Ramadan AA et al. Pharmaceutical and pharmacokinetic evaluation of a novel fast dissolving film formulation of flupentixol dihydrochloride. AAPS PharmSciTech 2014; 15 : $1603-1610$

[3] Abernethy DR, Greenblatt D], Shader RI. Imipramine and desipramine disposition in the elderly. J Pharmacol Exp Ther 1985; 232: 183-188

[4] Adam K, Oswald I. Effects of lormetazepam and of flurazepam on sleep. Br J Clin Pharmacol 1984; 17: 531-538

[5] Adamiak U, Kaldonska M, Klodowska-Duda G et al. Pharmacokineticpharmacodynamic modeling of levodopa in patients with advanced Parkinson disease. Clin Neuropharmacol 2010; 33: 135-141

[6] Addington D. Best practices: Improving quality of care for patients with first-episode psychosis. Psychiatr Serv 2009; 60: 1164-1166

[7] Adjei A, Teuscher NS, Kupper RJ et al. Single-dose pharmacokinetics of methylphenidate extended-release multiple layer beads administered as intact capsule or sprinkles versus methylphenidate immediaterelease tablets (Ritalin((R))) in healthy adult volunteers. | Child Adolesc Psychopharmacol 2014; 24: 570-578
[8] Adli M, Baethge C, Heinz A et al. Is dose escalation of antidepressants a rational strategy after a medium-dose treatment has failed? A systematic review. Eur Arch Psychiatry Clin Neurosci 2005; 255: 387-400

[9] Aichhorn W, Marksteiner J, Walch T et al. Influence of age, gender, body weight and valproate comedication on quetiapine plasma concentrations. Int Clin Psychopharmacol 2006; 21: 81-85

[10] Aichhorn W, Weiss U, Marksteiner ] et al. Influence of age and gender on risperidone plasma concentrations. J Psychopharmacol 2005; 19: 395-401

[11] Aichhorn W, Whitworth AB, Weiss EM et al. Second-generation antipsychotics: Is there evidence for sex differences in pharmacokinetic and adverse effect profiles? Drug Saf 2006; 29: 587-598

[12] Akamine $\mathrm{Y}$, Uehara $\mathrm{H}$, Miura $\mathrm{M}$ et al. Multiple inductive effects of carbamazepine on combined therapy with paliperidone and amlodipine. J Clin Pharm Ther 2015; 40: 480-482

[13] Akerblad AC, Bengtsson F, Ekselius L et al. Effects of an educational compliance enhancement programme and therapeutic drug monitoring on treatment adherence in depressed patients managed by general practitioners. Int Clin Psychopharmacol 2003; 18: 347-354

[14] Akerblad AC, Bengtsson F, Holgersson M et al. Identification of primary care patients at risk of nonadherence to antidepressant treatment. Patient Prefer Adherence 2008; 2: 379-386

[15] Aklillu E, Djordjevic N, Carrillo JA et al. High CYP2A6 enzyme activity as measured by a caffeine test and unique distribution of CYP2A6 variant alleles in Ethiopian population. OMICS 2014; 18: 446-453

[16] Aklillu E, Kalow W, Endrenyi L et al. CYP2D6 and DRD2 genes differentially impact pharmacodynamic sensitivity and time course of prolactin response to perphenazine. Pharmacogenet Genomics 2007; 17: 989-993

[17] Akutsu T, Kobayashi K, Sakurada K et al. Identification of human cytochrome p450 isozymes involved in diphenhydramine $\mathrm{N}$-demethylation. Drug Metab Dispos 2007; 35: 72-78

[18] Al-janabi I, Arranz M], Blakemore Al et al. Association study of serotonergic gene variants with antipsychotic-induced adverse reactions. Psychiatr Genet 2009; 19: 305-311

[19] Al Hadithy AF, Ivanova SA, Pechlivanoglou P et al. Tardive dyskinesia and DRD3, HTR2A and HTR2C gene polymorphisms in Russian psychiatric inpatients from Siberia. Prog Neuropsychopharmacol Biol Psychiatry 2009; 33: 475-481

[20] Alderman ], Wolkow R, Fogel IM. Drug concentration monitoring with tolerability and efficacy assessments during open-label, long-term sertraline treatment of children and adolescents. J Child Adolesc Psychopharmacol 2006; 16: 117-129

[21] Alexanderson B. Prediction of steady-state plasma levels of nortriptyline from single oral dose kinetics: A study in twins. Eur J Clin Pharmacol 1973; 6: 44-53

[22] Allen MD, Greenblatt D], Harmatz JS et al. Desmethyldiazepam kinetics in the elderly after oral prazepam. Clin Pharmacol Ther 1980; 28: 196-202

[23] Allen MH, Hirschfeld RM, Wozniak PJ et al. Linear relationship of valproate serum concentration to response and optimal serum levels for acute mania. Am J Psychiatry 2006; 163: 272-275

[24] Allqvist A, Miura J, Bertilsson L et al. Inhibition of CYP3A4 and CYP3A5 catalyzed metabolism of alprazolam and quinine by ketoconazole as racemate and four different enantiomers. Eur J Clin Pharmacol 2007; 63: $173-179$

[25] Altamura AC, Moliterno D, Paletta S et al. Effect of quetiapine and norquetiapine on anxiety and depression in major psychoses using a pharmacokinetic approach: A prospective observational study. Clin Drug Investig 2012; 32: 213-219 
[26] Altamura AC, Moliterno D, Paletta S et al. Understanding the pharmacokinetics of anxiolytic drugs. Expert Opin Drug Metab Toxicol 2013; 9: 423-440

[27] Altamura AC, Moro AR, Percudani M. Clinical pharmacokinetics of fluoxetine. Clin Pharmacokinet 1994; 26: 201-214

[28] Altamura AC, Sassella F, Santini A et al. Intramuscular preparations of antipsychotics: uses and relevance in clinical practice. Drugs 2003; 63 : 493-512

[29] Althaus M, Retzow A, Castell JV et al. In vitro identification of the cytochrome $\mathrm{P} 450$ isoform responsible for the metabolism of alpha-dihydroergocryptine. Xenobiotica 2000; 30: 1033-1045

[30] Aman MG, Vinks AA, Remmerie B et al. Plasma pharmacokinetic characteristics of risperidone and their relationship to saliva concentrations in children with psychiatric or neurodevelopmental disorders. Clin Ther 2007; 29: 1476-1486

[31] Amdisen A. Serum level monitoring and clinical pharmacokinetics of lithium. Clin Pharmacokinet 1977; 2: 73-92

[32] American Geriatrics Society Beers Criteria Update Expert P. American Geriatrics Society updated Beers Criteria for potentially inappropriate medication use in older adults. J Am Geriatr Soc 2012; 60: 616-631

[33] Anderson D, Reed S, Lintemoot J et al. A first look at duloxetine (Cymbalta) in a postmortem laboratory. J Anal Toxicol 2006; 30: 576-580

[34] Anderson GD. Pharmacogenetics and enzyme induction/inhibition properties of antiepileptic drugs. Neurology 2004; 63: S3-S8

[35] Anderson IM, Ferrier IN, Baldwin RC et al. Evidence-based guidelines for treating depressive disorders with antidepressants: a revision of the 2000 British Association for Psychopharmacology guidelines. J Psychopharmacol 2008; 22: 343-396

[36] Ansermot N, Albayrak O, Schlapfer J et al. Substitution of (R,S)-methadone by (R)-methadone: Impact on QTc interval. Arch Intern Med 2010; 170: 529-536

[37] Ansermot N, Brawand-Amey M, Eap CB. Simultaneous quantification of selective serotonin reuptake inhibitors and metabolites in human plasma by liquid chromatography-electrospray mass spectrometry for therapeutic drug monitoring. J Chromatogr B Analyt Technol Biomed Life Sci 2012; 885-886: 117-130

[38] Aoki FY, Sitar DS. Clinical pharmacokinetics of amantadine hydrochloride. Clin Pharmacokinet 1988; 14: 35-51

[39] Aps JK, Martens LC. Review: The physiology of saliva and transfer of drugs into saliva. Forensic Sci Int 2005; 150: 119-131

[40] Arakawa R, Ito H, Takano A et al. Dose-finding study of paliperidone ER based on striatal and extrastriatal dopamine D2 receptor occupancy in patients with schizophrenia. Psychopharmacology (Berl) 2008; 197: 229-235

[41] Arango C, Bombin I, Gonzalez-Salvador T et al. Randomised clinical trial comparing oral versus depot formulations of zuclopenthixol in patients with schizophrenia and previous violence. Eur Psychiatry 2006; $21: 34-40$

[42] Aravagiri M, Marder SR, Yuwiler A et al. Distribution of fluphenazine and its metabolites in brain regions and other tissues of the rat. Neuropsychopharmacology 1995; 13: 235-247

[43] Aravagiri M, Teper Y, Marder SR. Pharmacokinetics and tissue distribution of olanzapine in rats. Biopharm Drug Dispos 1999; 20: 369-377

[44] Aravagiri M, Yuwiler A, Marder SR. Distribution after repeated oral administration of different dose levels of risperidone and 9-hydroxyrisperidone in the brain and other tissues of rat. Psychopharmacology (Berl) 1998; 139: 356-363

[45] Arbus C, Benyamina A, Llorca PM et al. Characterization of human cytochrome P450 enzymes involved in the metabolism of cyamemazine. Eur J Pharm Sci 2007; 32: 357-366
[46] Areberg J, Luntang-Jensen M, Sogaard B et al. Occupancy of the serotonin transporter after administration of Lu AA21004 and its relation to plasma concentration in healthy subjects. Basic Clin Pharmacol Toxicol 2012; 110: 401-404

[47] Areberg J, Petersen KB, Chen G et al. Population pharmacokinetic meta-analysis of vortioxetine in healthy individuals. Basic Clin Pharmacol Toxicol 2014; 115: 552-559

[48] Armijo JA, Cuadrado A, Bravo J et al. Vigabatrin serum concentration to dosage ratio: Influence of age and associated antiepileptic drugs. Ther Drug Monit 1997; 19: 491-498

[49] Aronson JK. Compliance, concordance, adherence. $\mathrm{Br}$ J Clin Pharmacol 2007; 63: 383-384

[50] Aronson JK, Ferner RE. The law of mass action and the pharmacological concentration-effect curve: Resolving the paradox of apparently non-dose-related adverse drug reactions. Br J Clin Pharmacol 2016; 81: 56-61

[51] Asberg M, Cronholm B, Sjoqvist F et al. Correlation of subjective side effects with plasma concentrations of nortriptyline. Br Med J 1970; 4: $18-21$

[52] Asberg M, Cronholm B, Sjoqvist F et al. Relationship between plasma level and therapeutic effect of nortriptyline. Br Med J 1971; 3: 331-334

[53] Ashby M, Fleming B, Wood M et al. Plasma morphine and glucuronide (M3G and M6G) concentrations in hospice inpatients. J Pain Symptom Manage 1997; 14: 157-167

[54] Athanasoulia AP, Sievers C, Ising M et al. Polymorphisms of the drug transporter gene $A B C B 1$ predict side effects of treatment with cabergoline in patients with PRL adenomas. Eur J Endocrinol 2012; 167: $327-335$

[55] Bach MV, Coutts RT, Baker GB. Involvement of CYP2D6 in the in vitro metabolism of amphetamine, two $\mathrm{N}$-alkylamphetamines and their 4-methoxylated derivatives. Xenobiotica 1999; 29: 719-732

[56] Bachmann C], Haberhausen M, Heinzel-Gutenbrunner M et al. Large intraindividual variability of olanzapine serum concentrations in adolescent patients. Ther Drug Monit 2008; 30: 108-112

[57] Bachmann C], Rieger-Gies A, Heinzel-Gutenbrunner M et al. Large variability of aripiprazole and dehydroaripiprazole serum concentrations in adolescent patients with schizophrenia. Ther Drug Monit 2008; 30: 462-466

[58] Bachus R, Bickel U, Thomsen T et al. The O-demethylation of the antidementia drug galanthamine is catalysed by cytochrome P450 2D6. Pharmacogenetics 1999; 9: 661-668

[59] Backman JT, Filppula AM, Niemi M et al. Role of Cytochrome P450 2C8 in Drug Metabolism and Interactions. Pharmacol Rev 2016; 68: 168-241

[60] Backman JT, Olkkola KT, Ojala M et al. Concentrations and effects of oral midazolam are greatly reduced in patients treated with carbamazepine or phenytoin. Epilepsia 1996; 37: 253-257

[61] Bagli M, Hoflich G, Rao ML et al. Bioequivalence and absolute bioavailability of oblong and coated levomepromazine tablets in CYP2D6 phenotyped subjects. Int J. Clin Pharmacol Ther 1995; 33: 646-652

[62] Bagli M, Rao ML, Hoflich G et al. Pharmacokinetics of chlorprothixene after single intravenous and oral administration of three galenic preparations. Arzneimittelforschung 1996; 46: 247-250

[63] Baird-Bellaire S, Behrle JA, Parker VD et al. An open-label, single-dose, parallel-group study of the effects of chronic hepatic impairment on the safety and pharmacokinetics of desvenlafaxine. Clin Ther 2013; 35: 782-794

[64] Baker GB, Urichuk LJ, McKenna KF et al. Metabolism of monoamine oxidase inhibitors. Cell Mol Neurobiol 1999; 19: 411-426 
[65] Bakken GV, Rudberg I, Christensen $\mathrm{H}$ et al. Metabolism of quetiapine by CYP3A4 and CYP3A5 in presence or absence of cytochrome B5. Drug Metab Dispos 2009; 37: 254-258

[66] Bakken GV, Rudberg I, Molden E et al. Pharmacokinetic variability of quetiapine and the active metabolite $\mathrm{N}$-desalkylquetiapine in psychiatric patients. Ther Drug Monit 2011; 33: 222-226

[67] Balant-Gorgia AE, Eisele R, Aeschlimann JM et al. Plasma flupentixol concentrations and clinical response in acute schizophrenia. Ther Drug Monit 1985; 7: 411-414

[68] Balant LP, Balant-Gorgia AE, Eisele R et al. Clinical and pharmacokinetic evaluation of zuclopenthixol acetate in Viscoleo. Pharmacopsychiatry 1989; 22: 250-254

[69] Baldinger P, Kranz GS, Haeusler D et al. Regional differences in SERT occupancy after acute and prolonged SSRI intake investigated by brain PET. Neuroimage 2014; 88: 252-262

[70] Barbhaiya RH, Shukla UA, Pfeffer M et al. Disposition kinetics of buspirone in patients with renal or hepatic impairment after administration of single and multiple doses. Eur J Clin Pharmacol 1994; 46: $41-47$

[71] Bareggi SR, Bianchi L, Cavallaro R et al. Citalopram concentrations and response in obsessive-compulsive disorder. Preliminary results. CNS Drugs 2004; 18: 329-335

[72] Barry M, Mulcahy F, Merry C et al. Pharmacokinetics and potential interactions amongst antiretroviral agents used to treat patients with HIV infection. Clin Pharmacokinet 1999; 36: 289-304

[73] Barski OA, Tipparaju SM, Bhatnagar A. The aldo-keto reductase superfamily and its role in drug metabolism and detoxification. Drug Metab Rev 2008; 40: 553-624

[74] Baruzzi A, Bordo B, Bossi L et al. Plasma levels of di-no-propylacetate and clonazepam in epileptic patients. Int J Clin Pharmacol Biopharm 1977; 15: 403-408

[75] Bates DW, Gawande AA. Improving safety with information technology. N Engl J Med 2003; 348: 2526-2534

[76] Batty KT, Davis TM, llett KF et al. The effect of ciprofloxacin on theophylline pharmacokinetics in healthy subjects. $\mathrm{Br}$ J Clin Pharmacol 1995; 39: 305-311

[77] Bauer LA. Applied Clinical Pharmacokinetics. 3rd ed.New York: McGraw-Hill Education; 2014

[78] Bauer S, David Rudd G, Mylius V et al. Lacosamide intoxication in attempted suicide. Epilepsy Behav 2010; 17: 549-551

[79] Baumann P. Pharmacokinetic-pharmacodynamic relationship of the selective serotonin reuptake inhibitors. Clin Pharmacokinet 1996; 31 : 444-469

[80] Baumann P, Barbe R, Vabre-Bogdalova A et al. Epileptiform seizure after sertraline treatment in an adolescent experiencing obsessivecompulsive disorder and presenting a rare pharmacogenetic status. J Clin Psychopharmacol 2006; 26: 679-681

[81] Baumann P, Hiemke C. Central nervous system drugs.In: Metabolism of drugs and other xenobiotics. 1st ed. Anzenbacher P, Zanger UM. (eds)Wiley-VCH; 2012: Chapter 11: 301-329

[82] Baumann P, Hiemke C, Ulrich S et al. The AGNP-TDM expert group consensus guidelines: Therapeutic drug monitoring in psychiatry. Pharmacopsychiatry 2004; 37: 243-265

[83] Baumann P, Kirchherr H, Berney P et al. Flupentixol: Relevance of stereoselective therapeutic drug monitoring. Psychopharmacology (Berl) 2012; 221: 719-720

[84] Baumann P, Meyer JW, Amey M et al. Dextromethorphan and mephenytoin phenotyping of patients treated with thioridazine or amitriptyline. Ther Drug Monit 1992; 14: 1-8

[85] Baumann P, Rougemont M, Corruble E et al. Recommendations for therapeutic monitoring of antidepressants. Rev Med Suisse 2013; 9: $577-586$
[86] Baumann P, Spies M, Moller H] et al. A proposal for a psychopharmacology-pharmacotherapy catalogue of learning objectives and a curriculum in Europe. World J Biol Psychiatry 2017; 18: 29-38

[87] Baumann P, Tinguely D, Koeb L et al. On the relationship between free plasma and saliva amitriptyline and nortriptyline. Int Pharmacopsychiatry 1982; 17: 136-146

[88] Baumann P, Zullino DF, Eap CB. Enantiomers' potential in psychopharmacology-a critical analysis with special emphasis on the antidepressant escitalopram. Eur Neuropsychopharmacol 2002; 12: 433-444

[89] Bazire S. Psychotropic Drug Directory. 2016 Aberdeen: Lloyd-Reinhold Publications Ltd

[90] Beasley CM Jr., Stauffer VL, Liu-Seifert $\mathrm{H}$ et al. All-cause treatment discontinuation in schizophrenia during treatment with olanzapine relative to other antipsychotics: An integrated analysis. J Clin Psychopharmacol 2007; 27: 252-258

[91] Bech P, Gex-Fabry M, Aubry JM et al. Olanzapine plasma level in relation to antimanic effect in the acute therapy of manic states. Nord J Psychiatry 2006; 60: 181-182

[92] Becquemont L, Mouajjah S, Escaffre O et al. Cytochrome P-450 3A4 and 2C8 are involved in zopiclone metabolism. Drug Metab Dispos 1999; 27: 1068-1073

[93] Beedham C, Miceli J], Obach RS. Ziprasidone metabolism, aldehyde oxidase, and clinical implications. J Clin Psychopharmacol 2003; 23 : 229-232

[94] Benedetti MS, Whomsley R, Poggesi I et al. Drug metabolism and pharmacokinetics. Drug Metab Rev 2009; 41: 344-390

[95] Benetton SA, Fang C, Yang YO et al. P450 phenotyping of the metabolism of selegiline to desmethylselegiline and methamphetamine. Drug Metab Pharmacokinet 2007; 22: 78-87

[96] Bengtsson F. Therapeutic drug monitoring of psychotropic drugs. TDM "nouveau". Ther Drug Monit 2004; 26: 145-151

[97] Bennett JP Jr., Piercey MF. Pramipexole-a new dopamine agonist for the treatment of Parkinson's disease. J Neurol Sci 1999; 163: 25-31

[98] Bentue-Ferrer D, Tribut O, Verdier MC. Therapeutic drug monitoring of ethosuximide. Therapie 2012; 67: 391-396

[99] Bentue-Ferrer D, Tribut O, Verdier MC. Therapeutic drug monitoring of lacosamide. Therapie 2012; 67: 151-155

[100] Bentue-Ferrer D, Tribut O, Verdier MC. Therapeutic drug monitoring of rufinamide. Therapie 2012; 67: 161-165

[101] Bergemann N, Frick A, Parzer P et al. Olanzapine plasma concentration, average daily dose, and interaction with co-medication in schizophrenic patients. Pharmacopsychiatry 2004; 37: 63-68

[102] Bergemann N, Kopitz J, Kress KR et al. Plasma amisulpride levels in schizophrenia or schizoaffective disorder. Eur Neuropsychopharmacol 2004; 14 : 245-250

[103] Bergemann N, Rommel F, Conca A. Therapeutisches drug-monitoring von psychopharmaka in der schwangerschaft. J Neurol Neurochir Psychiatr 2009; 10: 38-40

[104] Bergen AW, Michel M, Nishita D et al. Drug metabolizing enzyme and transporter gene variation, nicotine metabolism, prospective abstinence, and cigarette consumption. PLoS One 2015; 10 : e0126113

[105] Bergmann TK, Bathum L, Brosen K. Duplication of CYP2D6 predicts high clearance of desipramine but high clearance does not predict duplication of CYP2D6. Eur J Clin Pharmacol 2001; 57: 123-127

[106] Bergstrom RF, Cerimele BJ. Olanzapine in subjects with and without renal failure (data on file). Lilly Laboratory for Clinical Research. Eli Lilly and Co; 1996

[107] Berry D, Millington C. Analysis of pregabalin at therapeutic concentrations in human plasma/serum by reversed-phase HPLC. Ther Drug Monit 2005; 27: 451-456 
[108] Bertelsen KM, Venkatakrishnan K, Von Moltke LL et al. Apparent mechanism-based inhibition of human CYP2D6 in vitro by paroxetine: comparison with fluoxetine and quinidine. Drug Metab Dispos 2003; 31: 289-293

[109] Bertilsson L, Tomson T, Tybring G. Pharmacokinetics: Time-dependent changes--autoinduction of carbamazepine epoxidation. J Clin Pharmacol 1986; 26: 459-462

[110] Berwaerts ], Cleton A, Rossenu S et al. A comparison of serum prolactin concentrations after administration of paliperidone extended-release and risperidone tablets in patients with schizophrenia. J Psychopharmacol 2010; 24: 1011-1018

[111] Bet PM, Verbeek EC, Milaneschi Y et al. A common polymorphism in the $A B C B 1$ gene is associated with side effects of PGP-dependent antidepressants in a large naturalistic Dutch cohort. Pharmacogenomics J 2016; 16: 202-208

[112] Biernacka JM, Sangkuhl K, Jenkins G et al. The International SSRI Pharmacogenomics Consortium (ISPC): A genome-wide association study of antidepressant treatment response. Transl Psychiatry 2016; 6: e937

[113] Bigliani V, Mulligan RS, Acton PD et al. Striatal and temporal cortical D2/D3 receptor occupancy by olanzapine and sertindole in vivo: A [123I]epidepride single photon emission tomography (SPET) study. Psychopharmacology (Berl) 2000; 150: 132-140

[114] Bigos KL, Bies RR, Pollock BG et al. Genetic variation in CYP3A43 explains racial difference in olanzapine clearance. Mol Psychiatry 2011; 16: 620-625

[115] Birkenhager TK, van den Broek WW, Moleman P et al. Imipramine dose in relation to therapeutic plasma level: Are clinical trials using imipramine as a positive control flawed? Psychopharmacology (Berl) 2005; 181: 595-599

[116] Bishara D, Olofinjana O, Sparshatt A et al. Olanzapine: A systematic review and meta-regression of the relationships between dose, plasma concentration, receptor occupancy, and response. J Clin Psychopharmacol 2013; 33: 329-335

[117] Bjerkenstedt L, Flyckt L, Overo KF et al. Relationship between clinical effects, serum drug concentration and serotonin uptake inhibition in depressed patients treated with citalopram. A double-blind comparison of three dose levels. Eur J Clin Pharmacol 1985; 28 : 553-557

[118] Bjorndal N, Bjerre M, Gerlach J et al. High dosage haloperidol therapy in chronic schizophrenic patients: A double-blind study of clinical response, side effects, serum haloperidol, and serum prolactin. Psychopharmacology (Berl) 1980; 67: 17-23

[119] Bland TM, Haining RL, Tracy TS et al. CYP2C-catalyzed delta9-tetrahydrocannabinol metabolism: Kinetics, pharmacogenetics and interaction with phenytoin. Biochem Pharmacol 2005; 70: 10961103

[120] Blazquez A, Mas S, Plana MT et al. Plasma fluoxetine concentrations and clinical improvement in an adolescent sample diagnosed with major depressive disorder, obsessive-compulsive disorder, or generalized anxiety disorder. J Clin Psychopharmacol 2014; 34: 318-326

[121] Bockbrader HN. Clinical pharmacokinetics of gabapentin. Drugs Today 1995; 31: 613-619

[122] Bockbrader HN, Burger P, Knapp L et al. Population pharmacokinetics of pregabalin in healthy subjects and patients with chronic pain or partial seizures. Epilepsia 2011; 52: 248-257

[123] Bockbrader HN, Wesche D, Miller R et al. A comparison of the pharmacokinetics and pharmacodynamics of pregabalin and gabapentin. Clin Pharmacokinet 2010; 49: 661-669

[124] Boden R, Brandt L, Kieler $\mathrm{H}$ et al. Early non-adherence to medication and other risk factors for rehospitalization in schizophrenia and schizoaffective disorder. Schizophr Res 2011; 133: 36-41
[125] Boellner SW, Stark JG, Krishnan S et al. Pharmacokinetics of lisdexamfetamine dimesylate and its active metabolite, $d$-amphetamine, with increasing oral doses of lisdexamfetamine dimesylate in children with attention-deficit/hyperactivity disorder: A single-dose, randomized, open-label, crossover study. Clin Ther 2010; 32: 252-264

[126] Bogaards J], Hissink EM, Briggs M et al. Prediction of interindividual variation in drug plasma levels in vivo from individual enzyme kinetic data and physiologically based pharmacokinetic modeling. Eur J Pharm Sci 2000; 12: 117-124

[127] Boinpally R, Alcorn $\mathrm{H}$, Adams MH et al. Pharmacokinetics of vilazodone in patients with mild or moderate renal impairment. Clin Drug Investig 2013; 33: 199-206

[128] Boinpally R, Gad N, Gupta S et al. Influence of CYP3A4 induction/ inhibition on the pharmacokinetics of vilazodone in healthy subjects. Clin Ther 2014; 36: 1638-1649

[129] Bomsien S, Aderjan R, Mattern R et al. Effect of psychotropic medication on the in vitro metabolism of buprenorphine in human cDNA-expressed cytochrome P450 enzymes. Eur J Clin Pharmacol 2006; 62: 639-643

[130] Bond A, Seijas D, Dawling S et al. Systemic absorption and abuse liability of snorted flunitrazepam. Addiction 1994; 89: 821-830

[131] Bond AJ, Reed KD, Beavan P et al. After the randomised injectable opiate treatment trial: Post-trial investigation of slow-release oral morphine as an alternative opiate maintenance medication. Drug Alcohol Rev 2012; 31: 492-498

[132] Bondareva IB, Jelliffe RW, Gusev El et al. Population pharmacokinetic modelling of carbamazepine in epileptic elderly patients: implications for dosage. J Clin Pharm Ther 2006; 31: 211-221

[133] Bondolfi G, Morel F, Crettol S et al. Increased clozapine plasma concentrations and side effects induced by smoking cessation in 2 CYP1A2 genotyped patients. Ther Drug Monit 2005; 27: 539-543

[134] Bont L, Bosker HA, Brus F et al. Torsade de pointes after pipamperone intoxication. Pharm World Sci 1998; 20: 137

[135] Borgstrom L, Larsson H, Molander L. Pharmacokinetics of parenteral and oral melperone in man. Eur J Clin Pharmacol 1982; 23: 173-176

[136] Borys D], Setzer SC, Ling LJ et al. Acute fluoxetine overdose: a report of 234 cases. Am J Emerg Med 1992; 10: 115-120

[137] Bosse GM, Spiller HA, Collins AM. A fatal case of venlafaxine overdose. J Med Toxicol 2008; 4: 18-20

[138] Bowskill SV, Patel MX, Handley SA et al. Plasma amisulpride in relation to prescribed dose, clozapine augmentation, and other factors: data from a therapeutic drug monitoring service, 2002-2010. Hum Psychopharmacol 2012; 27: 507-513

[139] Bozik ME, Mather JL, Kramer WG et al. Safety, tolerability, and pharmacokinetics of KNS-760704 (dexpramipexole) in healthy adult subjects. J Clin Pharmacol 2011; 51: 1177-1185

[140] Brachtendorf L, Jetter A, Beckurts KT et al. Cytochrome P450 enzymes contributing to demethylation of maprotiline in man. Pharmacol Toxicol 2002; 90: 144-149

[141] Brahmi N, Kouraichi N, Abderrazek $\mathrm{H}$ et al. Clinical experience with carbamazepine overdose: Relationship between serum concentration and neurological severity. J Clin Psychopharmacol 2008; 28: 241-243

[142] Branch RA, Adedoyin A, Frye RF et al. In vivo modulation of CYP enzymes by quinidine and rifampin. Clin Pharmacol Ther 2000; 68: 401-411

[143] Brandl EJ, Kennedy JL, Müller D]. Pharmacogenetics of antipsychotics. Can J Psychiatry 2014; 59: 76-88

[144] Brandt C, Baumann P, Eckermann G et al. [Therapeutic drug monitoring in epileptology and psychiatry]. Nervenarzt 2008; 79: $167-174$ 
[145] Brandt C, May TW. Therapeutic drug monitoring of newer antiepileptic drugs. J Lab Med 2011; 35: 161-169

[146] Breitenstein B, Bruckl TM, Ising M et al. ABCB1 gene variants and antidepressant treatment outcome: A meta-analysis. Am J Med Genet B Neuropsychiatr Genet 2015; 168B: 274-283

[147] Breitenstein B, Scheuer S, Bruckl TM et al. Association of ABCB1 gene variants, plasma antidepressant concentration, and treatment response: Results from a randomized clinical study. J Psychiatr Res 2016; 73: 86-95

[148] Bressan RA, Erlandsson K, Jones HM et al. Is regionally selective D2/ D3 dopamine occupancy sufficient for atypical antipsychotic effect? an in vivo quantitative [123I]epidepride SPET study of amisulpridetreated patients. Am J Psychiatry 2003; 160: 1413-1420

[149] Bressolle F, Bres ], Faure-Jeantis A. Absolute bioavailability, rate of absorption, and dose proportionality of sulpiride in humans. J Pharm Sci 1992; 81: 26-32

[150] Breyer-Pfaff U. The metabolic fate of amitriptyline, nortriptyline and amitriptylinoxide in man. Drug Metab Rev 2004; 36: 723-746

[151] Breyer-Pfaff U, Brinkschulte M, Rein W et al. Prediction and evaluation criteria in perazine therapy of acute schizophrenics. Pharmacokinetic data. Pharmacopsychiatria 1983; 16: 160-165

[152] Breyer-Pfaff U, Nill K. Carbonyl reduction of naltrexone and dolasetron by oxidoreductases isolated from human liver cytosol. J Pharm Pharmacol 2004; 56: 1601-1606

[153] Breyer-Pfaff U, Nill K, Schied HW et al. Single-dose kinetics of the neuroleptic drug perazine in psychotic patients. Psychopharmacology (Berl) 1988; 95: 374-377

[154] Brockmoller J, Kirchheiner J, Schmider J et al. The impact of the CYP2D6 polymorphism on haloperidol pharmacokinetics and on the outcome of haloperidol treatment. Clin Pharmacol Ther 2002; 72: 438-452

[155] Brocks DR. Anticholinergic drugs used in Parkinson's disease: An overlooked class of drugs from a pharmacokinetic perspective. J Pharm Pharm Sci 1999; 2: 39-46

[156] Brosen K, Gram LF, Klysner R et al. Steady-state levels of imipramine and its metabolites: significance of dose-dependent kinetics. Eur J Clin Pharmacol 1986; 30: 43-49

[157] Brosen K, Klysner R, Gram LF et al. Steady-state concentrations of imipramine and its metabolites in relation to the sparteine/debrisoquine polymorphism. Eur J Clin Pharmacol 1986; 30: 679-684

[158] Brosen K, Naranjo CA. Review of pharmacokinetic and pharmacodynamic interaction studies with citalopram. Eur Neuropsychopharmacol 2001; 11: 275-283

[159] Browne JL, Perry PJ, Alexander B et al. Pharmacokinetic protocol for predicting plasma nortriptyline levels. J Clin Psychopharmacol 1983; 3: $351-356$

[160] Bruckl TM, Uhr M. ABCB1 genotyping in the treatment of depression. Pharmacogenomics 2016; 17: 2039-2069

[161] Bruhn O, Cascorbi I. Polymorphisms of the drug transporters ABCB1, $A B C G 2, A B C C 2$ and $A B C C 3$ and their impact on drug bioavailability and clinical relevance. Expert Opin Drug Metab Toxicol 2014; 10: 1337-1354

[162] Bruijn JA, Moleman P, Mulder PG et al. A double-blind, fixed blood-level study comparing mirtazapine with imipramine in depressed in-patients. Psychopharmacology (Berl) 1996; 127: 231-237

[163] Brunen S, Vincent PD, Baumann P et al. Therapeutic drug monitoring for drugs used in the treatment of substance-related disorders: literature review using a therapeutic drug monitoring appropriateness rating scale. Ther Drug Monit 2011; 33: 561-572

[164] Buckinx F, Rolland Y, Reginster JY et al. Burden of frailty in the elderly population: Perspectives for a public health challenge. Arch Public Health 2015; 73: 19
[165] Buckley NA, Dawson AH, Whyte IM et al. Greater toxicity in overdose of dothiepin than of other tricyclic antidepressants. Lancet 1994; 343: $159-162$

[166] Bundgaard C, Eneberg E, Sanchez C. P-glycoprotein differentially affects escitalopram, levomilnacipran, vilazodone and vortioxetine transport at the mouse blood-brain barrier in vivo. Neuropharmacology 2016; 103: 104-111

[167] Bunten H, Liang WJ, Pounder DJ et al. OPRM1 and CYP2B6 gene variants as risk factors in methadone-related deaths. Clin Pharmacol Ther 2010; 88: 383-389

[168] Burke MJ, Preskorn SH. Therapeutic drug monitoring of antidepressants: cost implications and relevance to clinical practice. Clin Pharmacokinet 1999; 37: 147-165

[169] Burns ML, Baftiu A, Opdal MS et al. Therapeutic drug monitoring of clobazam and its metabolite-impact of age and comedication on pharmacokinetic variability. Ther Drug Monit 2016; 38: 350-357

[170] Busardo FP, Jones AW. GHB pharmacology and toxicology: acute intoxication, concentrations in blood and urine in forensic cases and treatment of the withdrawal syndrome. Curr Neuropharmacol 2015; 13: $47-70$

[171] Byerly M], Thompson A, Carmody T et al. Validity of electronically monitored medication adherence and conventional adherence measures in schizophrenia. Psychiatr Serv 2007; 58: 844-847

[172] Cabaleiro T, Ochoa D, Lopez-Rodriguez R et al. Effect of polymorphisms on the pharmacokinetics, pharmacodynamics, and safety of risperidone in healthy volunteers. Hum Psychopharmacol 2014; 29: 459-469

[173] Caccia S, Garattini S. Pharmacokinetic and pharmacodynamic significance of antidepressant drug metabolites. Pharmacol Res 1992; 26: 317-329

[174] Caccia S, Invernizzi RW, Nobili A et al. A new generation of antipsychotics: pharmacology and clinical utility of cariprazine in schizophrenia. Ther Clin Risk Manag 2013; 9: 319-328

[175] Caccia S, Pasina L, Nobili A. New atypical antipsychotics for schizophrenia: iloperidone. Drug Des Devel Ther 2010; 4: 33-48

[176] Callaghan JT, Bergstrom RF, Ptak LR et al. Olanzapine. Pharmacokinetic and pharmacodynamic profile. Clin Pharmacokinet 1999; 37: 177-193

[177] Canal-Raffin M, Deridet E, Titier K et al. Simplified ultraviolet liquid chromatographic method for determination of sertindole, dehydrosertindole and norsertindole, in human plasma. J Chromatogr B Analyt Technol Biomed Life Sci 2005; 814: 61-67

[178] Canal-Raffin M, Titier K, Deridet E et al. Myocardium distribution of sertindole and its metabolite dehydrosertindole in guinea-pigs. Biopharm Drug Dispos 2006; 27: 171-179

[179] Capone D, Tarantino G, Gentile A et al. Effects of voriconazole on tacrolimus metabolism in a kidney transplant recipient. J Clin Pharm Ther 2010; 35: 121-124

[180] Carlsson B, Olsson G, Reis M et al. Enantioselective analysis of citalopram and metabolites in adolescents. Ther Drug Monit 2001; 23: 658-664

[181] Carnovale C, Pellegrino P, Beretta S et al. A case of tinnitus induced by chlorpromazine in a pediatric patient. J Pharmacol Pharmacother 2014; 5: 163-165

[182] Carrillo JA, Benitez J. Clinically significant pharmacokinetic interactions between dietary caffeine and medications. Clin Pharmacokinet 2000; 39: 127-153

[183] Castberg I, Skogvoll E, Spigset O. Quetiapine and drug interactions: evidence from a routine therapeutic drug monitoring service. J Clin Psychiatry 2007; 68: 1540-1545

[184] Castberg I, Spigset O. Prescribing patterns and the use of therapeutic drug monitoring of psychotropic medication in a psychiatric high-security unit. Ther Drug Monit 2008; 30: 597-603 
[185] Causon R. Validation of chromatographic methods in biomedical analysis. Viewpoint and discussion. J Chromatogr B Biomed Sci Appl 1997; 689: 175-180

[186] Cawello W. Clinical pharmacokinetic and pharmacodynamic profile of lacosamide. Clin Pharmacokinet 2015; 54: 901-914

[187] Cawello W, Braun M, Boekens H. Absorption, disposition, metabolic fate, and elimination of the dopamine agonist rotigotine in man: administration by intravenous infusion or transdermal delivery. Drug Metab Dispos 2009; 37: 2055-2060

[188] Cawello W, Fichtner A, Boekens $\mathrm{H}$ et al. Influence of hepatic impairment on the pharmacokinetics of the dopamine agonist rotigotine. Eur J Drug Metab Pharmacokinet 2014; 39: 155-163

[189] Centerholt C, Ekblom M, Odergren T et al. Pharmacokinetics and sedative effects in healthy subjects and subjects with impaired liver function after continuous infusion of clomethiazole. Eur J Clin Pharmacol 2003; 59: 117-122

[190] Ceskova E. The need to improve current psychopharmacotherapy before developing new drugs. Expert Opin Pharmacother 2014; 15: 1969-1973

[191] Chakraborty BS, Midha KK, McKay G et al. Single dose kinetics of thioridazine and its two psychoactive metabolites in healthy humans: A dose proportionality study. J Pharm Sci 1989; 78: 796-801

[192] Chamorro AJ, Marcos M, Miron-Canelo JA et al. Association of micro-opioid receptor (OPRM1) gene polymorphism with response to naltrexone in alcohol dependence: a systematic review and meta-analysis. Addict Biol 2012; 17: 505-512

[193] Chan LN, Anderson GD. Pharmacokinetic and pharmacodynamic drug interactions with ethanol (alcohol). Clin Pharmacokinet 2014; 53: $1115-1136$

[194] Chan V, Morris RG, llett KF et al. Population pharmacokinetics of lamotrigine. Ther Drug Monit 2001; 23: 630-635

[195] Chang M, Tybring G, Dahl ML et al. Impact of cytochrome P450 2C19 polymorphisms on citalopram/escitalopram exposure: A systematic review and meta-analysis. Clin Pharmacokinet 2014; 53: 801-811

[196] Chang YC, Lane HY, Yang KH et al. Optimizing early prediction for antipsychotic response in schizophrenia. J Clin Psychopharmacol 2006; 26: 554-559

[197] Chapel S, Hutmacher MM, Haig G et al. Exposure-response analysis in patients with schizophrenia to assess the effect of asenapine on QTC prolongation. J Clin Pharmacol 2009; 49: 1297-1308

[198] Chappell JC, Eisenhofer G, Owens M] et al. Effects of duloxetine on norepinephrine and serotonin transporter activity in healthy subjects. J Clin Psychopharmacol 2014; 34: 9-16

[199] Chawarski MC, Schottenfeld RS, O'Connor PG et al. Plasma concentrations of buprenorphine 24 to 72 hours after dosing. Drug Alcohol Depend 1999; 55: 157-163

[200] Chen G, Lee R, Hojer AM et al. Pharmacokinetic drug interactions involving vortioxetine (Lu AA21004), a multimodal antidepressant. Clin Drug Investig 2013; 33: 727-736

[201] Chen H, Grover S, Yu L et al. Bioactivation of lamotrigine in vivo in rat and in vitro in human liver microsomes, hepatocytes, and epidermal keratinocytes: characterization of thioether conjugates by liquid chromatography/mass spectrometry and high field nuclear magnetic resonance spectroscopy. Chem Res Toxicol 2010; 23: 159-170

[202] Chen L, Greenberg WM, Brand-Schieber E et al. Effect of renal impairment on the pharmacokinetics of levomilnacipran following a single oral dose of levomilnacipran extended-release capsule in humans. Drug Des Devel Ther 2015; 9: 3293-3300

[203] Chen L, Greenberg WM, Gommoll C et al. Levomilnacipran pharmacokinetics in healthy volunteers versus patients with major depressive disorder and implications for norepinephrine and serotonin reuptake inhibition. Clin Ther 2015; 37: 2059-2070
[204] Chen P, Tanasijevic M], Schoenenberger RA et al. A computer-based intervention for improving the appropriateness of antiepileptic drug level monitoring. Am J Clin Pathol 2003; 119: 432-438

[205] Cheng YF, Paalzow LK, Bondesson U et al. Pharmacokinetics of haloperidol in psychotic patients. Psychopharmacology (Berl) 1987; 91: 410-414

[206] Chenu F, Batten LA, Zernig G et al. Comparison of pharmacokinetic profiles of brand-name and generic formulations of citalopram and venlafaxine: a crossover study. J Clin Psychiatry 2009; 70: 958-966

[207] Cherma MD, Lofgren UB, Almkvist G et al. Assessment of the prescription of antidepressant drugs in elderly nursing home patients: a clinical and laboratory follow-up investigation. J Clin Psychopharmacol 2008; 28: 424-431

[208] Cherma MD, Reis M, Hagg S et al. Therapeutic drug monitoring of ziprasidone in a clinical treatment setting. Ther Drug Monit 2008; 30: 682-688

[209] Cherubini A, Nistico G, Rozzini R et al. Subthreshold depression in older subjects: an unmet therapeutic need. J Nutr Health Aging 2012; 16: 909-913

[210] Chetty M, Gouws E, Miller R et al. The use of a side effect as a qualitative indicator of plasma chlorpromazine levels. Eur Neuropsychopharmacol 1999; 9: 77-82

[211] Chew ML, Alvey CW, Plotka A et al. Pregabalin controlled-release pharmacokinetics in healthy volunteers: Analysis of four multipledose randomized clinical pharmacology studies. Clin Drug Investig 2014; 34: 627-637

[212] Chew ML, Mulsant BH, Pollock BG et al. Anticholinergic activity of 107 medications commonly used by older adults. J Am Geriatr Soc 2008; 56: 1333-1341

[213] Chiu YY, Ereshefsky L, Preskorn SH et al. Lurasidone drug-drug interaction studies: A comprehensive review. Drug Metabol Drug Interact 2014; 29: 191-202

[214] Cho HY, Yoo HD, Lee YB. Influence of ABCB1 genetic polymorphisms on the pharmacokinetics of levosulpiride in healthy subjects. Neuroscience 2010; 169: 378-387

[215] Cho JY, Yu KS, Jang IJ et al. Omeprazole hydroxylation is inhibited by a single dose of moclobemide in homozygotic EM genotype for CYP2C19. Br J Clin Pharmacol 2002; 53: 393-397

[216] Choc MG, Hsuan F, Honigfeld G et al. Single- vs multiple-dose pharmacokinetics of clozapine in psychiatric patients. Pharm Res 1990; 7: 347-351

[217] Choi Cl, Bae JW, Lee Y] et al. Effects of CYP2C19 genetic polymorphisms on atomoxetine pharmacokinetics. J Clin Psychopharmacol 2014; 34: 139-142

[218] Christensen M, Andersson K, Dalen P et al. The Karolinska cocktail for phenotyping of five human cytochrome P450 enzymes. Clin Pharmacol Ther 2003; 73: 517-528

[219] Chu XM, Zhang LF, Wang G] et al. Influence of UDP-glucuronosyltransferase polymorphisms on valproic acid pharmacokinetics in Chinese epilepsy patients. Eur J Clin Pharmacol 2012; 68: 1395-1401

[220] Ciraulo DA, Hitzemann RJ, Somoza E et al. Pharmacokinetics and pharmacodynamics of multiple sublingual buprenorphine tablets in dose-escalation trials. J Clin Pharmacol 2006; 46: 179-192

[221] Ciszowski K, Szpak D, Wilimowska J et al. Sulpiride poisoning--case report confirmed with the quantitative determination of the xenobiotic serum level. Przegl Lek 2011; 68: 506-509

[222] Citrome L. Asenapine review, part I: Chemistry, receptor affinity profile, pharmacokinetics and metabolism. Expert Opin Drug Metab Toxicol 2014; 10: 893-903

[223] Citrome L. Brexpiprazole: A new dopamine $D(2)$ receptor partial agonist for the treatment of schizophrenia and major depressive disorder. Drugs Today (Barc). 2015: 51: 397-414 
[224] Citrome L. Paliperidone palmitate - review of the efficacy, safety and cost of a new second-generation depot antipsychotic medication. Int J Clin Pract 2010; 64: 216-239

[225] Citrome L. A review of the pharmacology, efficacy and tolerability of recently approved and upcoming oral antipsychotics: An evidencebased medicine approach. CNS Drugs 2013; 27: 879-911

[226] Citrome L, Stauffer VL, Chen L et al. Olanzapine plasma concentrations after treatment with 10,20 , and $40 \mathrm{mg} / \mathrm{d}$ in patients with schizophrenia: An analysis of correlations with efficacy, weight gain, and prolactin concentration. J Clin Psychopharmacol 2009; 29: 278-283

[227] Coghill D, Banaschewski T, Zuddas A et al. Long-acting methylphenidate formulations in the treatment of attention-deficit/hyperactivity disorder: A systematic review of head-to-head studies. BMC Psychiatry 2013; 13: 237

[228] Coller JK, Somogyi AA, Bochner F. Flunitrazepam oxidative metabolism in human liver microsomes: Involvement of CYP2C19 and CYP3A4. Xenobiotica 1999; 29: 973-986

[229] Collins FS, Varmus H. A new initiative on precision medicine. N Engl ] Med 2015; 372: 793-795

[230] Collins N, Barnes TR, Shingleton-Smith A et al. Standards of lithium monitoring in mental health trusts in the UK. BMC Psychiatry 2010; 10: 80

[231] Conca A, Schmidt E, Pastore M et al. Therapeutic drug monitoring in Italian psychiatry. Pharmacopsychiatry 2011; 44: 259-262

[232] Connarn JN, Zhang X, Babiskin A et al. Metabolism of bupropion by carbonyl reductases in liver and intestine. Drug Metab Dispos 2015; 43: 1019-1027

[233] Connolly KR, Thase ME. The clinical management of bipolar disorder: a review of evidence-based guidelines. Prim Care Companion CNS Disord 2011; 13: pii: PCC.10r01097

[234] Contin M, Albani F, Riva R et al. Lacosamide therapeutic monitoring in patients with epilepsy: effect of concomitant antiepileptic drugs. Ther Drug Monit 2013; 35: 849-852

[235] Contin M, Mohamed S, Albani F et al. Levetiracetam clinical pharmacokinetics in elderly and very elderly patients with epilepsy. Epilepsy Res 2012; 98: 130-134

[236] Contin M, Riva R, Martinelli P et al. Effect of meal timing on the kinetic-dynamic profile of levodopa/carbidopa controlled release [corrected] in parkinsonian patients. Eur J Clin Pharmacol 1998; 54 : 303-308

[237] Cooper TB. Plasma level monitoring of antipsychotic drugs. Clin Pharmacokinet 1978; 3: 14-38

[238] Coppen A, Kopera H. Workshop on the clinical pharmacology and efficacy of mianserin. Br J Clin Pharmacol 1978; 5: (Suppl 1): 91S-99S

[239] Correll CU, Malhotra AK, Kaushik S et al. Early prediction of antipsychotic response in schizophrenia. Am J Psychiatry 2003; 160: 2063-2065

[240] Couchman L, Bowskill SV, Handley S et al. Plasma clozapine and norclozapine in relation to prescribed dose and other factors in patients aged $<18$ years: Data from a therapeutic drug monitoring service, 1994-2010. Early Interv Psychiatry 2013; 7: 122-130

[241] Couchman L, Morgan PE, Spencer EP et al. Plasma clozapine, norclozapine, and the clozapine: Norclozapine ratio in relation to prescribed dose and other factors: data from a therapeutic drug monitoring service, 1993-2007. Ther Drug Monit 2010; 32: 438-447

[242] Couchman L, Morgan PE, Spencer EP et al. Plasma clozapine and norclozapine in patients prescribed different brands of clozapine (Clozaril, Denzapine, and Zaponex). Ther Drug Monit 2010; 32: 624-627

[243] Coulomb F, Ducret F, Laneury JP et al. Pharmacokinetics of singledose reboxetine in volunteers with renal insufficiency. | Clin Pharmacol 2000; 40: 482-487
[244] Cournoyer G, de Montigny C, Ouellette J et al. A comparative double-blind controlled study of trimipramine and amitriptyline in major depression: Lack of correlation with 5-hydroxytryptamine reuptake blockade. J Clin Psychopharmacol 1987; 7: 385-393

[245] Court MH. Interindividual variability in hepatic drug glucuronidation: studies into the role of age, sex, enzyme inducers, and genetic polymorphism using the human liver bank as a model system. Drug Metab Rev 2010; 42: 209-224

[246] Court MH, Duan SX, Guillemette C et al. Stereoselective conjugation of oxazepam by human UDP-glucuronosyltransferases (UGTs): $\mathrm{S}$-oxazepam is glucuronidated by UGT2B15, while R-oxazepam is glucuronidated by UGT2B7 and UGT1A9. Drug Metab Dispos 2002; 30: 1257-1265

[247] Crettol S, Besson J, Croquette-Krokar M et al. Association of dopamine and opioid receptor genetic polymorphisms with response to methadone maintenance treatment. Prog Neuropsychopharmacol Biol Psychiatry 2008; 32: 1722-1727

[248] Crettol S, de Leon J, Hiemke C et al. Pharmacogenomics in psychiatry: From therapeutic drug monitoring to genomic medicine. Clin Pharmacol Ther 2014; 95: 254-257

[249] Crettol S, Deglon JJ, Besson J et al. ABCB1 and cytochrome P450 genotypes and phenotypes: Influence on methadone plasma levels and response to treatment. Clin Pharmacol Ther 2006; 80: 668-681

[250] Crettol S, Deglon J], Besson J et al. Methadone enantiomer plasma levels, CYP2B6, CYP2C19, and CYP2C9 genotypes, and response to treatment. Clin Pharmacol Ther 2005; 78: 593-604

[251] Csajka C, Crettol S, Guidi M et al. Population Genetic-Based Pharmacokinetic Modeling of Methadone and its Relationship with the QTc Interval in Opioid-Dependent Patients. Clin Pharmacokinet 2016; 55: 1521-1533

[252] Cummings ], Lefevre G, Small G et al. Pharmacokinetic rationale for the rivastigmine patch. Neurology 2007; 69: S10-S13

[253] Cutler NR, Polinsky R], Sramek J] et al. Dose-dependent CSF acetylcholinesterase inhibition by SDZ ENA 713 in Alzheimer's disease. Acta Neurol Scand 1998; 97: 244-250

[254] Dahl SG. Active metabolites of neuroleptic drugs: possible contribution to therapeutic and toxic effects. Ther Drug Monit 1982; 4: 33-40

[255] Dahl SG, Strandjord RE, Sigfusson S. Pharmacokinetics and relative bioavailability of levomepromazine after repeated administration of tablets and syrup. Eur J Clin Pharmacol 1977; 11: 305-310

[256] Damoiseaux VA, Proost JH, Jiawan VC et al. Sex differences in the pharmacokinetics of antidepressants: Influence of female sex hormones and oral contraceptives. Clin Pharmacokinet 2014; 53: 509-519

[257] Darby JK, Pasta DJ, Wilson MG et al. Long-term therapeutic drug monitoring of risperidone and olanzapine identifies altered steady-state pharmacokinetics: A clinical, two-group, naturalistic study. Clin Drug Investig 2008; 28: 553-564

[258] Davies SJ, Westin AA, Castberg I et al. Characterisation of zuclopenthixol metabolism by in vitro and therapeutic drug monitoring studies. Acta Psychiatr Scand 2010; 122: 444-453

[259] Daviss WB, Perel JM, Birmaher B et al. Steady-state clinical pharmacokinetics of bupropion extended-release in youths. J Am Acad Child Adolesc Psychiatry 2006; 45: 1503-1509

[260] Daviss WB, Perel JM, Brent DA et al. Acute antidepressant response and plasma levels of bupropion and metabolites in a pediatric-aged sample: an exploratory study. Ther Drug Monit 2006; 28: 190-198

[261] Dawling S. Monitoring of tricyclic antidepressant therapy. Clin Biochem 1982; 15: 56-61

[262] De Gregori S, De Gregori M, Ranzani GN et al. Morphine metabolism, transport and brain disposition. Metab Brain Dis 2012; 27: 1-5

[263] de Klerk OL, Nolte IM, Bet PM et al. ABCB1 gene variants influence tolerance to selective serotonin reuptake inhibitors in a large sample 
of Dutch cases with major depressive disorder. Pharmacogenomics ] 2013; 13: 349-353

[264] De las Cuevas C, Penate W, Sanz EJ. Risk factors for non-adherence to antidepressant treatment in patients with mood disorders. Eur J Clin Pharmacol 2014; 70: 89-98

[265] de Leon J. The crucial role of the therapeutic window in understanding the clinical relevance of the poor versus the ultrarapid metabolizer phenotypes in subjects taking drugs metabolized by CYP2D6 or CYP2C19. J Clin Psychopharmacol 2007; 27: 241-245

[266] de Leon J. The effects of antiepileptic inducers in neuropsychopharmacology, a neglected issue. Part I: A summary of the current state for clinicians. Rev Psiquiatr Salud Ment 2015; 8: 97-115

[267] de J. Focusing on drug versus disease mechanisms and on clinical subgrouping to advance personalised medicine in psychiatry. Acta Neuropsychiatr 2014; 26: 327-333

[268] de Leon J. Glucuronidation enzymes, genes and psychiatry. Int ] Neuropsychopharmacol 2003; 6: 57-72

[269] de Leon J. Incorporating pharmacogenetics into clinical practice: reality of a new tool in psychiatry. Current issues in clinical implementation. CNS Spectr 2006; 11: 8-12

[270] de Leon J, Greenlee B, Barber J et al. Practical guidelines for the use of new generation antipsychotic drugs (except clozapine) in adult individuals with intellectual disabilities. Res Dev Disabil 2009; 30 : 613-669

[271] de Leon J, Spina E, Diaz FJ. Clobazam therapeutic drug monitoring: A comprehensive review of the literature with proposals to improve future studies. Ther Drug Monit 2013; 35: 30-47

[272] de Leon J, Susce MT, Pan RM et al. The CYP2D6 poor metabolizer phenotype may be associated with risperidone adverse drug reactions and discontinuation. J Clin Psychiatry 2005; 66: 15-27

[273] de Leon J, Wynn G, Sandson NB. The pharmacokinetics of paliperidone versus risperidone. Psychosomatics 2010; 51: 80-88

[274] de Mey C, Althaus M, Ezan E et al. Erythromycin increases plasma concentrations of alpha-dihydroergocryptine in humans. Clin Pharmacol Ther 2001; 70: 142-148

[275] de Wit M, Best AM, Epstein SK et al. Lorazepam concentrations, pharmacokinetics and pharmacodynamics in a cohort of mechanically ventilated ICU patients. Int J Clin Pharmacol Ther 2006; 44 : 466-473

[276] Degen J, Wolke E, Seiberling M et al. Comparative study of the pharmacokinetics of amitriptyline oxide and trimipramine after single administration in healthy male probands and patients with renal failure. Med Klin (Munich) 1993; 88: 129-133 171

[277] Degner D, Grohmann R, Kropp S et al. Severe adverse drug reactions of antidepressants: results of the German multicenter drug surveillance program AMSP. Pharmacopsychiatry 2004; 37: (Suppl 1): S39-S45

[278] Del Dotto P, Bonuccelli U. Clinical pharmacokinetics of cabergoline. Clin Pharmacokinet 2003; 42: 633-645

[279] Deleu D, Northway MG, Hanssens Y. Clinical pharmacokinetic and pharmacodynamic properties of drugs used in the treatment of Parkinson's disease. Clin Pharmacokinet 2002; 41: 261-309

[280] Deligiannidis KM. Therapeutic drug monitoring in pregnant and postpartum women: Recommendations for SSRIs, lamotrigine, and lithium. J Clin Psychiatry 2010; 71: 649-650

[281] Delva NJ, Hawken ER. Preventing lithium intoxication. Guide for physicians. Can Fam Physician 2001; 47: 1595-1600

[282] Dempsey DA St, Helen G, Jacob P 3rd et al. Genetic and pharmacokinetic determinants of response to transdermal nicotine in white, black, and Asian nonsmokers. Clin Pharmacol Ther 2013; 94 : 687-694
[283] Derungs A, Donzelli M, Berger B et al. Effects of Cytochrome P450 Inhibition and Induction on the phenotyping metrics of the basel cocktail: A randomized crossover study. Clin Pharmacokinet 2016; 55: 79-91

[284] Desta Z, Kerbusch T, Flockhart DA. Effect of clarithromycin on the pharmacokinetics and pharmacodynamics of pimozide in healthy poor and extensive metabolizers of cytochrome P450 2D6 (CYP2D6). Clin Pharmacol Ther 1999; 65: 10-20

[285] Desta Z, Kerbusch T, Soukhova N et al. Identification and characterization of human cytochrome P450 isoforms interacting with pimozide. J Pharmacol Exp Ther 1998; 285: 428-437

[286] Deuschle M, Härtter S, Hiemke C et al. Doxepin and its metabolites in plasma and cerebrospinal fluid in depressed patients. Psychopharmacology (Berl) 1997; 131: 19-22

[287] DeVane CL, Boulton DW, Miller LF et al. Pharmacokinetics of trazodone and its major metabolite m-chlorophenylpiperazine $\mathrm{i}$ n plasma and brain of rats. Int J Neuropsychopharmacol 1999; 2: $17-23$

[288] DeVane CL, Liston HL, Markowitz JS. Clinical pharmacokinetics of sertraline. Clin Pharmacokinet 2002; 41: 1247-1266

[289] DeVane CL, Stowe ZN, Donovan JL et al. Therapeutic drug monitoring of psychoactive drugs during pregnancy in the genomic era: challenges and opportunities. J Psychopharmacol 2006; 20: 54-59

[290] Diaz F], de Leon J, Josiassen RC et al. Plasma clozapine concentration coefficients of variation in a long-term study. Schizophr Res 2005; 72: 131-135

[291] Diaz FJ, Eap CB, Ansermot $\mathrm{N}$ et al. Can valproic acid be an inducer of clozapine metabolism? Pharmacopsychiatry 2014; 47: 89-96

[292] Diaz FJ, Santoro V, Spina E et al. Estimating the size of the effects of co-medications on plasma clozapine concentrations using a model that controls for clozapine doses and confounding variables. Pharmacopsychiatry 2008; 41: 81-91

[293] Dilmaghanian S, Gerber JG, Filler SG et al. Enantioselectivity of inhibition of cytochrome P450 3A4 (CYP3A4) by ketoconazole: Testosterone and methadone as substrates. Chirality 2004; 16: 79-85

[294] Dingemanse J, Jorga K, Zurcher G et al. Multiple-dose clinical pharmacology of the catechol-O-methyl-transferase inhibitor tolcapone in elderly subjects. Eur J Clin Pharmacol 1996; 50: 47-55

[295] Dingemanse J, Wood N, Guentert T et al. Clinical pharmacology of moclobemide during chronic administration of high doses to healthy subjects. Psychopharmacology (Berl) 1998; 140: 164-172

[296] Dinger ], Meyer MR, Maurer HH. Development of an in vitro cytochrome P450 cocktail inhibition assay for assessing the inhibition risk of drugs of abuse. Toxicol Lett 2014; 230: 28-35

[297] Dixon R, Gentile J, Hsu HB et al. Nalmefene: Safety and kinetics after single and multiple oral doses of a new opioid antagonist. J Clin Pharmacol 1987; 27: 233-239

[298] Dockens RC, Salazar DE, Fulmor IE et al. Pharmacokinetics of a newly identified active metabolite of buspirone after administration of buspirone over its therapeutic dose range. J Clin Pharmacol 2006; 46: 1308-1312

[299] Dockens RC, Tran AQ, Zeng J et al. Pharmacokinetics of 6-hydroxybuspirone and its enantiomers administered individually or following buspirone administration in humans. Biopharm Drug Dispos 2007; 28: 393-402

[300] Domschke K, Tidow N, Schwarte K et al. Serotonin transporter gene hypomethylation predicts impaired antidepressant treatment response. Int J Neuropsychopharmacol 2014; 17: 1167-1176

[301] Dong C, Wong ML, Licinio J. Sequence variations of ABCB1, SLC6A2, SLC6A3, SLC6A4, CREB1, CRHR1 and NTRK2: Association with major depression and antidepressant response in Mexican-Americans. Mol Psychiatry 2009; 14: 1105-1118 
[302] Doose DR, Brodie M], Wilson EA et al. Topiramate and lamotrigine pharmacokinetics during repetitive monotherapy and combination therapy in epilepsy patients. Epilepsia 2003; 44: 917-922

[303] Doran A, Obach RS, Smith B] et al. The impact of P-glycoprotein on the disposition of drugs targeted for indications of the central nervous system: Evaluation using the MDR1A/1B knockout mouse model. Drug Metab Dispos 2005; 33: 165-174

[304] Dormann H, Sonst A, Müller F et al. Adverse drug events in older patients admitted as an emergency: The role of potentially inappropriate medication in elderly people (PRISCUS). Dtsch Arztebl Int 2013; 110: 213-219

[305] dos Santos FM, Goncalves JC, Caminha R et al. Pharmacokinetic/ pharmacodynamic modeling of psychomotor impairment induced by oral clonazepam in healthy volunteers. Ther Drug Monit 2009; 31: 566-574

[306] Dost FH. Der Blutspiegel- Kinetik der Konzentrationsabläufe in der Kreislaufkörperflüssigkeit. Leipzig: Georg Thieme; 1953

[307] Dresse A, Rosen JM, Brems H et al. Influence of food on tianeptine and its main metabolite kinetics. J Clin Pharmacol 1988; 28 : $1115-1119$

[308] Drouet-Coassolo C, Iliadis A, Coassolo P et al. Pharmacokinetics of flunitrazepam following single dose oral administration in liver disease patients compared with healthy volunteers. Fundam Clin Pharmacol 1990; 4: 643-651

[309] Drover D, Lemmens H, Naidu S et al. Pharmacokinetics, pharmacodynamics, and relative pharmacokinetic/pharmacodynamic profiles of zaleplon and zolpidem. Clin Ther 2000; 22: 1443-1461

[310] Dubois N, Demaret I, Ansseau M et al. Plasma level monitoring of the major metabolites of diacetylmorphine (heroin) by the "chasing the dragon" route in severe heroin addicts. Acta Clin Belg 2013; 68: 359-367

[311] Dubois N, Hallet C, Seidel L et al. Estimation of the Time Interval between the Administration of Heroin and the Sampling of Blood in Chronic Inhalers. J Anal Toxicol 2015; 39: 300-305

[312] Dubovsky SL, Frobose C, Phiri P et al. Short-term safety and pharmacokinetic profile of asenapine in older patients with psychosis. Int J Geriatr Psychiatry 2012; 27: 472-482

[313] Dunbar JL, Turncliff RZ, Hayes SC et al. Population pharmacokinetics of extended-release injectable naltrexone (XR-NTX) in patients with alcohol dependence. J Stud Alcohol Drugs 2007; 68: 862-870

[314] Dvorchik BH, Vesell ES. Pharmacokinetic interpretation of data gathered during therapeutic drug monitoring. Clin Chem 1976; 22 : 868-878

[315] Dwivedi R, Gupta YK, Singh M et al. Correlation of saliva and serum free valproic acid concentrations in persons with epilepsy. Seizure 2015; 25: 187-190

[316] Dwivedi R, Singh M, Kaleekal T et al. Concentration of antiepileptic drugs in persons with epilepsy: A comparative study in serum and saliva. Int J Neurosci 2016; 126: 972-978

[317] Eagles JM, McCann I, MacLeod TN et al. Lithium monitoring before and after the distribution of clinical practice guidelines. Acta Psychiatr Scand 2000; 101: 349-353

[318] Eap CB, Baumann P. Analytical methods for the quantitative determination of selective serotonin reuptake inhibitors for therapeutic drug monitoring purposes in patients. J Chromatogr B Biomed Appl 1996; 686: 51-63

[319] Eap CB, Bender S, Gastpar M et al. Steady state plasma levels of the enantiomers of trimipramine and of its metabolites in CYP2D6-, CYP2C19- and CYP3A4/5-phenotyped patients. Ther Drug Monit 2000; 22: 209-214

[320] Eap CB, Bertschy G, Baumann P et al. High interindividual variability of methadone enantiomer blood levels to dose ratios. Arch Gen Psychiatry 1998; 55: 89-90
[321] Eap CB, Bourquin M, Martin J et al. Plasma concentrations of the enantiomers of methadone and therapeutic response in methadone maintenance treatment. Drug Alcohol Depend 2000; 61: 47-54

[322] Eap CB, Buclin T, Baumann P. Interindividual variability of the clinical pharmacokinetics of methadone: Implications for the treatment of opioid dependence. Clin Pharmacokinet 2002; 41: 1153-1193

[323] Eap CB, Finkbeiner T, Gastpar M et al. Replacement of (R)-methadone by a double dose of $(\mathrm{R}, \mathrm{S})$-methadone in addicts: Interindividual variability of the $(R) /(S)$ ratios and evidence of adaptive changes in methadone pharmacokinetics. Eur J Clin Pharmacol 1996; 50: 385-389

[324] Eap CB, Guentert TW, Schaublin-Loidl M et al. Plasma levels of the enantiomers of thioridazine, thioridazine 2-sulfoxide, thioridazine 2-sulfone, and thioridazine 5 -sulfoxide in poor and extensive metabolizers of dextromethorphan and mephenytoin. Clin Pharmacol Ther 1996; 59: 322-331

[325] Eap CB, Koeb L, Baumann P. Determination of trimipramine and its demethylated and hydroxylated metabolites in plasma by gas chromatography-mass spectrometry. J Chromatogr 1994; 652: 97-103

[326] Eap CB, Lima CA, Macciardi F et al. Steady state concentrations of the enantiomers of mianserin and desmethylmianserin in poor and in homozygous and heterozygous extensive metabolizers of debrisoquine. Ther Drug Monit 1998; 20: 7-13

[327] Egberts K, Karwautz A, Plener PL et al. Pharmacovigilance in child and adolescent psychiatry. Z Kinder Jugendpsychiatr Psychother 2015; 43: 21-28

[328] Egberts KM, Mehler-Wex C, Gerlach M. Therapeutic drug monitoring in child and adolescent psychiatry. Pharmacopsychiatry 2011; 44: 249-253

[329] Eggart V, Hiemke C, Zernig G. "There is no dose-response relationship in psychopharmacotherapy" vs "pharmacotherapy in psychiatry is based on ligand-receptor interaction": A unifying hypothesis and the need for plasma concentration based clinical trials. Psychopharmacology (Berl) 2011; 217: 297-300

[330] Eggert Hansen C, Rosted Christensen T, Elley J et al. Clinical pharmacokinetic studies of perphenazine. Br J Clin Pharmacol 1976; 3: 915-923

[331] Ehmann F, Caneva L, Prasad K et al. Pharmacogenomic information in drug labels: European medicines agency perspective. Pharmacogenomics J 2015; 15: 201-210

[332] Eichelbaum M, Ingelman-Sundberg M, Evans WE. Pharmacogenomics and individualized drug therapy. Annu Rev Med 2006; 57: 119-137

[333] El Desoky ES, Sabarinath SN, Hamdi MM et al. Population pharmacokinetics of steady-state carbamazepine in Egyptian epilepsy patients. J Clin Pharm Ther 2012; 37: 352-355

[334] Ellinwood EH Jr., Heatherly DG, Nikaido AM et al. Comparative pharmacokinetics and pharmacodynamics of lorazepam, alprazolam and diazepam. Psychopharmacology (Berl) 1985; 86: 392-399

[335] Elshoff JP, Cawello W, Andreas JO et al. An update on pharmacological, pharmacokinetic properties and drug-drug interactions of rotigotine transdermal system in Parkinson's disease and restless legs syndrome. Drugs 2015; 75: 487-501

[336] Ereshefsky L, Jann MW, Saklad SR et al. Effects of smoking on fluphenazine clearance in psychiatric inpatients. Biol Psychiatry 1985; 20: $329-332$

[337] Erickson-Ridout KK, Zhu J, Lazarus P. Olanzapine metabolism and the significance of UGT1A448V and UGT2B1067Y variants. Pharmacogenet Genomics 2011; 21: 539-551

[338] Ermer J, Corcoran M, Lasseter K et al. Relative bioavailabilities of lisdexamfetamine dimesylate and $\mathrm{d}$-amphetamine in healthy adults in an open-label, randomized, crossover study after mixing lisdexamfetamine dimesylate with food or drink. Ther Drug Monit 2016; 38: 769-776 
[339] Ermer ], Haffey MB, Richards C et al. An open-label investigation of the pharmacokinetic profiles of lisdexamfetamine dimesylate and venlafaxine extended-release, administered alone and in combination, in healthy adults. Clin Drug Investig 2013; 33: 243-254

[340] Evans LE, Bett JH, Cox JR et al. The bioavailability of oral and parenteral chlorimipramine (Anafranil). Prog Neuropsychopharmacol 1980; 4: 293-302

[341] Evans WE, Relling MV. Pharmacogenomics: Translating functional genomics into rational therapeutics. Science 1999; 286: 487-491

[342] Faber MS, Fuhr U. Time response of cytochrome P450 1A2 activity on cessation of heavy smoking. Clin Pharmacol Ther 2004; 76: 178-184

[343] Faber MS, Jetter A, Fuhr U. Assessment of CYP1A2 activity in clinical practice: Why, how, and when? Basic Clin Pharmacol Toxicol 2005; 97: 125-134

[344] Faessel HM, Gibbs MA, Clark DJ et al. Multiple-dose pharmacokinetics of the selective nicotinic receptor partial agonist, varenicline, in healthy smokers. J Clin Pharmacol 2006; 46: 1439-1448

[345] Faiman MD, Jensen JC, Lacoursiere RB. Elimination kinetics of disulfiram in alcoholics after single and repeated doses. Clin Pharmacol Ther 1984; 36: 520-526

[346] Fanton L, Bevalot F, Grait $\mathrm{H}$ et al. Fatal intoxication with milnacipran. J Forensic Leg Med 2008; 15: 388-390

[347] Farde L, Nordstrom AL, Wiesel FA et al. Positron emission tomographic analysis of central D1 and D2 dopamine receptor occupancy in patients treated with classical neuroleptics and clozapine. Relation to extrapyramidal side effects. Arch Gen Psychiatry 1992; 49 : 538-544

[348] Fasano A, Bove F, Gabrielli M et al. Liquid melevodopa versus standard levodopa in patients with Parkinson disease and small intestinal bacterial overgrowth. Clin Neuropharmacol 2014; 37: 91-95

[349] Fellows L, Ahmad F, Castle D] et al. Investigation of target plasma concentration-effect relationships for olanzapine in schizophrenia. Ther Drug Monit 2003; 25: 682-689

[350] Feng Y, Pollock BG, Coley K et al. Population pharmacokinetic analysis for risperidone using highly sparse sampling measurements from the CATIE study. Br J Clin Pharmacol 2008; 66: 629-639

[351] Feng Y, Pollock BG, Ferrell RE et al. Paroxetine: Population pharmacokinetic analysis in late-life depression using sparse concentration sampling. Br J Clin Pharmacol 2006; 61: 558-569

[352] Fernandez C, Martin C, Gimenez F et al. Clinical pharmacokinetics of zopiclone. Clin Pharmacokinet 1995; 29: 431-441

[353] Ferrari A, Bertolotti M, Dell'Utri A et al. Serum time course of naltrexone and 6 beta-naltrexol levels during long-term treatment in drug addicts. Drug Alcohol Depend 1998; 52: 211-220

[354] Ferrell PB Jr., McLeod HL. Carbamazepine, HLA-B * 1502 and risk of Stevens-Johnson syndrome and toxic epidermal necrolysis: US FDA recommendations. Pharmacogenomics 2008; 9: 1543-1546

[355] Ferron GM, Paul J, Fruncillo R et al. Multiple-dose, linear, dose-proportional pharmacokinetics of retigabine in healthy volunteers. J Clin Pharmacol 2002; 42: 175-182

[356] Figueroa C, Brecher M, Hamer-Maansson JE et al. Pharmacokinetic profiles of extended release quetiapine fumarate compared with quetiapine immediate release. Prog Neuropsychopharmacol Biol Psychiatry 2009; 33: 199-204

[357] Filser JG, Kaumeier S, Brand T et al. Pharmacokinetics of amitriptyline and amitriptylinoxide after intravenous or oral administration in humans. Pharmacopsychiatry 1988; 21: 381-383

[358] Findling RL, Goldman R, Chiu YY et al. Pharmacokinetics and Tolerability of Lurasidone in Children and Adolescents With Psychiatric Disorders. Clin Ther 2015; 37: 2788-2797
[359] Findling RL, Nucci G, Piergies AA et al. Multiple dose pharmacokinetics of paroxetine in children and adolescents with major depressive disorder or obsessive-compulsive disorder. Neuropsychopharmacology 2006; 31: 1274-1285

[360] Fischer M, Unterecker S, Deckert J et al. Elevated clozapine serum levels in combination with perazine. Psychopharmacology (Berl) 2013; 226: 623-625

[361] Fisher DS, Handley SA, Flanagan RJ et al. Plasma concentrations of quetiapine, $\mathrm{N}$-desalkylquetiapine, o-desalkylquetiapine, 7-hydroxyquetiapine, and quetiapine sulfoxide in relation to quetiapine dose, formulation, and other factors. Ther Drug Monit 2012; 34: 415-421

[362] Fisher DS, van Schalkwyk GI, Seedat S et al. Plasma, oral fluid, and whole-blood distribution of antipsychotics and metabolites in clinical samples. Ther Drug Monit 2013; 35: 345-351

[363] Fitzgerald PB, Kapur S, Remington G et al. Predicting haloperidol occupancy of central dopamine D2 receptors from plasma levels. Psychopharmacology (Berl) 2000; 149: 1-5

[364] Flanagan RJ. Developing an analytical toxicology service: principles and guidance. Toxicol Rev 2004; 23: 251-263

[365] Fleischhacker WW, Sanchez R, Perry PP et al. Aripiprazole oncemonthly for treatment of schizophrenia: Double-blind, randomised, non-inferiority study. Br J Psychiatry 2014; 205: 135-144

[366] Fleming J, Chetty M. Therapeutic monitoring of valproate in psychiatry: how far have we progressed? Clin Neuropharmacol 2006; 29: $350-360$

[367] Fogelman SM, Schmider J, Venkatakrishnan K et al. O- and N-demethylation of venlafaxine in vitro by human liver microsomes and by microsomes from cDNA-transfected cells: Effect of metabolic inhibitors and SSRI antidepressants. Neuropsychopharmacology 1999; 20: 480-490

[368] Foisy MM, Yakiwchuk EM, Hughes CA. Induction effects of ritonavir: implications for drug interactions. Ann Pharmacother 2008; 42: 1048-1059

[369] Fontaine R, Mercier P, Beaudry P et al. Bromazepam and lorazepam in generalized anxiety: a placebo-controlled study with measurement of drug plasma concentrations. Acta Psychiatr Scand 1986; 74: 451-458

[370] Food and Drug Administration. Guidance for industry: bioanalytical method validation. 2001; http://www.fda.gov/cvm

[371] Foster D], Somogyi AA, Dyer KR et al. Steady-state pharmacokinetics of (R)- and (S)-methadone in methadone maintenance patients. $\mathrm{Br}$ J Clin Pharmacol 2000; 50: 427-440

[372] Foti RS, Rock DA, Wienkers LC et al. Selection of alternative CYP3A4 probe substrates for clinical drug interaction studies using in vitro data and in vivo simulation. Drug Metab Dispos 2010; 38: 981-987

[373] Frank D, Jaehde U, Fuhr U. Evaluation of probe drugs and pharmacokinetic metrics for CYP2D6 phenotyping. Eur J Clin Pharmacol 2007; 63: 321-333

[374] Freeman MP, Nolan PE Jr., Davis MF et al. Pharmacokinetics of sertraline across pregnancy and postpartum. J Clin Psychopharmacol 2008; 28: 646-653

[375] Fric M, Pfuhlmann B, Laux G et al. The influence of smoking on the serum level of duloxetine. Pharmacopsychiatry 2008; 41: 151-155

[376] Friden M, Winiwarter S, Jerndal G et al. Structure-brain exposure relationships in rat and human using a novel data set of unbound drug concentrations in brain interstitial and cerebrospinal fluids. J Med Chem 2009; 52: 6233-6243

[377] Frieboes RM, Sonntag A, Yassouridis A et al. Clinical outcome after trimipramine in patients with delusional depression - a pilot study. Pharmacopsychiatry 2003; 36: 12-17

[378] Friedman H, Greenblatt DJ, Peters GR et al. Pharmacokinetics and pharmacodynamics of oral diazepam: Effect of dose, plasma concentration, and time. Clin Pharmacol Ther 1992; 52: 139-150 
[379] Friedrich ME, Akimova E, Huf W et al. Drug-induced liver injury during antidepressant treatment: Results of AMSP, a drug surveillance program. Int J Neuropsychopharmacol 2016; 19:

[380] Fritze J, Laux G, Sofic E et al. Plasma moclobemide and metabolites: Lack of correlation with clinical response and biogenic amines. Psychopharmacology (Berl) 1989; 99: 252-256

[381] Froemming JS, Lam YW, Jann MW et al. Pharmacokinetics of haloperidol. Clin Pharmacokinet 1989; 17: 396-423

[382] Froscher W, Schier KR, Hoffmann M et al. Topiramate: A prospective study on the relationship between concentration, dosage and adverse events in epileptic patients on combination therapy. Epileptic Disord 2005; 7: 237-248

[383] Frye RF, Matzke GR, Jallad NS et al. The effect of age on the pharmacokinetics of the opioid antagonist nalmefene. $\mathrm{Br}$ J Clin Pharmacol 1996; 42: 301-306

[384] Fudio S, Borobia AM, Pinana E et al. Evaluation of the influence of sex and CYP2C19 and CYP2D6 polymorphisms in the disposition of citalopram. Eur J Pharmacol 2010; 626: 200-204

[385] Fuhr U, Anders EM, Mahr G et al. Inhibitory potency of quinolone antibacterial agents against cytochrome P450IA2 activity in vivo and in vitro. Antimicrob Agents Chemother 1992; 36: 942-948

[386] Fuhr U, Rost KL, Engelhardt R et al. Evaluation of caffeine as a test drug for CYP1A2, NAT2 and CYP2E1 phenotyping in man by in vivo versus in vitro correlations. Pharmacogenetics 1996; 6: 159-176

[387] Fukasawa T, Suzuki A, Otani K. Effects of genetic polymorphism of cytochrome P450 enzymes on the pharmacokinetics of benzodiazepines. J Clin Pharm Ther 2007; 32: 333-341

[388] Furukori $\mathrm{H}$, Kondo T, Yasui $\mathrm{N}$ et al. Effects of itraconazole on the steady-state plasma concentrations of bromperidol and reduced bromperidol in schizophrenic patients. Psychopharmacology (Berl) 1999; 145: 189-192

[389] Gaedigk A, Simon SD, Pearce RE et al. The CYP2D6 activity score: translating genotype information into a qualitative measure of phenotype. Clin Pharmacol Ther 2008; 83: 234-242

[390] Gaertner H], Golfinopoulos G, Breyer-Pfaff U. Response to maprotiline treatment in depressive patients relationship to urinary MHPG excretion and plasma drug level. Pharmacopsychiatria 1982; 15: 170-174

[391] Gaertner I, Gaertner HJ, Vonthein R et al. Therapeutic drug monitoring of clozapine in relapse prevention: A five-year prospective study. J Clin Psychopharmacol 2001; 21: 305-310

[392] Gafni I, Busto UE, Tyndale RF et al. The role of cytochrome P450 2C19 activity in flunitrazepam metabolism in vivo. J Clin Psychopharmacol 2003; 23: 169-175

[393] Gallagher P, O’Mahony D. STOPP (Screening Tool of Older Persons' potentially inappropriate Prescriptions): application to acutely ill elderly patients and comparison with Beers' criteria. Age Ageing 2008; 37: 673-679

[394] Garg V, Chandorkar G, Farmer HF et al. Effect of telaprevir on the pharmacokinetics of midazolam and digoxin. J Clin Pharmacol 2012; 52: $1566-1573$

[395] Garnock-Jones KP, Keating GM. Atomoxetine: A review of its use in attention-deficit hyperactivity disorder in children and adolescents. Paediatr Drugs 2009; 11: 203-226

[396] Gastpar M, Heinz W, Poehlke T et al. Substitutionstherapie bei Drogenabhängigkeit. 2. überarbeitete AuflageBerlin, Heidelberg, New York: Springer Verlag; 2002

[397] Genton P, Guerrini R, Perucca E. Tiagabine in clinical practice. Epilepsia 2001; 42: (Suppl 3): 42-45

[398] Geretsegger C. Psychopharmaka-Spiegel bei Gutachten in Pensionsverfahren. Proc DGPPN 2006; P0169
[399] Gerlach M, Egberts K, Dang SY et al. Therapeutic drug monitoring as a measure of proactive pharmacovigilance in child and adolescent psychiatry. Expert Opin Drug Saf 2016; 15: 1477-1482

[400] Gerlach M, Hunnerkopf R, Rothenhofer S et al. Therapeutic drug monitoring of quetiapine in adolescents with psychotic disorders. Pharmacopsychiatry 2007; 40: 72-76

[401] Gerstenberg G, Aoshima T, Fukasawa T et al. Relationship between clinical effects of fluvoxamine and the steady-state plasma concentrations of fluvoxamine and its major metabolite fluvoxamino acid in Japanese depressed patients. Psychopharmacology (Berl) 2003; 167: 443-448

[402] Gervasini G, Carrillo JA, Benitez J. Potential role of cerebral cytochrome P450 in clinical pharmacokinetics: Modulation by endogenous compounds. Clin Pharmacokinet 2004; 43: 693-706

[403] Gex-Fabry M, Balant-Gorgia AE, Balant LP. Clomipramine concentration as a predictor of delayed response: A naturalistic study. Eur J Clin Pharmacol 1999; 54: 895-902

[404] Gex-Fabry M, Balant-Gorgia AE, Balant LP. Therapeutic drug monitoring of olanzapine: The combined effect of age, gender, smoking, and comedication. Ther Drug Monit 2003; 25: 46-53

[405] Gex-Fabry M, Balant-Gorgia AE, Balant LP et al. Time course of clinical response to venlafaxine: Relevance of plasma level and chirality. Eur J Clin Pharmacol 2004; 59: 883-891

[406] Gex-Fabry M, Gervasoni N, Eap CB et al. Time course of response to paroxetine: Influence of plasma level. Prog Neuropsychopharmacol Biol Psychiatry 2007; 31: 892-900

[407] Ghosal A, Yuan Y, Tong W et al. Characterization of human liver enzymes involved in the biotransformation of boceprevir, a hepatitis C virus protease inhibitor. Drug Metab Dispos 2011; 39: 510-521

[408] Gidal BE, Majid O, Ferry J et al. The practical impact of altered dosing on perampanel plasma concentrations: Pharmacokinetic modeling from clinical studies. Epilepsy Behav 2014; 35: 6-12

[409] Giessmann T, May K, Modess C et al. Carbamazepine regulates intestinal P-glycoprotein and multidrug resistance protein MRP2 and influences disposition of talinolol in humans. Clin Pharmacol Ther 2004; 76: 192-200

[410] Gilles M, Deuschle M, Kellner S et al. Paroxetine serum concentrations in depressed patients and response to treatment. Pharmacopsychiatry 2005; 38: 118-121

[411] Gillman PK. Advances pertaining to the pharmacology and interactions of irreversible nonselective monoamine oxidase inhibitors. J Clin Psychopharmacol 2011; 31: 66-74

[412] Gillman PK. Tricyclic antidepressant pharmacology and therapeutic drug interactions updated. Br J Pharmacol 2007; 151: 737-748

[413] Gjestad C, Westin AA, Skogvoll E et al. Effect of proton pump inhibitors on the serum concentrations of the selective serotonin reuptake inhibitors citalopram, escitalopram, and sertraline. Ther Drug Monit 2015; 37: 90-97

[414] Glassman AH, Perel JM, Shostak M et al. Clinical implications of imipramine plasma levels for depressive illness. Arch Gen Psychiatry 1977; 34: 197-204

[415] Glauser TA, Pippenger CE. Controversies in blood-level monitoring: Reexamining its role in the treatment of epilepsy. Epilepsia 2000; 41: (Suppl 8): S6-15

[416] Gleason MM, Egger HL, Emslie G] et al. Psychopharmacological treatment for very young children: Contexts and guidelines. J Am Acad Child Adolesc Psychiatry 2007; 46: 1532-1572

[417] Glotzbach RK, Preskorn SH. Brain concentrations of tricyclic antidepressants: Single-dose kinetics and relationship to plasma concentrations in chronically dosed rats. Psychopharmacology (Berl) 1982; 78: 25-27 
[418] Glue P, Cape G, Tunnicliff D et al. Switching opioid-dependent patients from methadone to morphine: Safety, tolerability, and methadone pharmacokinetics. J Clin Pharmacol 2016; 56: 960-965

[419] Gomolin IH, Smith C, Jeitner TM. Once-daily memantine: Pharmacokinetic and clinical considerations. J Am Geriatr Soc 2010; 58: 1812-1813

[420] Gonzalez JP, Brogden RN. Naltrexone. A review of its pharmacodynamic and pharmacokinetic properties and therapeutic efficacy in the management of opioid dependence. Drugs 1988; 35: 192-213

[421] Goodnick PJ, Dominguez RA, DeVane CL et al. Bupropion slowrelease response in depression: Diagnosis and biochemistry. Biol Psychiatry 1998; 44: 629-632

[422] Goole J, Van Gansbeke B, Pilcer G et al. Pharmacoscintigraphic and pharmacokinetic evaluation on healthy human volunteers of sustained-release floating minitablets containing levodopa and carbidopa. Int J Pharm 2008; 364: 54-63

[423] Gram LF, Guentert TW, Grange S et al. Moclobemide, a substrate of CYP2C19 and an inhibitor of CYP2C19, CYP2D6, and CYP1A2: A panel study. Clin Pharmacol Ther 1995; 57: 670-677

[424] Grandjean EM, Aubry JM. Lithium: Updated human knowledge using an evidence-based approach. Part II: Clinical pharmacology and therapeutic monitoring. CNS Drugs 2009; 23: 331-349

[425] Grangeon A, Gravel S, Gaudette F et al. Highly sensitive LC-MS/MS methods for the determination of seven human CYP450 activities using small oral doses of probe-drugs in human. J Chromatogr B Analyt Technol Biomed Life Sci 2017; 1040: 144-158

[426] Granneman GR, Braeckman RA, Locke CS et al. Effect of zileuton on theophylline pharmacokinetics. Clin Pharmacokinet 1995; 29: (Suppl 2): 77-83

[427] Grasela TH, Fiedler-Kelly JB, Salvadori C et al. Predictive performance of population pharmacokinetic parameters of tianeptine as applied to plasma concentrations from a post-marketing study. Eur J Clin Pharmacol 1993; 45: 123-128

[428] Grasmader K, Verwohlt PL, Kuhn KU et al. Relationship between mirtazapine dose, plasma concentration, response, and side effects in clinical practice. Pharmacopsychiatry 2005; 38: 113-117

[429] Greenbaum L, Smith RC, Rigbi A et al. Further evidence for association of the RGS2 gene with antipsychotic-induced parkinsonism: protective role of a functional polymorphism in the 3'-untranslated region. Pharmacogenomics J 2009; 9: 103-110

[430] Greenbaum L, Strous RD, Kanyas K et al. Association of the RGS2 gene with extrapyramidal symptoms induced by treatment with antipsychotic medication. Pharmacogenet Genomics 2007; 17 : 519-528

[431] Greenblatt DJ, Abernethy DR, Divoll M et al. Pharmacokinetic properties of benzodiazepine hypnotics. J Clin Psychopharmacol 1983; 3: 129-132

[432] Greenblatt DJ, Blaskovich PD, Nuwayser ES et al. Clonazepam pharmacokinetics: Comparison of subcutaneous microsphere injection with multiple-dose oral administration. J Clin Pharmacol 2005; 45: 1288-1293

[433] Greenblatt DJ, Divoll M, Harmatz JS et al. Kinetics and clinical effects of flurazepam in young and elderly noninsomniacs. Clin Pharmacol Ther 1981; 30: 475-486

[434] Greenblatt DJ, Divoll M, Harmatz JS et al. Oxazepam kinetics: Effects of age and sex. J Pharmacol Exp Ther 1980; 215: 86-91

[435] Greenblatt DJ, Ehrenberg BL, Gunderman J et al. Pharmacokinetic and electroencephalographic study of intravenous diazepam, midazolam, and placebo. Clin Pharmacol Ther 1989; 45: 356-365

[436] Greenblatt DJ, Gan L, Harmatz JS et al. Pharmocokinetics and pharmacodynamics of single-dose triazolam: Electroencephalography compared with the Digit-Symbol Substitution Test. Br J Clin Pharmacol 2005; 60: 244-248
[437] Greenblatt DJ, Harmatz JS, Friedman H et al. A large-sample study of diazepam pharmacokinetics. Ther Drug Monit 1989; 11: 652-657

[438] Greenblatt DJ, Harmatz JS, von Moltke LL et al. Comparative kinetics and dynamics of zaleplon, zolpidem, and placebo. Clin Pharmacol Ther 1998; 64: 553-561

[439] Greenblatt DJ, Harmatz JS, von Moltke LL et al. Age and gender effects on the pharmacokinetics and pharmacodynamics of triazolam, a cytochrome P450 3A substrate. Clin Pharmacol Ther 2004; 76: 467-479

[440] Greenblatt DJ, Shader RI, Franke K et al. Pharmacokinetics and bioavailability of intravenous, intramuscular, and oral lorazepam in humans. J Pharm Sci 1979; 68: 57-63

[441] Greenblatt DJ, von Moltke LL, Ehrenberg BL et al. Kinetics and dynamics of lorazepam during and after continuous intravenous infusion. Crit Care Med 2000; 28: 2750-2757

[442] Greenblatt DJ, von Moltke LL, Harmatz JS et al. Short-term exposure to low-dose ritonavir impairs clearance and enhances adverse effects of trazodone. J Clin Pharmacol 2003; 43: 414-422

[443] Greig SL.Brexpiprazole First Global Approval. Drugs 2015; 75 : 1687-1697

[444] Greil W, Haberle A, Haueis P et al. Pharmacotherapeutic trends in 2231 psychiatric inpatients with bipolar depression from the International AMSP Project between 1994 and 2009. J Affect Disord 2012; 136: 534-542

[445] Greil W, Haberle A, Schuhmann T et al. Age and adverse drug reactions from psychopharmacological treatment: Data from the AMSP drug surveillance programme in Switzerland. Swiss Med Wkly 2013; 143: w13772

[446] Greiner C, Hiemke C, Bader W et al. Determination of citalopram and escitalopram together with their active main metabolites desmethyl(es-)citalopram in human serum by column-switching high performance liquid chromatography (HPLC) and spectrophotometric detection. J Chromatogr B Analyt Technol Biomed Life Sci 2007; 848: 391-394

[447] Gressier F, Porcelli S, Calati R et al. Pharmacogenetics of clozapine response and induced weight gain: A comprehensive review and meta-analysis. Eur Neuropsychopharmacol 2016; 26: 163-185

[448] Grimaldi R, Perucca E, Ruberto G et al. Pharmacokinetic and pharmacodynamic studies following the intravenous and oral administration of the antiparkinsonian drug biperiden to normal subjects. Eur J Clin Pharmacol 1986; 29: 735-737

[449] Grislain L, Gele P, Bertrand M et al. The metabolic pathways of tianeptine, a new antidepressant, in healthy volunteers. Drug Metab Dispos 1990; 18: 804-808

[450] Grohmann R, Engel RR, Ruther E et al. The AMSP drug safety program: methods and global results. Pharmacopsychiatry 2004; 37 : (Suppl 1): S4-11

[451] Grover CA, Flaherty B, Lung D et al. Significant toxicity in a young female after low-dose tricyclic antidepressant ingestion. Pediatr Emerg Care 2012; 28: 1066-1069

[452] Grözinger M, Härtter S, Hiemke C et al. Oxybutynin enhances the metabolism of clomipramine and dextrorphan possibly by induction of a cytochrome P450 isoenzyme. J Clin Psychopharmacol 1999; 19: 287-289

[453] Gründer G, Baumann P, Conca A et al. Therapeutic drug monitoring in psychiatry. A brief summary of the new consensus paper by the task force on TDM of the AGNP. Nervenarzt 2014; 85: 847-855

[454] Gründer G, Carlsson A, Wong DF. Mechanism of new antipsychotic medications: Occupancy is not just antagonism. Arch Gen Psychiatry 2003; 60: 974-977

[455] Gründer G, Fellows C, Janouschek H et al. Brain and plasma pharmacokinetics of aripiprazole in patients with schizophrenia: An [18F]fallypride PET study. Am J Psychiatry 2008; 165: 988-995 
[456] Gründer G, Hiemke C, Paulzen M et al. Therapeutic plasma concentrations of antidepressants and antipsychotics: Lessons from PET imaging. Pharmacopsychiatry 2011; 44: 236-248

[457] Gründer G, Hippius H, Carlsson A. The 'atypicality' of antipsychotics: A concept re-examined and re-defined. Nat Rev Drug Discov 2009; 8: 197-202

[458] Guay DR. Rasagiline (TVP-1012): a new selective monoamine oxidase inhibitor for Parkinson's disease. Am J Geriatr Pharmacother 2006; 4: 330-346

[459] Guberman A, Couture M, Blaschuk K et al. Add-on trial of clobazam in intractable adult epilepsy with plasma level correlations. Can J Neurol Sci 1990; 17: 311-316

[460] Gunes A, Bilir E, Zengil $\mathrm{H}$ et al. Inhibitory effect of valproic acid on cytochrome $\mathrm{P} 4502 \mathrm{C} 9$ activity in epilepsy patients. Basic Clin Pharmacol Toxicol 2007; 100: 383-386

[461] Gunes A, Spina E, Dahl ML et al. ABCB1 polymorphisms influence steady-state plasma levels of 9-hydroxyrisperidone and risperidone active moiety. Ther Drug Monit 2008; 30: 628-633

[462] Guo W, Guo GX, Sun C et al. Therapeutic drug monitoring of psychotropic drugs in China: a nationwide survey. Ther Drug Monit 2013; 35: 816-822

[463] Gupta N. Guidelines for lithium monitoring: Are they ideal? Acta Psychiatr Scand 2001; 104: 76-77

[464] Gupta RN, Dziurdzy SA. Therapeutic monitoring of sertraline. Clin Chem 1994; 40: 498-499

[465] Gupta SK, Shah JC, Hwang SS. Pharmacokinetic and pharmacodynamic characterization of OROS and immediate-release amitriptyline. $\mathrm{Br}$ J Clin Pharmacol 1999; 48: 71-78

[466] Gutmann H, Poller B, Buter KB et al. Hypericum perforatum: which constituents may induce intestinal MDR1 and CYP3A4 mRNA expression? Planta Med 2006; 72: 685-690

[467] Guy W.editor. ECDEU Assessment Manual for Psychopharmacology. Rockville, MD: U.S. Department of Health, Education, and Welfare; 1976

[468] Haberstroh J, Hampel H, Pantel J. Optimal management of Alzheimer's disease patients: Clinical guidelines and family advice. Neuropsychiatr Dis Treat 2010; 6: 243-253

[469] Haen E. Der TDM-Befund. Psychopharmakotherapie 2012; 19: 129-134

[470] Haen E. Therapeutic drug monitoring in pharmacovigilance and pharmacotherapy safety. Pharmacopsychiatry 2011; 44: 254-258

[471] Haen E, Greiner C, Bader W et al. Expanding therapeutic reference ranges using dose-related reference ranges. Nervenarzt 2008; 79: 558-566

[472] Haen E, Koeber R, Klunemann HH et al. Implementation of a cost-effective HPLC/UV approach for routine medical quantification of memantine in human serum. Ther Drug Monit 2012; 34: 702-712

[473] Haider B, Schmidt R, Schweiger C et al. Medication adherence in patients with dementia: An Austrian cohort study. Alzheimer Dis Assoc Disord 2014; 28: 128-133

[474] Hailey DM, Baird ES. Plasma concentrations of medazepam and its metabolites after oral administration. Br J Anaesth 1979; 51 : 493-496

[475] Hakooz NM. Caffeine metabolic ratios for the in vivo evaluation of CYP1A2, N-acetyltransferase 2, xanthine oxidase and CYP2A6 enzymatic activities. Curr Drug Metab 2009; 10: 329-338

[476] Hallett C, Dean BC. Bromazepam: acute benefit-risk assessment in general practice. Curr Med Res Opin 1984; 8: 683-688

[477] Hallinan R, Crettol S, Agho K et al. Cannabis and benzodiazepines as determinants of methadone trough plasma concentration variability in maintenance treatment: a transnational study. Eur J Clin Pharmacol 2009; 65: 1113-1120
[478] Hamelin BA, Allard S, Laplante L et al. The effect of timing of a standard meal on the pharmacokinetics and pharmacodynamics of the novel atypical antipsychotic agent ziprasidone. Pharmacotherapy 1998; 18: 9-15

[479] Hammarberg A, Beck O, Eksborg S et al. Acamprosate determinations in plasma and cerebrospinal fluid after multiple dosing measured by liquid chromatography-mass spectroscopy: A pharmacokinetic study in healthy volunteers. Ther Drug Monit 2010 32: $489-496$

[480] Hammarberg A, Jayaram-Lindstrom N, Beck O et al. The effects of acamprosate on alcohol-cue reactivity and alcohol priming in dependent patients: A randomized controlled trial. Psychopharmacology (Berl) 2009; 205: 53-62

[481] Hammarlund-Udenaes M. Pharmacokinetic concepts in brain drug delivery. In: Hammarlund-Udenaes M et al. (eds.) Drug Delivery to the Brain, APS Advances in the Pharmaceutical Sciences Series. 10:2014: $127-161$

[482] Handley SA, Bowskill SV, Patel MX et al. Plasma quetiapine in relation to prescribed dose and other factors: Data from a therapeutic drug monitoring service, 2000-2011. Ther Adv Psychopharmacol 2013; 3: 129-137

[483] Hanley JA, McNeil BJ. The meaning and use of the area under a receiver operating characteristic (ROC) curve. Radiology 1982; 143: 29-36

[484] Harden CL, Trifiletti R, Kutt H. Felbamate levels in patients with epilepsy. Epilepsia 1996; 37: 280-283

[485] Härtter S, Dingemanse J, Baier D et al. The role of cytochrome P450 2D6 in the metabolism of moclobemide. Eur Neuropsychopharmacol 1996; 6: 225-230

[486] Härtter S, Hermes B, Szegedi A et al. Automated determination of paroxetine and its main metabolite by column switching and on-line high-performance liquid chromatography. Ther Drug Monit 1994; 16: 400-406

[487] Härtter S, Hiemke C. Column switching and high-performance liquid chromatography in the analysis of amitriptyline, nortriptyline and hydroxylated metabolites in human plasma or serum. J Chromatogr 1992; 578: 273-282

[488] Härtter S, Tybring G, Friedberg T et al. The N-demethylation of the doxepin isomers is mainly catalyzed by the polymorphic CYP2C19. Pharm Res 2002; 19: 1034-1037

[489] Härtter S, Wang X, Weigmann $\mathrm{H}$ et al. Differential effects of fluvoxamine and other antidepressants on the biotransformation of melatonin. J Clin Psychopharmacol 2001; 21: 167-174

[490] Härtter S, Weigmann H, Hiemke C. Automated determination of reboxetine by high-performance liquid chromatography with column-switching and ultraviolet detection. J Chromatogr B Biomed Sci Appl 2000; 740: 135-140

[491] Härtter S, Wetzel H, Hiemke C. Automated determination of fluvoxamine in plasma by column-switching high-performance liquid chromatography. Clin Chem 1992; 38: 2082-2086

[492] Hasselstrom J, Linnet K. Quetiapine serum concentrations in psychiatric patients: The influence of comedication. Ther Drug Monit 2004; 26: 486-491

[493] Hassler F, Glaser T, Pap AF et al. A double-blind placebo-controlled discontinuation study of zuclopenthixol for the treatment of aggressive disruptive behaviours in adults with mental retardation - secondary parameter analyses. Pharmacopsychiatry 2008; 41: 232-239

[494] Hassler F, Reis O. Pharmacotherapy of disruptive behavior in mentally retarded subjects: A review of the current literature. Dev Disabil Res Rev 2010; 16: 265-272

[495] Hattori N, Hasegawa K, Sakamoto T. Pharmacokinetics and effect of food after oral administration of prolonged-release tablets of ropinirole hydrochloride in Japanese patients with Parkinson's disease. J Clin Pharm Ther 2012; 37: 571-577 
[496] Havemann-Reinecke U, Küfner $\mathrm{H}$, Schneider $\mathrm{U}$ et al. Postakutbehandlung bei Störungen durch Opioide. In: Schmidt LG, Gastpar M, Falkai P et al.(Eds.) Evidenzbasierte Suchtmedizin. AWMF S2 Behandungsleitlinie Suchtbezogene Störungen. Köln: Dt. Ärzte-Verlag; 2006: 193-239

[497] Haymond ], Ensom MH. Does valproic acid warrant therapeutic drug monitoring in bipolar affective disorder? Ther Drug Monit 2010; 32: $19-29$

[498] Hazell P, Becker K, Nikkanen EA et al. Relationship between atomoxetine plasma concentration, treatment response and tolerability in attention-deficit/hyperactivity disorder and comorbid oppositional defiant disorder. Atten Defic Hyperact Disord 2009; 1: 201-210

[499] Hefner G, Brueckner A, Hiemke C et al. Therapeutic drug monitoring for patients with Alzheimer dementia to improve treatment with donepezil. Ther Drug Monit 2015; 37: 353-361

[500] Hefner G, Laib AK, Sigurdsson $\mathrm{H}$ et al. The value of drug and metabolite concentration in blood as a biomarker of psychopharmacological therapy. Int Rev Psychiatry 2013; 25: 494-508

[501] Hefner G, Shams ME, Unterecker S et al. Inflammation and psychotropic drugs: The relationship between C-reactive protein and antipsychotic drug levels. Psychopharmacology (Berl) 2016; 233: 1695-1705

[502] Hefner G, Unterecker S, Shams ME et al. Melperone but not bisoprolol or metoprolol is a clinically relevant inhibitor of CYP2D6: evidence from a therapeutic drug monitoring survey. J Neural Transm (Vienna) 2015; 122: 1609-1617

[503] Hegerl U, Bottlender R, Gallinat J et al. The serotonin syndrome scale: first results on validity. Eur Arch Psychiatry Clin Neurosci 1998; 248: 96-103

[504] Heikkinen H, Saraheimo M, Antila S et al. Pharmacokinetics of entacapone, a peripherally acting catechol-O-methyltransferase inhibitor, in man. A study using a stable isotope techique. Eur J Clin Pharmacol 2001; 56: 821-826

[505] Hejna P, Ublova M, Vorisek V. Black esophagus: acute esophageal necrosis in fatal haloperidol intoxication. J Forensic Sci 2013; 58: 1367-1369

[506] Heller S, Hiemke C, Stroba G et al. Assessment of storage and transport stability of new antidepressant and antipsychotic drugs for a nationwide TDM service. Ther Drug Monit 2004; 26: 459-461

[507] Hellriegel ET, Arora S, Nelson M et al. Steady-state pharmacokinetics and tolerability of modafinil administered alone or in combination with dextroamphetamine in healthy volunteers. J Clin Pharmacol 2002; 42: 450-460

[508] Hendershot CS. Pharmacogenetic approaches in the treatment of alcohol use disorders: Addressing clinical utility and implementation thresholds. Addict Sci Clin Pract 2014; 9: 20

[509] Hendset M, Hermann M, Lunde $H$ et al. Impact of the CYP2D6 genotype on steady-state serum concentrations of aripiprazole and dehydroaripiprazole. Eur J Clin Pharmacol 2007; 63: 1147-1151

[510] Herman BD, Fleishaker JC, Brown MT. Ketoconazole inhibits the clearance of the enantiomers of the antidepressant reboxetine in humans. Clin Pharmacol Ther 1999; 66: 374-379

[511] Herman RJ, Wilkinson GR. Disposition of diazepam in young and elderly subjects after acute and chronic dosing. Br J Clin Pharmacol 1996; 42: 147-155

[512] Hermann M, Hendset M, Fosaas K et al. Serum concentrations of venlafaxine and its metabolites $\mathrm{O}$-desmethylvenlafaxine and $\mathrm{N}$-desmethylvenlafaxine in heterozygous carriers of the $\mathrm{CY}$ P2D6 ${ }^{*} 3,{ }^{*} 4$ or ${ }^{*} 5$ allele. Eur J Clin Pharmacol 2008; 64: 483-487

[513] Hermida J, Paz E, Tutor JC. Clozapine and norclozapine concentrations in serum and plasma samples from schizophrenic patients. Ther Drug Monit 2008; 30: 41-45
[514] Hesse LM, He P, Krishnaswamy S et al. Pharmacogenetic determinants of interindividual variability in bupropion hydroxylation by cytochrome P450 2B6 in human liver microsomes. Pharmacogenetics 2004; 14: 225-238

[515] Hicks JK, Bishop JR, Sangkuhl K et al. Clinical Pharmacogenetics Implementation Consortium (CPIC) guideline for CYP2D6 and CYP2C19 genotypes and dosing of selective serotonin reuptake inhibitors. Clin Pharmacol Ther 2015; 98: 127-134

[516] Hicks JK, Sangkuhl K, Swen J] et al. Clinical pharmacogenetics implementation consortium guideline (CPIC) for CYP2D6 and CYP2C19 genotypes and dosing of tricyclic antidepressants: 2016 update. Clin Pharmacol Ther 2017; 102: 37-44

[517] Hicks JK, Swen J], Thorn CF et al. Clinical Pharmacogenetics Implementation Consortium guideline for CYP2D6 and CYP2C19 genotypes and dosing of tricyclic antidepressants. Clin Pharmacol Ther 2013; 93: 402-408

[518] Hiemke C. Clinical utility of drug measurement and pharmacokinetics: therapeutic drug monitoring in psychiatry. Eur J Clin Pharmacol 2008; 64: 159-166

[519] Hiemke C. Consensus Guideline Based Therapeutic Drug Monitoring (TDM) in Psychiatry and Neurology. Curr Drug Deliv 2016; 13: 353-361

[520] Hiemke C. Therapeutic drug monitoring in neuropsychopharmacology: does it hold its promises? Eur Arch Psychiatry Clin Neurosci 2008; 258: (Suppl 1): 21-27

[521] Hiemke C, Baumann P, Bergemann N et al. AGNP-Konsensus-Leitlinie für therapeutisches Drug-Monitoring in der Psychiatrie. Update 2011. Psychopharmakotherapie 2012; 19: 91-122

[522] Hiemke C, Baumann P, Bergemann N et al. Linee guida della consensus conference dell'ANGP per il monitoraggio plasmatico dei farmaci (TDM) in psichiatria. Aggiornamento 2011. Journal of Psychopathology 2014; 20: 286-321

[523] Hiemke C, Baumann P, Bergemann N et al. The TDM group of the AGNP Az AGNP konszenzuson alapuló irányelvei a terápiás gyógyszerszint montorozásához a pszichiátriában. Psychiatria Hungarica 2012; 27: (Suppl 1): 7-64

[524] Hiemke C, Baumann P, Bergemann N et al. AGNP consensus guidelines for therapeutic drug monitoring in psychiatry: Update 2011. Pharmacopsychiatry 2011; 44: 195-235

[525] Hiemke C, Dragicevic A, Gründer G et al. Therapeutic monitoring of new antipsychotic drugs. Ther Drug Monit 2004; 26: 156-160

[526] Hiemke C, Härtter S. Pharmacokinetics of selective serotonin reuptake inhibitors. Pharmacol Ther 2000; 85: 11-28

[527] Hiemke C, Shams M. Phenotyping and genotyping of drug metabolism to guide pharmacotherapy in psychiatry. Curr Drug Deliv 2013; 10: 46-53

[528] Higuchi H, Yoshida K, Takahashi H et al. Milnacipran plasma levels and antidepressant response in Japanese major depressive patients. Hum Psychopharmacol 2003; 18: 255-259

[529] Hildebrand M, Hellstern A, Humpel M et al. Plasma levels and urinary excretion of lormetazepam in patients with liver cirrhosis and in healthy volunteers. Eur J Drug Metab Pharmacokinet 1990; 15: 19-26

[530] Hirsch L], Arif H, Buchsbaum R et al. Effect of age and comedication on levetiracetam pharmacokinetics and tolerability. Epilepsia 2007; 48: 1351-1359

[531] Hirsch LJ, Weintraub D, Du Y et al. Correlating lamotrigine serum concentrations with tolerability in patients with epilepsy. Neurology 2004; 63: 1022-1026

[532] Hodge LS, Tracy TS. Alterations in drug disposition during pregnancy: implications for drug therapy. Expert Opin Drug Metab Toxicol 2007; 3: 557-571 
[533] Hohmann N, Haefeli WE, Mikus G. Use of microdose phenotyping to individualise dosing of patients. Clin Pharmacokinet 2015; 54: 893-900

[534] Hoiseth G, Haslemo T, Uthus LH et al. Effect of CYP2B6 * 6 on Steady-state serum concentrations of bupropion and hydroxybupropion in psychiatric patients: A study based on therapeutic drug monitoring data. Ther Drug Monit 2015; 37: 589-593

[535] Hojer J, Hulting J, Salmonson H. Fatal cardiotoxicity induced by venlafaxine overdosage. Clin Toxicol (Phila) 2008; 46: 336-337

[536] Holbrook JM, Parks-Veal P, Mimbs ]. Clinical monitoring guidelines for neuroleptic and antidepressant drugs. Central State Hospital, Milledgeville, Georgia. Hosp Pharm 1991; 26 (783/784): 787-793

[537] Holt S, Schmiedl S, Thurmann PA. Potentially inappropriate medications in the elderly: The PRISCUS list. Dtsch Arztebl Int 2010; 107: $543-551$

[538] Holzer L, Preuss U, Baumgartner L et al. Quetiapine in adolescents with non-affective psychotic disorders: An open-label trial. Pharmacopsychiatry 2011; 44: 87-95

[539] Hooper WD, Dickinson RG, Dunstan PR et al. Oxcarbazepine: preliminary clinical and pharmacokinetic studies on a new anticonvulsant. Clin Exp Neurol 1987; 24: 105-112

[540] Horadam VW, Sharp JG, Smilack JD et al. Pharmacokinetics of amantadine hydrochloride in subjects with normal and impaired renal function. Ann Intern Med 1981; 94: 454-458

[541] Hou L, Heilbronner U, Degenhardt F et al. Genetic variants associated with response to lithium treatment in bipolar disorder: A genomewide association study. Lancet 2016; 387: 1085-1093

[542] Hrdina PD, Lapierre YD. Plasma levels of maprotiline and zimelidine and their relationship to clinical response in depressed patients. Ther Drug Monit 1986; 8: 400-406

[543] Hsieh YH, Yang YH, Yeh HH et al. Simultaneous determination of galantamine, rivastigmine and NAP 226-90 in plasma by MEKC and its application in Alzheimer's disease. Electrophoresis 2009; 30: 644-653

[544] Huezo-Diaz P, Perroud N, Spencer EP et al. CYP2C19 genotype predicts steady state escitalopram concentration in GENDEP. J Psychopharmacol 2012; 26: 398-407

[545] Hughes J, Gill AM, Mulhearn $\mathrm{H}$ et al. Steady-state plasma concentrations of midazolam in critically ill infants and children. Ann Pharmacother 1996; 30: 27-30

[546] Hui WK, Mitchell LB, Kavanagh KM et al. Melperone: Electrophysiologic and antiarrhythmic activity in humans. J Cardiovasc Pharmacol 1990; 15: 144-149

[547] Huttunen KM, Raunio H, Rautio J. Prodrugs-from serendipity to rational design. Pharmacol Rev 2011; 63: 750-771

[548] Hvenegaard MG, Bang-Andersen B, Pedersen $\mathrm{H}$ et al. Identification of the cytochrome P450 and other enzymes involved in the in vitro oxidative metabolism of a novel antidepressant, Lu AA21004. Drug Metab Dispos 2012; 40: 1357-1365

[549] Ignjatovic AR, Miljkovic B, Todorovic D et al. Evaluation of singlepoint sampling strategies for the estimation of moclobemide exposure in depressive patients. J Clin Pharmacol 2011; 51: 661-671

[550] Ilett KF, Blythe TH, Hackett LP et al. Plasma concentrations of dothiepin and its metabolites are not correlated with clinical efficacy in major depressive illness. Ther Drug Monit 1993; 15: 351-357

[551] Ingman K, Hagelberg N, Aalto S et al. Prolonged central mu-opioid receptor occupancy after single and repeated nalmefene dosing. Neuropsychopharmacology 2005; 30: 2245-2253

[552] Inui N, Akamatsu T, Uchida S et al. Chronological effects of rifampicin discontinuation on cytochrome P450 activity in healthy Japanese volunteers, using the cocktail method. Clin Pharmacol Ther 2013; 94: $702-708$

[553] Investigators G, Investigators M, Investigators SD. Common genetic variation and antidepressant efficacy in major depressive disorder: A meta-analysis of three genome-wide pharmacogenetic studies. Am J Psychiatry 2013; 170: 207-217

[554] Isacsson G, Holmgren P, Druid $\mathrm{H}$ et al. The utilization of antidepressants--a key issue in the prevention of suicide: An analysis of 5281 suicides in Sweden during the period 1992-1994. Acta Psychiatr Scand 1997; 96: 94-100

[555] Isbister GK, Hackett LP, Dawson AH et al. Moclobemide poisoning: toxicokinetics and occurrence of serotonin toxicity. $\mathrm{Br}$ ] Clin Pharmacol 2003; 56: 441-450

[556] Ishida M, Otani K, Kaneko S et al. Effects of various factors on steady state plasma concentrations of trazodone and its active metabolite m-chlorophenylpiperazine. Int Clin Psychopharmacol 1995; 10: 143-146

[557] Iwamoto K, Kawamura Y, Takahashi M et al. Plasma amitriptyline level after acute administration, and driving performance in healthy volunteers. Psychiatry Clin Neurosci 2008; 62: 610-616

[558] Iwersen S, Schmoldt A. One fatal and one nonfatal intoxication with tranylcypromine. Absence of amphetamines as metabolites. J Anal Toxicol 1996; 20: 301-304

[559] Jaanson P, Marandi T, Kiivet RA et al. Maintenance therapy with zuclopenthixol decanoate: associations between plasma concentrations, neurological side effects and CYP2D6 genotype. Psychopharmacology (Berl) 2002; 162: 67-73

[560] Jackson A. Impact of release mechanism on the pharmacokinetic performance of PAUC metrics for three methylphenidate products with complex absorption. Pharm Res 2014; 31: 173-181

[561] Jackson SH, Jansen PA, Mangoni AA. Off-label prescribing in older patients. Drugs Aging 2012; 29: 427-434

[562] Jacob S, Nair AB. An Updated Overview on Therapeutic Drug Monitoring of Recent Antiepileptic Drugs. Drugs R D 2016; 16: 303-316

[563] Jacqmin P, Ansseau M. Comparison of sublingual and oral prazepam in normal subjects. II. Pharmacokinetic and pharmacodynamic data. Neuropsychobiology 1988; 19: 186-191

[564] Jakubovski E, Varigonda AL, Freemantle $N$ et al. Systematic review and meta-analysis: dose-response relationship of selective serotonin reuptake inhibitors in major depressive disorder. Am J Psychiatry 2016; 173: 174-183

[565] Jang SH, Yan Z, Lazor JA. Therapeutic drug monitoring: A patient management tool for precision medicine. Clin Pharmacol Ther 2016; 99: $148-150$

[566] Jann MW, Shirley KL, Small GW. Clinical pharmacokinetics and pharmacodynamics of cholinesterase inhibitors. Clin Pharmacokinet 2002; 41: 719-739

[567] Jaquenoud Sirot E, Harenberg S, Vandel P et al. Multicenter study on the clinical effectiveness, pharmacokinetics, and pharmacogenetics of mirtazapine in depression. J Clin Psychopharmacol 2012; 32: 622-629

[568] Jaquenoud Sirot E, Knezevic B, Morena GP et al. ABCB1 and cytochrome P450 polymorphisms: Clinical pharmacogenetics of clozapine. J Clin Psychopharmacol 2009; 29: 319-326

[569] Jaquenoud Sirot E, van der Velden JW, Rentsch K et al. Therapeutic drug monitoring and pharmacogenetic tests as tools in pharmacovigilance. Drug Saf 2006; 29: 735-768

[570] Jefferson JW, Pradko JF, Muir KT. Bupropion for major depressive disorder: Pharmacokinetic and formulation considerations. Clin Ther 2005; 27: 1685-1695 
[571] Jenner P, Konen-Bergmann M, Schepers C et al. Pharmacokinetics of a once-daily extended-release formulation of pramipexole in healthy male volunteers: three studies. Clin Ther 2009; 31: 2698-2711

[572] Jeppesen U, Gram LF, Vistisen K et al. Dose-dependent inhibition of CYP1A2, CYP2C19 and CYP2D6 by citalopram, fluoxetine, fluvoxamine and paroxetine. Eur J Clin Pharmacol 1996; 51: 73-78

[573] Jerling M, Bertilsson L, Sjoqvist F. The use of therapeutic drug monitoring data to document kinetic drug interactions: An example with amitriptyline and nortriptyline. Ther Drug Monit 1994; 16: 1-12

[574] Jerling M, Dahl ML, Aberg-Wistedt A et al. The CYP2D6 genotype predicts the oral clearance of the neuroleptic agents perphenazine and zuclopenthixol. Clin Pharmacol Ther 1996; 59: 423-428

[575] Jerling M, Merle Y, Mentre F et al. Population pharmacokinetics of clozapine evaluated with the nonparametric maximum likelihood method. Br J Clin Pharmacol 1997; 44: 447-453

[576] Jerling M, Merle Y, Mentre F et al. Population pharmacokinetics of nortriptyline during monotherapy and during concomitant treatment with drugs that inhibit CYP2D6--an evaluation with the nonparametric maximum likelihood method. Br J Clin Pharmacol 1994; 38 : 453-462

[577] Ji P, Damle B, Xie J et al. Pharmacokinetic interaction between efavirenz and carbamazepine after multiple-dose administration in healthy subjects. J Clin Pharmacol 2008; 48: 948-956

[578] Ji Y, Schaid DJ, Desta Z et al. Citalopram and escitalopram plasma drug and metabolite concentrations: genome-wide associations. $\mathrm{Br}$ J Clin Pharmacol 2014; 78: 373-383

[579] Jia C, Luo L, Kurogi K et al. Identification of the Human SULT enzymes involved in the metabolism of rotigotine. J Clin Pharmacol 2016; 56 : 754-760

[580] Jiang F, Kim HD, Na HS et al. The influences of CYP2D6 genotypes and drug interactions on the pharmacokinetics of venlafaxine: Exploring predictive biomarkers for treatment outcomes. Psychopharmacology (Berl) 2015; 232: 1899-1909

[581] Jimmink A, Caminada K, Hunfeld NG et al. Clinical toxicology of citalopram after acute intoxication with the sole drug or in combination with other drugs: Overview of 26 cases. Ther Drug Monit 2008; 30: 365-371

[582] Jin Y, Pollock BG, Coley K et al. Population pharmacokinetics of perphenazine in schizophrenia patients from CATIE: Impact of race and smoking. J Clin Pharmacol 2010; 50: 73-80

[583] Jin Y, Pollock BG, Frank E et al. Effect of age, weight, and CYP2C19 genotype on escitalopram exposure. J Clin Pharmacol 2010; 50: $62-72$

[584] Jin Y, Pollock BG, Frank E et al. The effect of reporting methods for dosing times on the estimation of pharmacokinetic parameters of escitalopram. J Clin Pharmacol 2009; 49: 176-184

[585] Jochemsen R, Wesselman JG, Hermans J et al. Pharmacokinetics of brotizolam in healthy subjects following intravenous and oral administration. Br J Clin Pharmacol 1983; 16 (Suppl 2): 285S-290S

[586] Johannessen Landmark C, Svendsen T, Dinarevic J et al. The impact of pharmacokinetic interactions with eslicarbazepine acetate versus oxcarbazepine and carbamazepine in clinical practice. Ther Drug Monit 2016; 38: 499-505

[587] Johannessen SI, Tomson T. Pharmacokinetic variability of newer antiepileptic drugs: When is monitoring needed? Clin Pharmacokinet 2006; 45: 1061-1075

[588] Johansson B. A review of the pharmacokinetics and pharmacodynamics of disulfiram and its metabolites. Acta Psychiatr Scand Suppl 1992; 369: 15-26

[589] Johnston JA, Fiedler-Kelly J, Glover ED et al. Relationship between drug exposure and the efficacy and safety of bupropion sustained release for smoking cessation. Nicotine Tob Res 2001; 3: 131-140
[590] Jones AW. Ultra-rapid rate of ethanol elimination from blood in drunken drivers with extremely high blood-alcohol concentrations. Int J Legal Med 2008; 122: 129-134

[591] Jones AW, Holmgren A. Concentrations of diazepam and nordiazepam in 1,000 blood samples from apprehended drivers--therapeutic use or abuse of anxiolytics? J Pharm Pract 2013; 26: 198-203

[592] Jörg ], Pröfrock A. Wirksamkeit und Verträglichkeit der ParkinsonBehandlung mit Amantadinsulfat. Nervenheilkunde 1995; 14: 76-82

[593] Jorga KM, Fotteler B, Heizmann P et al. Pharmacokinetics and pharmacodynamics after oral and intravenous administration of tolcapone, a novel adjunct to Parkinson's disease therapy. Eur J Clin Pharmacol 1998; 54: 443-447

[594] Jorga KM, Larsen JP, Beiske A et al. The effect of tolcapone on the pharmacokinetics of benserazide. Eur J Neurol 1999; 6: 211-219

[595] Jorgensen A. Pharmacokinetic studies in volunteers of intravenous and oral cis (Z)-flupentixol and intramuscular cis (Z)-flupentixol decanoate in Viscoleo. Eur J Clin Pharmacol 1980; 18: 355-360

[596] Jornil J, Jensen KG, Larsen F et al. Identification of cytochrome P450 isoforms involved in the metabolism of paroxetine and estimation of their importance for human paroxetine metabolism using a population-based simulator. Drug Metab Dispos 2010; 38: 376-385

[597] Jornil J, Jensen KG, Larsen F et al. Risk assessment of accidental nortriptyline poisoning: The importance of cytochrome P450 for nortriptyline elimination investigated using a population-based pharmacokinetic simulator. Eur J Pharm Sci 2011; 44: 265-272

[598] Jornil J, Nielsen TS, Rosendal I et al. A poor metabolizer of both CYP2C19 and CYP2D6 identified by mechanistic pharmacokinetic simulation in a fatal drug poisoning case involving venlafaxine. Forensic Sci Int 2013; 226: e26-e31

[599] Jovanovic M, Sokic D, Grabnar I et al. Population pharmacokinetics of topiramate in adult patients with epilepsy using nonlinear mixed effects modelling. Eur ] Pharm Sci 2013; 50: 282-289

[600] Kagawa S, Mihara K, Nakamura A et al. Relationship between plasma concentrations of lamotrigine and its early therapeutic effect of lamotrigine augmentation therapy in treatment-resistant depressive disorder. Ther Drug Monit 2014; 36: 730-733

[601] Kajosaari LI, Niemi M, Backman JT et al. Telithromycin, but not montelukast, increases the plasma concentrations and effects of the cytochrome P450 3A4 and 2C8 substrate repaglinide. Clin Pharmacol Ther 2006; 79: 231-242

[602] Kandasamy M, Srinivas P, Subramaniam K et al. Differential outcomes from metabolic ratios in the identification of CYP2D6 phenotypes-focus on venlafaxine and O-desmethylvenlafaxine. Eur J Clin Pharmacol 2010; 66: 879-887

[603] Kane JM, Leucht S, Carpenter D et al. The expert consensus guideline series. Optimizing pharmacologic treatment of psychotic disorders. Introduction: Methods, commentary, and summary. J Clin Psychiatry 2003; 64 (Suppl 12): 5-19

[604] Kaplan SA, de Silva JA, Jack ML et al. Blood level profile in man following chronic oral administration of flurazepam hydrochloride. J Pharm Sci 1973; 62: 1932-1935

[605] Kaplan SA, Jack ML, Weinfeld RE et al. Biopharmaceutical and clinical pharmacokinetic profile of bromazepam. J Pharmacokinet Biopharm 1976; 4: 1-16

[606] Karlsson L, Zackrisson AL, Josefsson M et al. Influence of CYP2D6 and CYP2C19 genotypes on venlafaxine metabolic ratios and stereoselective metabolism in forensic autopsy cases. Pharmacogenomics J 2015; 15: 165-171

[607] Karson CN, Newton JE, Livingston R et al. Human brain fluoxetine concentrations. J Neuropsychiatry Clin Neurosci 1993; 5: 322-329

[608] Kasper S, Dotsch M, Kick H et al. Plasma concentrations of fluvoxamine and maprotiline in major depression: Implications on therapeutic efficacy and side effects. Eur Neuropsychopharmacol 1993; 3: 13-21 
[609] Katayama Y, Terao T, Kamei K et al. Therapeutic window of lamotrigine for mood disorders: A naturalistic retrospective study. Pharmacopsychiatry 2014; 47: 111-114

[610] Kato M, Serretti A, Nonen S et al. Genetic variants in combination with early partial improvement as a clinical utility predictor of treatment outcome in major depressive disorder: The result of two pooled RCTs. Transl Psychiatry 2015; 5: e513

[611] Katoh Y, Uchida S, Kawai M et al. Effects of cigarette smoking and cytochrome P450 2D6 genotype on fluvoxamine concentration in plasma of Japanese patients. Biol Pharm Bull 2010; 33: 285-288

[612] Katon W, Cantrell CR, Sokol MC et al. Impact of antidepressant drug adherence on comorbid medication use and resource utilization. Arch Intern Med 2005; 165: 2497-2503

[613] Kaufman E, Lamster IB. The diagnostic applications of saliva-a review. Crit Rev Oral Biol Med 2002; 13: 197-212

[614] Kaye CM, Nicholls B. Clinical pharmacokinetics of ropinirole. Clin Pharmacokinet 2000; 39: 243-254

[615] Kemp DE, Ganocy SJ, Brecher M et al. Clinical value of early partial symptomatic improvement in the prediction of response and remission during short-term treatment trials in 3369 subjects with bipolar I or II depression. J Affect Disord 2011; 130: 171-179

[616] Kennedy MC. Post-mortem drug concentrations. Intern Med J 2010; 40: $183-187$

[617] Kerbusch-Herben V, Cleton A, Berwaerts J et al. Effect of carbamazepine on the pharmacokinetics of paliperidone extended-release tablets at steady-state. Clin Pharmacol Drug Dev 2014; 3: 371-377

[618] Kerr BM, Thummel KE, Wurden C] et al. Human liver carbamazepine metabolism. Role of CYP3A4 and CYP2C8 in 10,11-epoxide formation. Biochem Pharmacol 1994; 47: 1969-1979

[619] Kharasch ED, Hankins DC, Jubert C et al. Lack of single-dose disulfiram effects on cytochrome P-450 2C9, 2C19, 2D6, and 3A4 activities: evidence for specificity toward P-450 2E1. Drug Metab Dispos 1999; 27: 717-723

[620] Kharasch ED, Hoffer C, Whittington D et al. Role of P-glycoprotein in the intestinal absorption and clinical effects of morphine. Clin Pharmacol Ther 2003; 74: 543-554

[621] Kharasch ED, Mitchell D, Coles R. Stereoselective bupropion hydroxylation as an in vivo phenotypic probe for cytochrome P4502B6 (CYP2B6) activity. J Clin Pharmacol 2008; 48: 464-474

[622] Kiang TK, Ensom MH, Chang TK. UDP-glucuronosyltransferases and clinical drug-drug interactions. Pharmacol Ther 2005; 106: 97-132

[623] Kiechel JR. Pharmacokinetics and metabolism of guanfacine in man: a review. Br J Clin Pharmacol 1980; 10: (Suppl 1): 25S-32S

[624] Kikkawa H, Maruyama N, Fujimoto $Y$ et al. Single- and multiple-dose pharmacokinetics of the selective nicotinic receptor partial agonist, varenicline, in healthy Japanese adult smokers. J Clin Pharmacol 2011; 51: 527-537

[625] Kim E, Howes OD, Kim BH et al. Predicting brain occupancy from plasma levels using PET: Superiority of combining pharmacokinetics with pharmacodynamics while modeling the relationship. J Cereb Blood Flow Metab 2012; 32: 759-768

[626] Kim JR, Seo HB, Cho JY et al. Population pharmacokinetic modelling of aripiprazole and its active metabolite, dehydroaripiprazole, in psychiatric patients. Br J Clin Pharmacol 2008; 66: 802-810

[627] Kim KA, Lim JL, Kim C et al. Pharmacokinetic comparison of orally disintegrating and conventional donepezil formulations in healthy Korean male subjects: A single-dose, randomized, open-label, 2-sequence, 2-period crossover study. Clin Ther 2011; 33: 965-972

[628] Kinirons MT, O'Mahony MS. Drug metabolism and ageing. $\mathrm{Br}$ ] Clin Pharmacol 2004; 57: 540-544

[629] Kirby B], Collier AC, Kharasch ED et al. Complex drug interactions of HIV protease inhibitors 1: Inactivation, induction, and inhibition of cytochrome P450 3A by ritonavir or nelfinavir. Drug Metab Dispos 2011; 39: 1070-1078

[630] Kirchheiner J. CYP2D6 phenotype prediction from genotype: Which system is the best? Clin Pharmacol Ther 2008; 83: 225-227

[631] Kirchheiner J, Meineke I, Müller $G$ et al. Contributions of CYP2D6, CYP2C9 and CYP2C19 to the biotransformation of E- and Z-doxepin in healthy volunteers. Pharmacogenetics 2002; 12: 571-580

[632] Kirchheiner ], Müller G, Meineke I et al. Effects of polymorphisms in CYP2D6, CYP2C9, and CYP2C19 on trimipramine pharmacokinetics. J Clin Psychopharmacol 2003; 23: 459-466

[633] Kirchheiner J, Nickchen K, Bauer M et al. Pharmacogenetics of antidepressants and antipsychotics: The contribution of allelic variations to the phenotype of drug response. Mol Psychiatry 2004; 9: 442-473

[634] Kirchheiner J, Seeringer A. Clinical implications of pharmacogenetics of cytochrome P450 drug metabolizing enzymes. Biochim Biophys Acta 2007; 1770: 489-494

[635] Kirchherr H, Kuhn-Velten WN. Quantitative determination of forty-eight antidepressants and antipsychotics in human serum by HPLC tandem mass spectrometry: A multi-level, single-sample approach. J Chromatogr B Analyt Technol Biomed Life Sci 2006; 843: $100-113$

[636] Kirkton C, McIntyre IM. Therapeutic and toxic concentrations of mirtazapine. J Anal Toxicol 2006; 30: 687-691

[637] Kirschbaum KM, Müller M], Malevani J et al. Serum levels of aripiprazole and dehydroaripiprazole, clinical response and side effects. World J Biol Psychiatry 2008; 9: 212-218

[638] Kirschbaum KM, Müller M], Zernig G et al. Therapeutic monitoring of aripiprazole by HPLC with column-switching and spectrophotometric detection. Clin Chem 2005; 51: 1718-1721

[639] Kirschbaum KM, Uhr M, Holthoewer D et al. Pharmacokinetics of acute and sub-chronic aripiprazole in P-glycoprotein deficient mice. Neuropharmacology 2010; 59: 474-479

[640] Kirson NY, Weiden P], Yermakov $S$ et al. Efficacy and effectiveness of depot versus oral antipsychotics in schizophrenia: Synthesizing results across different research designs. J Clin Psychiatry 2013; 74: 568-575

[641] Kishimoto T, Robenzadeh A, Leucht C et al. Long-acting injectable vs oral antipsychotics for relapse prevention in schizophrenia: $\mathrm{A}$ meta-analysis of randomized trials. Schizophr Bull 2014; 40: 192-213

[642] Kistrup K, Gerlach J, Aaes-Jorgensen T et al. Perphenazine decanoate and cis(z)-flupentixol decanoate in maintenance treatment of schizophrenic outpatients. Serum levels at the minimum effective dose. Psychopharmacology (Berl) 1991; 105: 42-48

[643] Kitzlerova E, Slavicek J, Pisvejcova K et al. Plasma levels of dosulepine and heart electric field. Physiol Res 2003; 52: 319-325

[644] Kivisto KT, Kroemer HK. Use of probe drugs as predictors of drug metabolism in humans. J Clin Pharmacol 1997; 37: 40S-48S

[645] Kjolbye M, Thomsen K, Rogne T et al. Search for a therapeutic range for serum zuclopenthixol concentrations in schizophrenic patients. Ther Drug Monit 1994; 16: 541-547

[646] Klamerus KJ, Maloney K, Rudolph RL et al. Introduction of a composite parameter to the pharmacokinetics of venlafaxine and its active O-desmethyl metabolite. J Clin Pharmacol 1992; 32: 716-724

[647] Klampfl K, Taurines R, Preuss A et al. Serum concentrations, therapeutic response and side effects in children and adolescents with impulsive-aggressive symptoms during risperidone therapy. Pharmacopsychiatry 2010; 43: 58-65

[648] Kleimann A, Schrader V, Stubner S et al. Psychopharmacological treatment of 1650 in-patients with acute mania-data from the AMSP study. J Affect Disord 2016; 191: 164-171 
[649] Klengel T, Binder EB. Gene x environment interactions in the prediction of response to antidepressant treatment. Int J Neuropsychopharmacol 2013; 16: 701-711

[650] Klinger G, Stahl B, Fusar-Poli P et al. Antipsychotic drugs and breastfeeding. Pediatr Endocrinol Rev 2013; 10: 308-317

[651] Klotz U. Pharmacokinetics and drug metabolism in the elderly. Drug Metab Rev 2009; 41: 67-76

[652] Kodaira H, Kusuhara H, Fujita T et al. Quantitative evaluation of the impact of active efflux by $\mathrm{p}$-glycoprotein and breast cancer resistance protein at the blood-brain barrier on the predictability of the unbound concentrations of drugs in the brain using cerebrospinal fluid concentration as a surrogate. J Pharmacol Exp Ther 2011; 339 : 935-944

[653] Koeber R, Kluenemann HH, Waimer R et al. Implementation of a cost-effective HPLC/UV-approach for medical routine quantification of donepezil in human serum. J Chromatogr B Analyt Technol Biomed Life Sci 2012; 881-882: 1-11

[654] Koelch M, Pfalzer AK, Kliegl K et al. Therapeutic drug monitoring of children and adolescents treated with fluoxetine. Pharmacopsychiatry 2012; 45: 72-76

[655] Koepp M], Patsalos PN, Sander JW. Sulthiame in adults with refractory epilepsy and learning disability: An open trial. Epilepsy Res 2002; 50: 277-282

[656] Kokras N, Dalla C, Papadopoulou-Daifoti Z. Sex differences in pharmacokinetics of antidepressants. Expert Opin Drug Metab Toxicol 2011; 7: 213-226

[657] Kondo T, Otani K, Ishida M et al. Adverse effects of zotepine and their relationship to serum concentrations of the drug and prolactin. Ther Drug Monit 1994; 16: 120-124

[658] Kootstra-Ros JE, Van Weelden M], Hinrichs JW et al. Therapeutic drug monitoring of antidepressants and cytochrome p450 genotyping in general practice. J Clin Pharmacol 2006; 46: 1320-1327

[659] Kornhuber J, Kennepohl EM, Bleich S et al. Memantine pharmacotherapy: A naturalistic study using a population pharmacokinetic approach. Clin Pharmacokinet 2007; 46: 599-612

[660] Kornhuber ], Quack G, Danysz W et al. Therapeutic brain concentration of the NMDA receptor antagonist amantadine. Neuropharmacology 1995; 34: 713-721

[661] Kosaki K, Tamura K, Sato R et al. A major influence of CYP2C19 genotype on the steady-state concentration of N-desmethylclobazam. Brain Dev 2004; 26: 530-534

[662] Koster M, Grohmann R, Engel RR et al. Seizures during antidepressant treatment in psychiatric inpatients-results from the transnational pharmacovigilance project "Arzneimittelsicherheit in der Psychiatrie" (AMSP) 1993-2008. Psychopharmacology (Berl) 2013; 230: 191-201

[663] Kotlyar M, Brauer LH, Tracy TS et al. Inhibition of CYP2D6 activity by bupropion. J Clin Psychopharmacol 2005; 25: 226-229

[664] Koyama E, Chiba K, Tani M et al. Identification of human cytochrome P450 isoforms involved in the stereoselective metabolism of mianserin enantiomers. J Pharmacol Exp Ther 1996; 278: 21-30

[665] Kozer E, Parvez S, Minassian BA et al. How high can we go with phenytoin? Ther Drug Monit 2002; 24: 386-389

[666] Kreuzer P, Landgrebe M, Wittmann M et al. Hypothermia associated with antipsychotic drug use: A clinical case series and review of current literature. J Clin Pharmacol 2012; 52: 1090-1097

[667] Krishna G, Moton A, Ma L et al. Effects of oral posaconazole on the pharmacokinetic properties of oral and intravenous midazolam: A phase I, randomized, open-label, crossover study in healthy volunteers. Clin Ther 2009; 31: 286-298

[668] Krishnan S, Moncrief S. An evaluation of the cytochrome p450 inhibition potential of lisdexamfetamine in human liver microsomes. Drug Metab Dispos 2007; 35: 180-184
[669] Krska J, Corner DA. Serum drug level monitoring in affective disorders. J Clin Pharm Ther 1992; 17: 357-363

[670] Kruithof MK, Bruins NA, van Roon EN. Coma after overdose with duloxetine. Ann Pharmacother 2011; 45: e5

[671] Kugelberg FC, Druid H, Carlsson B et al. Postmortem redistribution of the enantiomers of citalopram and its metabolites: An experimental study in rats. J Anal Toxicol 2004; 28: 631-637

[672] Kuhlman JJ Jr., Levine B, Johnson RE et al. Relationship of plasma buprenorphine and norbuprenorphine to withdrawal symptoms during dose induction, maintenance and withdrawal from sublingual buprenorphine. Addiction 1998; 93: 549-559

[673] Kurtz DL, Bergstrom RF, Goldberg MJ et al. The effect of sertraline on the pharmacokinetics of desipramine and imipramine. Clin Pharmacol Ther 1997; 62: 145-156

[674] Kuss HJ, Feistenauer E. Quantitative high-performance liquid chromatographic assay for the determination of maprotiline and oxaprotiline in human plasma. J Chromatogr 1981; 204: 349-353

[675] Kvist EE, Al-Shurbaji A, Dahl ML et al. Quantitative pharmacogenetics of nortriptyline: a novel approach. Clin Pharmacokinet 2001; 40: 869-877

[676] Kyhl LE, Li S, Faerch KU et al. Population pharmacokinetics of nalmefene in healthy subjects and its relation to mu-opioid receptor occupancy. Br J Clin Pharmacol 2016; 81: 290-300

[677] Labellarte M, Biederman J, Emslie G et al. Multiple-dose pharmacokinetics of fluvoxamine in children and adolescents. J Am Acad Child Adolesc Psychiatry 2004; 43: 1497-1505

[678] Laib AK, Brunen S, Pfeifer P et al. Serum concentrations of hydroxybupropion for dose optimization of depressed patients treated with bupropion. Ther Drug Monit 2014; 36: 473-479

[679] Laje G. Pharmacogenetics of mood disorders: What clinicians need to know. CNS Spectr 2013; 18: 272-284

[680] Laje G, McMahon F]. Genome-wide association studies of antidepressant outcome: A brief review. Prog Neuropsychopharmacol Biol Psychiatry 2011; 35: 1553-1557

[681] Landmark CJ, Johannessen SI, Tomson T. Dosing strategies for antiepileptic drugs: From a standard dose for all to individualised treatment by implementation of therapeutic drug monitoring. Epileptic Disord 2016; 18: 367-383

[682] Langel K, Engblom C, Pehrsson A et al. Drug testing in oral fluid-evaluation of sample collection devices. J Anal Toxicol 2008; 32: 393-401

[683] Langel K, Gjerde H, Favretto D et al. Comparison of drug concentrations between whole blood and oral fluid. Drug Test Anal 2014; 6: 461-471

[684] Lappenberg-Pelzer M. Identification and determination of opipramol metabolites in plasma and urine. J Anal Toxicol 1998; 22: 215-219

[685] Larson ME, Richards TM. Quantification of a methadone metabolite (EDDP) in urine: Assessment of compliance. Clin Med Res 2009; 7: 134-141

[686] Launonen E, Wallace I, Kotovirta E et al. Factors associated with non-adherence and misuse of opioid maintenance treatment medications and intoxicating drugs among Finnish maintenance treatment patients. Drug Alcohol Depend 2016; 162: 227-235

[687] Lautala P, Ethell BT, Taskinen J et al. The specificity of glucuronidation of entacapone and tolcapone by recombinant human UDPglucuronosyltransferases. Drug Metab Dispos 2000; 28 : 1385-1389

[688] Le Bloc'h Y, Woggon B, Weissenrieder H et al. Routine therapeutic drug monitoring in patients treated with $10-360 \mathrm{mg} /$ day citalopram. Ther Drug Monit 2003; 25: 600-608 
[689] Leavitt SB, Shinderman M, Maxwell S et al. When "enough" is not enough: new perspectives on optimal methadone maintenance dose. Mt Sinai J Med 2000; 67: 404-411

[690] Lee SY, Kim YG, Kim HG et al. Pharmacokinetic parameters of bromperidol in Korean subjects. Hum Psychopharmacol 2006; 21: 409-412

[691] Lefevre G, Buche M, Sedek G et al. Similar rivastigmine pharmacokinetics and pharmacodynamics in Japanese and white healthy participants following the application of novel rivastigmine patch. J Clin Pharmacol 2009; 49: 430-443

[692] Lemma GL, Wang Z, Hamman MA et al. The effect of short- and long-term administration of verapamil on the disposition of cytochrome P450 3A and P-glycoprotein substrates. Clin Pharmacol Ther 2006; 79: 218-230

[693] Leon J, Susce MT, Pan RM et al. A study of genetic (CYP2D6 and $A B C B 1$ ) and environmental (drug inhibitors and inducers) variables that may influence plasma risperidone levels. Pharmacopsychiatry 2007; 40: 93-102

[694] Lerer B, Segman RH, Tan EC et al. Combined analysis of 635 patients confirms an age-related association of the serotonin $2 \mathrm{~A}$ receptor gene with tardive dyskinesia and specificity for the non-orofacial subtype. Int J Neuropsychopharmacol 2005; 8: 411-425

[695] Lertxundi U, Domingo-Echaburu S, Hernandez R et al. Expert-based drug lists to measure anticholinergic burden: similar names, different results. Psychogeriatrics 2013; 13: 17-24

[696] Leucht S, Busch R, Kissling W et al. Early prediction of antipsychotic nonresponse among patients with schizophrenia. J Clin Psychiatry 2007; 68: 352-360

[697] Leucht S, Steimer W, Kreuz S et al. Doxepin plasma concentrations: is there really a therapeutic range? J Clin Psychopharmacol 2001; 21: 432-439

[698] Levine M, Truitt CA, O'Connor AD. Cardiotoxicity and serotonin syndrome complicating a milnacipran overdose. J Med Toxicol 2011; 7: 312-316

[699] Levran O, Peles E, Hamon S et al. CYP2B6 SNPs are associated with methadone dose required for effective treatment of opioid addiction. Addict Biol 2013; 18: 709-716

[700] Levran O, Peles E, Randesi M et al. Association of genetic variation in pharmacodynamic factors with methadone dose required for effective treatment of opioid addiction. Pharmacogenomics 2013; 14: 755-768

[701] LeWitt PA, Jennings D, Lyons KE et al. Pharmacokinetic-pharmacodynamic crossover comparison of two levodopa extension strategies. Mov Disord 2009; 24: 1319-1324

[702] Li-Bo D, Rong-Hua Z, Huan-De L et al. Quantitative analysis of trazodone in human plasma by using HPLC-fluorescence detector coupled with strong cation exchange chromatographic column: application to a pharmacokinetic study in Chinese healthy volunteers. J Chromatogr B Analyt Technol Biomed Life Sci 2014; 944: $43-48$

[703] Li AN, Dong F, He JL et al. The elimination rate after clozapine overdose in chinese schizophrenia patients: A population pharmacokinetics model study. Pharmacopsychiatry 2015; 48: 150-155

[704] Li F, Chin C, Wangsa J et al. Excretion and metabolism of milnacipran in humans after oral administration of milnacipran hydrochloride. Drug Metab Dispos 2012; 40: 1723-1735

[705] Li Q, Su YA, Liu Y et al. Pharmacokinetics and tolerability of extended-release quetiapine fumarate in Han Chinese patients with schizophrenia. Clin Pharmacokinet 2014; 53: 455-465

[706] Li RR, Sheng XY, Ma LY et al. Saliva and plasma monohydroxycarbamazepine concentrations in pediatric patients with epilepsy. Ther Drug Monit 2016; 38: 365-370
[707] Licht RW, Vestergaard P, Kessing LV et al. Psychopharmacological treatment with lithium and antiepileptic drugs: Suggested guidelines from the Danish Psychiatric Association and the Child and Adolescent Psychiatric Association in Denmark. Acta Psychiatr Scand Suppl 2003; $1-22$

[708] Lieber CS. The discovery of the microsomal ethanol oxidizing system and its physiologic and pathologic role. Drug Metab Rev 2004; 36: 511-529

[709] Lieberman JA, Stroup TS, McEvoy JP et al. Effectiveness of antipsychotic drugs in patients with chronic schizophrenia. N Engl J Med 2005; 353: 1209-1223

[710] Liechti ME, Quednow BB, Liakoni E et al. Pharmacokinetics and pharmacodynamics of gamma-hydroxybutyrate in healthy subjects. Br J Clin Pharmacol 2016; 81: 980-988

[711] Lin SK, Chen CK, Liu YL. Aripiprazole and dehydroaripiprazole plasma concentrations and clinical responses in patients with schizophrenia. J Clin Psychopharmacol 2011; 31: 758-762

[712] Lind AB, Reis M, Bengtsson F et al. Steady-state concentrations of mirtazapine, $\mathrm{N}$-desmethylmirtazapine, 8-hydroxymirtazapine and their enantiomers in relation to cytochrome P450 2D6 genotype, age and smoking behaviour. Clin Pharmacokinet 2009; 48: 63-70

[713] Lindberger M, Luhr O, Johannessen SI et al. Serum concentrations and effects of gabapentin and vigabatrin: Observations from a dose titration study. Ther Drug Monit 2003; 25: 457-462

[714] Lindenmayer JP. Long-acting injectable antipsychotics: Focus on olanzapine pamoate. Neuropsychiatr Dis Treat 2010; 6: 261-267

[715] Linder MW, Keck PE Jr.. Standards of laboratory practice: Antidepressant drug monitoring. National Academy of Clinical Biochemistry. Clin Chem 1998; 44: 1073-1084

[716] Lingam R, Scott J. Treatment non-adherence in affective disorders. Acta Psychiatr Scand 2002; 105: 164-172

[717] Lingjaerde O, Ahlfors UG, Bech P et al. The UKU side effect rating scale. A new comprehensive rating scale for psychotropic drugs and a cross-sectional study of side effects in neuroleptic-treated patients. Acta Psychiatr Scand Suppl 1987; 334: 1-100

[718] Linnet K, Ejsing TB. A review on the impact of P-glycoprotein on the penetration of drugs into the brain. Focus on psychotropic drugs. Eur Neuropsychopharmacol 2008; 18: 157-169

[719] Lintzeris N, Leung SY, Dunlop AJ et al. A randomised controlled trial of sublingual buprenorphine-naloxone film versus tablets in the management of opioid dependence. Drug Alcohol Depend 2013; 131: 119-126

[720] Liu MY, Meng SN, Wu HZ et al. Pharmacokinetics of single-dose and multiple-dose memantine in healthy chinese volunteers using an analytic method of liquid chromatography-tandem mass spectrometry. Clin Ther 2008; 30: 641-653

[721] Liu X, Lu YF, Guan X et al. Characterizing novel metabolic pathways of melatonin receptor agonist agomelatine using metabolomic approaches. Biochem Pharmacol 2016; 109: 70-82

[722] Liu Y, jiao ], Zhang C et al. A simplified method to determine five cytochrome p450 probe drugs by HPLC in a single run. Biol Pharm Bull 2009; 32: 717-720

[723] Liu ZQ, Shu Y, Huang SL et al. Effects of CYP2C19 genotype and CYP2C9 on fluoxetine $\mathrm{N}$-demethylation in human liver microsomes. Acta Pharmacol Sin 2001; 22: 85-90

[724] Livezey MR, Briggs ED, Bolles AK et al. Metoclopramide is metabolized by CYP2D6 and is a reversible inhibitor, but not inactivator, of CYP2D6. Xenobiotica 2014; 44: 309-319

[725] Loayza N, Crettol S, Riquier F et al. Adherence to antidepressant treatment: what the doctor thinks and what the patient says. Pharmacopsychiatry 2012; 45: 204-207 
[726] Lobo ED, Bergstrom RF, Reddy S et al. In vitro and in vivo evaluations of cytochrome P450 1A2 interactions with duloxetine. Clin Pharmacokinet 2008; 47: 191-202

[727] Lobo ED, Quinlan T, O'Brien L et al. Population pharmacokinetics of orally administered duloxetine in patients: implications for dosing recommendation. Clin Pharmacokinet 2009; 48: 189-197

[728] Locatelli I, Kastelic M, Koprivsek J et al. A population pharmacokinetic evaluation of the influence of CYP2D6 genotype on risperidone metabolism in patients with acute episode of schizophrenia. Eur J Pharm Sci 2010; 41: 289-298

[729] Lopez LV, Kane JM. Plasma levels of second-generation antipsychotics and clinical response in acute psychosis: A review of the literature. Schizophr Res 2013; 147: 368-374

[730] Loscher W, Luna-Tortos C, Romermann K et al. Do ATP-binding cassette transporters cause pharmacoresistance in epilepsy? Problems and approaches in determining which antiepileptic drugs are affected. Curr Pharm Des 2011; 17: 2808-2828

[731] Lotrich FE, Pollock BG. Aging and clinical pharmacology: implications for antidepressants. J Clin Pharmacol 2005; 45: 1106-1122

[732] Lucek R, Dixon R. Chlordiazepoxide concentrations in saliva and plasma measured by radioimmunoassay. Res Commun Chem Pathol Pharmacol 1980; 27: 397-400

[733] Lundberg J, Christophersen JS, Petersen KB et al. PET measurement of serotonin transporter occupancy: A comparison of escitalopram and citalopram. Int J Neuropsychopharmacol 2007; 10: 777-785

[734] Lundmark J, Bengtsson F, Nordin C et al. Therapeutic drug monitoring of selective serotonin reuptake inhibitors influences clinical dosing strategies and reduces drug costs in depressed elderly patients. Acta Psychiatr Scand 2000; 101: 354-359

[735] Lunell E, Curvall M. Nicotine delivery and subjective effects of Swedish portion snus compared with $4 \mathrm{mg}$ nicotine polacrilex chewing gum. Nicotine Tob Res 2011; 13: 573-578

[736] Luo JP, Vashishtha SC, Hawes EM et al. In vitro identification of the human cytochrome $\mathrm{p} 450$ enzymes involved in the oxidative metabolism of loxapine. Biopharm Drug Dispos 2011; 32: 398-407

[737] Luthi G, Blangy V, Eap CB et al. Buprenorphine and norbuprenorphine quantification in human plasma by simple protein precipitation and ultra-high performance liquid chromatography tandem mass spectrometry. J Pharm Biomed Anal 2013; 77: 1-8

[738] Luurila H, Olkkola KT. Pharmacokinetic-pharmacodynamic modelling of zopiclone effects on human central nervous system. Pharmacol Toxicol 1996; 78: 348-353

[739] Lynch M], Woods ], George N et al. Fatality due to amisulpride toxicity: a case report. Med Sci Law 2008; 48: 173-177

[740] Machino A, Jitsuiki H, Okamoto $Y$ et al. The valproate serum level in maintenance therapy for bipolar disorder in Japan. Hiroshima J Med Sci 2013; 62: 7-12

[741] Mackey TK, Liang BA. Improving global health governance to combat counterfeit medicines: A proposal for a UNODC-WHO-Interpol trilateral mechanism. BMC Med 2013; 11: 233

[742] Madan A, Graham RA, Carroll KM et al. Effects of prototypical microsomal enzyme inducers on cytochrome P450 expression in cultured human hepatocytes. Drug Metab Dispos 2003; 31: 421-431

[743] Madan A, Parkinson A, Faiman MD. Identification of the human P-450 enzymes responsible for the sulfoxidation and thiono-oxidation of diethyldithiocarbamate methyl ester: Role of P-450 enzymes in disulfiram bioactivation. Alcohol Clin Exp Res 1998; 22: 1212-1219

[744] Madsen H, Nielsen KK, Brosen K. Imipramine metabolism in relation to the sparteine and mephenytoin oxidation polymorphisms - a population study. Br J Clin Pharmacol 1995; 39: 433-439

[745] Maguire KP, Burrows GD, Norman TR et al. Metabolism and pharmacokinetics of dothiepin. Br J Clin Pharmacol 1981; 12: 405-409
[746] Maguire KP, Norman TR, Burrows GD et al. An evaluation of maprotiline intravenous kinetics and comparison of two oral doses. Eur J Clin Pharmacol 1980; 18: 249-254

[747] Magura S, Laudet AB, Mahmood D et al. Adherence to medication regimens and participation in dual-focus self-help groups. Psychiatr Serv 2002; 53: 310-316

[748] Mahmood I, Sahajwalla C. Clinical pharmacokinetics and pharmacodynamics of buspirone, an anxiolytic drug. Clin Pharmacokinet 1999; 36: $277-287$

[749] Majumdar AK, McCrea JB, Panebianco DL et al. Effects of aprepitant on cytochrome P450 3A4 activity using midazolam as a probe. Clin Pharmacol Ther 2003; 74: 150-156

[750] Mallaysamy S, Johnson MG, Rao PG et al. Population pharmacokinetics of lamotrigine in Indian epileptic patients. Eur J Clin Pharmacol 2013; 69: 43-52

[751] Mallikaarjun S, Kane JM, Bricmont P et al. Pharmacokinetics, tolerability and safety of aripiprazole once-monthly in adult schizophrenia: An open-label, parallel-arm, multiple-dose study. Schizophr Res 2013; 150: 281-288

[752] Mallikaarjun S, Salazar DE, Bramer SL. Pharmacokinetics, tolerability, and safety of aripiprazole following multiple oral dosing in normal healthy volunteers. J Clin Pharmacol 2004; 44: 179-187

[753] Mallinger AG, Himmelhoch JM, Thase ME et al. Plasma tranylcypromine: relationship to pharmacokinetic variables and clinical antidepressant actions. J Clin Psychopharmacol 1990; 10: 176-183

[754] Mamo D, Kapur S, Keshavan M et al. D2 receptor occupancy of olanzapine pamoate depot using positron emission tomography: An open-label study in patients with schizophrenia. Neuropsychopharmacology 2008; 33: 298-304

[755] Mamo D, Kapur S, Shammi CM et al. A PET study of dopamine D2 and serotonin 5-HT2 receptor occupancy in patients with schizophrenia treated with therapeutic doses of ziprasidone. Am J Psychiatry 2004; 161: 818-825

[756] Mandrioli R, Mercolini L, Saracino MA et al. Selective serotonin reuptake inhibitors (SSRIs): Therapeutic drug monitoring and pharmacological interactions. Curr Med Chem 2012; 19: 1846-1863

[757] Mann K, Hiemke C, Lotz J et al. Appropriateness of plasma level determinations for lithium and valproate in routine care of psychiatric inpatients with affective disorders. J Clin Psychopharmacol 2006; 26: 671-673

[758] Mann K, Hiemke C, Schmidt LG et al. Appropriateness of therapeutic drug monitoring for antidepressants in routine psychiatric inpatient care. Ther Drug Monit 2006; 28: 83-88

[759] Mannheimer B, Haslemo T, Lindh JD et al. Risperidone and venlafaxine metabolic ratios strongly predict a cyp2d6 poor metabolizing genotype. Ther Drug Monit 2016; 38: 127-134

[760] Mannheimer B, Holm J, Koukel L et al. Risperidone metabolic ratio as a biomarker of individual CYP2D6 genotype in schizophrenic patients. Eur J Clin Pharmacol 2014; 70: 695-699

[761] Mao J, Johnson TR, Shen Z et al. Prediction of crizotinib-midazolam interaction using the Simcyp population-based simulator: comparison of CYP3A time-dependent inhibition between human liver microsomes versus hepatocytes. Drug Metab Dispos 2013; 41: 343-352

[762] Marazziti D, Baroni S, Picchetti M et al. Pharmacokinetics and pharmacodynamics of psychotropic drugs: Effect of sex. CNS Spectr 2013; 18: 118-127

[763] Marcus SC, Olfson M, Pincus HA et al. Therapeutic drug monitoring of mood stabilizers in Medicaid patients with bipolar disorder. Am J Psychiatry 1999; 156: 1014-1018

[764] Markert C, Ngui P, Hellwig R et al. Influence of St. John's wort on the steady-state pharmacokinetics and metabolism of bosentan. Int J Clin Pharmacol Ther 2014; 52: 328-336 
[765] Markoula S, Teotonio R, Ratnaraj N et al. Lacosamide serum concentrations in adult patients with epilepsy: The influence of gender, age, dose, and concomitant antiepileptic drugs. Ther Drug Monit 2014; 36: 494-498

[766] Markowitz JS, Patrick KS. Differential pharmacokinetics and pharmacodynamics of methylphenidate enantiomers: Does chirality matter? J Clin Psychopharmacol 2008; 28: S54-S61

[767] Martin P, Dirks B, Gertsik L et al. Safety and pharmacokinetics of lisdexamfetamine dimesylate in adults with clinically stable schizophrenia: A randomized, double-blind, placebo-controlled trial of ascending multiple doses. J Clin Psychopharmacol 2014; 34 682-689

[768] Martines C, Gatti G, Sasso E et al. The disposition of primidone in elderly patients. Br J Clin Pharmacol 1990; 30: 607-611

[769] Martinez C, Albet C, Agundez JA et al. Comparative in vitro and in vivo inhibition of cytochrome P450 CYP1A2, CYP2D6, and CYP3A by H2-receptor antagonists. Clin Pharmacol Ther 1999; 65: 369-376

[770] Mas S, Gasso P, Ritter MA et al. Pharmacogenetic predictor of extrapyramidal symptoms induced by antipsychotics: multilocus interaction in the mTOR pathway. Eur Neuropsychopharmacol 2015: 25: $51-59$

[771] Mason BJ, Goodman AM, Dixon RM et al. A pharmacokinetic and pharmacodynamic drug interaction study of acamprosate and naltrexone. Neuropsychopharmacology 2002; 27: 596-606

[772] Masson CL, Rainey PM, Moody DE et al. Effects of HCV seropositive status on buprenorphine pharmacokinetics in opioid-dependent individuals. Am J Addict 2014; 23: 34-40

[773] Matsui DM. Therapeutic drug monitoring in pregnancy. Ther Drug Monit 2012; 34: 507-51

[774] Matthaei J, Brockmoller J, Tzvetkov MV et al. Heritability of metoprolol and torsemide pharmacokinetics. Clin Pharmacol Ther 2015; 98: 611-621

[775] Mattila MA, Larni HM. Flunitrazepam: A review of its pharmacological properties and therapeutic use. Drugs 1980; 20: 353-374

[776] Maurer HH, Sauer C, Theobald DS. Toxicokinetics of drugs of abuse: current knowledge of the isoenzymes involved in the human metabolism of tetrahydrocannabinol, cocaine, heroin, morphine, and codeine. Ther Drug Monit 2006; 28: 447-453

[777] Mauri MC, Laini V, Boscati L et al. Long-term treatment of chronic schizophrenia with risperidone: A study with plasma levels. Eur Psychiatry 2001; 16: 57-63

[778] Mauri MC, Maffini M, Di Pace C et al. "Long-acting" olanzapine in maintenance therapy of schizophrenia: A study with plasma levels. Int J Psychiatry Clin Pract 2015; 19: 99-105

[779] Mauri MC, Paletta S, Maffini M et al. Clinical pharmacology of atypical antipsychotics: An update. EXCLI ] 2014; 13: 1163-1191

[780] Mauri MC, Steinhilber CP, Marino R et al. Clinical outcome and olanzapine plasma levels in acute schizophrenia. Eur Psychiatry 2005; 20: 55-60

[781] Mauri MC, Volonteri LS, Colasanti A et al. Clinical pharmacokinetics of atypical antipsychotics: A critical review of the relationship between plasma concentrations and clinical response. Clin Pharmacokinet 2007; 46: 359-388

[782] May TW, Korn-Merker E, Rambeck B. Clinical pharmacokinetics of oxcarbazepine. Clin Pharmacokinet 2003; 42: 1023-1042

[783] May TW, Korn-Merker E, Rambeck B et al. Pharmacokinetics of sulthiame in epileptic patients. Ther Drug Monit 1994; 16: 251-257

[784] May TW, Rambeck B, Jurgens U. Serum concentrations of topiramate in patients with epilepsy: Influence of dose, age, and comedication. Ther Drug Monit 2002; 24: 366-374
[785] May TW, Rambeck B, Neb R et al. Serum concentrations of pregabalin in patients with epilepsy: The influence of dose, age, and comedication. Ther Drug Monit 2007; 29: 789-794

[786] Mayo BC, Biggs SR, Chasseaud LF et al. The metabolic fate of Sormodren (bornaprine hydrochloride) in animals and humans. Xenobiotica 1980; 10: 873-888

[787] Mazur A, Lichti CF, Prather PL et al. Characterization of human hepatic and extrahepatic UDP-glucuronosyltransferase enzymes involved in the metabolism of classic cannabinoids. Drug Metab Dispos 2009; 37: 1496-1504

[788] McAlpine DE, Biernacka JM, Mrazek DA et al. Effect of cytochrome P450 enzyme polymorphisms on pharmacokinetics of venlafaxine. Ther Drug Monit 2011; 33: 14-20

[789] McCarthy M], Leckband SG, Kelsoe JR. Pharmacogenetics of lithium response in bipolar disorder. Pharmacogenomics 2010; 11: 1439-1465

[790] McDonald MG, Au NT, Rettie AE. P450-Based drug-drug interactions of amiodarone and its metabolites: Diversity of inhibitory mechanisms. Drug Metab Dispos 2015; 43: 1661-1669

[791] Mclntyre IM, Mallett P, Stabley R. Postmortem distribution of trazodone concentrations. Forensic Sci Int 2015; 251: 195-201

[792] McKenzie ME, Roswell-Harris D. A controlled trial of prothipendyl (tolnate) in mentally subnormal patients. Br J Psychiatry 1966; 112: 95-100

[793] Medori R, Mannaert E, Gründer G. Plasma antipsychotic concentration and receptor occupancy, with special focus on risperidone long-acting injectable. Eur Neuropsychopharmacol 2006; 16: 233-240

[794] Mehler-Wex C, Kolch M, Kirchheiner J et al. Drug monitoring in child and adolescent psychiatry for improved efficacy and safety of psychopharmacotherapy. Child Adolesc Psychiatry Ment Health 2009; 3: 14

[795] Meijer WE, Bouvy ML, Heerdink ER et al. Spontaneous lapses in dosing during chronic treatment with selective serotonin reuptake inhibitors. Br J Psychiatry 2001; 179: 519-522

[796] Meineke I, Kress I, Poser W et al. Therapeutic drug monitoring of mirtazapine and its metabolite desmethylmirtazapine by HPLC with fluorescence detection. Ther Drug Monit 2004; 26: 277-283

[797] Meisenzahl EM, Schmitt G, Gründer G et al. Striatal D2/D3 receptor occupancy, clinical response and side effects with amisulpride: An iodine-123-iodobenzamide SPET study. Pharmacopsychiatry 2008; 41: $169-175$

[798] Menke A, Klengel T, Binder EB. Epigenetics, depression and antidepressant treatment. Curr Pharm Des 2012; 18: 5879-5889

[799] Meyer-Barner M, Meineke I, Schreeb KH et al. Pharmacokinetics of doxepin and desmethyldoxepin: An evaluation with the population approach. Eur J Clin Pharmacol 2002; 58: 253-257

[800] Meyer JH. Imaging the serotonin transporter during major depressive disorder and antidepressant treatment. J Psychiatry Neurosci 2007; 32: $86-102$

[801] Meyer JH, Wilson AA, Sagrati S et al. Serotonin transporter occupancy of five selective serotonin reuptake inhibitors at different doses: An [11C]DASB positron emission tomography study. Am J Psychiatry 2004; 161: 826-835

[802] Meyer MR, Schutz A, Maurer HH. Contribution of human esterases to the metabolism of selected drugs of abuse. Toxicol Lett 2015; 232 159-166

[803] Meyer RP, Gehlhaus M, Knoth R et al. Expression and function of cytochrome p450 in brain drug metabolism. Curr Drug Metab 2007; 8: 297-306

[804] Michaud V, Mouksassi MS, Labbe L et al. Inhibitory effects of propafenone on the pharmacokinetics of caffeine in humans. Ther Drug Monit 2006; 28: 779-783 
[805] Michelson D, Read HA, Ruff DD et al. CYP2D6 and clinical response to atomoxetine in children and adolescents with ADHD. J Am Acad Child Adolesc Psychiatry 2007; 46: 242-251

[806] Mihara K, Otani K, Suzuki A et al. Relationship between the CYP2D6 genotype and the steady-state plasma concentrations of trazodone and its active metabolite m-chlorophenylpiperazine. Psychopharmacology (Berl) 1997; 133: 95-98

[807] Mihara K, Yasui-Furukori N, Kondo T et al. Relationship between plasma concentrations of trazodone and its active metabolite, $\mathrm{m}$-chlorophenylpiperazine, and its clinical effect in depressed patients. Ther Drug Monit 2002; 24: 563-566

[808] Mikami A, Ohtani H, Hori S et al. Pharmacokinetic model incorporating mechanism-based inactivation of CYP2D6 can explain both non-linear kinetics and drug interactions of paroxetine. Int J Clin Pharmacol Ther 2013; 51: 374-382

[809] Miller DS. Regulation of ABC transporters blood-brain barrier: The good, the bad, and the ugly. Adv Cancer Res 2015; 125: 43-70

[810] Milosheska D, Grabnar I, Vovk T. Dried blood spots for monitoring and individualization of antiepileptic drug treatment. Eur J Pharm Sci 2015; 75: 25-39

[811] Mimaki T. Clinical pharmacology and therapeutic drug monitoring of zonisamide. Ther Drug Monit 1998; 20: 593-597

[812] Mitchell TB, White JM, Somogyi AA et al. Comparative pharmacodynamics and pharmacokinetics of methadone and slow-release oral morphine for maintenance treatment of opioid dependence. Drug Alcohol Depend 2003; 72: 85-94

[813] Miura H. Zonisamide monotherapy with once-daily dosing in children with cryptogenic localization-related epilepsies: Clinical effects and pharmacokinetic studies. Seizure 2004; 13 Suppl 1: S17-S23 discussion S24-15

[814] Miura M, Ohkubo T. Identification of human cytochrome P450 enzymes involved in the major metabolic pathway of fluvoxamine. Xenobiotica 2007; 37: 169-179

[815] Molden E, Lunde $\mathrm{H}$, Lunder $\mathrm{N}$ et al. Pharmacokinetic variability of aripiprazole and the active metabolite dehydroaripiprazole in psychiatric patients. Ther Drug Monit 2006; 28: 744-749

[816] Montgomery S, McAuley R, Montgomery DB. Relationship between mianserin plasma levels and antidepressant effect in a double-blind trial comparing a single night-time and divided daily dose regimens. $\mathrm{Br}$ J Clin Pharmacol 1978; 5: (Suppl 1): 71S-76S

[817] Moody DE, Chang Y, Huang $W$ et al. The in vivo response of novel buprenorphine metabolites, M1 and M3, to antiretroviral inducers and inhibitors of buprenorphine metabolism. Basic Clin Pharmaco Toxicol 2009; 105: 211-215

[818] Morgan DD, Robinson JD, Mendenhall CL. Clinical pharmacokinetics of chlordiazepoxide in patients with alcoholic hepatitis. Eur J Clin Pharmacol 1981; 19: 279-285

[819] Morris RG, Black AB, Harris AL et al. Lamotrigine and therapeutic drug monitoring: Retrospective survey following the introduction of a routine service. $\mathrm{Br}$ J Clin Pharmacol 1998; 46: 547-551

[820] Morris RG, Lee MY, Cleanthous X et al. Long-term follow-up using a higher target range for lamotrigine monitoring. Ther Drug Monit 2004; 26: 626-632

[821] Morselli PL, Baruzzi A, Gerna M et al. Carbamazepine and carbamazepine-10, 11-epoxide concentrations in human brain. $\mathrm{Br}$ J Clin Pharmacol 1977; 4: 535-540

[822] Mossner R, Schuhmacher A, Kuhn KU et al. Functional serotonin 1A receptor variant influences treatment response to atypical antipsychotics in schizophrenia. Pharmacogenet Genomics 2009; 19: 91-94

[823] Mrazek DA. Psychiatric pharmacogenomic testing in clinical practice. Dialogues Clin Neurosci 2010; 12: 69-76

[824] Müller-Isberner R, Freese R, Jockel D et al. Forensic psychiatric assessment and treatment in Germany. Legal framework, recent developments, and current practice. Int J Law Psychiatry 2000; 23: 467-480

[825] Müller H, Eusterschulte B, Havemann-Reinecke U et al. Forensische Aspekte des therapeutischen Drug-Monitorings (TDM) in der Psychiatrie. Psychopharmakotherapie 2009; 16: 52-56

[826] Müller MJ, Dragicevic A, Fric M et al. Therapeutic drug monitoring of tricyclic antidepressants: How does it work under clinical conditions? Pharmacopsychiatry 2003; 36: 98-104

[827] Müller MJ, Eich FX, Regenbogen B et al. Amisulpride doses and plasma levels in different age groups of patients with schizophrenia or schizoaffective disorder. J Psychopharmacol 2009; 23: 278-286

[828] Müller MJ, Härtter S, Kohler D et al. Serum levels of sulpiride enantiomers after oral treatment with racemic sulpiride in psychiatric patients: a pilot study. Pharmacopsychiatry 2001; 34: 27-32

[829] Müller M], Regenbogen B, Härtter S et al. Therapeutic drug monitoring for optimizing amisulpride therapy in patients with schizophrenia. J Psychiatr Res 2007; 41: 673-679

[830] Müller T. Pharmacokinetic considerations for the use of levodopa in the treatment of Parkinson disease: Focus on levodopa/carbidopa/ entacapone for treatment of levodopa-associated motor complications. Clin Neuropharmacol 2013; 36: 84-91

[831] Myung W, Jang JH, Yoon $\mathrm{H}$ et al. Therapeutic drug monitoring of mirtazapine in a routine outpatient setting in Asian psychiatric patients. Pharmacopsychiatry 2014; 47: 210-214

[832] Nagasaka Y, Oda K, Iwatsubo T et al. Effects of aripiprazole and its active metabolite dehydroaripiprazole on the activities of drug efflux transporters expressed both in the intestine and at the blood-brain barrier. Biopharm Drug Dispos 2012; 33: 304-315

[833] Nagy CF, Kumar D, Cullen El et al. Steady-state pharmacokinetics and safety of donepezil $\mathrm{HCl}$ in subjects with moderately impaired renal function. Br J Clin Pharmacol 2004; 58: (Suppl 1): 18-24

[834] Naik H, Chan S, Vakilynejad M et al. A population pharmacokineticpharmacodynamic meta-analysis of vortioxetine in patients with major depressive disorder. Basic Clin Pharmacol Toxicol 2016; 118 : 344-355

[835] Naito H, Wachi M, Nishida M. Clinical effects and plasma concentrations of long-term clonazepam monotherapy in previously untreated epileptics. Acta Neurol Scand 1987; 76: 58-63

[836] Nakajima S, Caravaggio F, Mamo DC et al. Dopamine $D(2) /(3)$ receptor availability in the striatum of antipsychotic-free older patients with schizophrenia-A [(1)(1)C]-raclopride PET study. Schizophr Res 2015; 164: 263-267

[837] Nakajima S, Uchida H, Bies RR et al. Dopamine D2/3 receptor occupancy following dose reduction is predictable with minimal plasma antipsychotic concentrations: An open-label clinical trial. Schizophr Bull 2016; 42: 212-219

[838] Nakamura A, Mihara K, Nagai G et al. Prediction of an optimal dose of lamotrigine for augmentation therapy in treatment-resistant depressive disorder from plasma lamotrigine concentration at week 2. Ther Drug Monit 2016; 38: 379-382

[839] Nakamura K, Yokoi T, Inoue K et al. CYP2D6 is the principal cytochrome $\mathrm{P} 450$ responsible for metabolism of the histamine $\mathrm{H} 1$ antagonist promethazine in human liver microsomes. Pharmacogenetics 1996; 6: 449-457

[840] Nakamura T, Kubota T, Iwakaji A et al. Clinical pharmacology study of cariprazine (MP-214) in patients with schizophrenia (12-week treatment). Drug Des Devel Ther 2016; 10: 327-338

[841] Narimatsu S, Kariya S, Isozaki S et al. Involvement of CYP2D6 in oxidative metabolism of cinnarizine and flunarizine in human liver microsomes. Biochem Biophys Res Commun 1993; 193: 1262-1268

[842] Nazirizadeh Y, Vogel F, Bader W et al. Serum concentrations of paliperidone versus risperidone and clinical effects. Eur J Clin Pharmacol 2010; 66: 797-803 
[843] Neels HM, Sierens AC, Naelaerts K et al. Therapeutic drug monitoring of old and newer anti-epileptic drugs. Clin Chem Lab Med 2004; 42: 1228-1255

[844] Nemoda Z, Angyal N, Tarnok Z et al. Carboxylesterase 1 gene polymorphism and methylphenidate response in ADHD. Neuropharmacology 2009; 57: 731-733

[845] Nesvag R, Hendset M, Refsum $\mathrm{H}$ et al. Serum concentrations of risperidone and $9-\mathrm{OH}$ risperidone following intramuscular injection of long-acting risperidone compared with oral risperidone medication. Acta Psychiatr Scand 2006; 114: 21-26

[846] Neuhaus W, Mandikova J, Pawlowitsch R et al. Blood-brain barrier in vitro models as tools in drug discovery: Assessment of the transport ranking of antihistaminic drugs. Pharmazie 2012; 67: 432-439

[847] Nguyen TT, Benech H, Pruvost A et al. A limited sampling strategy based on maximum a posteriori Bayesian estimation for a five-probe phenotyping cocktail. Eur J Clin Pharmacol 2016; 72: 39-51

[848] Nichols Al, Focht K, jiang Q et al. Pharmacokinetics of venlafaxine extended release $75 \mathrm{mg}$ and desvenlafaxine $50 \mathrm{mg}$ in healthy CYP2D6 extensive and poor metabolizers: A randomized, open-label, two-period, parallel-group, crossover study. Clin Drug Investig 2011; 31: $155-167$

[849] Nicolas JM, Collart P, Gerin B et al. In vitro evaluation of potential drug interactions with levetiracetam, a new antiepileptic agent. Drug Metab Dispos 1999; 27: 250-254

[850] Nikisch G, Baumann P, Oneda B et al. Cytochrome P450 and ABCB1 genetics: Association with quetiapine and norquetiapine plasma and cerebrospinal fluid concentrations and with clinical response in patients suffering from schizophrenia. A pilot study. J Psychopharmacol 2011; 25: 896-907

[851] Nikisch G, Baumann P, Wiedemann G et al. Quetiapine and norquetiapine in plasma and cerebrospinal fluid of schizophrenic patients treated with quetiapine: Correlations to clinical outcome and HVA, 5-HIAA, and MHPG in CSF. J Clin Psychopharmacol 2010; 30: $496-503$

[852] Nikisch G, Mathe AA, Czernik A et al. Stereoselective metabolism of citalopram in plasma and cerebrospinal fluid of depressive patients: Relationship with 5-HIAA in CSF and clinical response. J Clin Psychopharmacol 2004; 24: 283-290

[853] Nikolaus S, Larisch R, Beu M et al. In vivo measurement of D2 receptor density and affinity for $18 \mathrm{~F}-(3-\mathrm{N}$-methyl)benperidol in the rat striatum with a PET system for small laboratory animals. J Nucl Med 2003; 44: 618-624

[854] Nilausen DO, Zuiker RG, van Gerven J. The perception and pharmacokinetics of a $20-\mathrm{mg}$ dose of escitalopram orodispersible tablets in a relative bioavailability study in healthy men. Clin Ther 2011; 33 : 1492-1502

[855] Nilsson EB, Rimmele SM, Schmidt EK et al. Pharmacokinetics and relative bioavailability of opipramol in the new INSIDON-sustained release preparation. Fortschr Neurol Psychiatr 1998; 66: (Suppl 1): S13-S16

[856] Nilsson MI, Meresaar U, Anggard E. Clinical pharmacokinetics of methadone. Acta Anaesthesiol Scand Suppl 1982; 74: 66-69

[857] Nishikage $H$, Nakanishi $T$, Takamitsu $Y$ et al. Sequential changes in the plasma concentration of risperidone following intentional overdose. Clin Neuropharmacol 2002; 25: 307-309

[858] Nishikawa N, Nagai M, Moritoyo T et al. Plasma amantadine concentrations in patients with Parkinson's disease. Parkinsonism Relat Disord 2009; 15: 351-353

[859] Nishimura Y, Kurata N, Sakurai E et al. Inhibitory effect of antituberculosis drugs on human cytochrome P450-mediated activities. J Pharmacol Sci 2004; 96: 293-300

[860] Niwa T, Imagawa Y, Yamazaki H. Drug interactions between nine antifungal agents and drugs metabolized by human cytochromes P450. Curr Drug Metab 2014; 15: 651-679
[861] Nnadi CU, Malhotra AK. Clinical and pharmacogenetic studies of iloperidone. Per Med 2008; 5: 367-375

[862] Noetzli M, Guidi M, Ebbing K et al. Population pharmacokinetic study of memantine: Effects of clinical and genetic factors. Clin Pharmacokinet 2013; 52: 211-223

[863] Noetzli M, Guidi M, Ebbing K et al. Population pharmacokinetic approach to evaluate the effect of CYP2D6, CYP3A, ABCB1, POR and NR112 genotypes on donepezil clearance. Br J Clin Pharmacol 2014; 78: $135-144$

[864] Nogami T, Takano H, Arakawa R et al. Occupancy of serotonin and norepinephrine transporter by milnacipran in patients with major depressive disorder: A positron emission tomography study with [(11)C]DASB and (S,S)-[(18)F]FMeNER-D(2). Int J Neuropsychopharmacol 2013; 16: 937-943

[865] Norman T, Chiu E, James RH et al. Single oral dose pharmacokinetics of tiapride in patients with Huntington's disease. Eur J Clin Pharmacol 1987; 32: 583-586

[866] Nozawa M, Ohnuma T, Matsubara Y et al. The relationship between the response of clinical symptoms and plasma olanzapine concentration, based on pharmacogenetics: Juntendo University Schizophrenia Projects (JUSP). Ther Drug Monit 2008; 30: 35-40

[867] Nutt JG, Fellman JH. Pharmacokinetics of levodopa. Clin Neuropharmacol 1984; 7: 35-49

[868] Nyberg S, Nordstrom AL, Halldin C et al. Positron emission tomography studies on D2 dopamine receptor occupancy and plasma antipsychotic drug levels in man. Int Clin Psychopharmacol 1995; 10: (Suppl 3): 81-85

[869] Nyholm D. Pharmacokinetic optimisation in the treatment of Parkinson's disease: An update. Clin Pharmacokinet 2006; 45: 109-136

[870] Nyholm D, Ehrnebo M, Lewander T et al. Frequent administration of levodopa/carbidopa microtablets vs levodopa/carbidopa/entacapone in healthy volunteers. Acta Neurol Scand 2013; 127: 124-132

[871] Nyholm D, Odin P, Johansson A et al. Pharmacokinetics of levodopa, carbidopa, and 3-O-methyldopa following 16-hour jejunal infusion of levodopa-carbidopa intestinal gel in advanced Parkinson's disease patients. AAPS J 2013; 15: 316-323

[872] O'Connor S, Ayres A, Cortellini L et al. Process improvement methods increase the efficiency, accuracy, and utility of a neurocritical care research repository. Neurocrit Care 2012; 17: 90-96

[873] O’Dushlaine C, Ripke S, Ruderfer DM et al. Rare copy number variation in treatment-resistant major depressive disorder. Biol Psychiatry 2014; 76: 536-541

[874] O'Mahony D, Gallagher PF. Inappropriate prescribing in the older population: Need for new criteria. Age Ageing 2008; 37: 138-141

[875] O'Mahony D, O'Sullivan D, Byrne S et al. STOPP/START criteria for potentially inappropriate prescribing in older people: Version 2. Age Ageing 2015; 44: 213-218

[876] Obach RS, Cox LM, Tremaine LM. Sertraline is metabolized by multiple cytochrome P450 enzymes, monoamine oxidases, and glucuronyl transferases in human: An in vitro study. Drug Metab Dispos 2005; 33: 262-270

[877] Ochs HR, Greenblatt DJ, Friedman H et al. Bromazepam pharmacokinetics: Influence of age, gender, oral contraceptives, cimetidine, and propranolol. Clin Pharmacol Ther 1987; 41: 562-570

[878] Oda S, Fukami T, Yokoi T et al. A comprehensive review of UDP-glucuronosyltransferase and esterases for drug development. Drug Metab Pharmacokinet 2015; 30: 30-51

[879] Ogilvie BW, Yerino P, Kazmi F et al. The proton pump inhibitor, omeprazole, but not lansoprazole or pantoprazole, is a metabolismdependent inhibitor of CYP2C19: Implications for coadministration with clopidogrel. Drug Metab Dispos 2011; 39: 2020-2033 
[880] Ohman D, Cherma MD, Norlander B et al. Determination of serum reboxetine enantiomers in patients on chronic medication with racemic reboxetine. Ther Drug Monit 2003; 25: 174-182

[881] Ohman D, Norlander B, Peterson C et al. Bioanalysis of racemic reboxetine and its desethylated metabolite in a therapeutic drug monitoring setting using solid phase extraction and HPLC. Ther Drug Monit 2001; 23: 27-34

[882] Ohno Y, Hisaka A, Ueno M et al. General framework for the prediction of oral drug interactions caused by CYP3A4 induction from in vivo information. Clin Pharmacokinet 2008; 47: 669-680

[883] Okudaira T, Kotegawa T, Imai H et al. Effect of the treatment period with erythromycin on cytochrome P450 3A activity in humans. J Clin Pharmacol 2007; 47: 871-876

[884] Olesen OV, Linnet K. Contributions of five human cytochrome P450 isoforms to the $\mathrm{N}$-demethylation of clozapine in vitro at low and high concentrations. J Clin Pharmacol 2001; 41: 823-832

[885] Olesen OV, Linnet K. Hydroxylation and demethylation of the tricyclic antidepressant nortriptyline by cDNA-expressed human cytochrome P-450 isozymes. Drug Metab Dispos 1997; 25: 740-744

[886] Olesen OV, Linnet K. Identification of the human cytochrome P450 isoforms mediating in vitro $\mathrm{N}$-dealkylation of perphenazine. $\mathrm{Br} J \mathrm{Clin}$ Pharmacol 2000; 50: 563-571

[887] Ono S, Hatanaka T, Miyazawa S et al. Human liver microsomal diazepam metabolism using cDNA-expressed cytochrome P450s: role of CYP2B6, 2C19 and the 3A subfamily. Xenobiotica 1996; 26: $1155-1166$

[888] Orlando R, De Martin S, Andrighetto L et al. Fluvoxamine pharmacokinetics in healthy elderly subjects and elderly patients with chronic heart failure. Br J Clin Pharmacol 2010; 69: 279-286

[889] Orsulak PJ. Therapeutic monitoring of antidepressant drugs: current methodology and applications. J Clin Psychiatry 1986; 47 Suppl: 39-52

[890] Orsulak PJ. Therapeutic monitoring of antidepressant drugs: guidelines updated. Ther Drug Monit 1989; 11: 497-507

[891] Orsulak PJ, Schildkraut J]. Guidelines for therapeutic monitoring of tricyclic antidepressant plasma levels. Ther Drug Monit 1979; 1 : 199-208

[892] Ortiz de Montellano PR. Cytochrome P450-activated prodrugs. Future Med Chem 2013; 5: 213-228

[893] Osanai T, Ohkubo T, Yasui N et al. Effect of itraconazole on the pharmacokinetics and pharmacodynamics of a single oral dose of brotizolam. Br J Clin Pharmacol 2004; 58: 476-481

[894] Ostad Haji E, Mann K, Dragicevic A et al. Potential cost-effectiveness of therapeutic drug monitoring for depressed patients treated with citalopram. Ther Drug Monit 2013; 35: 396-401

[895] Ostad Haji E, Tadic A, Wagner S et al. Association between citalopram serum levels and clinical improvement of patients with major depression. J Clin Psychopharmacol 2011; 31: 281-286

[896] Ostad Haji E, Tadic A, Wagner S et al. Early improvement and serum concentrations of citalopram to predict antidepressant drug response of patients with major depression. Pharmacopsychiatry 2013; 46: 261-266

[897] Ostad Haji E, Wagner S, Fric M et al. Quetiapine and norquetiapine serum concentrations and clinical effects in depressed patients under augmentation therapy with quetiapine. Ther Drug Monit 2013; 35: 539-545

[898] Ota T, Shinotoh H, Fukushi K et al. Estimation of plasma IC50 of donepezil for cerebral acetylcholinesterase inhibition in patients with Alzheimer disease using positron emission tomography. Clin Neuropharmacol 2010; 33: 74-78

[899] Othman AA, Dutta S. Population pharmacokinetics of levodopa in subjects with advanced Parkinson's disease: Levodopa-carbidopa intestinal gel infusion vs. oral tablets. Br J Clin Pharmacol 2014; 78: 94-105

[900] Palego L, Biondi L, Giannaccini G et al. Clozapine, norclozapine plasma levels, their sum and ratio in 50 psychotic patients: Influence of patient-related variables. Prog Neuropsychopharmacol Biol Psychiatry 2002; 26: 473-480

[901] Palmer EC, Binns LN, Carey H. Levomilnacipran: A new serotoninnorepinephrine reuptake inhibitor for the treatment of major depressive disorder. Ann Pharmacother 2014; 48: 1030-1039

[902] Panagiotidis G, Arthur HW, Lindh JD et al. Depot haloperidol treatment in outpatients with schizophrenia on monotherapy: Impact of CYP2D6 polymorphism on pharmacokinetics and treatment outcome. Ther Drug Monit 2007; 29: 417-422

[903] Panchaud A, Weisskopf E, Winterfeld U et al. Pharmacokinetic alterations in pregnancy and use of therapeutic drug monitoring. Therapie 2014; 69: 223-234

[904] Paris BL, Ogilvie BW, Scheinkoenig JA et al. In vitro inhibition and induction of human liver cytochrome p450 enzymes by milnacipran. Drug Metab Dispos 2009; 37: 2045-2054

[905] Park JY, Kim KA, Park PW et al. Effect of CYP3A5 * 3 genotype on the pharmacokinetics and pharmacodynamics of alprazolam in healthy subjects. Clin Pharmacol Ther 2006; 79: 590-599

[906] Park PW, Seo YH, Ahn JY et al. Effect of CYP3A5 * 3 genotype on serum carbamazepine concentrations at steady-state in Korean epileptic patients. J Clin Pharm Ther 2009; 34: 569-574

[907] Parker DR, McIntyre IM. Case studies of postmortem quetiapine: therapeutic or toxic concentrations? J Anal Toxicol 2005; 29: 407-412

[908] Pasina L, Djade CD, Lucca U et al. Association of anticholinergic burden with cognitive and functional status in a cohort of hospitalized elderly: comparison of the anticholinergic cognitive burden scale and anticholinergic risk scale: Results from the REPOSI study. Drugs Aging 2013; 30: 103-112

[909] Patrick KS, Straughn AB, Minhinnett RR et al. Influence of ethanol and gender on methylphenidate pharmacokinetics and pharmacodynamics. Clin Pharmacol Ther 2007; 81: 346-353

[910] Patsalos PN. The clinical pharmacology profile of the new antiepileptic drug perampanel: A novel noncompetitive AMPA receptor antagonist. Epilepsia 2015; 56: 12-27

[911] Patsalos PN, Berry D]. Therapeutic drug monitoring of antiepileptic drugs by use of saliva. Ther Drug Monit 2013; 35: 4-29

[912] Patsalos PN, Berry DJ, Bourgeois BF et al. Antiepileptic drugs--best practice guidelines for therapeutic drug monitoring: A position paper by the subcommission on therapeutic drug monitoring, ILAE Commission on Therapeutic Strategies. Epilepsia 2008; 49: 1239-1276

[913] Patteet L, Cappelle D, Maudens KE et al. Advances in detection of antipsychotics in biological matrices. Clin Chim Acta 2015; 441: $11-22$

[914] Patteet L, Maudens KE, Morrens M et al. Determination of common antipsychotics in quantisal-collected oral fluid by UHPLC-MS/MS: Method validation and applicability for therapeutic drug monitoring. Ther Drug Monit 2016; 38: 87-97

[915] Patteet L, Maudens KE, Sabbe B et al. High throughput identification and quantification of 16 antipsychotics and 8 major metabolites in serum using ultra-high performance liquid chromatography-tandem mass spectrometry. Clin Chim Acta 2014; 429: 51-58

[916] Patteet L, Maudens KE, Stove CP et al. The use of dried blood spots for quantification of 15 antipsychotics and 7 metabolites with ultra-high performance liquid chromatography - tandem mass spectrometry. Drug Test Anal 2015; 7: 502-511

[917] Patteet L, Morrens M, Maudens KE et al. Therapeutic drug monitoring of common antipsychotics. Ther Drug Monit 2012; 34: 629-651 
[918] Paulzen M, Eap CB, Gründer G et al. Pharmacokinetic interaction between valproic acid, meropenem, and risperidone. J Clin Psychopharmacol 2016; 36: 90-92

[919] Paulzen M, Groppe S, Tauber SC et al. Venlafaxine and O-desmethylvenlafaxine concentrations in plasma and cerebrospinal fluid. J Clin Psychiatry 2015; 76: 25-31

[920] Paulzen M, Haen E, Stegmann B et al. Clinical response in a risperidone-medicated naturalistic sample: Patients' characteristics and dose-dependent pharmacokinetic patterns. Eur Arch Psychiatry Clin Neurosci. 2016;

[921] Paulzen M, Henkel K, Tauber S et al. Plasma levels and cerebrospinal fluid penetration of venlafaxine in a patient with a nonfatal overdose during a suicide attempt. J Clin Psychopharmacol 2014; 34 398-399

[922] Paz E, Bouzas L, Hermida J et al. Evaluation of three dosing models for the prediction of steady-state trough clozapine concentrations. Clin Biochem 2008; 41: 603-606

[923] Pearce GA, Day RO. Compliance with criteria necessary for effective drug concentration monitoring. Ther Drug Monit 1990; 12: 250-257

[924] Pedersen OL, Gram LF, Kristensen CB et al. Overdosage of antidepressants: clinical and pharmacokinetic aspects. Eur J Clin Pharmacol 1982; 23: 513-521

[925] Pellizzoni C, Poggesi I, Jorgensen NP et al. Pharmacokinetics of reboxetine in healthy volunteers. Single against repeated oral doses and lack of enzymatic alterations. Biopharm Drug Dispos 1996; 17: 623-633

[926] Perez ], Chiron C, Musial C et al. Stiripentol: Efficacy and tolerability in children with epilepsy. Epilepsia 1999; 40: 1618-1626

[927] Perlis RH, Fijal B, Dharia S et al. Failure to replicate genetic associations with antidepressant treatment response in duloxetine-treated patients. Biol Psychiatry 2010; 67: 1110-1113

[928] Perry PJ. Therapeutic drug monitoring of antipsychotics. Psychopharmacol Bull 2001; 35: 19-29

[929] Perry PJ, Browne JL, Alexander B et al. Relationship of free nortriptyline levels to therapeutic response. Acta Psychiatr Scand 1985; 72: $120-125$

[930] Perry PJ, Miller DD, Arndt SV et al. Clozapine and norclozapine plasma concentrations and clinical response of treatment-refractory schizophrenic patients. Am J Psychiatry 1991; 148: 231-235

[931] Perry PJ, Miller DD, Arndt SV et al. Haloperidol dosing requirements: the contribution of smoking and nonlinear pharmacokinetics. J Clin Psychopharmacol 1993; 13: 46-51

[932] Perry PJ, Pfohl BM, Holstad SG. The relationship between antidepressant response and tricyclic antidepressant plasma concentrations. A retrospective analysis of the literature using logistic regression analysis. Clin Pharmacokinet 1987; 13: 381-392

[933] Perry PJ, Sanger T, Beasley C. Olanzapine plasma concentrations and clinical response in acutely ill schizophrenic patients. J Clin Psychopharmacol 1997; 17: 472-477

[934] Perry PJ, Zeilmann C, Arndt S. Tricyclic antidepressant concentrations in plasma: An estimate of their sensitivity and specificity as a predictor of response. J Clin Psychopharmacol 1994; 14: 230-240

[935] Perucca E. Clinically relevant drug interactions with antiepileptic drugs. Br J Clin Pharmacol 2006; 61: 246-255

[936] Perucca E, Cloyd J, Critchley D et al. Rufinamide: Clinical pharmacokinetics and concentration-response relationships in patients with epilepsy. Epilepsia 2008; 49: 1123-1141

[937] Petit P, Lonjon R, Cociglio M et al. Carbamazepine and its 10,11-epoxide metabolite in acute mania: Clinical and pharmacokinetic correlates. Eur J Clin Pharmacol 1991; 41: 541-546
[938] Peyronneau MA, Delaforge M, Riviere R et al. High affinity of ergopeptides for cytochromes P450 3A. Importance of their peptide moiety for P450 recognition and hydroxylation of bromocriptine. Eur J Biochem 1994; 223: 947-956

[939] Pichini S, Papaseit E, Joya X et al. Pharmacokinetics and therapeutic drug monitoring of psychotropic drugs in pediatrics. Ther Drug Monit 2009; 31: 283-318

[940] Pierce DM, Franklin RA, Harry TV et al. Pharmacodynamic correlates of modified absorption: Studies with lormetazepam. Br J Clin Pharmacol 1984; 18: 31-35

[941] Pilla Reddy V, Kozielska M, Johnson M et al. Population pharmacokinetic-pharmacodynamic modeling of haloperidol in patients with schizophrenia using positive and negative syndrome rating scale. J Clin Psychopharmacol 2013; 33: 731-739

[942] Pilleri M, Antonini A. Novel levodopa formulations in the treatment of Parkinson's disease. Expert Rev Neurother 2014; 14: 143-149

[943] Piotrovsky V, Van Peer A, Van Osselaer N et al. Galantamine population pharmacokinetics in patients with Alzheimer's disease: modeling and simulations. J Clin Pharmacol 2003; 43: 514-523

[944] Plesnicar BK, Zalar B, Breskvar K et al. The influence of the CYP2D6 polymorphism on psychopathological and extrapyramidal symptoms in the patients on long-term antipsychotic treatment. J Psychopharmacol 2006; 20: 829-833

[945] Poggesi I, Benedetti MS, Whomsley R et al. Pharmacokinetics in special populations. Drug Metab Rev 2009; 41: 422-454

[946] Poppe C, Müller ST, Greil W et al. Pharmacotherapy for obsessive compulsive disorder in clinical practice - Data of 842 inpatients from the International AMSP Project between 1994 and 2012. J Affect Disord 2016; 200: 89-96

[947] Potgieter GE, Groenewoud G, Jordaan P] et al. Pharmacokinetics of pipamperone from three different tablet formulations. Arzneimittelforschung 2002; 52: 430-434

[948] Pounder D], Jones GR. Post-mortem drug redistribution-a toxicological nightmare. Forensic Sci Int 1990; 45: 253-263

[949] Pozzi M, Cattaneo D, Baldelli S et al. Therapeutic drug monitoring of second-generation antipsychotics in pediatric patients: An observational study in real-life settings. Eur ] Clin Pharmacol 2016; 72: 285-293

[950] Prakash C, Kamel A, Cui D et al. Identification of the major human liver cytochrome $\mathrm{P} 450$ isoform(s) responsible for the formation of the primary metabolites of ziprasidone and prediction of possible drug interactions. Br J Clin Pharmacol 2000; 49 (Suppl 1): 35S-42S

[951] Preskorn S, Ereshefsky L, Chiu YY et al. Effect of food on the pharmacokinetics of lurasidone: Results of two randomized, open-label, crossover studies. Hum Psychopharmacol 2013; 28 : 495-505

[952] Preskorn S, Patroneva A, Silman $\mathrm{H}$ et al. Comparison of the pharmacokinetics of venlafaxine extended release and desvenlafaxine in extensive and poor cytochrome P450 2D6 metabolizers. J Clin Psychopharmacol 2009; 29: 39-43

[953] Preskorn SH. CNS drug development. Part I: The early period of CNS drugs. J Psychiatr Pract 2010; 16: 334-339

[954] Preskorn SH. CNS drug development: Part II: Advances from the 1960s to the 1990s. J Psychiatr Pract 2010; 16: 413-415

[955] Preskorn SH. Patients who do not respond to the "usual" dose: Why Terry fell off the dose-response curve. J Psychiatr Pract 2009; 15: 460-466

[956] Preskorn SH. Pharmacokinetics and therapeutics of acute intramuscular ziprasidone. Clin Pharmacokinet 2005; 44: 1117-1133

[957] Preskorn SH. Practical application of therapeutic drug monitoring: A tale of two patients. J Psychiatr Pract 2008; 14: 301-306 
[958] Preskorn SH. Therapeutic Drug Monitoring (TDM) in psychiatry (part I): Why studies attempting to correlate drug concentration and antidepressant response don't work. J Psychiatr Pract 2014; 20: 133-137

[959] Preskorn SH. Tricyclic antidepressant plasma level monitoring: An improvement over the dose-response approach. J Clin Psychiatry 1986; 47: 24-30

[960] Preskorn SH, Burke MJ, Fast GA. Therapeutic drug monitoring. Principles and practice. Psychiatr Clin North Am 1993; 16: 611-645

[961] Preskorn SH, Fast GA. Therapeutic drug monitoring for antidepressants: Efficacy, safety, and cost effectiveness. J Clin Psychiatry 1991; 52 Suppl: 23-33

[962] Preskorn SH, Fast GA. Tricyclic antidepressant-induced seizures and plasma drug concentration. J Clin Psychiatry 1992; 53: 160-162

[963] Preskorn SH, Fleck RJ, Schroeder DH. Therapeutic drug monitoring of bupropion. Am J Psychiatry 1990; 147: 1690-1691

[964] Preskorn SH, Jerkovich GS. Central nervous system toxicity of tricyclic antidepressants: Phenomenology, course, risk factors, and role of therapeutic drug monitoring. J Clin Psychopharmacol 1990; 10: 88-95

[965] Proft F, Kopf J, Olmes D et al. SLC6A2 and SLC6A4 variants interact with venlafaxine serum concentrations to influence therapy outcome. Pharmacopsychiatry 2014; 47: 245-250

[966] Puech A, Fleurot O, Rein W. Amisulpride. and atypical antipsychotic, in the treatment of acute episodes of schizophrenia: A dose-ranging study vs. haloperidol. The Amisulpride Study Group. Acta Psychiatr Scand 1998; 98: 65-72

[967] Pumariega A], Nelson R, Rotenberg L. Varenicline-induced mixed mood and psychotic episode in a patient with a past history of depression. CNS Spectr 2008; 13: 511-514

[968] Puozzo C, Albin H, Vincon G et al. Pharmacokinetics of milnacipran in liver impairment. Eur J Drug Metab Pharmacokinet 1998; 23: 273-279

[969] Quinney SK, Haehner BD, Rhoades MB et al. Interaction between midazolam and clarithromycin in the elderly. $\mathrm{Br}$ ] Clin Pharmacol 2008; 65: 98-109

[970] Raaflaub J. On the pharmacokinetics of chlorprothixene in man. Experientia 1975; 31: 557-558

[971] Rabey JM, Oberman Z, Scharf M et al. Bromocriptine blood levels after the concomitant administration of levodopa, amantadine and biperiden in Parkinson's disease. Acta Neurol Scand 1990; 81: 411-415

[972] Radtke RA. Pharmacokinetics of levetiracetam. Epilepsia 2001; 42: (Suppl 4): 24-27

[973] Rao VA, Bishop M, Coppen A. Clinical state, plasma levels of haloperidol and prolactin: A correlation study in chronic schizophrenia. Br J Psychiatry 1980; 137: 518-521

[974] Rasmussen BB, Brosen K. Is therapeutic drug monitoring a case for optimizing clinical outcome and avoiding interactions of the selective serotonin reuptake inhibitors? Ther Drug Monit 2000; 22: 143-154

[975] Rauschenbach R, Gieschen H, Husemann M et al. Stable expression of human cytochrome P450 3A4 in V79 cells and its application for metabolic profiling of ergot derivatives. Eur J Pharmacol 1995; 293: 183-190

[976] Ravenstijn P, Remmerie B, Savitz A et al. Pharmacokinetics, safety, and tolerability of paliperidone palmitate 3-month formulation in patients with schizophrenia: A phase 1, single-dose, randomized, open-label study. J Clin Pharmacol 2016; 56: 330-339

[977] Ravva P, Gastonguay MR, Tensfeldt TG et al. Population pharmacokinetic analysis of varenicline in adult smokers. $\mathrm{Br}$ J Clin Pharmacol 2009; 68: 669-681
[978] Ray A, Tennakoon L, Keller J et al. ABCB1 (MDR1) predicts remission on P-gp substrates in chronic depression. Pharmacogenomics J 2015; 15: 332-339

[979] Rees JA. Clinical interpretation of pharmacokinetic data on dothiepin hydrochloride (Dosulepin, Prothiaden). J Int Med Res 1981; 9: 98-102

[980] Regenthal R, Krueger M, Koeppel C et al. Drug levels: therapeutic and toxic serum/plasma concentrations of common drugs. J Clin Monit Comput 1999; 15: 529-544

[981] Reidenberg P, Glue P, Banfield CR et al. Effects of felbamate on the pharmacokinetics of phenobarbital. Clin Pharmacol Ther 1995; 58 : 279-287

[982] Reimold M, Solbach C, Noda S et al. Occupancy of dopamine D(1), D (2) and serotonin $(2 \mathrm{~A})$ receptors in schizophrenic patients treated with flupentixol in comparison with risperidone and haloperidol. Psychopharmacology (Berl) 2007; 190: 241-249

[983] Reis M, Aamo T, Ahlner J et al. Reference concentrations of antidepressants. A compilation of postmortem and therapeutic levels. J Anal Toxicol 2007; 31: 254-264

[984] Reis M, Aamo T, Spigset O et al. Serum concentrations of antidepressant drugs in a naturalistic setting: Compilation based on a large therapeutic drug monitoring database. Ther Drug Monit 2009; 31 : 42-56

[985] Reis M, Aberg-Wistedt A, Agren $\mathrm{H}$ et al. Compliance with SSRI medication during 6 months of treatment for major depression: an evaluation by determination of repeated serum drug concentrations. J Affect Disord 2004; 82: 443-446

[986] Reis M, Akerblad AC, Ekselius L et al. Partial compliance as determined from plasma levels of sertraline and its metabolite in depressed patients in primary care. J Clin Psychopharmacol 2010; 30: 746-748

[987] Reis M, Cherma MD, Carlsson B et al. Therapeutic drug monitoring of escitalopram in an outpatient setting. Ther Drug Monit 2007; 29: 758-766

[988] Reis M, Lundmark J, Bengtsson F. Therapeutic drug monitoring of racemic citalopram: A 5-year experience in Sweden, 1992-1997. Ther Drug Monit 2003; 25: 183-191

[989] Reis M, Lundmark J, Bjork $\mathrm{H}$ et al. Therapeutic drug monitoring of racemic venlafaxine and its main metabolites in an everyday clinical setting. Ther Drug Monit 2002; 24: 545-553

[990] Reis M, Olsson G, Carlsson B et al. Serum levels of citalopram and its main metabolites in adolescent patients treated in a naturalistic clinical setting. J Clin Psychopharmacol 2002; 22: 406-413

[991] Reis M, Prochazka J, Sitsen A et al. Inter- and intraindividual pharmacokinetic variations of mirtazapine and its $\mathrm{N}$-demethyl metabolite in patients treated for major depressive disorder: A 6-month therapeutic drug monitoring study. Ther Drug Monit 2005; $27: 469-477$

[992] Remington G, Mamo D, Labelle A et al. A PET study evaluating dopamine $\mathrm{D} 2$ receptor occupancy for long-acting injectable risperidone. Am J Psychiatry 2006; 163: 396-401

[993] Renwick AB, Mistry H, Ball SE et al. Metabolism of Zaleplon by human hepatic microsomal cytochrome P450 isoforms. Xenobiotica 1998; 28: 337-348

[994] Riant P, Urien S, Albengres E et al. Effects of the binding of imipramine to erythrocytes and plasma proteins on its transport through the rat blood-brain barrier. J Neurochem 1988; 51 : 421-425

[995] Richens A, Banfield CR, Salfi M et al. Single and multiple dose pharmacokinetics of felbamate in the elderly. Br J Clin Pharmacol 1997; 44: 129-134 
[996] Richter T, Murdter TE, Heinkele G et al. Potent mechanism-based inhibition of human CYP2B6 by clopidogrel and ticlopidine. J Pharmacol Exp Ther 2004; 308: 189-197

[997] Riedel M, Schwarz M], Strassnig M et al. Risperidone plasma levels, clinical response and side-effects. Eur Arch Psychiatry Clin Neurosci 2005; 255: 261-268

[998] Rivas N, Buelga DS, Elger CE et al. Population pharmacokinetics of lamotrigine with data from therapeutic drug monitoring in German and Spanish patients with epilepsy. Ther Drug Monit 2008; 30: 483-489

[999] Rivera-Calimlim L, Castaneda L, Lasagna L. Effects of mode of management on plasma chlorpromazine in psychiatric patients. Clin Pharmacol Ther 1973; 14: 978-986

[1000] Roberts JK, Cook SF, Stockmann C et al. A Population pharmacokinetic analysis of dextroamphetamine in the plasma and hair of healthy adults. Clin Drug Investig 2015; 35: 633-643

[1001] Roberts RL, Joyce PR, Mulder RT et al. A common P-glycoprotein polymorphism is associated with nortriptyline-induced postural hypotension in patients treated for major depression. Pharmacogenomics J 2002; 2: 191-196

[1002] Robertson P, DeCory HH, Madan A et al. In vitro inhibition and induction of human hepatic cytochrome P450 enzymes by modafinil. Drug Metab Dispos 2000; 28: 664-671

[1003] Robertson P Jr., Hellriegel ET. Clinical pharmacokinetic profile of modafinil. Clin Pharmacokinet 2003; 42: 123-137

[1004] Robertson SM, Maldarelli F, Natarajan V et al. Efavirenz induces CYP2B6-mediated hydroxylation of bupropion in healthy subjects. Acquir Immune Defic Syndr 2008; 49: 513-519

[1005] Rocha JF, Falcao A, Santos A et al. Effect of opicapone and entacapone upon levodopa pharmacokinetics during three daily levodopa administrations. Eur J Clin Pharmacol 2014; 70: 10591071

[1006] Rocha JF, Santos A, Falcao A et al. Effect of moderate liver impairment on the pharmacokinetics of opicapone. Eur J Clin Pharmacol 2014; 70: 279-286

[1007] Rochat B, Kosel M, Boss G et al. Stereoselective biotransformation of the selective serotonin reuptake inhibitor citalopram and its demethylated metabolites by monoamine oxidases in human liver. Biochem Pharmacol 1998; 56: 15-23

[1008] Roelofsen EE, Wilhelm AJ, Sinjewel A et al. Toxicokinetics of dothiepin: 2 case reports. Ther Drug Monit 2008; 30: 638-641

[1009] Roesch B, Corcoran M, Haffey M et al. Pharmacokinetics of coadministration of guanfacine extended release and methylphenidate extended release. Drugs R D 2013; 13: 53-61

[1010] Roesch B, Corcoran ME, Fetterolf J et al. Pharmacokinetics of coadministered guanfacine extended release and lisdexamfetamine dimesylate. Drugs R D 2013; 13: 119-128

[1011] Rogers HL, Bhattaram A, Zineh I et al. CYP2D6 genotype information to guide pimozide treatment in adult and pediatric patients: Basis for the U.S. Food and Drug Administration's new dosing recommendations. J Clin Psychiatry 2012; 73: 1187-1190

[1012] Rogers SL, Doody RS, Mohs RC et al. Donepezil improves cognition and global function in Alzheimer disease: A 15-week, double-blind, placebo-controlled study. Donepezil Study Group. Arch Intern Med 1998; 158: 1021-1031

[1013] Rogers SL, Friedhoff LT. The efficacy and safety of donepezil in patients with Alzheimer's disease: Results of a US Multicentre, Randomized, Double-Blind, Placebo-Controlled Trial. The Donepezil Study Group. Dementia 1996; 7: 293-303

[1014] Rolan P, Sargentini-Maier ML, Pigeolet E et al. The pharmacokinetics, CNS pharmacodynamics and adverse event profile of brivaracetam after multiple increasing oral doses in healthy men. $\mathrm{Br}$ J Clin Pharmacol 2008; 66: 71-75
[1015] Roman M, Kronstrand R, Lindstedt D et al. Quantitation of seven low-dosage antipsychotic drugs in human postmortem blood using LC-MS-MS. J Anal Toxicol 2008; 32: 147-155

[1016] Ronfeld RA, Tremaine LM, Wilner KD. Pharmacokinetics of sertraline and its $\mathrm{N}$-demethyl metabolite in elderly and young male and female volunteers. Clin Pharmacokinet 1997; 32: (Suppl 1): 22-30

[1017] Rook EJ, Huitema AD, van den Brink W et al. Population pharmacokinetics of heroin and its major metabolites. Clin Pharmacokinet 2006; 45: 401-417

[1018] Rosenzweig P, Canal M, Patat A et al. A review of the pharmacokinetics, tolerability and pharmacodynamics of amisulpride in healthy volunteers. Hum Psychopharmacol 2002; 17: 1-13

[1019] Rotzinger S, Fang J, Baker GB. Trazodone is metabolized to m-chlorophenylpiperazine by CYP3A4 from human sources. Drug Metab Dispos 1998; 26: 572-575

[1020] Rougemont M, Ulrich S, Hiemke C et al. French summaries of product characteristics: Content in relation to therapeutic monitoring of psychotropic drugs. Fundam Clin Pharmacol 2010; 24: $377-384$

[1021] Ruan C], Li AN, Dong F et al. Single- and multiple-dose milnacipran pharmacokinetics in healthy han chinese volunteers. Clin Pharmacokinet 2016; 55: 889-896

[1022] Rudolph JL, Salow M], Angelini MC et al. The anticholinergic risk scale and anticholinergic adverse effects in older persons. Arch Intern Med 2008; 168: 508-513

[1023] Rudorfer MV, Potter WZ. The Role of Metabolites of Antidepressants in the Treatment of Depression. CNS Drugs 1997; 7: 273-312

[1024] Ruike Z, Junhua C, Wenxing P. In vitro and in vivo evaluation of the effects of duloxetine on P-gp function. Hum Psychopharmacol 2010; 25: 553-559

[1025] Ruottinen HM, Rinne UK. Effect of one month's treatment with peripherally acting catechol-O-methyltransferase inhibitor, entacapone, on pharmacokinetics and motor response to levodopa in advanced parkinsonian patients. Clin Neuropharmacol 1996; 19: 222-233

[1026] Sachse J, Härtter S, Hiemke C. Automated determination of ziprasidone by HPLC with column switching and spectrophotometric detection. Ther Drug Monit 2005; 27: 158-162

[1027] Sachse J, Härtter S, Weigmann $\mathrm{H}$ et al. Automated determination of amisulpride by liquid chromatography with column switching and spectrophotometric detection. J Chromatogr B Analyt Technol Biomed Life Sci 2003; 784: 405-410

[1028] Sachse J, Koller J, Härtter $S$ et al. Automated analysis of quetiapine and other antipsychotic drugs in human blood by high performance-liquid chromatography with column-switching and spectrophotometric detection. J Chromatogr B Analyt Technol Biomed Life Sci 2006; 830: 342-348

[1029] Sagar KA, Smyth MR. Bioavailability studies of oral dosage forms containing levodopa and carbidopa using column-switching chromatography followed by electrochemical detection. Analyst 2000; 125: 439-445

[1030] Sage JI, Mark MH. Pharmacokinetics of continuous-release carbidopa/levodopa. Clin Neuropharmacol 1994; 17: (Suppl 2): S1-S6

[1031] Sainati SM, Hubbard JW, Chi E et al. Safety, tolerability, and effect of food on the pharmacokinetics of iloperidone (HP 873), a potential atypical antipsychotic. J Clin Pharmacol 1995; 35: 713-720

[1032] Saint-Marcoux F, Sauvage FL, Marquet P. Current role of LC-MS in therapeutic drug monitoring. Anal Bioanal Chem 2007; 388: $1327-1349$

[1033] Saivin S, Hulot T, Chabac S et al. Clinical pharmacokinetics of acamprosate. Clin Pharmacokinet 1998; 35: 331-345 
[1034] Sajatovic M, Velligan DI, Weiden PJ et al. Measurement of psychiatric treatment adherence. J Psychosom Res 2010; 69: 591-599

[1035] Sakka SG, Kuethe F, Demme U et al. Intoxication with a tricyclic antidepressant. Anaesthesist 2007; 56: 581-586

[1036] Sakolsky DJ, Perel JM, Emslie G] et al. Antidepressant exposure as a predictor of clinical outcomes in the Treatment of Resistant Depression in Adolescents (TORDIA) study. J Clin Psychopharmacol 2011; 31: 92-97

[1037] Salazar DE, Frackiewicz E], Dockens R et al. Pharmacokinetics and tolerability of buspirone during oral administration to children and adolescents with anxiety disorder and normal healthy adults. J Clin Pharmacol 2001; 41: 1351-1358

[1038] Sallee FR, Pollock BG. Clinical pharmacokinetics of imipramine and desipramine. Clin Pharmacokinet 1990; 18: 346-364

[1039] Sallee FR, Pollock BG, Stiller RL et al. Pharmacokinetics of pimozide in adults and children with Tourette's syndrome. J Clin Pharmacol 1987; $27:$ 776-781

[1040] Salva P, Costa J. Clinical pharmacokinetics and pharmacodynamics of zolpidem. Therapeutic implications. Clin Pharmacokinet 1995; 29: $142-153$

[1041] Samer CF, Lorenzini KI, Rollason V et al. Applications of CYP450 testing in the clinical setting. Mol Diagn Ther 2013; 17: 165-184

[1042] Sargentini-Maier ML, Espie P, Coquette A et al. Pharmacokinetics and metabolism of 14C-brivaracetam, a novel SV2A ligand, in healthy subjects. Drug Metab Dispos 2008; 36: 36-45

[1043] Sarginson JE, Lazzeroni LC, Ryan HS et al. ABCB1 (MDR1) polymorphisms and antidepressant response in geriatric depression. Pharmacogenet Genomics 2010; 20: 467-475

[1044] Saruwatari J, Ogusu N, Shimomasuda M et al. Effects of CYP2C19 and $\mathrm{P} 450$ oxidoreductase polymorphisms on the population pharmacokinetics of clobazam and $\mathrm{N}$-desmethylclobazam in japanese patients with epilepsy. Ther Drug Monit 2014; 36 : 302-309

[1045] Sattler A, Schaefer M, May TW et al. Fluctuation of lacosamide serum concentrations during the day and occurrence of adverse drug reactions--first clinical experience. Epilepsy Res 2011; 95 : 207-212

[1046] Sauer JM, Ring B], Witcher JW. Clinical pharmacokinetics of atomoxetine. Clin Pharmacokinet 2005; 44: 571-590

[1047] Sauvage FL, Gaulier JM, Lachatre $G$ et al. Pitfalls and prevention strategies for liquid chromatography-tandem mass spectrometry in the selected reaction-monitoring mode for drug analysis. Clin Chem 2008; 54: 1519-1527

[1048] Schafer A, Hiemke C, Baumann P. Consensus guideline for therapeutic drug monitoring in psychiatry (2004): Bibliometric analysis of citations for the period 2004-2011. Nord J Psychiatry 2016; 70: 202-207

[1049] Schall U, Pries E, Katta T et al. Pharmacokinetic and pharmacodynamic interactions in an outpatient maintenance therapy of intravenous heroin users with levomethadone. Addict Biol 1996; 1: 105-113

[1050] Scharfetter J, Fischer P. QTc prolongation induced by intravenous sedation with Haloperidol, Prothipendyl and Lorazepam. Neuropsychiatr 2014; 28: 1-5

[1051] Schatzberg AF, DeBattista C, Lazzeroni LC et al. ABCB1 Genetic effects on antidepressant outcomes: A report from the iSPOT-D trial. Am J Psychiatry 2015; 172: 751-759

[1052] Schifano F. Misuse and abuse of pregabalin and gabapentin: cause for concern? CNS Drugs 2014; 28: 491-496

[1053] Schoemaker R, Wade JR, Stockis A. Brivaracetam population pharmacokinetics and exposure-response modeling in adult subjects with partial-onset seizures. J Clin Pharmacol 2016; 56: 1591-1602

[1054] Schoretsanitis G, Haen E, Gründer G et al. Pharmacokinetic drug-drug interactions of mood stabilizers and risperidone in patients under combined treatment. J Clin Psychopharmacol 2016; 36: $554-561$

[1055] Schoretsanitis G, Haen E, Stegmann B et al. Effect of smoking on risperidone pharmacokinetics - A multifactorial approach to better predict the influence on drug metabolism. Schizophr Res 2017; 185: 51-57

[1056] Schoretsanitis G, Stegmann B, Hiemke C et al. Pharmacokinetic patterns of risperidone-associated adverse drug reactions. Eur J Clin Pharmacol 2016; 72: 1091-1098

[1057] Schubert I, Kupper-Nybelen J, Ihle P et al. Prescribing potentially inappropriate medication (PIM) in Germany's elderly as indicated by the PRISCUS list. An analysis based on regional claims data. Pharmacoepidemiol Drug Saf 2013; 22: 719-727

[1058] Schulz M, Iwersen-Bergmann S, Andresen $\mathrm{H}$ et al. Therapeutic and toxic blood concentrations of nearly 1,000 drugs and other xenobiotics. Crit Care 2012; 16: R136

[1059] Schulze TG, Alda M, Adli M et al. The International Consortium on Lithium Genetics (ConLiGen): An initiative by the NIMH and IGSLI to study the genetic basis of response to lithium treatment. Neuropsychobiology 2010; 62: 72-78

[1060] Schwartz JB. The current state of knowledge on age, sex, and their interactions on clinical pharmacology. Clin Pharmacol Ther 2007; 82: 87-96

[1061] Schwartz MA, Postma E. Metabolism of flurazepam, a benzodiazepine, in man and dog. J Pharm Sci 1970; 59: 1800-1806

[1062] Schwarz V, Reis O, Glaser T et al. Therapeutic drug monitoring of zuclopenthixol in a double-blind placebo-controlled discontinuation study in adults with intellectual disabilities and aggressive behaviour. Pharmacopsychiatry 2014; 47: 29-32

[1063] Schwarzenbach F, Netillard C, Demoly P et al. Antidepressant response and fluvoxamine plasma concentrations: A pilot study. Pharm World Sci 2003; 25: 27-29

[1064] Scordo MG, Spina E, Dahl ML et al. Influence of CYP2C9, 2C19 and 2D6 genetic polymorphisms on the steady-state plasma concentrations of the enantiomers of fluoxetine and norfluoxetine. Basic Clin Pharmacol Toxicol 2005; 97: 296-301

[1065] The Scottish First Episode Schizophrenia Study II. Treatment: Pimozide versus flupenthixol. The scottish schizophrenia research group. Br J Psychiatry 1987; 150: 334-338

[1066] Segman RH, Heresco-Levy U, Finkel B et al. Association between the serotonin $2 \mathrm{~A}$ receptor gene and tardive dyskinesia in chronic schizophrenia. Mol Psychiatry 2001; 6: 225-229

[1067] Segman RH, Heresco-Levy U, Finkel B et al. Association between the serotonin $2 \mathrm{C}$ receptor gene and tardive dyskinesia in chronic schizophrenia: additive contribution of 5-HT2Cser and DRD3gly alleles to susceptibility. Psychopharmacology (Berl) 2000; 152: 408-413

[1068] Seiler W, Wetzel H, Hillert A et al. Pharmacokinetics and bioavailability of benperidol in schizophrenic patients after intravenous and two different kinds of oral application. Psychopharmacology (Berl) 1994; 116: 457-463

[1069] Self TH, Chrisman CR, Baciewicz AM et al. Isoniazid drug and food interactions. Am J Med Sci 1999; 317: 304-311

[1070] Seree EJ, Pisano PJ, Placidi M et al. Identification of the human and animal hepatic cytochromes P450 involved in clonazepam metabolism. Fundam Clin Pharmacol 1993; 7: 69-75

[1071] Serretti A, Kato M, De Ronchi D et al. Meta-analysis of serotonin transporter gene promoter polymorphism (5-HTTLPR) association 
with selective serotonin reuptake inhibitor efficacy in depressed patients. Mol Psychiatry 2007; 12: 247-257

[1072] Service JA, Waring WS. QT Prolongation and delayed atrioventricular conduction caused by acute ingestion of trazodone. Clin Toxicol (Phila) 2008; 46: 71-73

[1073] Shams M, Hiemke C, Härtter S. Therapeutic drug monitoring of the antidepressant mirtazapine and its $\mathrm{N}$-demethylated metabolite in human serum. Ther Drug Monit 2004; 26: 78-84

[1074] Shams ME, Arneth B, Hiemke $C$ et al. CYP2D6 polymorphism and clinical effect of the antidepressant venlafaxine. J Clin Pharm Ther 2006; 31: 493-502

[1075] Shang DW, Li LJ, Wang XP et al. Population pharmacokinetic/ pharmacodynamic model of clozapine for characterizing the relationship between accumulated exposure and PANSS scores in patients with schizophrenia. Ther Drug Monit 2014; 36: 378-386

[1076] Sharma S, Joshi S, Chadda RK. Therapeutic drug monitoring of lithium in patients with bipolar affective disorder: Experiences from a tertiary care hospital in India. Am J Ther 2009; 16: 393-397

[1077] Sharma S, Joshi S, Mukherji S et al. Therapeutic drug monitoring: appropriateness and clinical utility in neuropsychiatry practice. Am J Ther 2009; 16: 11-16

[1078] Sheehan JJ, Reilly KR, Fu DJ et al. Comparison of the peak-to-trough fluctuation in plasma concentration of long-acting injectable antipsychotics and their oral equivalents. Innov Clin Neurosci 2012; 9: $17-23$

[1079] Shimoda K, Jerling M, Bottiger $Y$ et al. Pronounced differences in the dispositon of clomipramine between Japanese and Swedish patients.

J Clin Psychopharmacol 1999; 19: 393-400

[1080] Shin C, Han C, Pae CU et al. Precision medicine for psychopharmacology: a general introduction. Expert Rev Neurother 2016; 16: 831-839

[1081] Shin JG, Soukhova N, Flockhart DA. Effect of antipsychotic drugs on human liver cytochrome P-450 (CYP) isoforms in vitro: Preferential inhibition of CYP2D6. Drug Metab Dispos 1999; 27: 1078-1084

[1082] Shinderman M, Maxwell S, Brawand-Amey M et al. Cytochrome P4503A4 metabolic activity, methadone blood concentrations, and methadone doses. Drug Alcohol Depend 2003; 69: 205-211

[1083] Shiraga T, Kaneko H, Iwasaki K et al. Identification of cytochrome P450 enzymes involved in the metabolism of zotepine, an antipsychotic drug, in human liver microsomes. Xenobiotica 1999; 29: $217-229$

[1084] Shiran MR, Hassanzadeh-Khayyat M, Iqbal MZ et al. Can saliva replace plasma for the monitoring of methadone? Ther Drug Monit 2005; 27: 580-586

[1085] Shram M], Quinn AM, Chen N et al. Differences in the in vitro and in vivo pharmacokinetic profiles of once-daily modified-release methylphenidate formulations in Canada: Examination of current bioequivalence criteria. Clin Ther 2012; 34: 1170-1181

[1086] Shua-Haim J, Smith J, Picard F et al. Steady-state pharmacokinetics of rivastigmine in patients with mild to moderate Alzheimer's disease not affected by co-administration of memantine: An open-label, crossover, single-centre study. Clin Drug Investig 2008; 28: $361-374$

[1087] Sidhu J, Priskorn M, Poulsen M et al. Steady-state pharmacokinetics of the enantiomers of citalopram and its metabolites in humans. Chirality 1997; 9: 686-692

[1088] Sigurdsson HP, Hefner G, Ben-Omar N et al. Steady-state serum concentrations of venlafaxine in patients with late-life depression. Impact of age, sex and BMI. J Neural Transm (Vienna) 2015; 122: 721-729
[1089] Simmons SA, Perry P], Rickert ED et al. Cost-benefit analysis of prospective pharmacokinetic dosing of nortriptyline in depressed inpatients. J Affect Disord 1985; 8: 47-53

[1090] Simons FE, Watson WT, Chen XY et al. The pharmacokinetics and pharmacodynamics of hydroxyzine in patients with primary biliary cirrhosis. J Clin Pharmacol 1989; 29: 809-815

[1091] Simons KJ, Watson WT, Martin TJ et al. Diphenhydramine: pharmacokinetics and pharmacodynamics in elderly adults, young adults, and children. J Clin Pharmacol 1990; 30: 665-671

[1092] Sindrup SH, Brosen K, Gram LF. Pharmacokinetics of the selective serotonin reuptake inhibitor paroxetine: Nonlinearity and relation to the sparteine oxidation polymorphism. Clin Pharmacol Ther 1992; 51: 288-295

[1093] Siva N. Tackling the booming trade in counterfeit drugs. Lancet 2010; 376: 1725-1726

[1094] Sjoqvist F. Development of clinical pharmacology as a medical speciality in Europe - the roles of WHO, IUPHAR and EACPT. Basic Clin Pharmacol Toxicol 2014; 115: 172-178

[1095] Skinner MD, Lahmek P, Pham H et al. Disulfiram efficacy in the treatment of alcohol dependence: A meta-analysis. PLoS One 2014; 9: e87366

[1096] Skinner MH, Kuan HY, Pan A et al. Duloxetine is both an inhibitor and a substrate of cytochrome P4502D6 in healthy volunteers. Clin Pharmacol Ther 2003; 73: 170-177

[1097] Skinner MH, Kuan HY, Skerjanec A et al. Effect of age on the pharmacokinetics of duloxetine in women. $\mathrm{Br}$ J Clin Pharmacol 2004; 57: 54-61

[1098] Skogh E, Bengtsson F, Nordin C. Could discontinuing smoking be hazardous for patients administered clozapine medication? A case report. Ther Drug Monit 1999; 21: 580-582

[1099] Skogh E, Reis M, Dahl ML et al. Therapeutic drug monitoring data on olanzapine and its $\mathrm{N}$-demethyl metabolite in the naturalistic clinical setting. Ther Drug Monit 2002; 24: 518-526

[1100] Small JG, Hirsch SR, Arvanitis LA et al. Quetiapine in patients with schizophrenia. A high- and low-dose double-blind comparison with placebo. Seroquel Study Group. Arch Gen Psychiatry 1997; 54: 549-557

[1101] Smith JC, Curry SC. Prolonged toxicity after amitriptyline overdose in a patient deficient in CYP2D6 activity. J Med Toxicol 2011; 7: $220-223$

[1102] Smith RB, Divoll M, Gillespie WR et al. Effect of subject age and gender on the pharmacokinetics of oral triazolam and temazepam. J Clin Psychopharmacol 1983; 3: 172-176

[1103] Smith RB, Kroboth PD, Vanderlugt JT et al. Pharmacokinetics and pharmacodynamics of alprazolam after oral and IV administration. Psychopharmacology (Berl) 1984; 84: 452-456

[1104] Smith SW. Chiral toxicology: it's the same thing...only different. Toxicol Sci 2009; 110: 4-30

[1105] Snoeck E, Van Peer A, Sack M et al. Influence of age, renal and liver impairment on the pharmacokinetics of risperidone in man. Psychopharmacology (Berl) 1995; 122: 223-229

[1106] Sogaard B, Mengel H, Rao N et al. The pharmacokinetics of escitalopram after oral and intravenous administration of single and multiple doses to healthy subjects. J Clin Pharmacol 2005; 45: $1400-1406$

[1107] Soldin OP, Mattison DR. Sex differences in pharmacokinetics and pharmacodynamics. Clin Pharmacokinet 2009; 48: 143-157

[1108] Someya T, Muratake T, Hirokane G et al. Interindividual variation in bromperidol metabolism and relationship to therapeutic effects. J Clin Psychopharmacol 2000; 20: 175-180

[1109] Sondergaard Khinchi M, Nielsen KA, Dahl M et al. Lamotrigine therapeutic thresholds. Seizure 2008; 17: 391-395 
[1110] Song L, Du Q, Jiang X et al. Effect of CYP1A2 polymorphism on the pharmacokinetics of agomelatine in Chinese healthy male volunteers.

J Clin Pharm Ther 2014; 39: 204-209

[1111] Soni SD. Fluspirilene in the treatment of non-hospitalized schizophrenic patients. Curr Med Res Opin 1977; 4: 645-649

[1112] Sorgel F, Mahr G, Granneman GR et al. Effects of 2 quinolone antibacterials, temafloxacin and enoxacin, on theophylline pharmacokinetics. Clin Pharmacokinet 1992; 22: (Suppl 1): 65-74

[1113] Spanarello S, La Ferla T. The pharmacokinetics of long-acting antipsychotic medications. Curr Clin Pharmacol 2014; 9: 310-317

[1114] Sparshatt A, Taylor D, Patel MX et al. Amisulpride - dose, plasma concentration, occupancy and response: Implications for therapeutic drug monitoring. Acta Psychiatr Scand 2009; 120: 416-428

[1115] Sparshatt A, Taylor D, Patel MX et al. A systematic review of aripiprazole-dose, plasma concentration, receptor occupancy, and response: implications for therapeutic drug monitoring. J Clin Psychiatry 2010; 71: 1447-1456

[1116] Spencer TJ, Biederman J, Ciccone PE et al. PET study examining pharmacokinetics, detection and likeability, and dopamine transporter receptor occupancy of short- and long-acting oral methylphenidate. Am J Psychiatry 2006; 163: 387-395

[1117] Spencer T], Bonab AA, Dougherty DD et al. A PET study examining pharmacokinetics and dopamine transporter occupancy of two long-acting formulations of methylphenidate in adults. Int J Mol Med 2010; 25: 261-265

[1118] Spies M, Knudsen GM, Lanzenberger R et al. The serotonin transporter in psychiatric disorders: insights from PET imaging. Lancet Psychiatry 2015; 2: 743-755

[1119] Spigset O, Hagg S, Stegmayr B et al. Citalopram pharmacokinetics in patients with chronic renal failure and the effect of haemodialysis. Eur J Clin Pharmacol 2000; 56: 699-703

[1120] Spina E, Avenoso A, Facciola G et al. Relationship between plasma risperidone and 9-hydroxyrisperidone concentrations and clinical response in patients with schizophrenia. Psychopharmacology (Berl) 2001; 153: 238-243

[1121] Spina E, Birgersson C, von Bahr C et al. Phenotypic consistency in hydroxylation of desmethylimipramine and debrisoquine in healthy subjects and in human liver microsomes. Clin Pharmacol Ther 1984; 36: 677-682

[1122] Spina E, Pisani F, Perucca E. Clinically significant pharmacokinetic drug interactions with carbamazepine. An update. Clin Pharmacokinet 1996; 31: 198-214

[1123] Spinewine A, Schmader KE, Barber $\mathrm{N}$ et al. Appropriate prescribing in elderly people: How well can it be measured and optimised? Lancet 2007; 370: 173-184

[1124] Spivak AM, Andrade A, Eisele E et al. A pilot study assessing the safety and latency-reversing activity of disulfiram in HIV-1-infected adults on antiretroviral therapy. Clin Infect Dis 2014; 58: 883-890

[1125] Sproule BA, Hardy BG, Shulman KI. Differential pharmacokinetics of lithium in elderly patients. Drugs Aging 2000; 16: 165-177

[1126] Spyker DA, Riesenberg RA, Cassella JV. Multiple dose pharmacokinetics of inhaled loxapine in subjects on chronic, stable antipsychotic regimens. J Clin Pharmacol 2015; 55: 985-994

[1127] Sramek J], Cutler NR. The impact of gender on antidepressants. Curr Top Behav Neurosci 2011; 8: 231-249

[1128] Sridharan K, Sivaramakrishnan G. Interaction of Citrus Juices with Cyclosporine: Systematic Review and Meta-Analysis. Eur J Drug Metab Pharmacokinet 2016; 41: 665-673

[1129] Stamm TJ, Becker D, Sondergeld LM et al. Prediction of antidepressant response to venlafaxine by a combination of early response assessment and therapeutic drug monitoring. Pharmacopsychiatry 2014; 47: 174-179
[1130] Stassen H, Anghelescu IG, Angst ] et al. Predicting response to psychopharmacological treatment: Survey of recent results. Pharmacopsychiatry 2011; 44: 263-272

[1131] Stassen HH, Angst J, Hell D et al. Is there a common resilience mechanism underlying antidepressant drug response? Evidence from 2848 patients. J Clin Psychiatry 2007; 68: 1195-1205

[1132] Stead AH, Moffat AC. A collection of therapeutic, toxic and fatal blood drug concentrations in man. Hum Toxicol 1983; 2: 437-464

[1133] Stegmann B, Dorfelt A, Haen E. Quantification of Methylphenidate, Dexamphetamine, and Atomoxetine in Human Serum and Oral Fluid by HPLC With Fluorescence Detection. Ther Drug Monit 2016; 38 : 98-107

[1134] Steimer W. Besondere Bedeutung der Präanalytik und Interpretatoion bei der Bestimmung von Arzneimittelkonzentrationen. DBI 2004; 24: 147-157

[1135] Steimer W, Potter JM. Pharmacogenetic screening and therapeutic drugs. Clin Chim Acta 2002; 315: 137-155

[1136] Steimer W, Zopf K, von Amelunxen S et al. Amitriptyline or not, that is the question: pharmacogenetic testing of CYP2D6 and CYP2C19 identifies patients with low or high risk for side effects in amitriptyline therapy. Clin Chem 2005; 51: 376-385

[1137] Stenkrona P, Halldin C, Lundberg J. 5-HTT and 5-HT(1A) receptor occupancy of the novel substance vortioxetine (Lu AA21004). A PET study in control subjects. Eur Neuropsychopharmacol 2013; 23: 1190-1198

[1138] Stepanova D, Beran RG. The benefits of antiepileptic drug (AED) blood level monitoring to complement clinical management of people with epilepsy. Epilepsy Behav 2015; 42: 7-9

[1139] Stepanova D, Beran RG. Measurement of levetiracetam drug levels to assist with seizure control and monitoring of drug interactions with other anti-epileptic medications (AEMs). Seizure 2014; 23 : 371-376

[1140] Stephenson A, Anderson GM, Rochon P. Off-label prescribing in older people: The need for increased awareness and caution. Drugs Aging 2012; 29: 435-436

[1141] Stieffenhofer V, Hiemke C. Pharmacogenetics, therapeutic drug monitoring and non compliance. Ther Umsch 2010; 67: 309-315

[1142] Stieffenhofer V, Saglam H, Schmidtmann I et al. Clozapine plasma level monitoring for prediction of rehospitalization schizophrenic outpatients. Pharmacopsychiatry 2011; 44: 55-59

[1143] Stingl J, Viviani R. Polymorphism in CYP2D6 and CYP2C19, members of the cytochrome P450 mixed-function oxidase system, in the metabolism of psychotropic drugs. J Intern Med 2015; 277: 167-177

[1144] Stingl JC, Bartels H, Viviani R et al. Relevance of UDP-glucuronosyltransferase polymorphisms for drug dosing: A quantitative systematic review. Pharmacol Ther 2014; 141: 92-116

[1145] Stingl JC, Brockmoller J, Viviani R. Genetic variability of drug-metabolizing enzymes: The dual impact on psychiatric therapy and regulation of brain function. Mol Psychiatry 2013; 18: 273-287

[1146] Stock B, Spiteller G. Metabolism of antiparkinson drugs. An example of competitive hydroxylation. Arzneimittelforschung 1979; 29 : 610-615

[1147] Stockis A, Watanabe S, Rouits E et al. Brivaracetam single and multiple rising oral dose study in healthy Japanese participants: Influence of CYP2C19 genotype. Drug Metab Pharmacokinet 2014; 29: 394-399

[1148] Stollberger C, Huber JO, Finsterer J. Antipsychotic drugs and QT prolongation. Int Clin Psychopharmacol 2005; 20: 243-251

[1149] Stormer E, Brockmoller J, Roots I et al. Cytochrome P-450 enzymes and $\mathrm{FMO} 3$ contribute to the disposition of the antipsychotic drug perazine in vitro. Psychopharmacology (Berl) 2000; 151: 312-320 
[1150] Stormer E, von Moltke LL, Shader RI et al. Metabolism of the antidepressant mirtazapine in vitro: Contribution of cytochromes P-450 1A2, 2D6, and 3A4. Drug Metab Dispos 2000; 28: 1168-1175

[1151] Stout SM, Cimino NM. Exogenous cannabinoids as substrates, inhibitors, and inducers of human drug metabolizing enzymes: A systematic review. Drug Metab Rev 2014; 46: 86-95

[1152] Suhara T, Takano A, Sudo Y et al. High levels of serotonin transporter occupancy with low-dose clomipramine in comparative occupancy study with fluvoxamine using positron emission tomography. Arch Gen Psychiatry 2003; 60: 386-391

[1153] Sutfin TA, Perini GI, Molnar G et al. Multiple-dose pharmacokinetics of imipramine and its major active and conjugated metabolites in depressed patients. J Clin Psychopharmacol 1988; 8: 48-53

[1154] Sutton D, Butler AM, Nadin L et al. Role of CYP3A4 in human hepatic diltiazem N-demethylation: Inhibition of CYP3A4 activity by oxidized diltiazem metabolites. J Pharmacol Exp Ther 1997; 282: 294-300

[1155] Suzuki A, Otani K, Ishida M et al. No interaction between desipramine and bromperidol. Prog Neuropsychopharmacol Biol Psychiatry 1996; 20: 1265-1271

[1156] Suzuki A, Otani K, Ishida M et al. Increased plasma concentrations of bromperidol and its reduced metabolite with levomepromazine, but not with thioridazine. Ther Drug Monit 1997; 19: 261-264

[1157] Suzuki T, Mihara K, Nakamura A et al. Effects of genetic polymorphisms of CYP2D6, CYP3A5, and ABCB1 on the steady-state plasma concentrations of aripiprazole and its active metabolite, dehydroaripiprazole, in Japanese patients with schizophrenia. Ther Drug Monit 2014; 36: 651-655

[1158] Suzuki Y, Fukui N, Sawamura K et al. Concentration-response relationship for fluvoxamine using remission as an endpoint: $\mathrm{A}$ receiver operating characteristics curve analysis in major depression. J Clin Psychopharmacol 2008; 28: 325-328

[1159] Svirbely JR, Speicher CE. The importance of request and report forms in the interpretation of therapeutic drug monitoring data. Ther Drug Monit 1980; 2: 211-216

[1160] Sweet RA, Pollock BG, Kirshner M et al. Pharmacokinetics of singleand multiple-dose bupropion in elderly patients with depression. J Clin Pharmacol 1995; 35: 876-884

[1161] Sweet RA, Pollock BG, Mulsant BH et al. Pharmacologic profile of perphenazine's metabolites. J Clin Psychopharmacol 2000; 20: 181-187

[1162] Szegedi A, Jansen WT, van Willigenburg AP et al. Early improvement in the first 2 weeks as a predictor of treatment outcome in patients with major depressive disorder: A meta-analysis including 6562 patients. J Clin Psychiatry 2009; 70: 344-353

[1163] Szymura-Oleksiak J, Wyska E, Wasieczko A. Pharmacokinetic interaction between imipramine and carbamazepine in patients with major depression. Psychopharmacology (Berl) 2001; 154: 38-42

[1164] Takahashi LH, Huie K, Spyker DA et al. Effect of smoking on the pharmacokinetics of inhaled loxapine. Ther Drug Monit 2014; 36: 618-623

[1165] Takano A, Halldin C, Farde L. SERT and NET occupancy by venlafaxine and milnacipran in nonhuman primates: A PET study. Psychopharmacology (Berl) 2013; 226: 147-153

[1166] Takano A, Suhara T, Ichimiya T et al. Time course of in vivo 5-HTT transporter occupancy by fluvoxamine. J Clin Psychopharmacol 2006; 26: 188-191

[1167] Takano A, Suzuki K, Kosaka J et al. A dose-finding study of duloxetine based on serotonin transporter occupancy. Psychopharmacology (Berl) 2006; 185: 395-399

[1168] Takekita Y, Fabbri C, Kato M et al. HTR1A Polymorphisms and Clinical Efficacy of Antipsychotic Drug Treatment in Schizophrenia: A Meta-Analysis. Int J Neuropsychopharmacol 2016; 19
[1169] Tan L, Yu JT, Sun YP et al. The influence of cytochrome oxidase CYP2A6, CYP2B6, and CYP2C9 polymorphisms on the plasma concentrations of valproic acid in epileptic patients. Clin Neurol Neurosurg 2010; 112: 320-323

[1170] Tanaka E, Kurata N, Yasuhara H. How useful is the "cocktail approach" for evaluating human hepatic drug metabolizing capacity using cytochrome $\mathrm{P} 450$ phenotyping probes in vivo? J Clin Pharm Ther 2003; 28: 157-165

[1171] Tanaka E, Misawa S. Pharmacokinetic interactions between acute alcohol ingestion and single doses of benzodiazepines, and tricyclic and tetracyclic antidepressants - an update. J Clin Pharm Ther 1998; 23: 331-336

[1172] Tanaka O, Kondo T, Otani K et al. Single oral dose kinetics of zotepine and its relationship to prolactin response and side effects. Ther Drug Monit 1998; 20: 117-119

[1173] Tapaninen T, Backman JT, Kurkinen KJ et al. Itraconazole, a P-glycoprotein and CYP3A4 inhibitor, markedly raises the plasma concentrations and enhances the renin-inhibiting effect of aliskiren. J Clin Pharmacol 2011; 51: 359-367

[1174] Taright N, Mentre F, Mallet A et al. Nonparametric estimation of population characteristics of the kinetics of lithium from observational and experimental data: Individualization of chronic dosing regimen using a new Bayesian approach. Ther Drug Monit 1994; 16: 258-269

[1175] Tasker TC, Kaye CM, Zussman BD et al. Paroxetine plasma levels: lack of correlation with efficacy or adverse events. Acta Psychiatr Scand Suppl 1989; 350: 152-155

[1176] Tateishi T, Watanabe M, Kumai T et al. CYP3A is responsible for $\mathrm{N}$-dealkylation of haloperidol and bromperidol and oxidation of their reduced forms by human liver microsomes. Life Sci 2000; 67: 2913-2920

[1177] Taurines R, Burger R, Wewetzer $C$ et al. The relation between dosage, serum concentrations, and clinical outcome in children and adolescents treated with sertraline: A naturalistic study. Ther Drug Monit 2013; 35: 84-91

[1178] Taylor D. Antidepressant drugs and cardiovascular pathology: A clinical overview of effectiveness and safety. Acta Psychiatr Scand 2008; 118: 434-442

[1179] Taylor D. Psychopharmacology and adverse effects of antipsychotic long-acting injections: A review. Br J Psychiatry Suppl 2009; 52: S13-S19

[1180] Taylor G, Houston JB, Shaffer ] et al. Pharmacokinetics of promethazine and its sulphoxide metabolite after intravenous and oral administration to man. Br J Clin Pharmacol 1983; 15: 287-293

[1181] Taylor M], Sen S, Bhagwagar Z. Antidepressant response and the serotonin transporter gene-linked polymorphic region. Biol Psychiatry 2010; 68: 536-543

[1182] Temesvari M, Kobori L, Paulik J et al. Estimation of drug-metabolizing capacity by cytochrome P450 genotyping and expression. J Pharmacol Exp Ther 2012; 341: 294-305

[1183] Tetrault JM, McCance-Katz EF, Moody DE et al. The impact of recent cocaine use on plasma levels of methadone and buprenorphine in patients with and without HIV-infection. J Subst Abuse Treat 2015; 51: 70-74

[1184] Thanacoody RH, Daly AK, Reilly JG et al. Factors affecting drug concentrations and QT interval during thioridazine therapy. Clin Pharmacol Ther 2007; 82: 555-565

[1185] Theisen FM, Haberhausen M, Schulz E et al. Serum levels of olanzapine and its $\mathrm{N}$-desmethyl and 2-hydroxymethyl metabolites in child and adolescent psychiatric disorders: Effects of dose, diagnosis, age, sex, smoking, and comedication. Ther Drug Monit 2006; 28: 750-759 
[1186] Theunissen EL, Street D, Hojer AM et al. A randomized trial on the acute and steady-state effects of a new antidepressant, vortioxetine (Lu AA21004), on actual driving and cognition. Clin Pharmacol Ther 2013; 93: 493-501

[1187] Thieme D, Rolf B, Sachs $\mathrm{H}$ et al. Correlation of inter-individual variations of amitriptyline metabolism examined in hairs with CYP2C19 and CYP2D6 polymorphisms. Int J Legal Med 2008; 122: 149-155

[1188] Tianmei S, Knadler MP, Lim MT et al. Pharmacokinetics and tolerability of duloxetine following oral administration to healthy Chinese subjects. Clin Pharmacokinet 2007; 46: 767-775

[1189] Timmer C], Sitsen JM, Delbressine LP. Clinical pharmacokinetics of mirtazapine. Clin Pharmacokinet 2000; 38: 461-474

[1190] Tiseo P], Rogers SL, Friedhoff LT. Pharmacokinetic and pharmacodynamic profile of donepezil $\mathrm{HCl}$ following evening administration. $\mathrm{Br}$ J Clin Pharmacol 1998; 46: (Suppl 1): 13-18

[1191] Titier K, Canal M, Deridet E et al. Determination of myocardium to plasma concentration ratios of five antipsychotic drugs: Comparison with their ability to induce arrhythmia and sudden death in clinical practice. Toxicol Appl Pharmacol 2004; 199: 52-60

[1192] Toennes SW, Maurer HH. Microsoft Excel in pharmacokinetics - an easy way to solve kinetic problems in clinical toxicology, legal medicine or doping control. In: Sachs H, Bernhard W, Jeger A. (eds.) Proceedings of the 34th International TIAFT Meeting, Interlaken. 11-15 August 1996. Leipzig: Molina; 1997: 201-204

[1193] Tokairin T, Fukasawa T, Yasui-Furukori N et al. Inhibition of the metabolism of brotizolam by erythromycin in humans: In vivo evidence for the involvement of CYP3A4 in brotizolam metabolism. $\mathrm{Br}$ J Clin Pharmacol 2005; 60: 172-175

[1194] Tokunaga H, Kudo K, Imamura T et al. Plasma concentrations of antipsychotic drugs in psychiatric inpatients. Nihon Hoigaku Zasshi 1997; 51: 417-422

[1195] Tolbert D, Bekersky I, Chu HM et al. An integrative population pharmacokinetics approach to the characterization of the effect of hepatic impairment on clobazam pharmacokinetics. J Clin Pharmacol 2016; 56: 213-222

[1196] Tomita T, Yasui-Furukori N, Nakagami T et al. Therapeutic reference range for plasma concentrations of paroxetine in patients with major depressive disorders. Ther Drug Monit 2014; 36: 480-485

[1197] Tompson DJ, Crean CS. Clinical pharmacokinetics of retigabine/ ezogabine. Curr Clin Pharmacol 2013; 8: 319-331

[1198] Tompson DJ, Vearer D. Steady-state pharmacokinetic properties of a 24-hour prolonged-release formulation of ropinirole: Results of two randomized studies in patients with Parkinson's disease. Clin Ther 2007; 29: 2654-2666

[1199] Tomson T. Landmark C], Battino D. Antiepileptic drug treatment in pregnancy: Changes in drug disposition and their clinical implications. Epilepsia 2013; 54: 405-414

[1200] Topinkova E, Baeyens JP, Michel JP et al. Evidence-based strategies for the optimization of pharmacotherapy in older people. Drugs Aging 2012; 29: 477-494

[1201] Topiwala A, Fazel S. The pharmacological management of violence in schizophrenia: a structured review. Expert Rev Neurother 2011; 11: 53-63

[1202] Tornio A, Neuvonen PJ, Backman JT. The CYP2C8 inhibitor gemfibrozil does not increase the plasma concentrations of zopiclone. Eur J Clin Pharmacol 2006; 62: 645-651

[1203] Totah RA, Sheffels P, Roberts T et al. Role of CYP2B6 in stereoselective human methadone metabolism. Anesthesiology 2008; 108: 363-374

[1204] Touw DJ, Neef C, Thomson AH et al. Cost-effectiveness of therapeutic drug monitoring: A systematic review. Ther Drug Monit 2005; $27: 10-17$
[1205] Traugott KA, Maxwell PR, Green K et al. Effects of therapeutic drug monitoring criteria in a computerized prescriber-order-entry system on the appropriateness of vancomycin level orders. Am J Health Syst Pharm 2011; 68: 347-352

[1206] Trivedi MH, Rush AJ, Gaynes BN et al. Maximizing the adequacy of medication treatment in controlled trials and clinical practice: $\operatorname{STAR}\left({ }^{*}\right)$ D measurement-based care. Neuropsychopharmacology 2007; 32: 2479-2489

[1207] Tsai MH, Lin KM, Hsiao MC et al. Genetic polymorphisms of cytochrome P450 enzymes influence metabolism of the antidepressant escitalopram and treatment response. Pharmacogenomics 2010; 11: 537-546

[1208] Tuerck D, Appel-Dingemanse S, Maboudian M et al. Dose-proportional pharmacokinetics of d-threo-methylphenidate after a repeated-action release dosage form. J Clin Pharmacol 2007; 47: 64-69

[1209] Tuerck D, Wang Y, Maboudian M et al. Similar bioavailability of dexmethylphenidate extended (bimodal) release, dexmethyl-phenidate immediate release and racemic methylphenidate extended (bimodal) release formulations in man. Int J. Clin Pharmacol Ther 2007; 45: 662-668

[1210] Turbott J, Norman TR, Burrows GD et al. Pharmacokinetics of nortriptyline in elderly volunteers. Commun Psychopharmacol 1980; 4: 225-231

[1211] Turpeinen M, Koivuviita N, Tolonen A et al. Effect of renal impairment on the pharmacokinetics of bupropion and its metabolites. Br J Clin Pharmacol 2007; 64: 165-173

[1212] Uchida H, Mamo DC, Mulsant BH et al. Increased antipsychotic sensitivity in elderly patients: Evidence and mechanisms. J Clin Psychiatry 2009; 70: 397-405

[1213] Uchida H, Suzuki T, Graff-Guerrero A et al. Therapeutic window for striatal dopamine $D(2 / 3)$ receptor occupancy in older patients with schizophrenia: A pilot PET study. Am J Geriatr Psychiatry 2014; 22: 1007-1016

[1214] Ufer M, von Stulpnagel C, Muhle H et al. Impact of ABCC2 genotype on antiepileptic drug response in Caucasian patients with childhood epilepsy. Pharmacogenet Genomics 2011; 21: 624-630

[1215] Uhr M, Grauer MT, Holsboer F. Differential enhancement of antidepressant penetration into the brain in mice with abcb1ab (mdr1 ab) P-glycoprotein gene disruption. Biol Psychiatry 2003; 54: 840-846

[1216] Uhr M, Steckler T, Yassouridis A et al. Penetration of amitriptyline, but not of fluoxetine, into brain is enhanced in mice with bloodbrain barrier deficiency due to mdr1a P-glycoprotein gene disruption. Neuropsychopharmacology 2000; 22: 380-387

[1217] Uhr M, Tontsch A, Namendorf C et al. Polymorphisms in the drug transporter gene $A B C B 1$ predict antidepressant treatment response in depression. Neuron 2008; 57: 203-209

[1218] Ujiie Y, Fukasawa T, Yasui-Furukori N et al. Rifampicin markedly decreases plasma concentration and hypnotic effect of brotizolam. Ther Drug Monit 2006; 28: 299-302

[1219] Ulrich S, Baumann B, Wolf R et al. Therapeutic drug monitoring of clozapine and relapse-a retrospective study of routine clinical data. Int J Clin Pharmacol Ther 2003; 41: 3-13

[1220] Ulrich S, Danos P, Baumann B et al. Serum concentration of chlormethiazole and therapeutic effect in acute alcohol withdrawal syndrome: An open clinical trial. Ther Drug Monit 2002; 24: 446-454

[1221] Ulrich S, Hiemke C, Laux G et al. Value and actuality of the prescription information for therapeutic drug monitoring of psychopharmaceuticals: A comparison with the medico-scientific evidence. Pharmacopsychiatry 2007; 40: 121-127 
[1222] Ulrich S, Lauter ]. Comprehensive survey of the relationship between serum concentration and therapeutic effect of amitriptyline in depression. Clin Pharmacokinet 2002; 41: 853-876

[1223] Ulrich S, Sandmann U, Genz A. Serum concentrations of haloperidol pyridinium metabolites and the relationship with tardive dyskinesia and parkinsonism: A cross-section study in psychiatric patients. Pharmacopsychiatry 2005; 38: 171-177

[1224] Ulrich S, Wurthmann C, Brosz M et al. The relationship between serum concentration and therapeutic effect of haloperidol in patients with acute schizophrenia. Clin Pharmacokinet 1998; 34 : 227-263

[1225] Unholzer S, Haen E. Retrospective analysis of therapeutic drug monitoring data for treatment of bipolar disorder with lamotrigine. Pharmacopsychiatry 2015; 48: 211-214

[1226] Unterecker S, Deckert J, Pfuhlmann B. No influence of body weight on serum levels of antidepressants. Ther Drug Monit 2011; 33: 730-734

[1227] Unterecker S, Pfuhlmann B, Kopf J et al. Increase of heart rate and QTc by amitriptyline, but not by venlafaxine, is correlated to serum concentration. J Clin Psychopharmacol 2015; 35: 460-463

[1228] Unterecker S, Riederer P, Proft F et al. Effects of gender and age on serum concentrations of antidepressants under naturalistic conditions.

J Neural Transm (Vienna) 2013; 120: 1237-1246

[1229] Valdes R Jr, Payne DA, Linder MW.(eds.) Laboratory medicine practice guidelines and recommendations for laboratory analysis and application of pharmacogenetics to clinical practice. Washington, DC: National Academy of Clinical Biochemistry; 2010

[1230] van den Anker JN. Developmental pharmacology. Dev Disabil Res Rev 2010; 16: 233-238

[1231] van der Lee M], Blenke AA, Rongen GA et al. Interaction study of the combined use of paroxetine and fosamprenavir-ritonavir in healthy subjects. Antimicrob Agents Chemother 2007; 51: 4098-4104

[1232] van der Weide J, Steijns LS, van Weelden MJ. The effect of smoking and cytochrome P450 CYP1A2 genetic polymorphism on clozapine clearance and dose requirement. Pharmacogenetics 2003; 13 : 169-172

[1233] van der Weide J, van Baalen-Benedek EH, Kootstra-Ros JE. Metabolic ratios of psychotropics as indication of cytochrome P450 2D6/2C19 genotype. Ther Drug Monit 2005; 27: 478-483

[1234] van Gerven JM, Uchida E, Uchida N et al. Pharmacodynamics and pharmacokinetics of a single oral dose of nitrazepam in healthy volunteers: an interethnic comparative study between Japanese and European volunteers. J Clin Pharmacol 1998; 38: 1129-1136

[1235] van Gorp F, Whyte IM, Isbister GK. Clinical and ECG effects of escitalopram overdose. Ann Emerg Med 2009; 54: 404-408

[1236] van Hasselt FM, Coehorst Y, Wilffert B et al. Influencing CYP enzymes to boost psychiatric treatment: A review on clinical evidence. Pharmacopsychiatry 2013; 46: 43-46

[1237] Van Putten T, Marder SR, Wirshing WC et al. Neuroleptic plasma levels. Schizophr Bull 1991; 17: 197-216

[1238] van Rongen A, Kervezee L, Brill M et al. Population Pharmacokinetic Model Characterizing 24-Hour Variation in the Pharmacokinetics of Oral and Intravenous Midazolam in Healthy Volunteers. CPT Pharmacometrics Syst Pharmacol 2015; 4: 454-464

[1239] van Steveninck AL, Wallnofer AE, Schoemaker RC et al. A study of the effects of long-term use on individual sensitivity to temazepam and lorazepam in a clinical population. Br J Clin Pharmacol 1997; 44: 267-275

[1240] Vandenberghe F, Guidi M, Choong E et al. Genetics-Based Population Pharmacokinetics and Pharmacodynamics of Risperidone in a Psychiatric Cohort. Clin Pharmacokinet 2015; 54: 1259-1272
[1241] VanderZwaag C, McGee M, McEvoy JP et al. Response of patients with treatment-refractory schizophrenia to clozapine within three serum level ranges. Am J Psychiatry 1996; 153: 1579-1584

[1242] Varsaldi F, Miglio G, Scordo MG et al. Impact of the CYP2D6 polymorphism on steady-state plasma concentrations and clinical outcome of donepezil in Alzheimer's disease patients. Eur J Clin Pharmacol 2006; 62: 721-726

[1243] Vasudev K, Das S, Goswami U et al. Pharmacokinetics of valproic acid in patients with bipolar disorder. J Psychopharmacol 2001; 15: 187-190

[1244] Vasudev K, Goswami U, Kohli K. Carbamazepine and valproate monotherapy: Feasibility, relative safety and efficacy, and therapeutic drug monitoring in manic disorder. Psychopharmacology (Berl) 2000; 150: 15-23

[1245] Veefkind AH, Haffmans PM, Hoencamp E. Venlafaxine serum levels and CYP2D6 genotype. Ther Drug Monit 2000; 22: 202-208

[1246] Velligan DI, Lam YW, Glahn DC et al. Defining and assessing adherence to oral antipsychotics: A review of the literature. Schizophr Bull 2006; 32: 724-742

[1247] Velligan DI, Weiden PJ, Sajatovic M et al. Assessment of adherence problems in patients with serious and persistent mental illness: recommendations from the Expert Consensus Guidelines. J Psychiatr Pract 2010; 16: 34-45

[1248] Venkatakrishnan K, Culm KE, Ehrenberg BL et al. Kinetics and dynamics of intravenous adinazolam, $\mathrm{N}$-desmethyl adinazolam, and alprazolam in healthy volunteers. J Clin Pharmacol 2005; 45: 529-537

[1249] Venkatakrishnan K, von Moltke LL, Greenblatt D]. Nortriptyline E-10-hydroxylation in vitro is mediated by human CYP2D6 (high affinity) and CYP3A4 (low affinity): Implications for interactions with enzyme-inducing drugs. J Clin Pharmacol 1999; 39: 567-577

[1250] Vermeir M, Naessens I, Remmerie B et al. Absorption, metabolism, and excretion of paliperidone, a new monoaminergic antagonist, in humans. Drug Metab Dispos 2008; 36: 769-779

[1251] Vernaleken I, Fellows C, Janouschek $\mathrm{H}$ et al. Striatal and extrastriatal D2/D3-receptor-binding properties of ziprasidone: a positron emission tomography study with [18F]Fallypride and [11C] raclopride (D2/D3-receptor occupancy of ziprasidone). J Clin Psychopharmacol 2008; 28: 608-617

[1252] Vernaleken I, Janouschek H, Raptis M et al. Dopamine D2/3 receptor occupancy by quetiapine in striatal and extrastriatal areas. Int J Neuropsychopharmacol 2010; 13: 951-960

[1253] Vernaleken I, Siessmeier T, Buchholz HG et al. High striatal occupancy of D2-like dopamine receptors by amisulpride in the brain of patients with schizophrenia. Int J Neuropsychopharmacol 2004; 7: 421-430

[1254] Verster JC, Roth T. Blood drug concentrations of benzodiazepines correlate poorly with actual driving impairment. Sleep Med Rev 2013; 17: 153-159

[1255] Verthein U, Ullmann R, Lachmann A et al. The effects of racemic $\mathrm{D}, \mathrm{L}$-methadone and L-methadone in substituted patients-a randomized controlled study. Drug Alcohol Depend 2005; 80: 267-271

[1256] Ververs FF, Voorbij HA, Zwarts P et al. Effect of cytochrome P450 2D6 genotype on maternal paroxetine plasma concentrations during pregnancy. Clin Pharmacokinet 2009; 48: 677-683

[1257] Veselinovic T, Paulzen M, Gründer G. Cariprazine, a new, orally active dopamine D2/3 receptor partial agonist for the treatment of schizophrenia, bipolar mania and depression. Expert Rev Neurother 2013; 13: 1141-1159

[1258] Vevelstad M, Pettersen S, Tallaksen C et al. O-demethylation of codeine to morphine inhibited by low-dose levomepromazine. Eur ] Clin Pharmacol 2009; 65: 795-801 
[1259] Vezmar S, Miljkovic B, Vucicevic K et al. Pharmacokinetics and efficacy of fluvoxamine and amitriptyline in depression. J Pharmacol Sci 2009; 110: 98-104

[1260] Viala A, Ba B, Durand A et al. Comparative study of the pharmacokinetics of zuclopenthixol decanoate and fluphenazine decanoate. Psychopharmacology (Berl) 1988; 94: 293-297

[1261] Vickers S, Stuart EK, Hucker HB. Further studies on the metabolism of carbidopa, (minus)-L-alpha-hydrazino-3,4-dihydroxy-alphamethylbenzenepropanoic acid monohydrate, in the human, Rhesus monkey, dog, and rat. J Med Chem 1975; 18: 134-138

[1262] Videla S, Cebrecos J, Lahjou M et al. Pharmacokinetic dose proportionality between two strengths (12.5 $\mathrm{mg}$ and $25 \mathrm{mg}$ ) of doxylamine hydrogen succinate film-coated tablets in fasting state: A single-dose, randomized, two-period crossover study in healthy volunteers. Drugs R D 2013; 13: 129-135

[1263] Viola MS, Bercellini MA, Saidon P et al. [Pharmacokinetic variability of oxcarbazepine in epileptic patients]. Medicina (B Aires) 2000; 60: 914-918

[1264] Vogel F, Gansmüller R, Leiblein T et al. The use of ziprasidone in clinical practice: Analysis of pharmacokinetic and pharmacodynamic aspects from data of a drug monitoring survey. Eur Psychiatry 2009; 24: 143-148

[1265] Voineskos AN, Wilson AA, Boovariwala A et al. Serotonin transporter occupancy of high-dose selective serotonin reuptake inhibitors during major depressive disorder measured with [11C]DASB positron emission tomography. Psychopharmacology (Berl) 2007; 193: 539-545

[1266] von Hentig N, Lotsch J. Cytochrome P450 3A inhibition by atazanavir and ritonavir, but not demography or drug formulation, influences saquinavir population pharmacokinetics in human immunodeficiency virus type 1-infected adults. Antimicrob Agents Chemother 2009; 53: 3524-3527

[1267] von Knorring L, Akerblad AC, Bengtsson F et al. Cost of depression: effect of adherence and treatment response. Eur Psychiatry 2006; 21: $349-354$

[1268] von Moltke LL, Greenblatt DJ, Giancarlo GM et al. Escitalopram (S-citalopram) and its metabolites in vitro: Cytochromes mediating biotransformation, inhibitory effects, and comparison to R-citalopram. Drug Metab Dispos 2001; 29: 1102-1109

[1269] Von Moltke LL, Greenblatt DJ, Granda BW et al. Zolpidem metabolism in vitro: Responsible cytochromes, chemical inhibitors, and in vivo correlations. Br J Clin Pharmacol 1999; 48: 89-97

[1270] von Moltke LL, Greenblatt DJ, Grassi JM et al. Protease inhibitors as inhibitors of human cytochromes P450: High risk associated with ritonavir. J Clin Pharmacol 1998; 38: 106-111

[1271] Vormfelde SV, Bitsch A, Meineke I et al. Non-response to maprotiline caused by ultra-rapid metabolism that is different from CYP2D6? Eur J Clin Pharmacol 1997; 52: 387-390

[1272] Vuille F, Amey M, Baumann P. Use of plasma level monitoring of antidepressants in clinical practice. Towards an analysis of clinical utility. Pharmacopsychiatry 1991; 24: 190-195

[1273] Waade RB, Christensen H, Rudberg I et al. Influence of comedication on serum concentrations of aripiprazole and dehydroaripiprazole. Ther Drug Monit 2009; 31: 233-238

[1274] Waldschmitt C, Vogel F, Pfuhlmann B et al. Duloxetine serum concentrations and clinical effects. Data from a therapeutic drug monitoring (TDM) survey. Pharmacopsychiatry 2009; 42: 189-193

[1275] Wan J, Xia H, He N et al. The elimination of diazepam in Chinese subjects is dependent on the mephenytoin oxidation phenotype. $\mathrm{Br}$ J Clin Pharmacol 1996; 42: 471-474

[1276] Wang JH, Liu ZQ, Wang W et al. Pharmacokinetics of sertraline in relation to genetic polymorphism of CYP2C19. Clin Pharmacol Ther 2001; 70: 42-47
[1277] Wang JS, Zhu HJ, Markowitz JS et al. Evaluation of antipsychotic drugs as inhibitors of multidrug resistance transporter P-glycoprotein. Psychopharmacology (Berl) 2006; 187: 415-423

[1278] Wang JS, Zhu HJ, Markowitz JS et al. Antipsychotic drugs inhibit the function of breast cancer resistance protein. Basic Clin Pharmacol Toxicol 2008; 103: 336-341

[1279] Wang P, Yin T, Ma HY et al. First analysis of the association between CYP3A4/5, ABCB1 genetic polymorphisms and oxcarbazepine metabolism and transport in chinese epileptic patients with oxcarbazepine monotherapy and bitherapy. J Pharm Pharm Sci 2015; 18: 256-265

[1280] Wang P, Yin T, Ma HY et al. Effects of CYP3A4/5 and ABCB1 genetic polymorphisms on carbamazepine metabolism and transport in Chinese patients with epilepsy treated with carbamazepine in monotherapy and bitherapy. Epilepsy Res 2015; 117: 52-57

[1281] Wang Q, Zhao L, Liang M et al. Effects of UGT2B7 genetic polymorphisms on serum concentrations of valproic acid in chinese children with epilepsy comedicated with lamotrigine. Ther Drug Monit 2016; 38: 343-349

[1282] Wang Y, Zhang H, Meng L et al. Influence of CYP2C19 on the relationship between pharmacokinetics and intragastric $\mathrm{pH}$ of omeprazole administered by successive intravenous infusions in Chinese healthy volunteers. Eur J Clin Pharmacol 2010; 66: 563-569

[1283] Ward ME, Musa MN, Bailey L. Clinical pharmacokinetics of lithium. ] Clin Pharmacol 1994; 34: 280-285

[1284] Wattmo C, Jedenius E, Blennow K et al. Dose and plasma concentration of galantamine in Alzheimer's disease - clinical application. Alzheimers Res Ther 2013; 5: 2

[1285] Weber ], McCormack PL, Asenapine CNS. Drugs 2009; 23: 781-792

[1286] Weiden PJ, Kozma C, Grogg A et al. Partial compliance and risk of rehospitalization among California Medicaid patients with schizophrenia. Psychiatr Serv 2004; 55: 886-891

[1287] Weigmann H, Bierbrauer ], Härtter S et al. Automated determination of clozapine and major metabolites in serum and urine. Ther Drug Monit 1997; 19: 480-488

[1288] Weigmann H, Härtter S, Hiemke C. Automated determination of clomipramine and its major metabolites in human and rat serum by high-performance liquid chromatography with on-line columnswitching. J Chromatogr B Biomed Sci Appl 1998; 710: 227-233

[1289] Weigmann H, Härtter S, Maehrlein S et al. Simultaneous determination of olanzapine, clozapine and demethylated metabolites in serum by on-line column-switching high-performance liquid chromatography. J Chromatogr B Biomed Sci Appl 2001; 759: 63-71

[1290] Weintraub D, Buchsbaum R, Spencer H et al. Tolerability of Levetiracetam in 516 patients: analysis by dose and serum concentration. Epilepsia 2004; 45: (Suppl 3): 68

[1291] Weiss RD, Griffin ML, Potter JS et al. Who benefits from additional drug counseling among prescription opioid-dependent patients receiving buprenorphine-naloxone and standard medical management? Drug Alcohol Depend 2014; 140: 118-122

[1292] Weiss U, Marksteiner ], Kemmler G et al. Effects of age and sex on olanzapine plasma concentrations. J Clin Psychopharmacol 2005; 25: $570-574$

[1293] Wen B, Ma L, Zhu M. Bioactivation of the tricyclic antidepressant amitriptyline and its metabolite nortriptyline to arene oxide intermediates in human liver microsomes and recombinant P450s. Chem Biol Interact 2008; 173: 59-67

[1294] Wen B, Zhou M. Metabolic activation of the phenothiazine antipsychotics chlorpromazine and thioridazine to electrophilic iminoquinone species in human liver microsomes and recombinant P450s. Chem Biol Interact 2009; 181: 220-226 
[1295] Wenzel-Seifert K, Brandl R, Hiemke C et al. Influence of concomitant medications on the total clearance and the risk for supra-therapeutic plasma concentrations of Citalopram. A population-based cohort study. Pharmacopsychiatry 2014; 47: 239-244

[1296] Wessels AM, Bies RR, Pollock BG et al. Population pharmacokinetic modeling of ziprasidone in patients with schizophrenia from the CATIE study. J Clin Pharmacol 2011; 51: 1587-1591

[1297] White N, Litovitz T, Clancy C. Suicidal antidepressant overdoses: a comparative analysis by antidepressant type. J Med Toxicol 2008; 4: $238-250$

[1298] Whitney Z, Boyda HN, Procyshyn RM et al. Therapeutic drug levels of second generation antipsychotics in youth: a systematic review. J Child Adolesc Psychopharmacol 2015; 25: 234-245

[1299] Wienkers LC, Allievi C, Hauer M] et al. Cytochrome P-450-mediated metabolism of the individual enantiomers of the antidepressant agent reboxetine in human liver microsomes. Drug Metab Dispos 1999; 27: 1334-1340

[1300] Wiesel FA, Alfredsson G, Ehrnebo M et al. The pharmacokinetics of intravenous and oral sulpiride in healthy human subjects. Eur J Clin Pharmacol 1980; 17: 385-391

[1301] Wigal SB, Gupta S, Heverin E et al. Pharmacokinetics and therapeutic effect of OROS methylphenidate under different breakfast conditions in children with attention-deficit/hyperactivity disorder. J Child Adolesc Psychopharmacol 2011; 21: 255-263

[1302] Wilczynski S, Koprowski R, Blonska-Fajfrowska B. Directional reflectance analysis for identifying counterfeit drugs: Preliminary study.

J Pharm Biomed Anal 2016; 124: 341-346

[1303] Wilhelm A], den Burger JC, Swart EL. Therapeutic drug monitoring by dried blood spot: Progress to date and future directions. Clin Pharmacokinet 2014; 53: 961-973

[1304] Wille SM, Cooreman SG, Neels HM et al. Relevant issues in the monitoring and the toxicology of antidepressants. Crit Rev Clin Lab Sci 2008; 45: 25-89

[1305] Willmore LJ, Abelson MB, Ben-Menachem E et al. Vigabatrin: 2008 update. Epilepsia 2009; 50: 163-173

[1306] Wilson JF. Survey of reference ranges and clinical measurements for psychoactive drugs in serum. Ther Drug Monit 2003; 25: 243-247

[1307] Wilting I, Heerdink ER, Mersch PP et al. Association between lithium serum level, mood state, and patient-reported adverse drug reactions during long-term lithium treatment: A naturalistic follow-up study. Bipolar Disord 2009; 11: 434-440

[1308] Wimmer S, Neubert A, Rascher W. The Safety of Drug Therapy in Children. Dtsch Arztebl Int 2015; 112: 781-787

[1309] Winblad B, Grossberg G, Frolich L et al. IDEAL: A 6-month, double-blind, placebo-controlled study of the first skin patch for Alzheimer disease. Neurology 2007; 69: S14-S22

[1310] Wincor MZ, Munjack DJ, Palmer R. Alprazolam levels and response in panic disorder: Preliminary results. J Clin Psychopharmacol 1991; 11: 48-51

[1311] Windhager E, Kim SW, Saria A et al. Perinatal use of aripiprazole: plasma levels, placental transfer, and child outcome in 3 new cases. J Clin Psychopharmacol 2014; 34: 637-641

[1312] Winter HR, Earley WR, Hamer-Maansson JE et al. Steady-state pharmacokinetic, safety, and tolerability profiles of quetiapine, norquetiapine, and other quetiapine metabolites in pediatric and adult patients with psychotic disorders. J Child Adolesc Psychopharmacol 2008; 18: 81-98

[1313] Wire MB, Shelton M], Studenberg S. Fosamprenavir : Clinical pharmacokinetics and drug interactions of the amprenavir prodrug. Clin Pharmacokinet 2006; 45: 137-168

[1314] Wohkittel C, Gerlach M, Taurines R et al. Relationship between clozapine dose, serum concentration, and clinical outcome in children and adolescents in clinical practice. J Neural Transm (Vienna) 2016; 123: 1021-1031

[1315] Wojcikowski J, Basinska A, Daniel WA. The cytochrome P450-catalyzed metabolism of levomepromazine: A phenothiazine neuroleptic with a wide spectrum of clinical application. Biochem Pharmacol 2014; 90: 188-195

[1316] Wojcikowski ], Boksa J, Daniel WA. Main contribution of the cytochrome P450 isoenzyme 1A2 (CYP1A2) to N-demethylation and 5-sulfoxidation of the phenothiazine neuroleptic chlorpromazine in human liver-A comparison with other phenothiazines. Biochem Pharmacol 2010; 80: 1252-1259

[1317] Wojcikowski J, Daniel WA. Perazine at therapeutic drug concentrations inhibits human cytochrome P450 isoenzyme 1A2 (CYP1A2) and caffeine metabolism-an in vitro study. Pharmacol Rep 2009; 61: 851-858

[1318] Wojcikowski ], Pichard-Garcia L, Maurel P et al. Contribution of human cytochrome $\mathrm{p}-450$ isoforms to the metabolism of the simplest phenothiazine neuroleptic promazine. $\mathrm{Br}$ ] Pharmacol 2003; 138: 1465-1474

[1319] Wolff K, Hay AW, Raistrick D et al. Steady-state pharmacokinetics of methadone in opioid addicts. Eur J Clin Pharmacol 1993; 44: 189-194

[1320] Wolking S, Schaeffeler E, Lerche H et al. Impact of Genetic Polymorphisms of ABCB1 (MDR1, P-Glycoprotein) on Drug Disposition and Potential Clinical Implications: Update of the Literature. Clin Pharmacokinet 2015; 54: 709-735

[1321] Wong SL, Granneman GR. Modeling of sertindole pharmacokinetic disposition in healthy volunteers in short term dose-escalation studies. J Pharm Sci 1998; 87: 1629-1631

[1322] Wong SL, Menacherry S, Mulford D et al. Pharmacokinetics of sertindole and dehydrosertindole in volunteers with normal or impaired renal function. Eur J Clin Pharmacol 1997; 52: 223-227

[1323] Wong YN, King SP, Simcoe D et al. Open-label, single-dose pharmacokinetic study of modafinil tablets: Influence of age and gender in normal subjects. J Clin Pharmacol 1999; 39: 281-288

[1324] Wong YN, Simcoe D, Hartman LN et al. A double-blind, placebocontrolled, ascending-dose evaluation of the pharmacokinetics and tolerability of modafinil tablets in healthy male volunteers. J Clin Pharmacol 1999; 39: 30-40

[1325] World Haelth Organization. Adherence to long term therapy: evidence for action. 2003; http://www.who.int/hiv/pub/prev_care/ Ittherapies/en/ [assessed May 13 2016]

[1326] Wright CE, Sisson TL, Ichhpurani AK et al. Steady-state pharmacokinetic properties of pramipexole in healthy volunteers. J Clin Pharmacol 1997; 37: 520-525

[1327] Wu MJ, Ing TS, Soung LS et al. Amantadine hydrochloride pharmacokinetics in patients with impaired renal function. Clin Nephrol 1982; 17: 19-23

[1328] Wu MK, Chung W, Wu CK et al. The severe complication of Stevens-Johnson syndrome induced by long-term clozapine treatment in a male schizophrenia patient: A case report. Neuropsychiatr Dis Treat 2015; 11: 1039-1041

[1329] Wynalda MA, Wienkers LC. Assessment of potential interactions between dopamine receptor agonists and various human cytochrome P450 enzymes using a simple in vitro inhibition screen. Drug Metab Dispos 1997; 25: 1211-1214

[1330] Xiang Q, Zhao X, Zhou Y et al. Effect of CYP2D6, CYP3A5, and MDR genetic polymorphisms on the pharmacokinetics of risperidone and its active moiety. J Clin Pharmacol 2010; 50: 659-666

[1331] Xu M, Zhou Y, Ni Y et al. Tolerability and pharmacokinetic comparison of oral, intramuscular, and intravenous administration of levosulpiride after single and multiple dosing in healthy chinese volunteers. Clin Ther 2015; 37: 2458-2467 
[1332] Xu P, Li HD, Zhang BK et al. Pharmacokinetics and tolerability of modafinil tablets in Chinese subjects. J Clin Pharm Ther 2008; 33 : 429-437

[1333] Xu RA, Gu EM, Zhou Q et al. Effects of 22 novel CYP2D6 variants found in Chinese population on the metabolism of dapoxetine. Drug Des Devel Ther 2016; 10: 687-696

[1334] Yao C, Raoufinia A, Gold M et al. Steady-state pharmacokinetics of galantamine are not affected by addition of memantine in healthy subjects. J Clin Pharmacol 2005; 45: 519-528

[1335] Yasui-Furukori N, Nakagami T, Kaneda A et al. Inverse correlation between clinical response to paroxetine and plasma drug concentration in patients with major depressive disorders. Hum Psychopharmacol 2011; 26: 602-608

[1336] Yasui-Furukori N, Saito M, Nakagami T et al. Clinical response to risperidone in relation to plasma drug concentrations in acutely exacerbated schizophrenic patients. J Psychopharmacol 2010; 24: 987-994

[1337] Yasui-Furukori N, Saito M, Nakagami T et al. Association between multidrug resistance 1 (MDR1) gene polymorphisms and therapeutic response to bromperidol in schizophrenic patients: A preliminary study. Prog Neuropsychopharmacol Biol Psychiatry 2006; 30: 286-291

[1338] Yeung PK, Hubbard JW, Korchinski ED et al. Pharmacokinetics of chlorpromazine and key metabolites. Eur J Clin Pharmacol 1993; 45: 563-569

[1339] Yin OQ, Wing YK, Cheung Y et al. Phenotype-genotype relationship and clinical effects of citalopram in Chinese patients. J Clin Psychopharmacol 2006; 26: 367-372

[1340] Yonkers KA, Kando JC, Cole JO et al. Gender differences in pharmacokinetics and pharmacodynamics of psychotropic medication. Am J Psychiatry 1992; 149: 587-595

[1341] Yu DK, Dimmitt DC, Lanman RC et al. Pharmacokinetics of dothiepin in humans: A single dose dose-proportionality study. J Pharm Sci 1986; 75: 582-585

[1342] Yu Y, Zhang Q, Xu W et al. Population pharmacokinetic modeling of oxcarbazepine active metabolite in Chinese patients with epilepsy. Eur J Drug Metab Pharmacokinet 2016; 41: 345-351

[1343] Zand R, Nelson SD, Slattery JT et al. Inhibition and induction of cytochrome P4502E1-catalyzed oxidation by isoniazid in humans. Clin Pharmacol Ther 1993; 54: 142-149

[1344] Zanger UM, Schwab M. Cytochrome P450 enzymes in drug metabolism: Regulation of gene expression, enzyme activities, and impact of genetic variation. Pharmacol Ther 2013; 138: 103-141
[1345] Zernig G, Hiemke C, Havemann-Reinecke U et al. Empfehlungen für die gutachterliche Bewertung von Medikamentenspiegeln in der Psychiatrie im gerichtsanhängigen Schadensfall. Psychopharmakotherapie 2009; 16: 57-64

[1346] Zernig G, Lechner T, Kramer-Reinstadler $\mathrm{K}$ et al. What the clinician still has to be reminded of. Ther Drug Monit 2004; 26: 582

[1347] Zernig G, Ng K, Hiemke C et al. Therapeutic drug monitoring-based clozapine dosing recommendations. Ther Drug Monit 2007; 29: 130-131

[1348] Zhao Q, lyer GR, Verhaeghe T et al. Pharmacokinetics and safety of galantamine in subjects with hepatic impairment and healthy volunteers. J Clin Pharmacol 2002; 42: 428-436

[1349] Zhaoxu L, Jingcheng T, Jinnan Z. Rifabutin autoinduction is caused by involvement of cytochrome P450 and cholinesterase. Pharmazie 2008; 63: 156-159

[1350] Zhou D, Bui KH, Li ] et al. Population pharmacokinetic modeling of quetiapine after administration of seroquel and seroquel XR formulations to Western and Chinese patients with schizophrenia, schizoaffective disorder, or bipolar disorder. J Clin Pharmacol 2015; 55: $1248-1255$

[1351] Zhou SF. Polymorphism of human cytochrome P450 2D6 and its clinical significance: Part I. Clin Pharmacokinet 2009; 48: 689-723

[1352] Zhou SF. Polymorphism of human cytochrome P450 2D6 and its clinical significance: part II. Clin Pharmacokinet 2009; 48: 761-804

[1353] Zhou SF, Liu JP, Chowbay B. Polymorphism of human cytochrome P450 enzymes and its clinical impact. Drug Metab Rev 2009; 41: 89-295

[1354] Zhu HJ, Wang JS, Donovan JL et al. Interactions of attention-deficit/ hyperactivity disorder therapeutic agents with the efflux transporter P-glycoprotein. Eur J Pharmacol 2008; 578: 148-158

[1355] Zingmark PH, Ekblom M, Odergren T et al. Population pharmacokinetics of clomethiazole and its effect on the natural course of sedation in acute stroke patients. Br J Clin Pharmacol 2003; 56: 173-183

[1356] Zullig LL, Peterson ED, Bosworth HB. Ingredients of successful interventions to improve medication adherence. JAMA 2013; 310: 2611-2612

[1357] Zullino DF, Delessert D, Eap CB et al. Tobacco and cannabis smoking cessation can lead to intoxication with clozapine or olanzapine. Int Clin Psychopharmacol 2002; 17: 141-143

[1358] Zweben A, Pettinati HM, Weiss RD et al. Relationship between medication adherence and treatment outcomes: the COMBINE study. Alcohol Clin Exp Res 2008; 32: 1661-1669

\section{Erratum}

C. Hiemke, N. Bergemann, H. W. Clement et al. Consensus Guidelines for Therapeutic Drug Monitoring in Neuropsychopharmacology: Update 2017

Pharmacopsychiatry 2017

DOI: $10.1055 / \mathrm{s}-0043-116492$

The online-version of this contribution has been corrected and uploaded on January 31, 2018. 